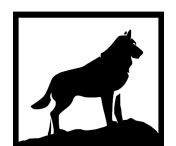

Michigan Technological

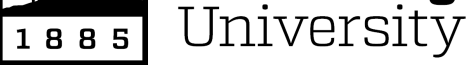

Michigan Technological University Digital Commons @ Michigan Tech

\title{
THE STUDY OF CHEMICAL INDUCED POLYOLEFIN-BASED ION EXCHANGE MEMBRANE FOR ELECTRODIALYSIS APPLICATION
}

Di Huang

Michigan Technological University, hdi@mtu.edu

Copyright 2020 Di Huang

\section{Recommended Citation}

Huang, Di, "THE STUDY OF CHEMICAL INDUCED POLYOLEFIN-BASED ION EXCHANGE MEMBRANE FOR ELECTRODIALYSIS APPLICATION", Open Access Dissertation, Michigan Technological University, 2020. https://doi.org/10.37099/mtu.dc.etdr/1103

Follow this and additional works at: https://digitalcommons.mtu.edu/etdr

Part of the Polymer and Organic Materials Commons 
THE STUDY OF CHEMICAL INDUCED POLYOLEFIN-BASED ION EXCHANGE MEMBRANE FOR ELECTRODIALYSIS APPLICATION

\author{
By \\ Di Huang
}

\begin{abstract}
A DISSERTATION
Submitted in partial fulfillment of the requirements for the degree of DOCTOR OF PHILOSOPHY

In Materials Science and Engineering
\end{abstract}

MICHIGAN TECHNOLOGICAL UNIVERSITY

2020

(C) 2020 Di Huang 
This dissertation has been approved in partial fulfillment of the requirements for the Degree of DOCTOR OF PHILOSOPHY in Materials Science and Engineering.

Department of Materials Science and Engineering

Dissertation Advisor: Jiann-Yang Hwang

Committee Member: Stephen A. Hackney

Committee Member: Bowen Li

Committee Member: Song-Lin Yang

Department Chair: Stephen L. Kampe 


\section{Table of Contents}

Preface

Acknowledgements ..............................................................................................

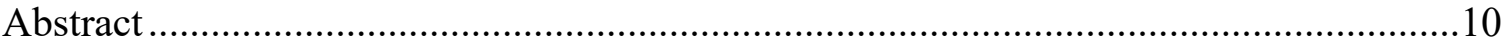

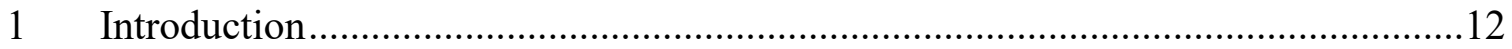

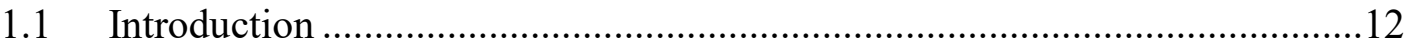

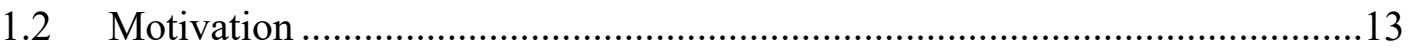

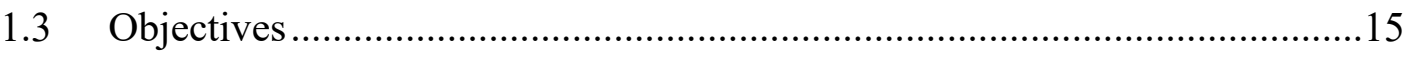

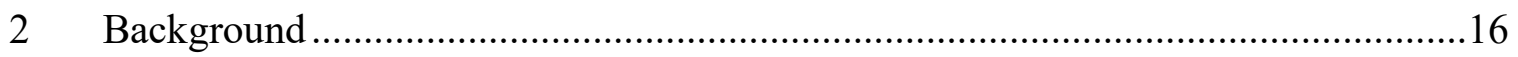

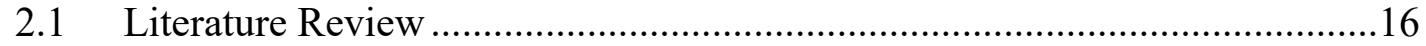

2.2 Inert polymer (backbone polymer) types of ion exchange membrane............18

2.2.1 Fluorine-containing hydrocarbons-based membranes .......................18

2.2.2 Nonfluorinated hydrocarbons based membranes................................19

2.2.2.1 Polyethylene-based ion exchange membrane .................20

2.2.2.2 Styrene-divinylbenzene-based ion exchange membranes 212.2.2.3 .......Polysulfone-based ion exchange membranes 22

2.3 Active monomers types of ion exchange membrane.....................................22

$2.4 \quad$ Fabrication methods of ion exchange membranes .........................................24

2.4.1 Radiation grafting polymerization method .......................................24

2.4.2 Plasma grafting polymerization method ..........................................25

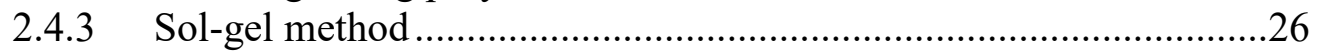

2.4.4 Monomers direct polymerization method......................................27

2.5 Ions conducting mechanisms in ion exchange membrane .............................27

2.5.1 Cation conduction mechanisms ...................................................2

2.5.2 Anion conduction mechanism.......................................................28

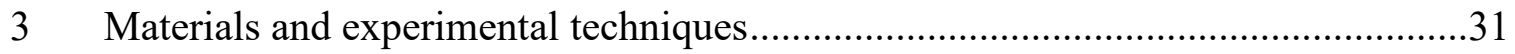

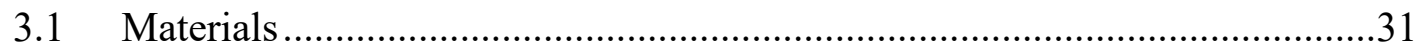

3.1.1 Materials for cation exchange membrane and anion exchange

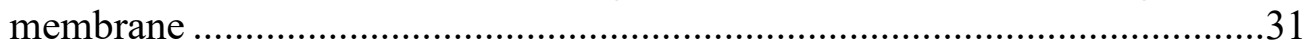

3.1.2 Fillers/ion exchange membrane composite.......................................... 31

3.1.2.1 Glass fiber/ion exchange membrane composite ..............31

3.1.2.2 Graphene oxide/ cation exchange membrane composite 31 


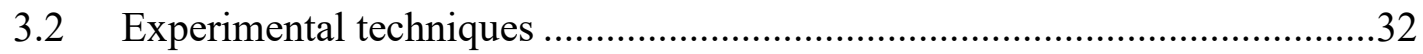

3.2.1 Cation exchange membrane preparation..............................................32

3.2.1.1 Synthesis of PE-graft-PS copolymer ...............................32

3.2.1.2 Sulfonation of PE-g-PS copolymer for cation exchange membrane preparation .......................................................................32

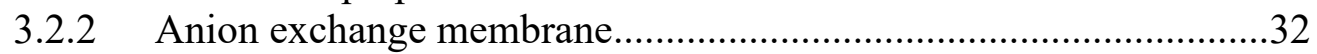

3.2.2.1 Synthesis of LDPE-graft-Poly (St-co-VBC) copolymer.32

3.2.2.2 Amination of LDPE-graft-Poly (St-co-VBC) for anion exchange membrane preparation ........................................................33

3.2.3 Glass fiber/ion exchange membrane composite preparation .............33

3.2.3.1 Surface treatment of glass fiber .......................................33

3.2.3.2 Synthesis and sulfonation of PE-graft-PS/GF membrane composite 34

3.2.4 Graphene oxide derivate/cation exchange membrane composite preparation.

Preparation of graphene oxide (GO)

3.2.4.2 Preparation of aminopropyl silane graphene oxide (MGO) 35

3.2.4.3 Preparation of sulfanilic acid modified MGO (S-MGO) 36

3.2.4.4 Preparation of phosphorous acid and sulfanilic acid modified MGO (PS-MGO) .............................................................

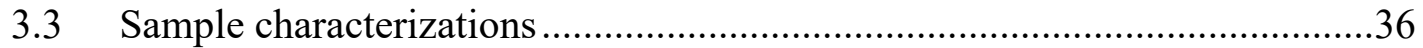

3.3.1 Fourier-transform infrared spectroscopy (FT-IR) analysis .................36

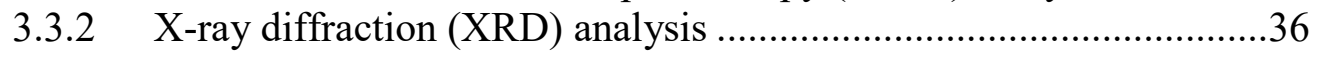

3.3.3 Morphology characterization ...............................................................

3.3.4 X-ray photoelectron spectrometer characterization ..............................37

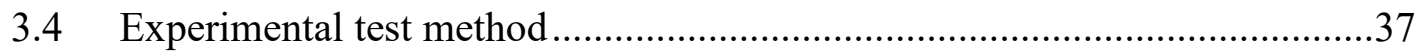

3.4.1 Mechanical properties.......................................................................

3.4.2 Ion exchange capacity (IEC) ............................................................

3.4.3 Water uptake and Swelling rate ......................................................39

3.4.4 Membrane conductivity and activation energy measurements .........40

3.4.5 Electrochemical analysis and electrodialysis cell evaluation ............42

4 Investigation of polyolefin-based cation exchange membrane..................................43

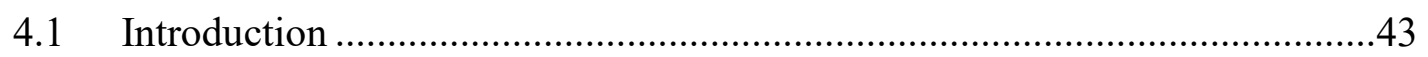

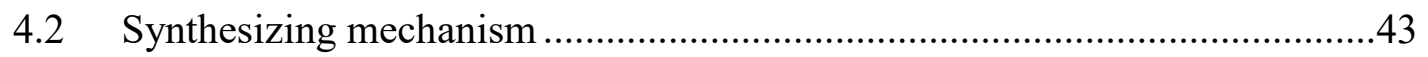

4.3 Experimental results and discussion............................................................45

4.3.1 The effect of inert polymer type on cation exchange membrane properties.

4.3.1.1 The effect of polyethylene structure on ion exchange

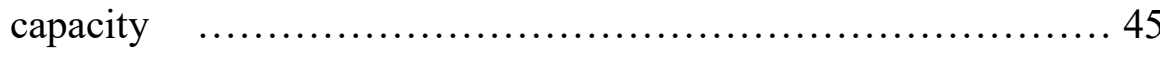


4.3.1.2 FT-IR spectrum analysis

4.3.1.3 The effect of other inert polymer types on membrane properties

4.3.1.3.1 Physical properties ................................ 53

4.3.1.3.2 Thermal expansion property .........................54

4.3.1.3.3 Morphology characterization .......................55

4.3.2 The effect of styrene addition on cation exchange membrane properties.....

4.3.2.1 The effect of styrene addition on ion exchange capacity 56

4.3.2.2 FT-IR spectrum analysis .61

4.3.3 The effect of the crosslinking degree on cation exchange membrane properties

4.3.3.1 The effect of the crosslinking degree on IEC 62

4.3.3.2 The effects of the crosslinking degree on burst strength 63

4.3.3.3 FTIR analysis 64

4.3.4 The effect of solfonaiton condition on cation exchange membrane properties

4.3.5 The effect of the initiator (BPO) concentration on cation exchange membrane properties .68

4.4 Summary .....

5 Investigation the polyolefin-based anion exchange membrane .70

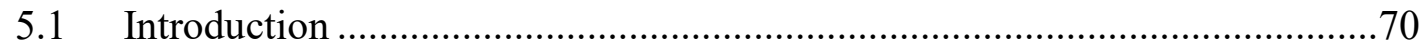

5.2 Comparation of synthesizing methods .73

5.2.1 Chloromethylation method to synthesize AEM (PE-g-PS based AEM) 73

5.2.2 4-Vinylbenzyl Chloride method to synthesize AEM (PE-g-PVBC based AEM).

5.2.3 4-Vinylbenzyl Chloride and styrene co-monomer method to synthesize AEM (PE-g-PS/PVBC based AEM). .75

5.3 Experimental results and discussion.............................................................

5.3.1 The effect of chloromethylation reaction condition on anion exchange membrane properties .75

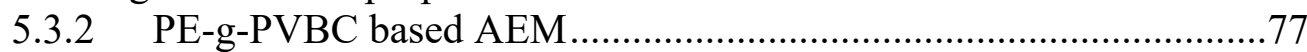

5.3.3 The effect of styrene on non-radiation grafting PE-g-PVBC AEM .81 5.3.3.1 Characterization of AEM by FTIR spectroscopy analysis

5.3.3.2 Characterization of AEM by SEM and TEM analysis....83

5.3.3.3 Ion exchange capacity (IEC)............................................85

5.3.3.4 Water uptake and membrane swelling ratio.....................86 
5.3.3.5 Comparation of working performance for AEM with and

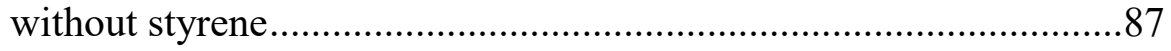

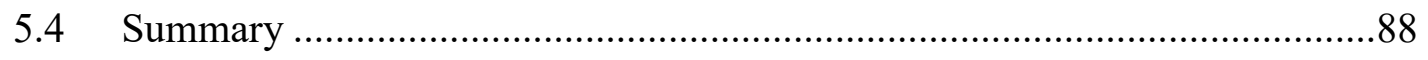

6 Studies on glass fiber reinforced poly(ethylene-grafted-styrene) based cation

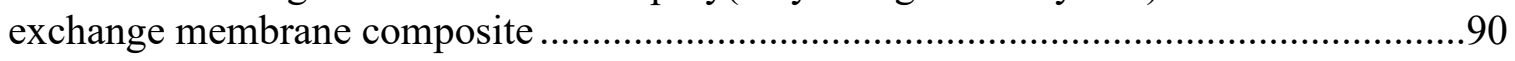

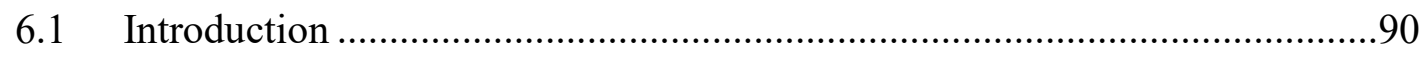

6.2 Experimental results and discussions ........................................................92

6.2.1 The effect of various coupling agents on mechanical properties of

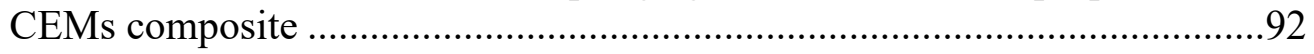

6.2.2 The effect of various coupling agents on dynamic mechanical property of IEMs composite ..........................................................................99

6.2.3 CEMs composite morphology analysis .............................................98

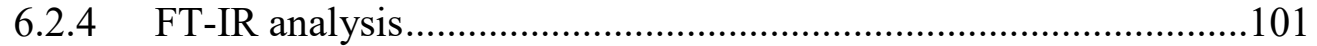

6.2.5 Effect of GF on Ion Exchange Capacity .........................................104

6.2.6 Effect of GF/CEMs composite on water uptake and swelling rate.105

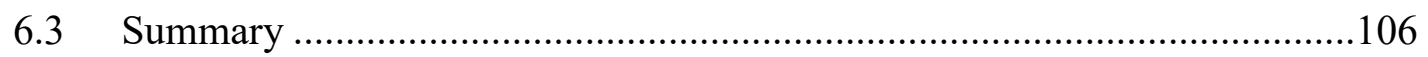

7 Dually acid-doped functionalized graphene oxide/sulfonated poly (ethylene-costyrene) cation exchange membrane composite ………………………………….......108

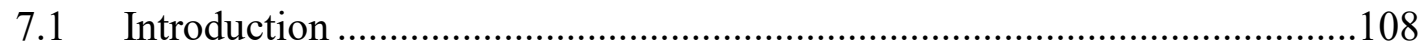

7.2 Products design and synthesis procedure ………….....................................

7.3 Experimental results and discussion........................................................111

7.3.1 Characterization of the functionalized graphene oxide and membrane composites

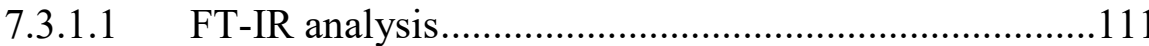

7.3.1.2 XRD analysis ..............................................................112

7.3.1.3 XPS characterization and analysis ..................................113

7.3.1.4 Morphology characterization and analysis ...................118

7.3.2 Mechanical properties of PS-MGO/CEM and S-MGO/CEM

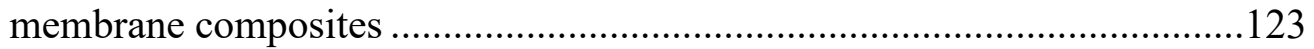

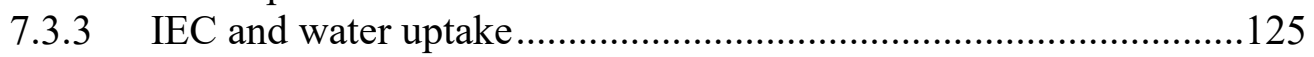

7.3.4 Electrochemical impedance spectroscopy result and analysis........127

7.3.4.1 7.3.4.1 Principle of Electrochemical impedance

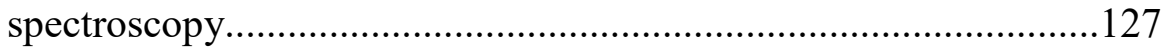

7.3.4.2 EIS measurement .....................................................129

7.3.4.3 EIS measure and analysis..............................................131

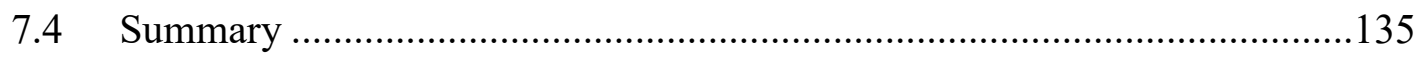


8 Conclusion and recommendations for future work ......................................136

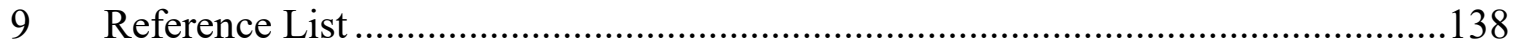

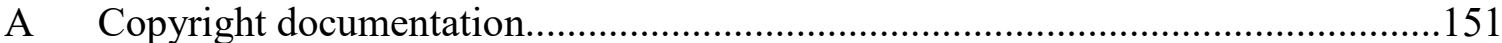




\section{Preface}

This dissertation includes three chapters from my publication manuscripts. The chapter 5 has been accepted for publication at Characterization of Minerals, Metals and Materials 2020 (Di Huang, Jiann-Yang Hwang, Zhichao Chen, "Study of Styrene Effect on Nonradiation Grafting on to Polyethylene-Based Anion Exchange Membrane", Characterization of Minerals, Metals, and Materials 2020, Springer, 2020, pp.701-710). The chapter 6 has been accepted for publication at Materials Journal (Di Huang, Chen, Zhichao, and Jiann-Yang Hwang." Studies on Glass Fiber Reinforced Poly(ethylenegrafted-styrene) based Cation Exchange Membrane Composite." Materials 2020, 13(24), 5597.) The chapter 7 are yet to be submitted to the Journal of Materials and Polymer International under reviewing.

In terms of contribution to this work, Dr.Zhichao Chen helped me grasp the copolymer synthesizing theory included in chapters 2 and 3 . He also gave me useful information about characterization and test for membrane material. Many valuable recommendations for researching directions and additional analyzing methods were also guided by Prof. Jiann-yang Hwang. Owen Mills and Pinaki assisted with transmission electron microscopy and field emission scanning electron microscopy analysis in chapters 5, 6 and 7. Paul Fraley offered me essential training and useful suggestions on dynamic mechanical analysis tests in chapters 4,5 and 7 .

Dr. Timothy Leftwich assisted with X-ray photoelectron spectroscopy survey scanning and high-resolution scanning included in chapter 7 . All other data in this study was collected and analyzed by the author. 


\section{Acknowledgements}

Most importantly, I would like to give my most sincere thanks to my advisor Prof. Jiannyang Hwang and my colleague Dr. Zhichao Chen for their many supports, guidance and instructions. I will not reach my researching goal without them.

This work would not have been possible without the financial, moral and technical support from the following agencies, faculties, staff and family and friends.

Ever Green Technological, Inc.

Futianbao Environmental Protection Technology, Ltd.

Prof. Stephen L. Kampe, Prof. Stephen Hackney, Prof. Jaroslaw Drelich, Dr. Pinaki, Dr. Dan Seguin, Dr. Edward Laitila, Dr. Timothy Leftwich and Dr. Malgorzata

Owen Mills, Paul Fraley, Rachel Griffin and Valentina O'kane

Dr. Yuzhe Zhang, Dr. Xin Yan, Changjun Lee

My family, Mom, Dad, my husband and our kids 


\section{Abstract}

High-performance ion exchange membranes with high ion exchange capacity (IEC), excellent mechanical properties, lower membrane resistance and superior ions conductivity were developed with chemical-induced polymerization in this work. Through a series of synthesizing experiments, structure characterization and properties testing for polyolefin-based cation exchange membrane (CEM) and anion exchange membrane (AEM), LDPE proved to be a optimized backbone material. The CEM with $57.5 \%$ styrene, $38.4 \%$ LDPE, 3\% crosslinking degree and $1 \%$ initiator addition yield the highest IEC value $(1.72 \mathrm{~mol} / \mathrm{g})$ and moderate burst strength. The $10 \%$ addition of styrene was found to enhance IEC of $57 \%$ to AEM. However, continually increase styrene leaded lower IEC due to the decreasing grafting degree of vinyl benzene chloride (VBC) on polyethylene.

The influence of fillers, such as surface modified glass fiber (GF) and functionalized graphene oxides $(\mathrm{GO})$, on thermal, mechanical and electrochemical properties of ion exchange membrane were investigated in this work by dynamic mechanical analysis, IEC and field emission scanning electron microscopes (FE-SEM), fourier-transform infrared spectroscopy (FT-IR) and electrochemical impedance spectroscopy. The addition of modified GF increases tensile strength, tensile modulus, storage modulus and interfacial adhesion of GF/CEM composite but degraded the strains. The composite with [3(Methacryloxy) propyl] trimethoxy silane (3-MPS) modified GF obtained superior mechanical properties and interfacial adhesion, whereas the modified effect of triethoxyvinylsilane (TES) was inconspicuous. The addition of unmodified GF even had negative effects on GF/CEM mechanical properties. The FE-SEM showed that the GF treated by 3-MPS and poly(propylene-graft-maleic anhydride) (PP-g-MA) have better compatibility with the CEM matrix than 1,6 bis and TES treated GF. The FT-IR verified the strengthening effects from modified GF were attributed to the formation of Si-O-Si and Si-O-C bonds. The additions of modified GF in CEM positively influence water uptake ability but negatively on IEC. This section provided a way of strengthening GF/CEM composite.

The CEM doped with functionalized graphene oxides was verified to be significantly improved in IEC (21\% higher) and membrane conductivity (326.7\% higher) compare to the pristine CEM. The results also suggested that the improved effects of dualfunctionalized $\mathrm{GO}$ on $\mathrm{CEM}$ properties are superior to the single functionalized $\mathrm{GO}$. The coexistence of $-\mathrm{PO}_{3} \mathrm{H},-\mathrm{SO}_{3} \mathrm{H}$ in $\mathrm{GO}$ resulted CEM possessed $7.8 \%$ higher IEC, $77.29 \%$ higher membrane conductivity and $43.56 \%$ lower activation energy than that with single functionalized GO. This work provides a new strategy on the design of high-performance IEM with excellent mechanical property, high IEC, high conductivity and low membrane resistance for application. 


\section{Introduction}

\subsection{Introduction}

The applications of membrane separation techniques for reverse osmosis, nanofiltration, ultrafiltration, microfiltration, pervaporation separation and electrodialysis process have been increasing rapidly [1]. Electrodialysis is one of the separation techniques in which counterions in electrolyte exchange with fixed ions attached on membrane under the electrostatic force. Consequently, the counterions transport through membranes and move towards opposite electrode by the electrical potential difference [2]. The widest applications of electrodialysis focused on concentration of electrolyte solutions or deionization of solutions [3-4]. This process has been widely used to produce drinking water from brackish water, treat industrial wastewater and recycle useful substance from effluents and salt production [18]. Ion exchange membranes (IEM), as one of the core components of electrodialysis, have been universally applied to seawater desalination as well as brackish water concentration, pharmaceutical purification, water softening processes and fuel cell separators etc. [5-8]. IEM are made of polymeric materials with charged groups attaching on them. Ions are selectively permeated through the membrane. The exchange between counter ions and fixed ions on IEM based on the Gibbs-Donnan effect. The Gibbs-Donnan effect can be described when there are two opposite charged substances near a semi-permeable membrane, some ions are unable to pass through the membrane and thus creates uneven electrical charges distribute across the membranes. The usual cause is the presence of a different charged ions attached on membrane surface that hinder equal ions permeation [9].

Typically, IEMs are composed of inert polymers such as polyethylene, polyvinylidene fluoride and polyvinylchloride, and reactive polymers such as polystyrene, polystyrene and polysulfone that can be functionalized with ionic groups [15]. The desired properties of IEMs are determined by the inert backbone polymers, reactive polymers and methods to synthesize IEC. The backbone polymers are related on the mechanical properties, chemical and thermal stability of IEM. Most inert polymer presented in IEMs are hydrophobic and lead to poor electrochemical properties such as low conductivity and selectivity of ions [16]. Therefore, the introduction of hydrophilic reactive monomers attached by acidic or basic ion groups is necessary. The reactive monomers types and compatibility with inert polymer greatly affect the ion exchange capacity and inner ion transfer efficiency of ionic groups within membranes. Generally, based on the electronic types of ionic groups attached on the reactive polymer, IEMs can be classified into cation exchange membranes (CEM) and anion exchange membranes (AEM). Cation exchange membranes contain negatively charged groups, such as $-\mathrm{SO}_{3},-\mathrm{COO}^{-},-\mathrm{PO}_{3} \mathrm{H}^{-},-\mathrm{C}_{6} \mathrm{H}_{4} \mathrm{O}^{-}$, etc., fixed to the reactive polymer chains and allow the passage of cations but reject anions. While anion exchange membranes contain positively charged groups, such as $\mathrm{NH}_{3}{ }^{+},-\mathrm{NRH}_{2}{ }^{+},-\mathrm{NR}_{2} \mathrm{H}^{+},-\mathrm{NR}_{3}{ }^{+},-\mathrm{PR}_{3}{ }^{+},-\mathrm{SR}_{2}{ }^{+}$, etc., run the opposite way [17]. 
Specifically, the cation exchange membranes are only permeable by cation. The anion exchange membranes perform the opposite way. Recently, the interests in applications of ion exchange membranes to solve two environmental issues have been aroused. One is enrichment and recovery of valuable ions, another is removal of undesirable ions from wastewater, such as plating wastewater treatment [10-12]. In this process, cation, such as nickel, copper, sodium etc., can exchanged with fixed ions attached on matrix.

Meanwhile, anions, such as sulfates, chromates and chlorides etc., are exchanged with hydroxyl ions [13-14].

The IEMs studied in this work are based on the electrodialysis' application for plating wastewater treatment. In this research, the ions conduction mechanism within polyolefinbased IEMs and the factors affecting membrane's physical properties and working performance were studied by quantitively analyzing the relationship of structure and properties

\subsection{Motivation}

For the generally application, a desirable membrane is required to possess of a stable inert polymer material and some continuous ionic channels across the film with a high ion exchange capacity (IEC) and good ion mobility, while maintaining its structural integrity and stability under some specific conditions $[5,6]$. The specific desirable properties of IEM are included:

i. High perm-selectivity - an ion-exchange membrane should be highly permeable to counter-ion, but impermeable to co-ions.

ii. High ion exchange capacity — an ion-exchange membrane should have high counter ions exchange capacity with the fixed ions on the polymer chains. That is the key factor of purifying solution.

iii. Low electrical resistance - ion-exchange membranes should have low electrical resistance and thus there will be less energy consume during electro-membrane processes.

iv. High chemical stability - the membrane should be stable over a $\mathrm{pH}$-range from 0 to 14 and under the presence of oxidizing agents.

v. Good mechanical stability - membrane should be mechanically strong and should have a low degree of swelling or shrinkage in transition from dilute to concentrated ionic solutions $[22,23]$.

During practical production, it is hard to accomplish all above membrane properties at the same time due to the antagonistic effect of above parameters on membrane properties. 
For instance, the more ionic charges attached on membranes matrix leads to a low electrical resistance, but causes the high-water uptake ability which would break the dimension stability; increasing the mechanical property will also increase the electro resistance of membranes which will lead the higher power consumption of electrochemical devices. Another conflict focused on homogeneous and heterogeneous ion exchange membranes. According to the connecting way of charge groups on the matrix or inert polymer, ion exchange membranes can be further classified into homogeneous and heterogeneous membranes, in which the charged groups are chemically bonded to or physically mixed with the membrane matrix, respectively $[5,19]$. Homogeneous ion exchange membranes have widely applications due to the excellent electrochemical performance. However, during the long service life, homogeneous ion exchange membranes cannot maintain the desirable mechanical properties and structure stability in a harsh environment, which requires a high structure and chemical stability for the membranes [20]. Whereas, the heterogeneous ion exchange membranes have good mechanical properties but weak electrochemical performance due to the hydrophilic polymer/ hydrophobic polymer's incompatibility and phase separation[21]. This phase separation also result the dimensional stability of homogeneous membrane is superior to heterogenous membranes. Thus, there is a compromise among these properties in the ionexchange membranes with high ion exchange capacity, good ionic conductivity, longterm chemical and structural stability.

The ion exchange membrane studied in this research aim at the electrodialysis application for plating wastewater treatment. This direction has not been extensively reported. The primary problem to impede ion exchange membrane extensively used in plating water treatment is that the plating wastewater contains plenty of heavy metals ions, oil, grease and suspended solids that might be hazardous to the ion exchange membrane. This harsh situation requires membrane possessing high structure and chemical stability during the long service life and over a wider $\mathrm{pH}$ range [14]. Attaching strongly acidic or basic ionexchange group, such as sulfone acid or trimethyl ammonium could be a promising way to make ion exchange membrane accommodated in this situation. However, the monomers containing a strongly acidic or basic ion-exchange group are difficult to graft directly onto hydrophobic polymers, because these highly ionizable groups with a large hydration sphere are incompatible with the hydrophobic inert polymers. This incompatibility will lead to the phase separation and further weaken electroconductivity, which is regards as one of the important properties of ion exchange membrane.

Another problem is related on the way of grafting monomers on backbone polymer. Backbone material is inert polymer and have no radical sites can be functionalized with ionic groups. It needs to be mortified to create radical sites to be grafted reactive polymers which can be functionalized by ionic groups. The most widely used polymerization method is radiation grafting method, followed by a functionalization reaction (sulfonation, alkaline hydrolysis of benzyl groups) [24]. The high penetration depth and strong energy are the main radiation characteristic, which can induce the 
radical reaction at the inner part of polymeric materials. However, the radiation induced copolymerization still exists some problems. Radiation-grafted membranes can be synthesized using two primary methods: (1) direct or simultaneous radiation grafting; and (2) pre-irradiation grafting. In the direct method, the backbone polymer is immersed in a pure monomer or monomer solution, followed by exposing to radiation [25]. During the radiation step, radical sites are generated to accept monomers by polymerization, followed attaching functional groups on the monomer chains. However, a potential limitation of direct radiation is side reactions, which leads to the formation of homopolymer and runs parallel reaction to grafting reaction [26]. In the pre-irradiation procedure, the backbone polymer is first irradiated under certain conditions to create radicals along the backbone, then the radiated backbone polymer is brought into contact with a monomer under controlled conditions. The main drawback of this method is that to achieve enough radicals, high irradiation doses are needed, which would lead to degradation of the polymer during the irradiation stage and consequent changes polymer's structure. Another issue is during long-term storage, the stability of the membrane radicals generated by irradiation is unwarrantable, as the radicals within the matrix can decay over time under increased temperatures [5]. Radical decay during storage will affect the subsequent grafting reaction and, consequently, affect the properties of the final membranes.

Compare to the radiation-induced graft polymerization, the chemical induced graft polymerization using chemical initiator to trigger the graft polymerization, is relatively safe and easy to control. Unfortunately, the reaction mechanism of initiator inducing graft polymerization and how the initiator induced membrane structure influence the final properties have not been intensively studied. Therefore, it's worthy to study the reaction mechanism of chemical induced graft polymerization for the polyolefin-based ion exchange membranes, and how it affects the final properties of membranes.

\subsection{Objectives}

Based on the above discussion, our research used the chemical initiator to create the radical sites of based polymer and induce polymerization with active monomers. In this research, we will investigate the effects of inert matrix structure, monomers addition, crosslinked degree and reaction conditions on ion exchange membranes properties. The properties to be studied include IEC, mechanical property, water uptake property, thermal expansion and electrochemical properties. The ion exchange membranes were expected to obtain high ion exchange capacity and selectivity, high conductivity and chemical resistance, and the desirable mechanical strength by means of quantitatively analyzing how the membrane structure, synthesizing conditions and multiple properties relate on each. The effects of fillers, such as GF and GO derivations, on properties of ion exchange membrane were investigated. The mechanical and electro conductivity were expected to be enhanced by adding modified GF and functionalized GO respectively. 


\section{Background}

\subsection{Literature Review}

Since 1970s, DuPont has developed a perfluoro sulfonic acid called "Nafion" that not only showed a two-fold increase in the specific conductivity of the membrane but also extended the lifetime by four orders of magnitude (104-105 h). This soon became a standard for proton exchange membrane fuel cell and remains so till today. Many commercial ion exchange membranes have been developed various functional based on the diversified applications. Table 1.1. and 1.2. provide a comparation of some commercial ion exchange membranes' properties [27]. As mentioned above, the typical properties of ion exchange membrane were determined by the backbone materials, monomers and synthetical conditions. We will review the recent progress in these three parts.

Table 1.1. Properties comparation of commercial cation exchange membranes

\begin{tabular}{llclll}
\hline $\begin{array}{l}\text { Membranes } \\
\text { models }\end{array}$ & $\begin{array}{l}\text { Membrane } \\
\text { types }\end{array}$ & $\begin{array}{c}\text { IEC emq } \\
\text { gr }^{-1}\end{array}$ & $\begin{array}{l}\text { Thickness } \\
\mathrm{mm}\end{array}$ & $\begin{array}{l}\text { Water } \\
\text { uptake } \%\end{array}$ & $\begin{array}{l}\text { Conductivity } \\
\mathrm{S} / \mathrm{cm} 30\end{array}{ }^{\circ} \mathrm{C}$
\end{tabular}

\begin{tabular}{|c|c|c|c|c|c|}
\hline \multicolumn{6}{|c|}{ Asahi chemical industry company Ltd, Japan } \\
\hline K101 & $\begin{array}{l}\text { Sulfonated } \\
\text { poly arylene }\end{array}$ & 1.4 & 0.24 & 24 & 0.0114 \\
\hline \multicolumn{6}{|c|}{ Asahi Glass company Ltd, Japan } \\
\hline CMV & $\begin{array}{l}\text { Sulfonated } \\
\text { poly arylene }\end{array}$ & 2.4 & 0.15 & 25 & 0.0051 \\
\hline DMV & $\begin{array}{l}\text { Sulfonated } \\
\text { poly arylene }\end{array}$ & & 0.15 & & 0.0071 \\
\hline
\end{tabular}

\section{Ionic chemical company, USA}

\begin{tabular}{lllll}
\hline MC 3470 & 1.5 & 0.6 & 35 & 0.0075 \\
\hline MC3142 & 1.1 & 0.8 & & 0.0114
\end{tabular}

Ionics Inc, USA

\begin{tabular}{lllll}
\hline 61AZL386 & 2.3 & 0.5 & 46 & 0.0081
\end{tabular}

\section{DuPont company, USA}




\begin{tabular}{llllll}
\hline N117 & $\begin{array}{l}\text { Per } \\
\text { fluorinated }\end{array}$ & 0.9 & 0.2 & 16 & 0.0133 \\
\hline N901 & $\begin{array}{l}\text { Per } \\
\text { fluorinated }\end{array}$ & 1.1 & 0.4 & 5 & 0.01053 \\
\hline \multicolumn{2}{l}{ Pall RAI Inc, USA } & & & & \\
\hline R-1010 & $\begin{array}{l}\text { Per } \\
\text { fluorinated }\end{array}$ & 1.2 & 0.1 & 20 & 0.0333 \\
\hline
\end{tabular}

Table 1.2. Properties comparation of commercial anion exchange membrane.

\begin{tabular}{|c|c|c|c|c|c|}
\hline $\begin{array}{l}\text { Monomer } \\
\text { types }\end{array}$ & $\begin{array}{l}\text { Polymer } \\
\text { backbones }\end{array}$ & $\begin{array}{l}\text { Ion } \\
\text { conductivity/m } \\
\text { S } \cdot \mathbf{c m}^{-1}\end{array}$ & $\begin{array}{l}\text { Water } \\
\text { uptake \% } \\
20{ }^{\circ} \mathrm{C}\end{array}$ & $\begin{array}{l}\text { IEC } \\
\text { Meq } \cdot g^{-1}\end{array}$ & $\begin{array}{l}\text { Mechanic } \\
\text { al } \\
\text { strength }\end{array}$ \\
\hline VBC/TMA & PVDP & & $70-98$ & 0.7 & $\begin{array}{l}\text { Very } \\
\text { brittle }\end{array}$ \\
\hline VBC/TMA & PEP & $10-20$ & $47-53$ & 1.0 & $\begin{array}{l}\text { TS 6-7 } \\
\mathrm{MPa}\end{array}$ \\
\hline VBC/TMA & PFA & 50 & $96-115$ & $1.38-1.61$ & \\
\hline VBC/TMA & PFA ETFE & $\begin{array}{l}\text { 52-87 PFA 48- } \\
\text { 69ETFE }\end{array}$ & $\begin{array}{l}38-78 \text { PFA } \\
42-81 \\
\text { ETFE }\end{array}$ & $\begin{array}{l}0.9-1.4 \\
\text { PFA } 1.1- \\
1.5 \text { ETFE }\end{array}$ & \\
\hline $\begin{array}{l}\text { Dimethyl } \\
\text { aminoethyl } \\
\text { methacrylat } \\
\text { e }\end{array}$ & ETFE & & & 1.62 & \\
\hline $\begin{array}{l}\text { Vinylbenzyl } \\
\text { trimethyl } \\
\text { Ammonium } \\
\text { chloride }\end{array}$ & ETFE & & 19.01 & 0.8 & \\
\hline VBC/TMA & ETFE & 27 & 40 & & $\begin{array}{l}\text { TS } \\
18.2 \mathrm{MPa}\end{array}$ \\
\hline $\begin{array}{l}\text { VBC/DAB } \\
\mathrm{CO}\end{array}$ & ETFE & 63 & 49 & 1.98 & TS51MPa \\
\hline $\begin{array}{l}\text { VBC Vinyl } \\
\text { pyridine }\end{array}$ & $\begin{array}{l}\text { LDPE } \\
\text { HDPE }\end{array}$ & & & $\begin{array}{l}0.763 \mathrm{LDP} \\
\mathrm{E}\end{array}$ & \\
\hline
\end{tabular}




\begin{tabular}{llll}
\hline & & & $1.317 \mathrm{HD}$ \\
& & & PE \\
\hline VBC/TMA & LDPE & $180-430$ LDPE & $1.35-2.13$ \\
& HDPE & $140-280 \mathrm{HDPE}$ & LDPE \\
& ETFE & & $1.88-1.97$ \\
& & & HDPE \\
& & & $0.82 \mathrm{ETFE}$ \\
\hline
\end{tabular}

\subsection{Inert polymer (backbone polymer) types of ion exchange membrane}

As the polymerization process involves the covalent attachment of a monomer to a backbone membrane, the nature of the backbone should play an important role. It is worth mentioning that various polymers have been explored to produce ion exchange membranes, including polystyrene [28,29], polyether sulfone, poly(phenylene oxide), poly(ether imide), poly(ether ketone), poly(benzimidazole), poly(vinyl alcohol) [30,31], chitosan [32], and fluorinated polymers [33-36]. In general, the materials used in synthesis of the ion exchange membranes can be classified into two vast groups: perfluorinated ionomers (or partially per-fluorinated), and nonfluorinated hydrocarbons (including aliphatic or aromatic structures).

\subsubsection{Fluorine-containing hydrocarbons-based membranes}

Fluorine-containing polymers are widely used as inert polymer due to their outstanding chemical stability (they are the basest resistant polymers) and thermal stability compared to hydrocarbon polymer-based matrices, their low surface energy, and adjustable properties using grafting method. The mainly fluoropolymer backbones can be divided into two categories: (1) per fluorinated polymers such as poly(tetrafluoroethylene-co hexafluoropropylene) (FEP), poly(tetra fluoroethylene-co- perfluoro propyl vinyl ether) (PFA), poly(tetrafluoroethylene) (PTFE) [37]; and (2) partially fluorinated polymers such as poly(vinylidene fluoride-co- hexafluoropropylene) (PVDF-co-HFP), poly(vinyl fluoride) (PVF), poly(vinylidene fluoride) (PVDF), poly(ethylene-alttetrafluoroethylene) (ETFE) [46], and poly(chlorotri-fluoroethylene) (PCTFE).

The fluorocarbon-based ion-exchange membranes (Nafion) with good chemical and thermal stability have been developed by DuPont [38]. The high equivalent weight (EW) of per-fluorinated membranes resulted limited using in fuel cells because they consume high power density. Similar polymers are Flemion produced by Asahi Glass and Aciplex$\mathrm{S}$ produced by Asahi Chemical. Among the two major types, the DuPont product is considered to be superior because of its high cation conductivity, good chemical stability and mechanical strength [39]. 
Varcoe et al. used the polymer ethyl tetra fluoro ethylene (ETFE) as their matrix, irradiating it in air with high-energy rays $\left(60^{\circ} \mathrm{C}\right)$ to produce active sites [40]. At least two types of radicals were generated in their irradiation process, $-\mathrm{CH}_{2} \mathrm{C} . \mathrm{HCF}_{2} \mathrm{CF}_{2}$ and ROO., depending on the atmosphere present during radiation (nitrogen and air, respectively). $\mathrm{A}$ highly stable functional ionomer, chloromethyl styrene (CMS), was then used to form quaternary ammonium groups. The resulting film exhibited an IEC of $0.92 \mathrm{meq} \cdot \mathrm{g}^{-1}$ (dry membrane) as well as high $\mathrm{OH}^{-}$conductivity, even at ambient temperature, making it a feasible membrane for alkaline $\mathrm{H}_{2} / \mathrm{O}_{2} \mathrm{PEM}$ fuel cells, which demonstrated a power density of $110 \mathrm{~mW} \cdot \mathrm{cm}^{-2}$ at a $60^{\circ} \mathrm{C}$.

Liu et al. synthesized a novel alkaline membrane by grafting copolymerized vinyl benzyl chloride (VBC) onto preirradiated poly (tetrafluoroethylene-co-perfluoro propyl vinyl ether) (PFA) film, followed by quaternization and alkalization [41]. The irradiated membrane exhibited a maximum ionic conductivity of $0.05 \mathrm{~S} \cdot \mathrm{cm}^{-1}$ and a maximum power density of $16 \mathrm{~mW} \cdot \mathrm{cm}^{-2}$ for a direct methanol fuel cell at $60^{\circ} \mathrm{C}$. Beom-Seok Ko et al. [42] used VBC to radiolytically graft onto both partially fluorinated ETFE and per fluorinated polymer PFA films. The grafted VBC films were treated with trimethylamine to prepare the alkaline membranes.

Many commercially available fluorinated polymers have been explored for use in radiation-induced grafting technology to create ion exchange membranes. However, several synthesis-related issues must be considered when using fluorinated polymers as backbones: (1) fluorinated polymers, especially partially fluorinated ones like polyvinylidene fluoride (PVDF), degrade easily under alkaline conditions [43], leading to the elimination of $\mathrm{HF}$ and the subsequent formation of $\mathrm{C}=\mathrm{C}$, and finally resulting in the breakdown of the main chains. This degradation was found to be instantaneous, leading to hydroxyl and carbonyl groups on the PVDF $[44,45]$ and inducing both a darkening of the membrane and a drop in its mechanical properties. (2) irradiation reduce the molecular weight of some matrices, such as PTFE. It also cause main-chain scission because the $\mathrm{C}-\mathrm{F}$ bond is stronger than the $\mathrm{C}-\mathrm{C}$ bond; and (3) the radiation grafting ratio cannot be controlled accurately.

\subsubsection{Nonfluorinated hydrocarbons based membranes}

Due to several disadvantages of fluorinated polymers discussed above, they may not be suitable polymer substrates for electrodialysis applications. Hence, many nonfluorinated hydrocarbon polymers have been explored instead. Although most of these are still far from meeting practical requirements, some typical polymers have been investigated. For example, Stoica et al. [48] used a poly (epichlorohydrin-allyl glycidyl ether) copolymer as their matrix to prepare polymer electrolyte membranes. The anion-conducting networks were obtained by incorporating two cyclic diamines, 1,4-diazabicyclo-[2.2.2]octane (DABCO) and 1-azabicyclo-[2.2.2]-octane (quinuclidine). Poly (phenylene oxide) 
(PPO) has emerged as one of the most promising polymers for the fabrication of anionexchange membranes due to its excellent physicochemical properties. In this regard, Nikoli et al. [52] fabricated a polyvinyl alcohol (PVA) matrix using a solution-cast method and a $\gamma$-irradiation crosslinking technique. In their work, PVA was first completely dissolved in boiling water bubbled with Ar, then this solution was exposed to g-rays $(60 \mathrm{Co})$ for irradiation at room temperature.

The non-fluorinated hydrocarbon polymers are also widely used in production of cation exchange membrane. Presently, one of the most promising routes to high-performance proton conducting polymer electrolyte membranes is the use of hydrocarbon polymers for polymer backbones [12]. The base polymers can be aliphatic or aromatic polymers having benzene ring structures in the polymeric backbone of membrane or in the bulky pendant groups. Hydrocarbon membranes provide some definite advantages over perfluorinated membranes. They are less expensive, commercially available and their structure permits the introduction of polar sites as pendant groups [10]. Hydrocarbon polymers containing polar groups have high water uptakes over a wide temperature range, but the absorbed water is restricted by the polar groups of polymer chains.

\subsubsection{Polyethylene-based ion exchange membrane}

Many literatures have mentioned that polyethylene as the most common polymeric material were used as the backbones material for preparing ion exchange membranes $[53,54,56]$. Compare with other polymer backbone material, polyethylene has the properties of high ductility, excellent chemical resistance and low cost. They are known to be very stable in severe environments [55].

Polyethylene based ion exchange membranes have been confirmed to exhibit a good combination of chemical stability, hydrophobicity, high crystallinity, negligible swelling, and good mechanical properties under harsh environments [57]. The introduction of some continuous ion conductive channels offered ion exchange membranes the well-defined hydrophilic ionic channels that ensure a high ion exchange capacity value, controllable water swelling [58]. Recently, low-density polyethylene (LDPE) and high-density polyethylene (HDPE) films have been used to prepare anion exchange membranes by radiation grafting [49.50]. However, the ionic conductivity of the resulting membranes was so far quite low compared with that of Nafion membranes, necessitating further improvement. Sunaga et al. [47] irradiated polyethylene with electric beams to generate free radicals, then used glycidyl methacrylate (GMA) and divinylbenzene (DVB) to graft the matrix. Min Zhang and Hyung Kyu Kim developed a high-performance polyethylenebased anion exchange membranes (AEMs) which induced from a nuclear hydrogen and obtained IEC $3.3 \mathrm{mmol} \cdot \mathrm{g}^{-1}$ [28]. Jan Schauer reported a preparation method of heterogeneous ion-exchange membranes based on sulfonated poly (1,4-phenylene sulfide) and linear polyethylene. Poly (1,4-phenylene sulfide) was sulfonated with chlorosulfonic acid in 1,2-dichloroethane, then mixed with linear polyethylene in various 
ratios and the resulting blends were press-molded at $150 \circ \mathrm{C}$ to obtain the membranes. The resulting ion exchange capacity is $2.38 \mathrm{mmol} \cdot \mathrm{g}^{-1}$ [59]. Sherazi et al. [51] prepared fuel cell membranes by radiation grafting of $\mathrm{VBC}$ onto polyethylene (PE) powder, followed by membrane fabrication, quaternization, and alkalization. They focused on grafting poly (-vinylbenzyl chloride) onto ultra-high molecular weight polyethylene (UHMWPE) powder by $60 \mathrm{Co}$ irradiation. The prepared membranes exhibited maximum $\mathrm{OH}-$ conductivities of $47.5 \mathrm{mS} \cdot \mathrm{cm}-1$ at $90{ }^{\circ} \mathrm{C}$ and $30 \mathrm{mS} \cdot \mathrm{cm}-1$ at $60{ }^{\circ} \mathrm{C}$. Tauqir A. Sherazi, Joon Yong Sohn and Young Moo Lee successfully use the radiation grafted method to produce the polyethylene-based anion exchange membranes for alkaline fuel cells [60]. They reported grafting the vinyl benzyl chloride on to the ultra-high molecular weight polyethylene powder by radiation grafting. The grafted powder was subsequently fabricated into membrane by melt pressing. The grafted polyethylene membranes were post functionalized with trimethylamine, followed by alkalization to obtain anionexchange membranes. The AEMs showed reasonably good chemical stability, as evidenced by the ion exchange capacity being maintained for a long duration, even in highly alkaline conditions. The membranes exhibited a maximum ionic conductivity of $47.5 \mathrm{mS} \cdot \mathrm{cm}^{-1}$ at $90 \circ \mathrm{C}\left(30 \mathrm{mS} \cdot \mathrm{cm}^{-1}\right.$ at $\left.60 \circ \mathrm{C}\right)$. Kevin J. T. Noonan, Kristina M. Hugar and Henry A. Kostalik reported a new class base-stable ion exchanged membranes-----phosphonium functionalized polyethylene based alkaline anion exchange membranes [61]. The alkaline stability of a tetrakis-(dialkylamino)phosphonium cation was evaluated and directly compared with that of benzyl trimethylammonium cation. They developed a new methodology for appending these delocalized phosphonium cations to polyethylene.

\subsubsection{Styrene-divinylbenzene-based ion exchange membranes}

Cation and anion-exchange membranes can also be synthesized by copolymerizing of styrene and divinylbenzene, followed by solfonaiton and amination respectively. Typically, the cation-exchange membrane was prepared by the sulfonation of polymer with chlorosulfonic acid or with concentrated sulfuric acid in dichloroethane using the silver sulfate as catalyst. The anion-exchange membrane was prepared by chloromethylation of the polymer followed by the amination. It was reported that the anion-exchange membrane prepared from copolymerization of the 4-vinylpyridine and divinylbenzene followed by quaternization with methyl iodide gives the membrane with good electrochemical properties but lacks in chemical stability. The novel anionexchange membranes were synthesized through copolymerization of 4-vinylpyridine, epichlorohydrin and aniline in the presence of benzoyl peroxide, followed by quaternization of methyl iodide using hexane as a solvent. These membranes exhibited good electrochemical properties and mechanical strength and can be used for the conversion of sea and brackish water into potable water, production of salt from seawater and used in chlor-alkali production. However, it was reported [60] that pure styrenedivinylbenzene based membranes have weak monovalent ion selectivity. 


\subsubsection{Polysulfone-based ion exchange membranes}

Engineering plastics such as polysulfone and polyether sulfone have been widely used as a base polymer for ultrafiltration and gas separation due to their excellent workability and mechanical strength [62]. A few studies have been reported to prepared ion exchange membrane using polysulfone as a base polymer due to its excellent chemical resistance. The polysulfone based ion exchange membrane has been applied in ultrafiltration and reverse osmosis to improve the permeability. The sulfonation procedure of polysulfonebased membrane were divided the solution procedure or the slurry procedure [63]. A new sulfonation process for polysulfone ionomers has been proposed $[65,66]$. The basic working hypothesis for the new type of sulfonation method was that poly(sulfone) is usually sulfonated at the ortho position to the ether bridge of the bisphenol-A-position by electrophilic substitution [60], because this part of the molecule has high electron density in contrast to the diary sulfone portion of the repeating monomer unit, which has a low electron-density due to the electron-withdrawing $\mathrm{SO}_{2}$ group. One disadvantage of sulfonated poly(sulfone) is that the $\mathrm{SO}_{3} \mathrm{H}$ group at this position can undergo substitution with $\mathrm{H}^{+}$, which lowers the hydrolytic stability of the sulfonated poly(sulfone) in strong acids. This limits the application of the commonly sulfonated poly(sulfone) as a cationexchange polymer in electro-membrane process. Thus, the new method was based on the sulfonated polysulfone in the diary sulfone part should show increased hydrolytic stability against ipso-substitution by $\mathrm{H}^{+}$compared to electrophilically sulfonated polysulfone, due to the electron-deficient of the diary sulfone position of the polymer. Based on the literature $[63,64]$, it was found that polysulfone can be substituted in the electron deficient portion of the monomer by first deprotonating the aromatic ring position ortho to the sulfone bridge with metalorganic reagents and subsequent reaction of the metalation site with the electrophiles. Deprotonation reactions by attack of metalorganics at the polymer backbones are also known from poly (2,6-dimethyl-paraphenylene ether) (PPO), poly(styrene), poly (vinyl thiophene) and poly (methylphenyl phosphorene). To convert the metalation site of the deprotonated polymer into sulfonic acid group, the metalation polymer is quenched with the electrophile $\mathrm{SO}_{2}$, and the metal sulfinate derivative of the respective polymer was formed. Sulfinates and particularly, the corresponding sulfonic acids were known to be chemically unstable and could easily be oxidized to sulfonic acids.

\subsection{Active monomers types of ion exchange membrane}

The functional ionic groups of ion exchange membrane mainly come from the monomers. In the literature, different monomers have been studied for the grafting process, including chloromethyl styrene, amino siloxane groups, hydrogenated olefins with an aliphatic ammonium, glycidyl methacrylate, trifluoro styrene, and vinyl imidazole. These grafting monomers can be classified into two categories: ionic monomers and neutral monomers. Normally, ionic monomers can be directly grafted onto the polymer backbone of the ion exchange membrane. For example, grafting an ionic 
vinyl monomer such as vinyl imidazole or vinyl benzyl trimethylammonium chloride [67] could directly grafted a cationic character upon the polymer substrate, which might be further tuned by substitution or complexation. Grafting of neutral monomers such as styrene (St) could result in copolymer precursors that might be chemically activated by a post-grafting activation reaction to introduce ionic characters [68]. Usually, a wide variety of functional monomers can be used to introduce ion-exchange groups onto the grafted polymeric chains via irradiation. VBC is widely applied as a grafting monomer in the radiation grafting process to fabricate anion exchange membranes. For example, the ionic conductivity of poly (FEP-g-VBC) was reported to be $0.023 \pm 0.001 \mathrm{~s} \cdot \mathrm{cm}^{-1}$ at 50 ${ }^{\circ} \mathrm{C}$, which was $20-50 \%$ of the values for Nafion- 115 when it was tested in a fuel cell [69]. Ko et al. [144] immersed an ETFE film in a bottle containing a mixture of vinyl benzyl chloride and chloroform, which was irradiated using $\delta$-rays, and the obtained ETFE-gPVBC film was subsequently immersed in a mixture of 1,4diazabicyclo[2,2,2]octane/dichloromethane to yield quaternization membranes [70].

Geng et al. [71] did similar work and successfully grafted VBC onto several fluoropolymer films, including FEP, PEA, and ETFE, using a simultaneous irradiation method. Elmidaoui et al. [72] used hydrogenated olefins with an aliphatic ammonium monomer and grafted this onto ETFE. Roualdes et al. [73] grafted amino siloxane groups onto a polyvinylidene fluoride (PVDF) backbone polymer using a low-frequency plasma deposition process in a tetramethylsilane/ammoniac mixture. Membranes were also prepared by grafting a,b,b-trifluorostyrene (TFS) or a,b,b-trifluorovinylnaphthylene (TFN) via the irradiation of PVDF, PE, ETFE, and PTFE films [74,75], which were then chloromethylated and quaternized via trimethylamine. Among these different membranes, the one grafted onto PE showed the lowest resistance, $4.4 \mathrm{U} \cdot \mathrm{cm}^{-2}$, with an IEC value of $0.86 \mathrm{meq} \cdot \mathrm{g}^{-1}$ although it was not specifically for fuel cell application, Acrylate monomers such as methyl acrylate (MAA) [75] and glycidyl methacrylate GMA $[76,77]$ were also adopted to form grafting copolymers that could be translated into cationic materials by means of post-grafting amination reactions. Vinyl imidazole was also chosen to be grafted onto poly (vinyl fluoride) (PVF), followed by quaternization. Nagarale et al. [78] grafted $\mathrm{SoCl}_{2}$ groups rather than monomers, using photochemical means in the presence of sulfur dioxide and chlorine. These groups enabled nucleophilic substitution of a diamine, followed by quaternization with ammonium to make a functionalized polyethylene. The monomer grafted to the backbone can also be composed of different components. Recently, Naguib et al. [79] calculated the reactivity ratio of vinyl imidazole and acrylic acid using different methods. They found that maximum grafting on polypropylene films using g-irradiation could be achieved with a monomer composition of $60 \%$ vinyl imidazole and $40 \%$ acrylic acid. 


\subsection{Fabrication methods of ion exchange membranes}

Generally, there are four methods for fabricating ion exchange membranes: (1) grafting polymerization method with using of the $\gamma$-ray irradiation, (2) grafting polymerization method with using of the plasma, (3) sole gel method and (4) direct polymerization of monomers. In follow, the above methods are explained in detail $[80,81]$.

\subsubsection{Radiation grafting polymerization method}

Graft polymerization, by means of electron-beam, $\gamma$-ray, and ultraviolet (UV) light irradiation or by plasma, is a convenient method for the preparation of ion exchange membranes because a rapid formation of active sites on an appropriate polymer matrix can be achieved $[82,83]$. The advantages of radiation-grafted membranes include the lack of need for chemical initiators or catalysts, the easy preparation from the already prefabricated base film. The grafting can be initiated by high-energy irradiation such as $\gamma$ ray, electron-beam and swift heavy ions. The base film in the polymer electrolyte membrane is a function as hydrophobic host that constrains the membrane swelling in water and provides the mechanical strength and dimensional stability. The process for the preparation of the new polymer electrolyte membrane by irradiation grafting as shown in Figure 2.1.in which three steps are as follows: In first step the polyethylene tetrafluoro ethylene (ETFE) films were pre-irradiated in argon gas at room temperature. In this step, the ETFE films were activated in a pre-irradiation step and then grafted with monomers in a subsequent step (second step) which is named substitution stage. Finally, the grafted ETFE films were sulfonated in a chlorosulfonic acid solution to introduce the sulfonic acid groups into the membranes which is followed by hydrolysis in distilled water.

Numerous studies on preparation of polyethylene ion-exchange membranes by radiationinduced graft polymerization have been reported. Satoshi Tsuneda and Kyoichi Saito reported a novel ion-exchange membrane containing sulfonic acid $\left(\mathrm{SO}_{3} \mathrm{H}\right)$ groups were prepared by a method of radiation-induced co-grafting of sodium styrene sulfonate (SSS) with acrylic acid (AAc) and vinyl benzyl trimethyl ammonium chloride (VBTAC) with 2-hydroxyethyl methacrylate (HEMA). The maximum cation exchange capacity of the resultant membranes was $2.5 \mathrm{mmol} \cdot \mathrm{g}^{-1}$ [69]. J.A. Horsfall, K.V. Lovell described the processes of simultaneous and pre-irradiation graft copolymerization of styrene to modify low density polyethylene and high-density polyethylene polymers and their sulfonation to produce hydrophilic membranes. The IEC are $1.7 \mathrm{mmol} \cdot \mathrm{g}^{-1}$ and $1.4 \mathrm{mmol} \cdot \mathrm{g}^{-1}$ respectively [84]. Richard Espiritu exploited using gamma radiation to fabricate polyethylene based alkaline anion exchange membranes. He studied the effects of film thickness, gamma radiation dose and monomer concentration to grafting degree and ion exchange capacity [85]. Mi-Lim Hwang's work showed the desired PVBC-grafted PEEK film can be prepared using a simultaneous irradiation grafting method, and that the degree of grafting of the film is largely influenced by the irradiation conditions [86]. 
However, the radiation induced process usually results in very complicated molecular structures, making it hard to obtain the desired properties which can be implemented in specific situation.

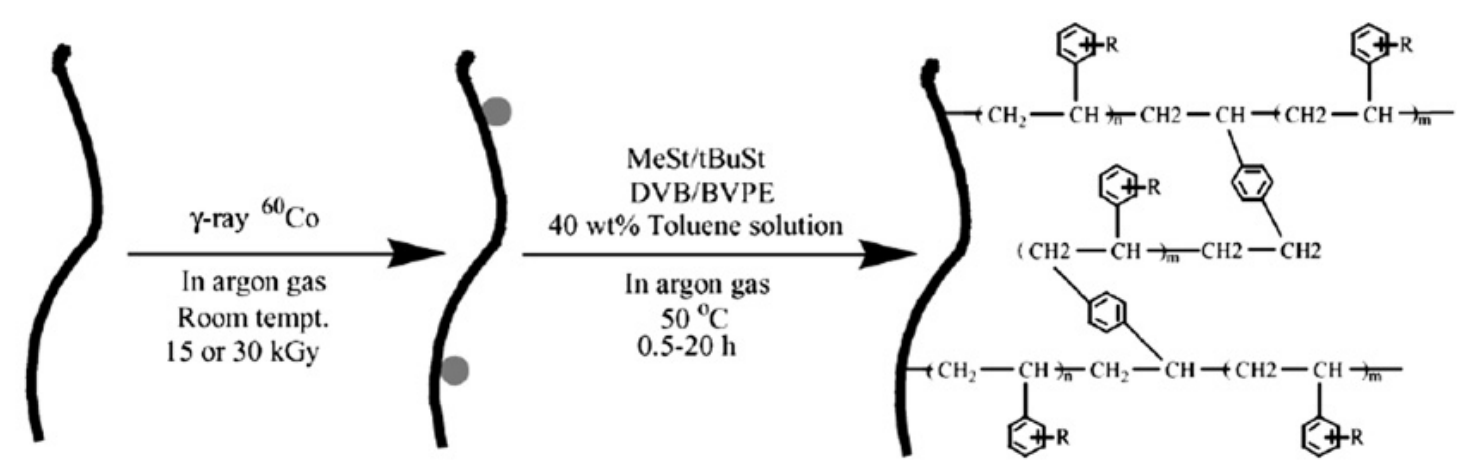

ETFE Film Pre-irradiated ETFE Film $\quad$ Grafted ETFE Film

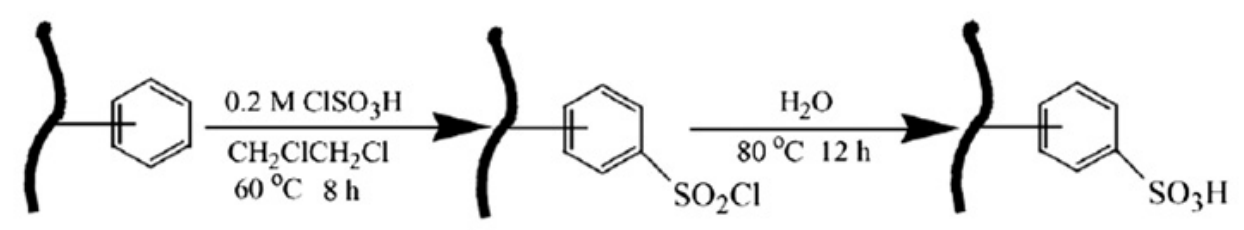

Grafted ETFE Film

Sulfonated ETFE Film Polymer electrolyte membrane

Figure 2.1. Preparation of the new polymer electrolyte membranes by irradiation grafting method. Image source: https://www.researchgate.net/figure/e-Preparation-of-the-newpolymer-electrolyte-membranes-by-irradiation-grafting-method_fig10_222897063

\subsubsection{Plasma grafting polymerization method}

The grafting polymerization by plasma is one of the methods for preparation of ion exchange membranes for applications in miniaturized fuel cells. Plasma polymerized films exhibit a high degree of cross linkage and are pinhole free even for films of only a few hundred nanometers in thickness, in contrast to conventionally polymerized films. Hence, a sharp reduction of the methanol permeability and a decrease in the resistance of a fuel cell electrolyte membrane is achieved by using plasma polymerized electrolytes. The overall membrane resistance are also reduced by plasma polymerization method due to the lower thickness of the ion exchange membrane (in about $1 \mathrm{~mm}$ ) [46]. By increasing the plasma energy in this polymerization, ionic conductivity of membrane will be reduced due to the higher degree of crosslinking, because the transferring of water molecules is so hard. The plasma polymerized electrolyte membranes have been developed by using tetrafluoro ethylene to generate the polymeric backbone and vinyl 
phosphonic acid to incorporate acid groups, which are responsible for the cation conductivity. Figure 2.2. has been shown the major differences between a monomer, a conventional polymer and the polymer prepared by plasma polymerization. The structure of the prepared polymer in plasma polymerization is a quietly dense which this matter caused to severely reduction of the methanol crossover in the usage of this polymer as a membrane in direct methanol fuel cells.

monomer

ccnventional polymer

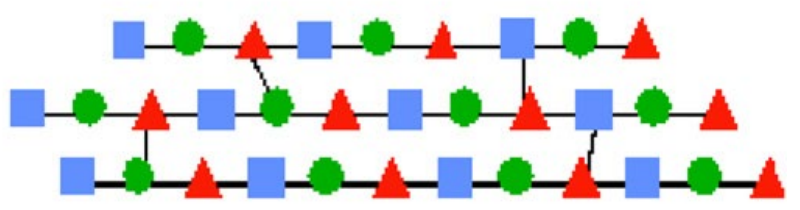

plasma polymer

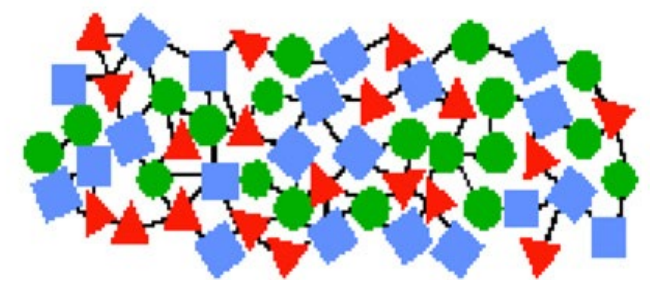

Figure 2.2. Differences between a monomer, a conventional polymer and the polymer prepared by plasma polymerization. Image resource:

https://www.researchgate.net/figure/e-Differences-between-a-monomer-a-conventionalpolymer-and-the-polymer-prepared-by_fig 12 _222897063

\subsubsection{Sol-gel method}

The sol-gel method provides the easier introduction of pure inorganic phase into polymeric matrix (mostly in composite membranes) [6]. In sol-gel chemistry, molecular pre-materials will change into particles with Nano sizes. This colloidal suspension form or sol leads to formation of the gel networks. Gel will change into different materials with the different properties by the various drying techniques. The sols have been formed from dispersing colloidal particles liquid and gel from rigid and continuous network with pores under micrometer size and polymer chains which have the average length greater than micron too. The sols are usually prepared by using of the metallic alkoxides. With regard to this, the metallic organics are insoluble in the water, but these alkoxides solved in the alcoholic solution. The polymerization reaction begins by adding of water in sol. This process will be done by two main reactions as hydrolysis and condensation. 


\subsubsection{Monomers direct polymerization method}

Direct polymerization of monomers is a new method of preparing ion exchange membranes. The membranes are prepared directly from the polymerization of possible monomers such as styrene and di-vinyl benzene, followed by sulfonation. The polymerization mostly takes place in an inert matrix through monomer soaking or pore can be filling. It has been noted that sometimes, the polymerization is directly conducted from sulfonated monomers without the post-sulfonation step [87]. Maria Gil has proved that the sulfonated aromatic poly (ether ether ketone) (S-PEEK) polymeric membranes could be directly synthesized from sulfonated monomer and evaluated for possible proton exchange membrane application. Rather than by radiation grafted polymerization, the chemical initiator was introduced to induce to radical polymerization by copolymerizing 4,4-difluorobenzophenone and fuming sulfuric acid to form 5,5-carbonylbis monomers and then form S-PEEKs through the co-condensation reaction. The synthesized S-PEEK membranes exhibit conductivities from 0.02 to $0.07 \mathrm{~S} / \mathrm{cm}$, water swelling from $13 \%$ to $54 \%$, ion-exchange capacities (IEC) from 0.7 to $1.5 \mathrm{meq} \cdot \mathrm{g}^{-1}$ and methanol diffusion coefficients from $3 \times 10^{-7}$ to $5 \times 10-8 \mathrm{~cm}^{2} \cdot \mathrm{s}^{-1}$ at $25^{\circ} \mathrm{C}$. These diffusion coefficients are much lower than that of Nafion $\left(2 \times 10^{-6} \mathrm{~cm}^{2} \cdot \mathrm{s}^{-1}\right)$, making S-PEEK membranes a good alternative to reduce problems associated with high methanol crossover in direct methanol fuel cells.

\section{5 lons conducting mechanisms in ion exchange membrane}

\subsubsection{Cation conduction mechanisms}

Cation conduction is fundamental for cation exchange membrane and is usually the first characteristic considered when evaluating membranes for electrodialysis using. Resistive loss is proportional to the ionic resistance of the membrane and high conductivity is essential for the required performance especially at high current density. At a molecular level, the cation transport in hydrated polymeric matrices is in general described based on either of the two principal mechanisms: (1) "ion hopping" or "Grotthus mechanism" and "diffusion mechanism" which water is as vehicle or "vehicular mechanism" [88].

In ion hopping mechanism cation hop from one hydrolyzed ionic site $\left(\mathrm{SO}_{3} \cdot \mathrm{H}_{3} \mathrm{O}^{+}\right)$to another across the membrane. In this mechanism, ionic clusters were swelled in presence of water and formed the percolation mechanism for cation transferring [89]. The simple scheme of the hopping mechanism has been shown in Figure 2.3. The hopping mechanism has little contribution to conductivity of per fluorinated sulfonic acid membranes such as Nafion. 


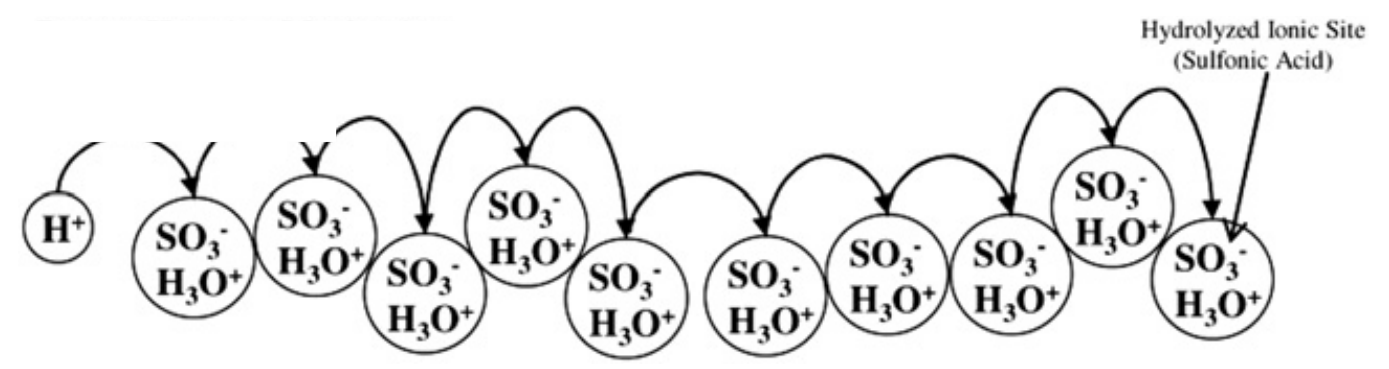

Figure 2.3. The simple scheme of the hopping mechanism. Image resource:

https://www.researchgate.net/figure/e-The-simple-scheme-of-the-hopping-mechanismreprinted-with-permission-from-16_fig4_222897063

The second mechanism is a vehicular mechanism. In this mechanism hydrated cation $\left(\mathrm{H}_{3} \mathrm{O}^{+}\right)$diffuses through the aqueous medium in response to the electrochemical difference. In vehicular mechanism, the water connected cation $\left(\mathrm{H}^{+}\left(\mathrm{H}_{2} \mathrm{O}\right) \mathrm{x}\right)$ in the result of the electroosmotic drag carry the one or more molecules of water through the membrane and itself are transferred with them. The major function of the formation of the vehicular mechanism is the existence of the free volumes within polymeric chains in cation exchange membrane which allow the transferring of the hydrated ions through the membrane. The schematic design of the vehicular mechanism in cation conduction in pristine membranes has been shown in the Figure 2.4.

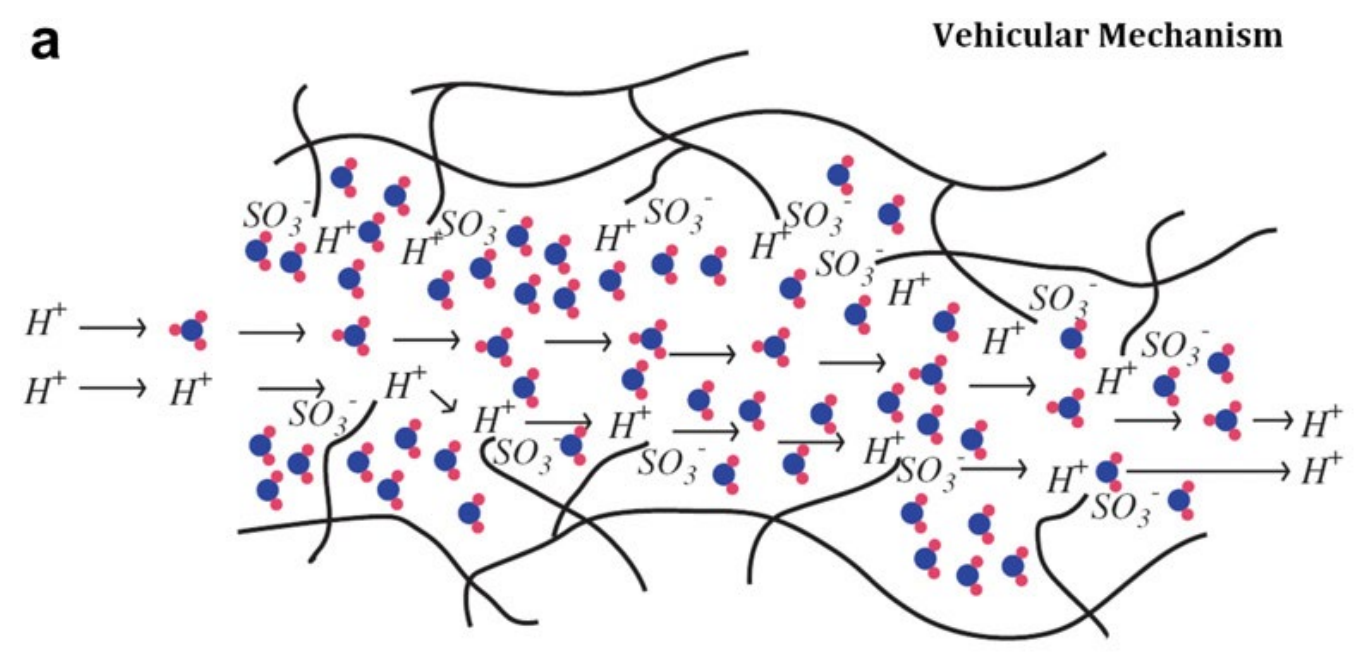

Figure 2.4. The Schematic design of the Vehicular Mechanism as cation conduction in pristine membranes

\subsubsection{Anion conduction mechanism}

$\mathrm{OH}^{-}$conduction is usually the major characteristic considered when evaluating anion exchange membranes for potential fuel cell use. Therefore, it is necessary to understand 
and be able to predict ionic transport and conduction in an anion exchange membrane as a function of relative humidity and other membrane properties. With respect to this, an effective approach is to use modeling and simulations to understand $\mathrm{OH}^{-}$transport. For example, Grew [90] described a dusty fluid model and used it to predict anion exchange membrane conductivities as a function of relative humidity and other membrane properties. A percolation model was constructed and used to account for the effect of membrane structure on ionic conduction. Kiss et al. [256] carried out a permeation experiment to measure water flux through a membrane. In each case, ionic conductivity could be predicted using either the measured water flux data or the dusty fluid model.

In general, in constructing models, the cation-conducting mechanism in Nafion membrane has been used as the reference point. It was assumed that the hydroxide transport mechanisms in anion exchange membranes were analogous to the cation transport mechanisms in cation-exchange membranes. $\mathrm{OH}^{-}$transport in an anion membrane can be divided into three categories (Figure 2.5): (1) the Grotthuss, or $\mathrm{OH}^{-}$ hopping, mechanism; (2) the diffusion and migration, or vehicular, mechanism; and (3) convection within the anion membrane [91]. It is generally recognized that the majority of $\mathrm{OH}^{-}$is transported within the anion membrane through the Grotthuss mechanism. The hydroxide ions move along a chain of water molecules by means of hydrogen bond formation and deformation. Diffusion and convection also play important roles in $\mathrm{OH}^{-}$ transport. Diffusive transport occurs at the concentration or electrical potential gradient. Convective transport of $\mathrm{OH}^{-}$across a film can drag water molecules with the ions, generating a convective flow of water molecules within the membrane. However, since the materials and structures of anion and cation membranes are different, the mechanisms of $\mathrm{OH}^{-}$and $\mathrm{H}^{+}$transport should different, as demonstrated by their different transport coefficients (at $25^{\circ} \mathrm{C}$ in water, the transport coefficient of $\mathrm{OH}^{-}$is about $5.3 \times 10^{-9} \mathrm{~m}^{2} \cdot \mathrm{s}^{-1}$, while that of $\mathrm{H}^{+}$is $9.3 \times 10^{-9} \mathrm{~m}^{2} \cdot \mathrm{s}^{-1}$ ). The dusty fluid model can provide valuable information about $\mathrm{OH}^{-}$transport mechanisms and strategies to improve membrane performance. 


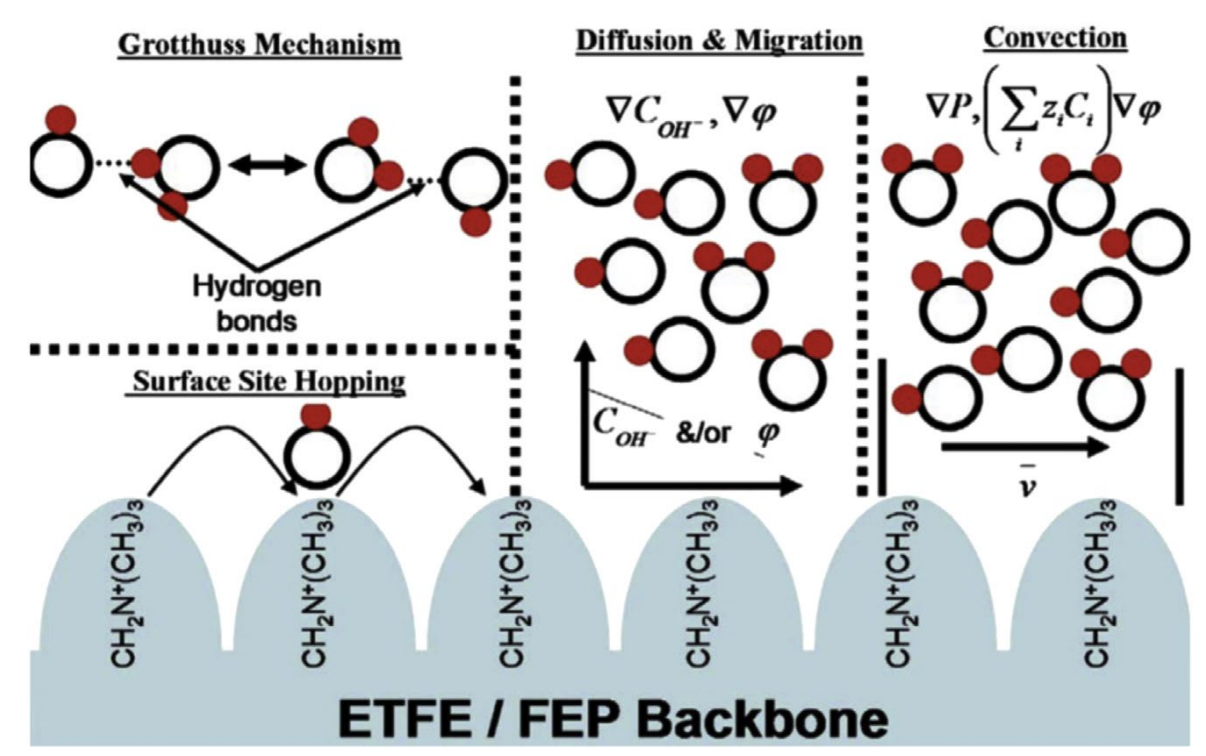

Figure 2.5 The three dominant transport mechanisms for hydroxide in alkaline membranes [91]. 


\section{Materials and experimental techniques}

\subsection{Materials}

\subsubsection{Materials for cation exchange membrane and anion exchange membrane}

Low-density polyethylene (LDPE), high high-density polyethylene (HDPE) and linear low-density polyethylene (LLDPE) films were supplied by Uline Co. Ltd. Styrene (99.9\% purity), divinylbenzene (DVB, 80\% purity), Luperox A98 (benzoyl peroxide, 98\%), 1,2-dichloroethane, chlorosulfonic acid, polystyrene-block-poly-(ethylene-ranbutylene)-block-polystyrene were purchased from Sigma Aldrich Co, Ltd. Toluene and xylene were supplied by Carolina Co, Ltd. Vinyl benzyl chloride (VBC 90\%), trimethylamine solution $(45 \mathrm{wt} \%$ in water) were purchased from Sigma-Aldrich. Sodium nitrate $\left(\mathrm{NaNO}_{3}\right)$, hydrochloric acid $(\mathrm{HCl}, 37 \%)$ and silver nitrate $\left(\mathrm{AgNO}_{3}\right)$ were supplied by Carolina Co, Ltd. Tetrahydrofuran (THF) was supplied by Fisher Chemical. All solvents were used as received.

\subsubsection{Fillers/ion exchange membrane composite}

\subsubsection{Glass fiber/ion exchange membrane composite}

1,6-bis (trimethoxysilyl) hexane (1,6 bis) was supplied by Gelest Inc; [3(Methacryloxy)propyl] trimethoxy silane (3-MPS) and Poly(propylene-graft-maleic anhydride) (PP-g-MA, maleic anhydride 8-10\%) were supplied by Sigma Aldrich Co, Ltd.; Triethoxyvinylsilane (TES) was purchased from TCI Co, Ltd.; glass fiber (GF) was supplied by Fibre Glast Developments Corporation(U.S.A), with diameter of 16 micron and average length of 230 micron. Hydrochloric acid ( $\mathrm{HCl}, 37 \%$ purity) was supplied by Carolina Co, Ltd. All solvents were used as received.

\subsubsection{Graphene oxide/ cation exchange membrane composite}

Synthetic graphite powder(particle size $<20 \mu \mathrm{m}$ ), concentrated sulfuric acid $(95 \%-98 \%)$, (3-Aminopropyl) trimethoxy silane (97\%), sulfanilic acid (99\%) and phosphoric acid $\left(\mathrm{H}_{3} \mathrm{PO}_{4}, 99 \%\right)$ were supplied by Sigma Aldrich; Sodium nitrate $\left(\mathrm{NaNO}_{3}\right)$, potassium permanganate $\left(\mathrm{KMnO}_{4}, 2.7 \mathrm{~g} / \mathrm{cm}\right)$ were purchased from PubChem; 30\% hydrogen peroxide and formaldehyde solution (37\%) were purchased from LabChem. Tetrahydrofuran (THF) was supplied by Fisher Chemical. 


\subsection{Experimental techniques}

\subsubsection{Cation exchange membrane preparation}

\subsubsection{Synthesis of PE-graft-PS copolymer}

PE films were rinsed with acetone and dried at $50{ }^{\circ} \mathrm{C}$ for $6 \mathrm{~h}$ to remove moisture. Then, 2 $\mathrm{g}$ PE was dissolved in mixed a solvent composed of $20 \mathrm{~mL}$ toluene and $2 \mathrm{~mL}$ xylene at $90{ }^{\circ} \mathrm{C}$. After completely dissolving, the styrene, DVB and BPO were added into the above mixture in nitrogen atmosphere. The reaction was kept at $90{ }^{\circ} \mathrm{C}$ for $6 \mathrm{~h}$. The mass ratio between PE and styrene was 1:1.5, the DVB and benzoyl peroxide were added in at a weight ratio of $3 \%$ and $0.5 \%$ to styrene respectively. After the reaction completing, the thermal plastic elastomer, polystyrene-block-poly-(ethylene-ran-butylene)-blockpolystyrene was blended with the PE-g-PS copolymer to increase the ductility. The mass ratio of PE-g-PS copolymer and elastomer were 5:1. Then, the viscous copolymer liquid was cast onto a piece of glass and slicked by a hot press machine to prepare the membrane.

\subsubsection{Sulfonation of PE-g-PS copolymer for cation exchange membrane preparation}

Sulfonation of PE-g-PS copolymer was carried out by immersing it in the chlorosulfonic acid solution (5\% chlorosulfonic acid mixed with $95 \%$ 1,2-dichloroethane) at $0{ }^{\circ} \mathrm{C}$ for 2 h. After sulfonation, the resulting membrane was repeatedly rinsed with distilled water to remove residual chlorosulfonic acid, and the cation exchange membrane composite was obtained after drying in an oven at $50{ }^{\circ} \mathrm{C}$ overnight.

\subsubsection{Anion exchange membrane}

\subsubsection{Synthesis of LDPE-graft-Poly (St-co-VBC) copolymer}

The LDPE-graft-Poly (St-co-VBC) copolymer was synthesized by a modified method described 3.2.1.1 for PE-graft-PS synthesis. In a typical synthesis, $5 \mathrm{~g}$ of LDPE films was dissolved in a mixture of $20 \mathrm{~mL}$ toluene and $2 \mathrm{~mL}$ xylene to stir. The reactor was flushed with nitrogen and heated to $90{ }^{\circ} \mathrm{C}$. After the LDPE films completely dissolved, $5.13 \mathrm{~mL}$ (32.8 mmol) VBC, styrene and $0.18 \mathrm{~mL}(0.98 \mathrm{mmol})$ divinylbenzene were added to the mixture followed by addition of $0.03 \mathrm{~g}(0.12 \mathrm{mmol})$ radical initiator BPO. The crosslinker (divinylbenzene) to monomer $(\mathrm{VBC}+\mathrm{St}$ ) ratio was kept at $3 \%$ for all graft copolymerization reactions. The reaction was kept at $90 \circ \mathrm{C}$ for $6 \mathrm{~h}$. The viscous composite liquid was cast onto a piece of glass and slicked by a hot press machine to 
prepare the membrane. To remove self-polymerized products, the solid film was cut into pieces and soaked with THF at $40{ }^{\circ} \mathrm{C}$ for $6 \mathrm{~h}$.

\subsubsection{Amination of LDPE-graft-Poly (St-co-VBC) for anion exchange membrane preparation}

Amination of LDPE-graft-Poly (St-co-VBC) was carried out by soaking LDPE-graftPoly (St-co-VBC) copolymer in trimethylamine solution ( $45 \mathrm{wt} \%$ in water) at $40 \circ \mathrm{C}$ for $24 \mathrm{~h}$ followed the well-known procedure. After reaction, the membrane was repeatedly washed with distilled water to remove trimethylamine residue. The resulting membrane was soaked in $1 \mathrm{~mol} \cdot \mathrm{L}^{-1} \mathrm{KOH}$ at room temperature for $24 \mathrm{~h}$ to give the $\mathrm{OH}^{-}$form of anion exchange membrane. The dried membrane product was obtained after drying in an oven at $50 \circ \mathrm{C}$ overnight.

\subsubsection{Glass fiber/ion exchange membrane composite preparation}

\subsubsection{Surface treatment of glass fiber}

The coupling agents were hydrolyzed in ethanol alcohol solution, adjusting $\mathrm{pH}$ to 3.5 using diluted hydrochloric acid. The concentration of coupling agents in ethanol solution is $2 \%$. After $1 \mathrm{~h}$ hydrolyzation, GF was immersed in the different coupling agent solutions for $1 \mathrm{~h}$ at $100{ }^{\circ} \mathrm{C}$. Then the glass fibers were dried at room temperature for $24 \mathrm{~h}$. The chemical structure and hydrolyzation mechanism of these four coupling agents are illustrated in Scheme 3.2.

When the effect of the coupling agent's concentration on the mechanical properties of composite was investigated, the concentration in ethanol solution varied to $1 \%, 3 \%, 4 \%$, $5 \%$ respectively.

1. 1. 1,6-bis (trimethoxysilyl) hexane (1,6 bis)<smiles>CO[Si](CCCCCC[Si](OC)(OC)OC)(OC)OC</smiles><smiles>O[Si](O)(O)CCCCCC[Si](O)(O)O</smiles>

2. [3-(Methacryloxy) propyl] trimethoxy silane (3-MPS)<smiles>C=C(C)C(=O)OCC[Si](O)(O)O</smiles>

3. Triethoxyvinylsilane (TES) 
<smiles>C=C[Si](O)(O)CCCCCCCC</smiles>

4. Poly (propylene-graft-maleic anhydride) (PP-g-MA)

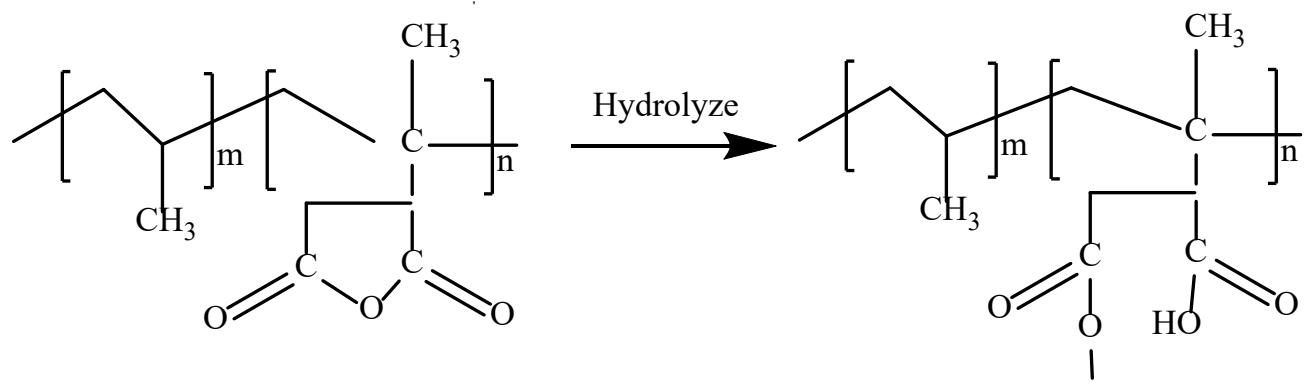

Scheme 3.2. Chemical structure of coupling agents and their hydrolyzation mechanism

\subsubsection{Synthesis and sulfonation of PE-graft-PS/GF membrane composite}

The synthesis of PE-graft-PS copolymer was described as 3.2.1.1. When the reaction was completed, the copolymer was stirred with the GF at $200{ }^{\circ} \mathrm{C}$ for $20 \mathrm{~min}$. The formulation of composite with untreated GF, treated GF and GF treated by different coupling agents' concentration were summarized in Table 1. The thermal plastic elastomer, polystyreneblock-poly-(ethylene-ran-butylene)-block-polystyrene was blended with the GF/PE-g-PS composite to increase the ductility. The viscous composite was then cast onto a piece of glass and slicked by a hot press machine to make the GF evenly distributed on the membrane. All the composites were carefully prepared under the same processing condition as above. The whole procedure is shown as Schematic 3.3.

Table 1. Formulation of composite

\begin{tabular}{|c|c|c|c|c|c|c|c|}
\hline \multirow[t]{2}{*}{ Sample } & \multirow{2}{*}{$\begin{array}{l}\text { PE- } \\
\text { g-PS } \\
(\%)\end{array}$} & \multirow[t]{2}{*}{$\begin{array}{l}\text { GF } \\
(\%)\end{array}$} & \multirow[t]{2}{*}{$\begin{array}{l}\text { Elastomer } \\
(\%)\end{array}$} & \multicolumn{4}{|c|}{$\begin{array}{l}\text { Concentration of coupling agent } \\
\text { treated on GF }\end{array}$} \\
\hline & & & & $\begin{array}{l}1,6 \\
\text { bis } \\
(\%) \\
\end{array}$ & $\begin{array}{l}\text { 3-MPS } \\
(\%)\end{array}$ & $\begin{array}{l}\text { TES } \\
(\%)\end{array}$ & $\begin{array}{l}\text { PP-g- } \\
\text { MA } \\
(\%) \\
\end{array}$ \\
\hline pure membrane & 83 & 0 & 17 & & & & \\
\hline $\begin{array}{l}\text { Composite with } 1,6 \text { bis bis } \\
\text { treated GF }\end{array}$ & 71 & 14 & 14 & 2 & & & \\
\hline \multirow{3}{*}{$\begin{array}{l}\text { Composite with 3-MPS treated } \\
\text { GF }\end{array}$} & 71 & 14 & 14 & & 1 & & \\
\hline & 71 & 14 & 14 & & 2 & & \\
\hline & 71 & 14 & 14 & & 3 & & \\
\hline
\end{tabular}




\begin{tabular}{lllllll}
\hline & 71 & 14 & 14 & 4 & & \\
\cline { 2 - 7 } & 71 & 14 & 14 & 5 & \\
\hline Composite with TES treated GF & 71 & 14 & 14 & & 2 \\
\hline $\begin{array}{l}\text { Composite with PP-g-MA } \\
\text { treated GF }\end{array}$ & 71 & 14 & 14 & & 2 \\
\hline
\end{tabular}

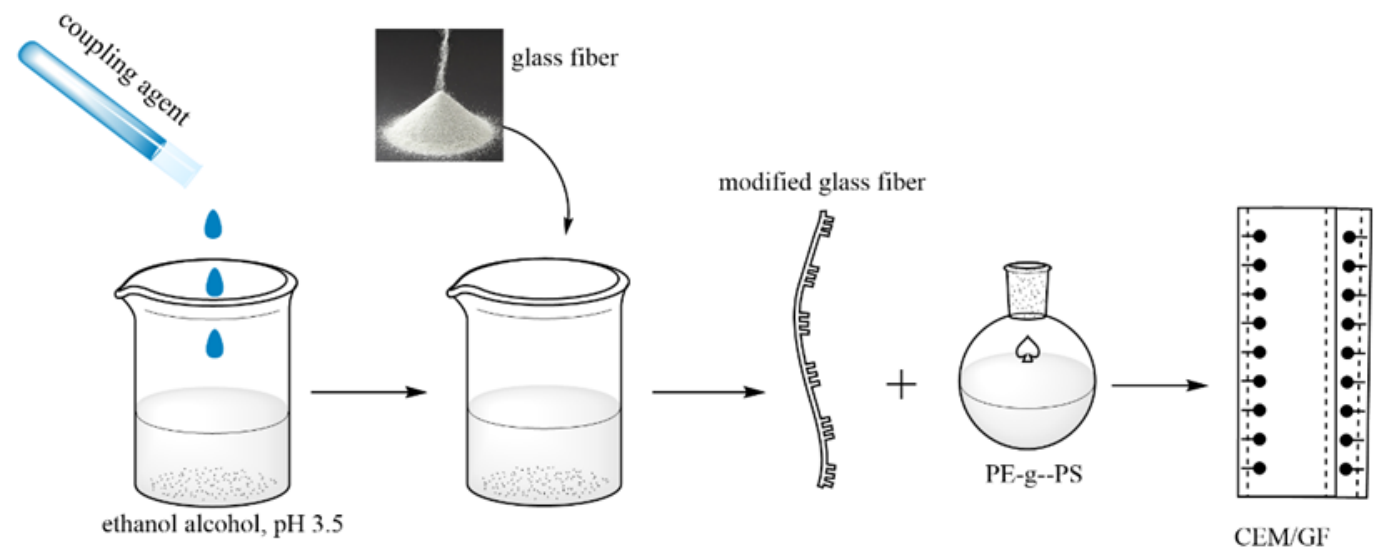

Schematic 3.2. Procedure of preparing glass fiber/cation exchange membrane (GF/CEM) composite

\subsubsection{Graphene oxide derivate/cation exchange membrane composite preparation}

\subsubsection{Preparation of graphene oxide (GO)}

GO is prepared from powder graphite following the Hummers procedure with modification. Typically, $2 \mathrm{~g}$ graphite powder and $2.5 \mathrm{~g} \mathrm{NaNO}_{3}$ were stirred with the 150 $\mathrm{mL}$ concentrated $\mathrm{H}_{2} \mathrm{SO}_{4}$ in ice bath for $30 \mathrm{~min}$. The obtained mixture was mixed with 15 $\mathrm{g} \mathrm{KMnO}_{4}$, controlling the temperature below $20{ }^{\circ} \mathrm{C}$. The reaction mixture was then stirred overnight at room temperature. Under vigorous stirring, the $180 \mathrm{~mL}$ deionized water was poured into mixture, followed by reflux at $98{ }^{\circ} \mathrm{C}$ for 24 hours. The reaction was stopped by adding $80 \mathrm{~mL} \mathrm{H}_{2} \mathrm{O}_{2}(30 \%$ concentration) to cool them down to room temperature. The obtained products are $\mathrm{GO}$, followed by rinsing and centrifugation with $5 \% \mathrm{HCl}$ and deionized water several times.

\subsubsection{Preparation of aminopropyl silane graphene oxide (MGO)}

GO can be modified by condensation reacting with (3-Aminopropyl) trimethoxy silane. In a typical synthetic procedure, $0.5 \mathrm{~g} \mathrm{GO}$ and $0.5 \mathrm{~mL}$ (3-Aminopropyl) trimethoxy silane were dissolved in $500 \mathrm{~mL}$ anhydrous THF and sonicated for $30 \mathrm{~min}$. The obtained 
homogeneous dispersion (MGO) was refluxed at $70{ }^{\circ} \mathrm{C}$ for $15 \mathrm{~h}$, followed by filtering and rinsing several times with THF, and dried to room temperature.

\subsubsection{Preparation of sulfanilic acid modified MGO (S-MGO)}

The MGO can be further modified by acid to add anionic group on it. Specifically, the $0.2 \mathrm{~g} \mathrm{MGO}$ was dispersed in $100 \mathrm{~mL}$ pure water and sonicated under $40 \mathrm{kHz}$. Then, the obtained mixture was stirred with $48 \mathrm{mg}$ sulfanilic acid in oil bath at $60{ }^{\circ} \mathrm{C}$ for $12 \mathrm{~h}$. After reaction, the mixture was filtered and washed several times with pure water to obtain $\mathrm{S}$ MGO.

\subsubsection{Preparation of phosphorous acid and sulfanilic acid modified MGO (PS- MGO)}

The MGO was dispersed in 1:1(w/w) formaldehyde and phosphorous acid solution and stirred for $3 \mathrm{~h}$ at $70{ }^{\circ} \mathrm{C}$, followed by washing with water and dried to room temperature to obtain phosphonic acid propylsilane graphene oxide (P-MGO). The P-MGO was then modified by sulfanilic acid to obtain PS-MGO under the same procedural as describing in 3.2.4.3.

\subsection{Sample characterizations}

\subsubsection{Fourier-transform infrared spectroscopy (FT-IR) analysis}

The grafted monomers, functional groups of the matrix membranes and final composite were investigated by FT-IR. A PerkinElmer Spectrum One FTIR spectrometer with universal attenuated total reflection accessory was used to record the infrared spectrum.

\subsubsection{X-ray diffraction (XRD) analysis}

XRD was used to characterize the graphene oxides and its derivate, such as GO, M-GO, PS-MGO, S-MGO. The interlayer spacing expansion of them can be investigated through calculating the shift of $2 \theta$ in XRD pattern. XRD patterns of samples performed using a Phillips X-ray diffractometer with $\mathrm{CuK} a\left(\lambda_{\mathrm{K} a}=1.5418 \AA\right)$ as the radiation source.

\subsubsection{Morphology characterization}

The microstructure, compatibility of filter and matrix membrane and phase separation of membrane composites were characterized by Field Emission Scanning Electron Microscope (FE-SEM) and transmission electron microscope (TEM) analysis. The Hitachi S-4700 FE-SEM was equipped with Oxford energy dispersive X-ray 
spectroscopy (EDS) microprobe. The TEM is a FEI 200kV Titan Themis Scanning Transmission Electron Microscope (S-TEM).

\subsubsection{X-ray photoelectron spectrometer characterization}

The chemical composition and chemical bonding formed by grafted polymerization and condensation reaction of samples were determined by X-ray photoelectron spectrometer (XPS) using a PHI 5800 X-ray spectrometer, using an Al K a X-ray source.

\subsection{Experimental test method}

\subsubsection{Mechanical properties}

The evaluation of mechanical properties to cation/anion exchange membrane and fillers/ ion exchange membrane composite include dynamic mechanical analysis (DMA), tensile strength test and burst strength. These tests can be obtained from DMA Q800 machine (Figure 3.1) and Zhibang Mullen burst test machine. The DMA includes storage modulus and loss factor test, being monitored from $25^{\circ} \mathrm{C}$ to $150{ }^{\circ} \mathrm{C}$, at a heating rate of $3.00{ }^{\circ} \mathrm{C}$. $\min ^{-1}$. Tensile strength was tested at the loading rate $0.5000 \mathrm{~N} \cdot \min ^{-1}$ until $5.0000 \mathrm{~N}$. The burst strength was conducted as following procedure: A sample membrane sheet (about $6 * 6 \mathrm{~cm}$ width) is fixed into a Mullen tester. The maximum pressure is the bursting strength when the membrane busts. The sample should keep moist condition before testing due to its shrink in dry condition.

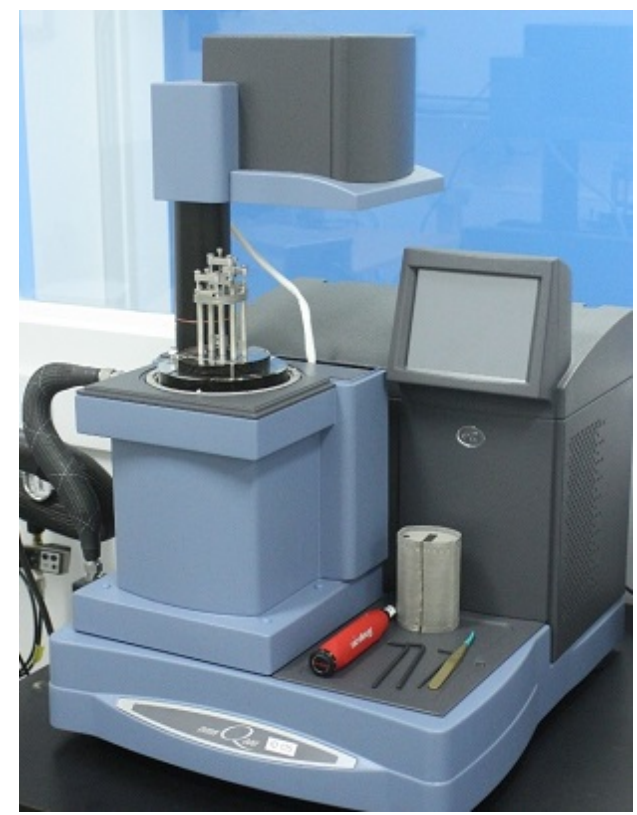

Figure 3.1. Dynamic mechanical analysis (DMA) Q800 machine 


\subsubsection{Ion exchange capacity (IEC)}

The IEC of the membrane is a key factor that determines the performance of the membrane in wastewater. IEC can calculate the moles amounts of the exchanged ions directly. Using this method can quantitatively analyze how component and reaction conditions influence IEC values. The IEC of ion exchange membrane was determined by titration method. The IEC of cation exchange membrane was determined by cutting membrane into small pieces and stirring with $1.0 \mathrm{M}$ sulfuric acid solution overnight to make sure sulfonate groups are in $\mathrm{H}^{+}$form. Then, the membranes were washed with distilled water to remove the excess sulfuric acid. The resulting membranes were dried in the oven at $60{ }^{\circ} \mathrm{C}$ and then stirred with $50 \mathrm{~mL} 0.5 \mathrm{M} \mathrm{NaCl}$ solution overnight to convert to sodium form (Scheme 3.3.). Due to the replacement of $\mathrm{H}^{+}$on the membrane by sodium cation from the solution, the solution becomes acidic. The concentration of $\mathrm{H}^{+}$was determined by $\mathrm{pH}$ measurement and titration using diluted solution (e. g. $0.01 \mathrm{M} \mathrm{KOH}$ ). The titration instrument is shown at Figure 3.2. The IEC values were calculated using the formula (1):

IEC $\left(\mathrm{mmol} \cdot \mathrm{g}^{-1}\right)=\left(\mathrm{V}_{\mathrm{KOH}} \times \mathrm{C}_{\mathrm{KOH}}\right) / \mathrm{W}_{\mathrm{dry}}$

where, $\mathrm{V}_{\mathrm{KOH}}$ is the volume of $\mathrm{KOH}$ used in the titration, and $\mathrm{W}_{\mathrm{dry}}$ is the dry weight of the membrane in $\mathrm{g}$. $\mathrm{C}_{\mathrm{KOH}}$ is the molarity $\left(\mathrm{mol} \cdot \mathrm{L}^{-1}\right)$ of $\mathrm{KOH}$ used in the experiment for the titration.

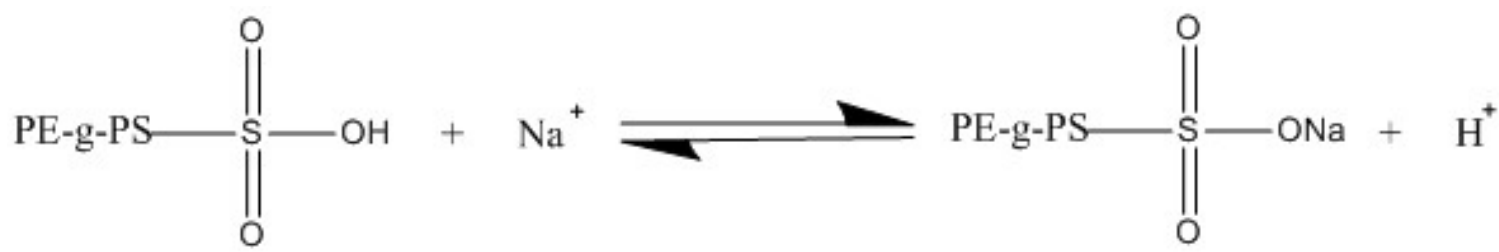

Scheme 3.3. Ion exchange with sodium cation in solution

The IEC of the anion exchange membranes were determined by analytically titrating the $\mathrm{Cl}^{-}$in anion exchange membranes of $\mathrm{Cl}^{-}$form using $0.01 \mathrm{~mol} \cdot \mathrm{L}^{-1} \mathrm{AgNO}_{3}$ solution as the titrant and $2^{\prime}, 7^{\prime}$-dichlorofluorescein as the indicator using the equation (2)

$\operatorname{IEC}\left(\mathrm{mmol} \cdot \mathrm{g}^{-1}\right)=\left(\mathrm{V}_{\mathrm{AgNO}_{3}} \times \mathrm{C}_{\mathrm{AgNO}}\right) / \mathrm{W}_{\mathrm{dry}}$

where $\mathrm{C}$ is the concentration of $\mathrm{AgNO}_{3}, \mathrm{~V}$ is the volume of $\mathrm{AgNO}_{3}$ used in the titration and $\mathrm{W}_{\mathrm{dry}}$ is the mass of dried membrane. 


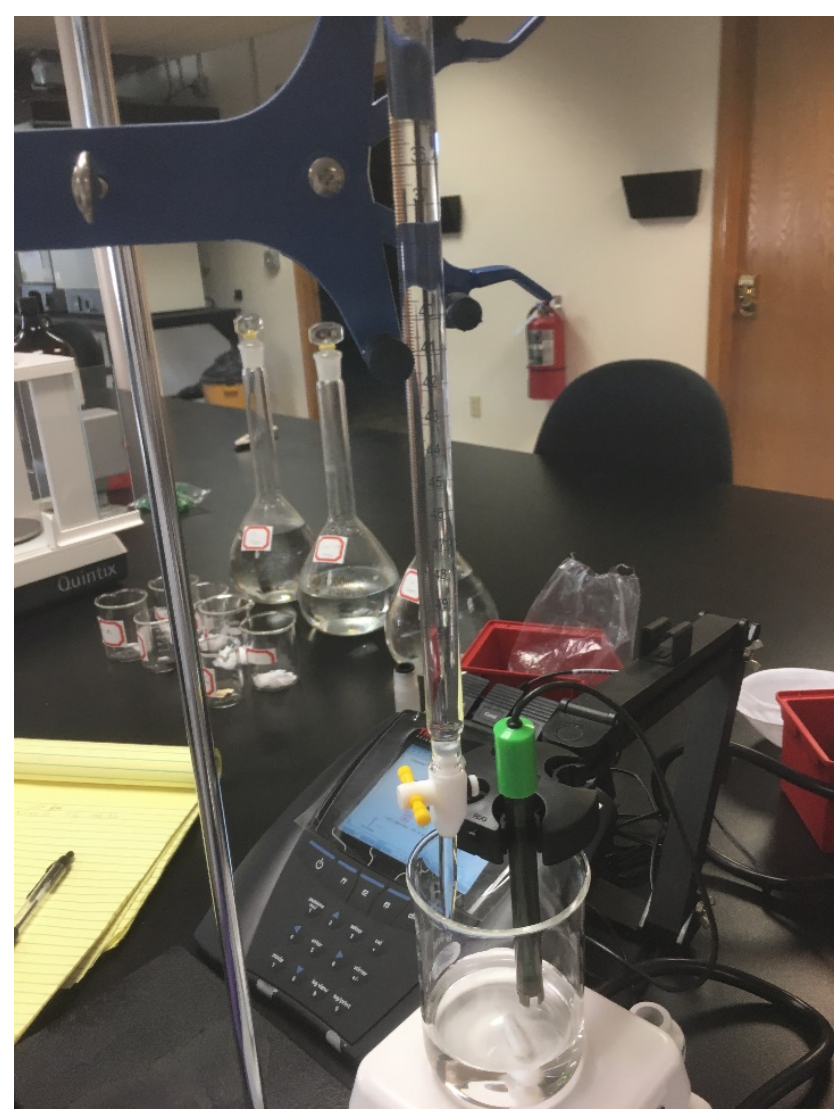

Figure 3.2. Titration instrument

\subsubsection{Water uptake and Swelling rate}

The water uptake experiments were conducted by measuring the weight differences between dried membranes and fully hydrated membranes. The membranes were cut into small pieces and immerse into deionized water at $25{ }^{\circ} \mathrm{C}$ with designed duration. After that, the surface moisture was removed, and mass was weighed $\left(\mathrm{W}_{\text {wet }}\right)$. The wet membrane was dried until the water evaporates completely and weighed again ( $\left.\mathrm{W}_{\text {dry }}\right)$. The water uptake can be determined by formula (3)

$$
\text { Water uptake }=\left(\mathrm{W}_{\text {wet }}-\mathrm{W}_{\text {dry }}\right) / \mathrm{W}_{\text {dry }} \times 100 \%
$$

The extent of swelling in membrane composite can be determined via the changes in linear dimensions of edge length. It can be calculated using the formula (4):

$$
\text { Swelling rate }=\frac{L_{S}-L_{O}}{L_{o}} \times 100 \%
$$


Where, Ls is the mean value of swollen membrane edge length, the Lo mean value of original edge length.

\subsubsection{Membrane conductivity and activation energy measurements}

Conductivity of fully hydrated membranes were measured using a potentiostat/galvanostat EIS analyzer (AMETEK, model PARSTAT 4000) (Figure 3.3). The membranes were sandwiched between two in-house made electrochemical cell parts ([Figure 3.4.) after equilibrating membrane in deionized water for $24 \mathrm{~h}$. The sinusoidal alternating currents (AC) were supplied to the electrodes for recording the frequency at a scanning rate of $1 \mu \mathrm{A} \cdot \mathrm{s}^{-1}$ within a frequency range of $10^{6}$ to $1 \mathrm{~Hz}$. The membrane resistance was determined from Nyquist plots [123]. The proton conductivity $\left(\kappa^{\mathrm{m}}\right)$ was calculated from eq (4) [123]:

$\kappa^{m}\left(\frac{s}{c m}\right)=\frac{L(\mathrm{~cm})}{\left(R(\Omega) \times A\left(\mathrm{~cm}^{2}\right)\right.}$

where, $\mathrm{L}$ is the distance between the electrodes used to measure the potential, $\mathrm{R}$ is the resistance of the membrane, and $\mathrm{A}$ is the surface area of the membrane.

Activation energy $(\mathrm{Ea})$ of membrane was estimated by plotting the graph between $\ln \kappa^{\mathrm{m}}$ $\left(\mathrm{S} \cdot \mathrm{cm}^{-1}\right)$ vs $1000 \mathrm{~T}^{-1}\left(\mathrm{~K}^{-1}\right)$ using equation (5)

$E_{a}=-b \times R$

where $b$ is the slope of the regression line and $\mathrm{R}$ is the gas constant $\left(8.314 \mathrm{~J} \cdot \mathrm{K}^{-1} \cdot \mathrm{mol}^{-1}\right)$. 


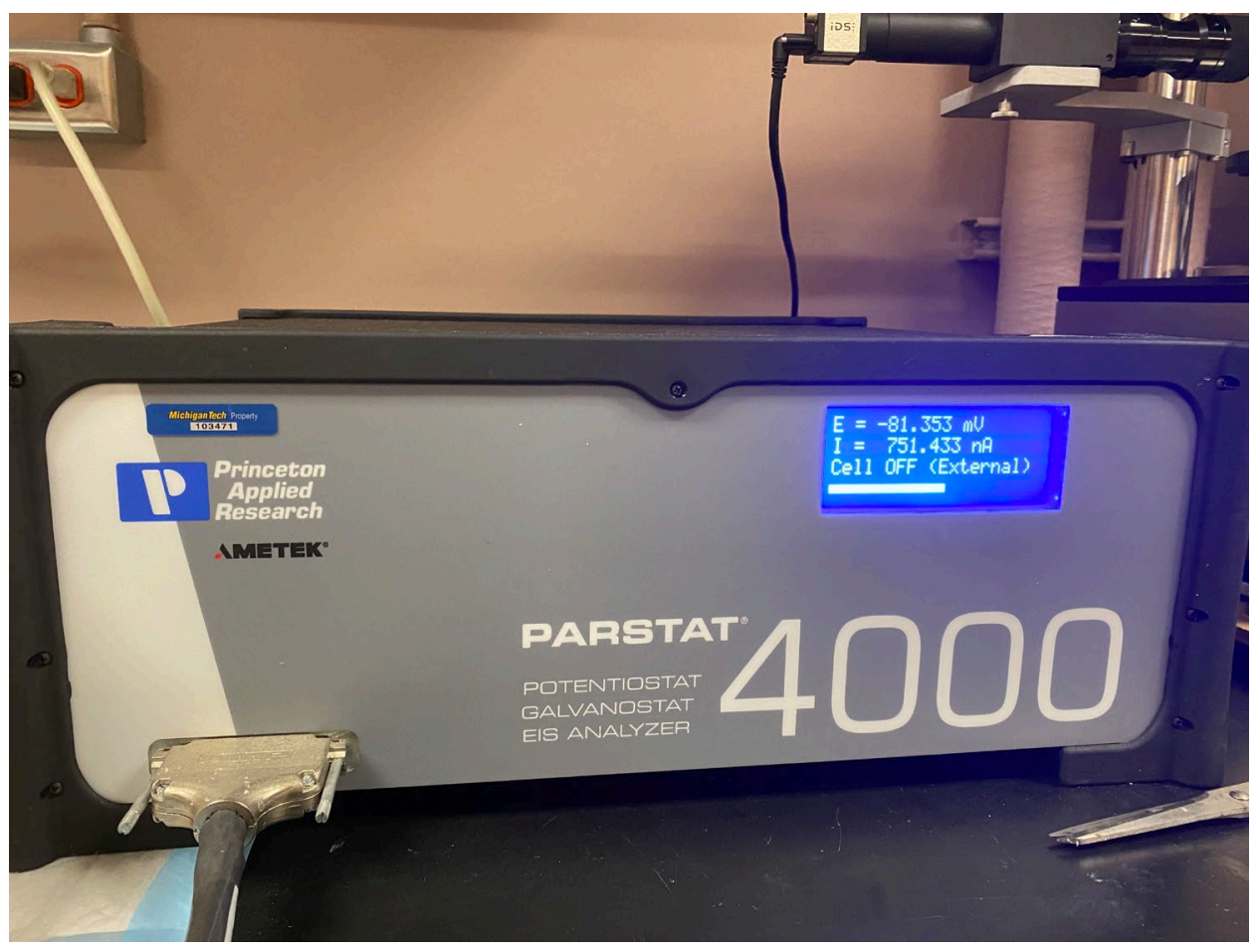

Figure 3.3. PARSTAT 4000 potentiostat/galvanostat EIS analyzer 


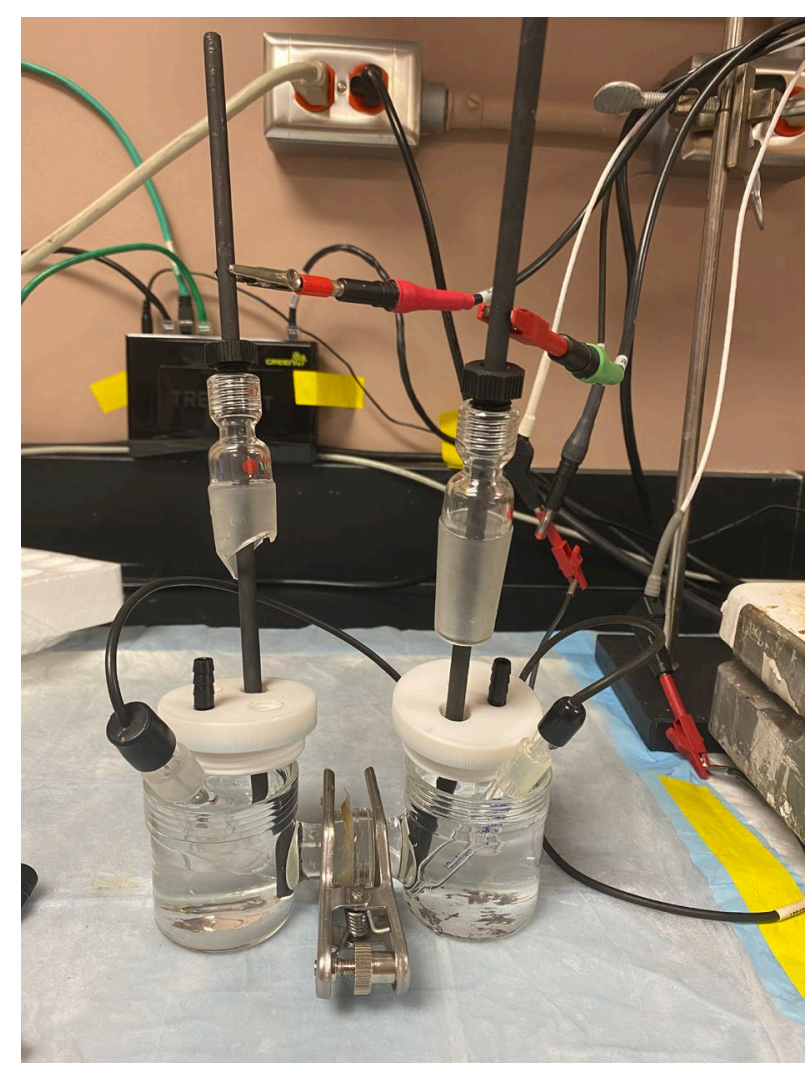

Figure 3.4. electrochemical cell parts

\subsubsection{Electrochemical analysis and electrodialysis cell evaluation}

The electrochemical analysis can be accomplished from electrochemical impedance spectroscopy (Nyquist plots) by using potentiostat/galvanostat EIS analyzer. Electrodialysis cell working performance was evaluated through running lab scale electrodialysis machine in specific condition. Typically, 7 pairs of cation and anion exchange membranes were sandwiched between anode and cathode, separated by plastic grid. Then the concentrated and diluted container were filled with $0.5 \mathrm{M} \mathrm{NaCl}$ solution. Before running, set the initial amperage as $8 \mathrm{~A}$ and record the initial voltage. After running, record the voltage value and conductivity of both concentrated and diluted solution for every $30 \mathrm{~min}$ until the conductivity is stable. The amperage value should be kept invariable during the testing. 


\section{Investigation of polyolefin-based cation exchange membrane}

\subsection{Introduction}

The basic idea of preparing cation exchange membranes in this research is grafting monomers on inert polymer to synthesize copolymer followed by introducing anionic groups on it. Usually, the cation exchange membranes are functionalized through sulfonation to attach the $\mathrm{SO}_{3} \mathrm{H}^{-}$on the copolymer. The ion exchange capacity, membrane conductivity, mechanical property and thermal stability were investigated to optimize the synthesizing conditions. Based on above concerned properties, three aspects were explored improve the properties of current cation exchange membrane and the relative reaction mechanism were invested. These three aspects include studying the effect of the inert polymer type on membrane properties, the component ratio among monomers, initiator and crosslinked agent, as well as the reaction conditions. The research started from using low density polyethylene as inert polymer to synthesize cation exchange membrane. The high-density polyethylene, linear low-density polyethylene, polypropylene and polyvinylchloride were also alternative inert polymers to be studied. The optimized inert polymer was chosen by studying the effect of chain architecture on ion exchange capacity and mechanical properties. On the other hand, polystyrene as a widely used active polymer precursor, was grafted on the inert polymer through the chemical induced polymerization and further functionalized by sulfonation. During the synthetic process, with the presence of initiator which can attack polyethylene, the monomer can also be triggered by initiator to be grated on inert polymer. The relations between components and ion exchange capacity, water uptake property and dimension stability were quantitively analyzed. For the synthesizing conditions, the effects of reaction temperature and duration of solfonaiton on the various properties of cation exchange membranes were also be assessed.

\subsection{Synthesizing mechanism}

To prepare cation exchange membranes, introducing anionic groups into inert polymer matrix is an essential path to make membrane conductive and permselective. The primary process of introducing ionic groups is grafting monomers on inert polymer to form copolymer which can connect ionic groups. Polyolefin (such as polyethylene) was chosen to be the inert polymer, the styrene was the primary monomers to be grafted. But neither polyethylene nor styrene have radical sites in their structures, which may directly combine to form a copolymer. To solve this issue, the chemical initiator was introduced. With the appearance of the initiator, the polyethylene can be triggered to form activated sites and be able to copolymerize with polystyrene. Take the initiator benzoyl peroxide (BPO) as an example. One BPO initiator can generate two benzoyl peroxide radicals. 
These radicals can attack polyethylene molecule chains and triggers a copolymerization to combine styrene. During the reaction, the divinylbenzene peroxide (DVB) was introduced as the crosslinked agents. After sulfonation of PE-g-PS copolymer in dichloroethane with chlorosulfonic, the ionic group can be added on the copolymer. The typical synthesis scheme is illustrated in Scheme 4.1.

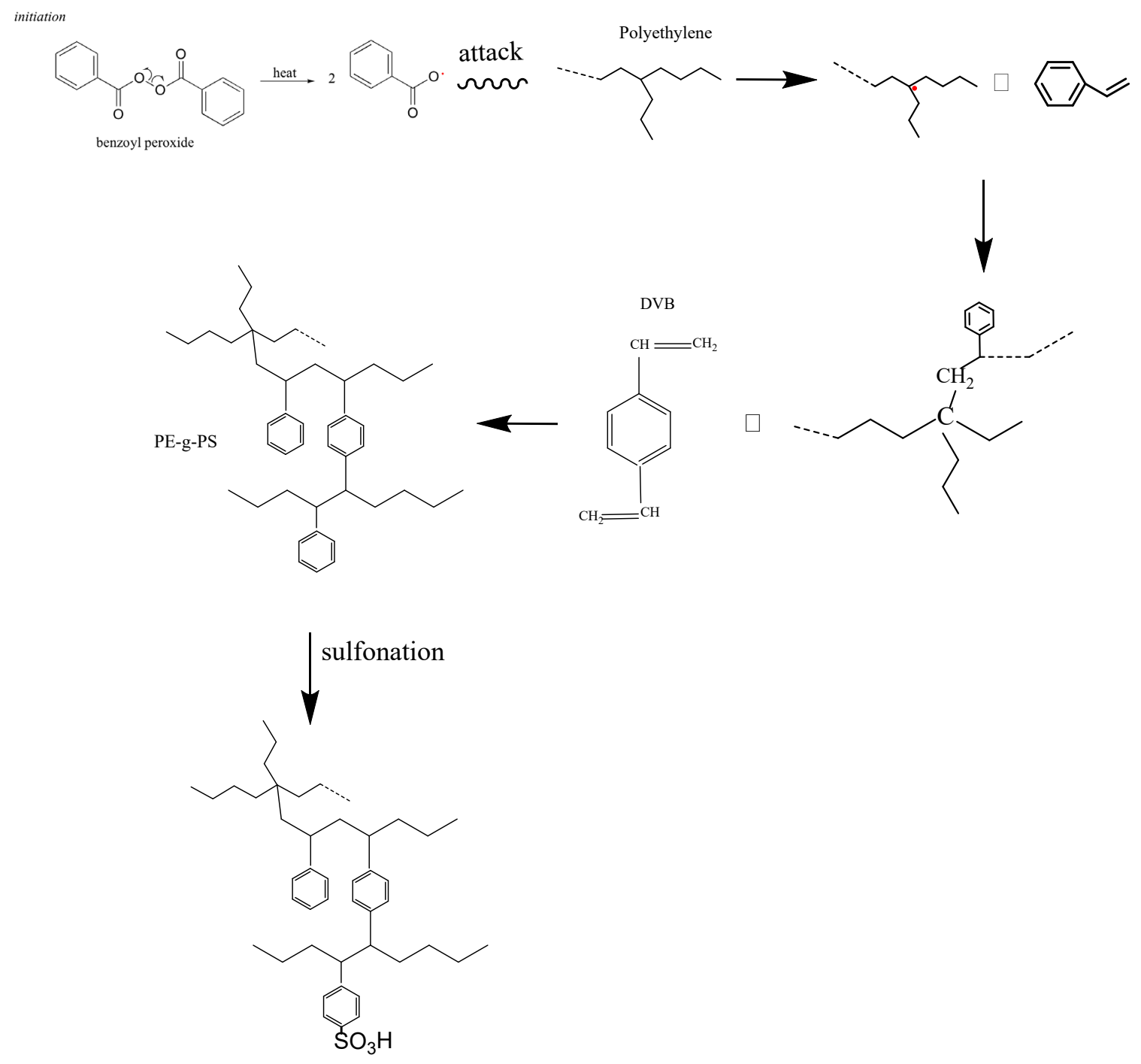

Scheme 4.1. Synthesis scheme of PE-g-PS and sulfonated PE-g-PS 


\subsection{Experimental results and discussion}

\subsubsection{The effect of inert polymer type on cation exchange membrane properties}

Based on the above discussion, the mechanical property, structure characteristic, and crystallinity of inert polymer are key factors of affecting the related properties of final membranes. Polyethylene as the most widely used commercial plastic material, has the properties of good mechanical property and excellent chemical stability. The low-density polyethylene (LDPE), high density polyethylene (HDPE) and linear lowdensity polyethylene (LLDPE) are the three most common polyethylene types. They were studied the effects of different chain architectures and crystallinity on the properties of ion exchange membranes. Besides the polyethylene, the polypropylene and polyvinyl chloride were also considered as the alternative inert polymer. The preparation of cation exchange membrane with various inert polymer followed the general synthesis procedure elaborated in section 3.2.1.1 and 3.2.1.2. The mass ratio between inert polymer (such as PE) and styrene was 1:1, the DVB and benzoyl peroxide were added in at a weight ratio of $3 \%$ and $0.5 \%$ to styrene respectively.

\subsubsection{The effect of polyethylene structure on ion exchange capacity}

The appearances of sulfonated cation exchange membrane with different inert polymer were presented in Figure 4.1. After sulfonation, the LDPE-g-PS based membrane presents dark brown color, the LLDPE-g-PS membrane is yellow brown, while the HDPE-g-PS membrane is pale brown with white part. The Figure 4.1. indicates the LDPE-g-PS copolymer accepted the largest amount of $\mathrm{SO}_{3} \mathrm{H}^{-}$and was oxidized thoroughly by the sulfonation. The LLDPE-g-PS copolymer has the moderate ability to accept $\mathrm{SO}_{3} \mathrm{H}^{-}$. The HDPE-g-PS copolymer attracted the lowest amount of $\mathrm{SO}_{3} \mathrm{H}^{-}$, making part of the inert polymer keep original color due to sulfonation failure.

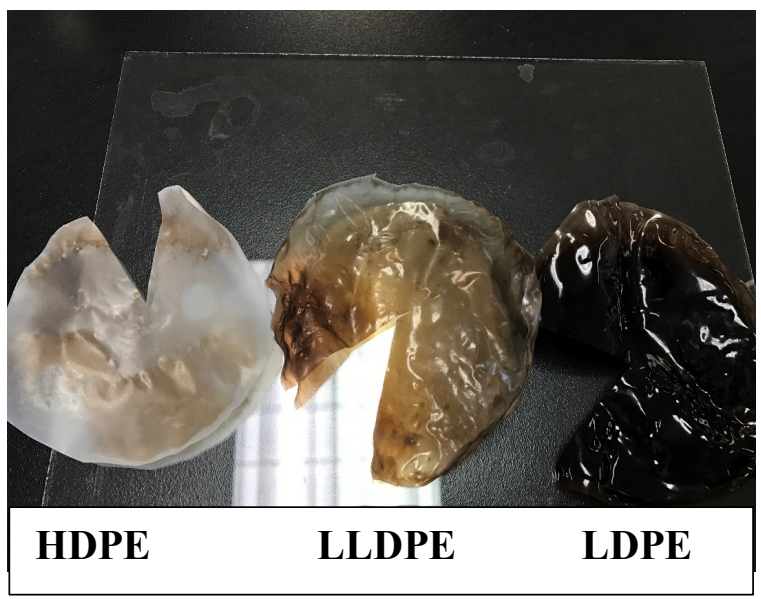


Figure 4.1. Sulfonated cation exchange membrane with different inert polymer

Two methods were conducted to test the IEC value of cation exchange membrane with different inert polymer (based material), including elemental analysis and titration test. The sulfonic group $\mathrm{HSO}_{3}{ }^{-}$has the same mole numbers of $\mathrm{S}^{2-}$ and $\mathrm{H}^{+}$. The mole numbers of $\mathrm{HSO}_{3}{ }^{-}$can be obtained through measuring the mole numbers of $\mathrm{S}^{2-}$ by using elemental analysis and titration test. The IEC value of $\mathrm{H}^{+}$can also be calculated by these results. The elemental analysis results (Table 4.1. 4.2. and 4.3.) shows the $\mathrm{C}, \mathrm{H}, \mathrm{N}, \mathrm{S}$ content of original PE, PE-g-PS copolymer and sulfonated PE-g-PS. The increasing of S of final $\mathrm{CEM}$ attributed to the inert polymer absorbed $\mathrm{HSO}_{3}{ }^{-}$through sulfonation and regard as the enhancement of functional groups. The sulfonated LDPE-g-PS membrane obtain the highest IEC value $(1.78 \mathrm{mmol} / \mathrm{g})$, which is $18.7 \%$ and $747 \%$ higher than the LLDPE-g-PS based membrane and HDPE-g-PS based membrane respectively. The IEC of sulfonated LDPE-g-PS membrane is superior to the most commercial cation exchange membrane in Table 1.1.

The IEC data in Figure 4.2. were tested from the titration method and presents the same trend as Table 4.1-4.3. The sulfonated HDPE-PS membranes have the lowest IEC value, $85 \%$ lower than the sulfonated LDPE-PS membranes and $82 \%$ lower than the sulfonated LLDPE-PS membranes. The reason of causing such results is related on the structure of the different types of based membrane.

Table 4.1. The elemental analysis results of LDPE, LDPE-g-PS and Sulfonated LDPE-gPS copolymer

\begin{tabular}{|c|c|c|c|c|}
\hline Element & $\mathbf{C}$ & $\mathbf{H}$ & $\mathbf{N}$ & $\mathbf{S}$ \\
\hline LDPE & $85.13 \%$ & $12.47 \%$ & $0.39 \%$ & $2.59 \%$ \\
\hline LDPE-g-PS & $87.61 \%$ & $12.67 \%$ & $0.56 \%$ & $2.35 \%$ \\
\hline $\begin{array}{l}\text { Sulfonated } \\
\text { LDPE-g-PS }\end{array}$ & $67.35 \%$ & $10.62 \%$ & $0.61 \%$ & $8.35 \%$ \\
\hline Weight & \multicolumn{4}{|c|}{$0.648 \mathrm{~g}$} \\
\hline $\begin{array}{l}\text { Ion exchanged } \\
\text { capacity }\end{array}$ & & \multicolumn{3}{|c|}{$\begin{array}{c}\frac{(\text { sulfnated LDPE. PS S } \%-\text { LDPE S\%) } \times \text { Weight }}{\text { Mole weight of S(32) } \times \text { Weight }} \times 1000 \\
=1.78 \mathrm{mmol} / \mathrm{g}\end{array}$} \\
\hline
\end{tabular}

Table 4.2. The elemental analysis results of LLDPE, LLDPE-g-PS and Sulfonated LLDPE-g-PS copolymer

\begin{tabular}{lllll} 
Element & C & H & N & S \\
\hline
\end{tabular}




\begin{tabular}{|c|c|c|c|c|}
\hline LLDPE & $83.86 \%$ & $14.19 \%$ & $0.43 \%$ & $2.55 \%$ \\
\hline LLDPE-g-PS & $85.79 \%$ & $12.45 \%$ & $0.54 \%$ & $2.32 \%$ \\
\hline $\begin{array}{l}\text { Sulfonated } \\
\text { LLDPE-g-PS }\end{array}$ & $69.38 \%$ & $10.80 \%$ & $0.66 \%$ & $7.36 \%$ \\
\hline Weight & \multicolumn{4}{|c|}{$0.464 \mathrm{~g}$} \\
\hline $\begin{array}{l}\text { Ion exchanged } \\
\text { capacity }\end{array}$ & \multicolumn{4}{|c|}{$1.5 \mathrm{mmol} / \mathrm{g}$} \\
\hline
\end{tabular}

Table 4.3. The elemental analysis results of HDPE, HDPE-g-PS and Sulfonated HDPE-gPS copolymer

\begin{tabular}{lllll}
\hline Element & $\mathbf{C}$ & $\mathbf{H}$ & $\mathbf{N}$ & $\mathbf{S}$ \\
\hline HDPE & $78.53 \%$ & $13.39 \%$ & $10.50 \%$ & $2.78 \%$ \\
\hline HDPE-g-PS & $80.26 \%$ & $12.68 \%$ & $0.44 \%$ & $3.12 \%$ \\
\hline $\begin{array}{l}\text { Sulfonated HDPE- } \\
\text { g-PS }\end{array}$ & $79.32 \%$ & $13.34 \%$ & $0.66 \%$ & $4.12 \%$ \\
$\begin{array}{l}\text { Weight } \\
\begin{array}{l}\text { Ion exchanged } \\
\text { capacity }\end{array}\end{array}$ & & $0.502 \mathrm{~g}$ & \\
\hline
\end{tabular}




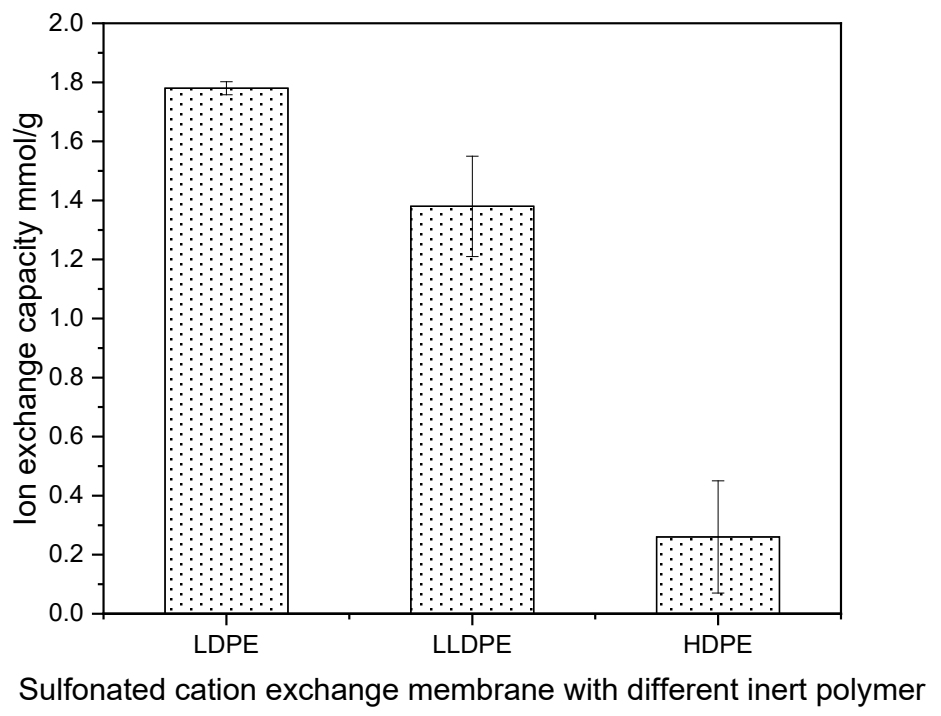

Figure 4.2. The comparison of IEC in different types of membranes

Although these three types of polyolefin have the same monomers - ethylene, they possess different molecular structures and crystallinity. LDPE is produced by the highpressure process by radical polymerization, thereby it has a high degree of short and long chain branches [92]. These high degrees of branches with long and short chains do not pack into the crystal structure as well. It has, therefore, less strong intermolecular forces as the instantaneous-dipole induced-dipole attraction is less. Unlike LDPE, HDPE is primarily a linear with minor branching, so the intermolecular forces are stronger than in LDPE which has the highly branched polymer. LLDPE is a substantially linear polymer with significant numbers of short branches, commonly made by copolymerization of ethylene with short-chain alpha-olefins. The degree of branching and crystallinity are between the LDPE and HDPE. The branching degree of these three types polyethylene is represented in Figure 4.3.

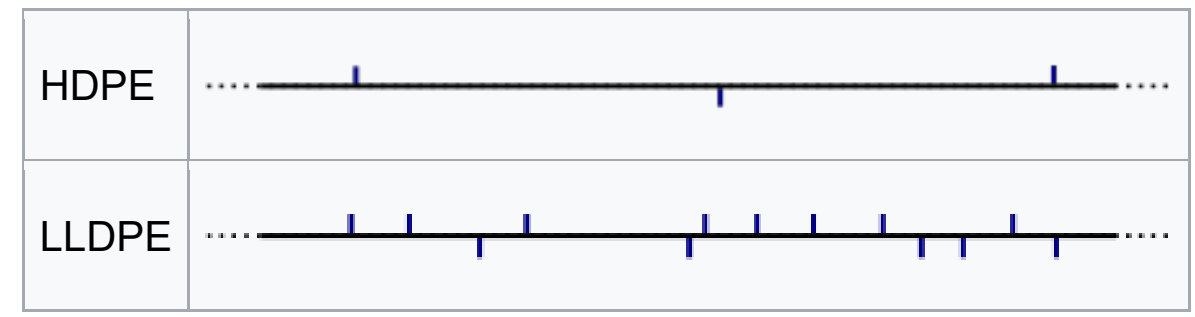




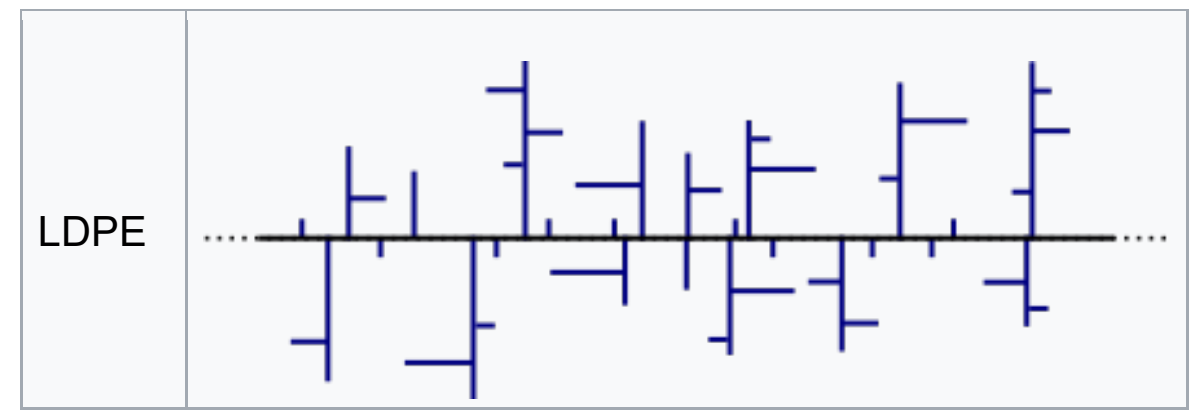

Figure 4.3. The branching degree of LDPE, LLDPE and HDPE. Image resource: https://en.wikipedia.org/wiki/Polyethylene

The incompact structure density and low degree of crystallinity provide the LDPE more space to contact initiator and obtain stimulating. This makes another monomer can be swelled into the PE structure more easily. While the HDPE and LLDPE have the higher crystallinity and compact molecular structure, so the opportunities of being attacked by the initiator are largely decreased. The LDPE have the branched structure, which means LDPE have more available sites can be grafted by polystyrene, while the HDPE have the linear structure, far lower accesses to be grafted. The LLDPE are between the middle. Furthermore, the free-radical polymerization mechanism also provides the explanation why the LDPE obtain the higher grafted degree. In the radical polymerization process of the HDPE, the initiators prefer attacking the radical sites at the ends of the growing polyethylene molecules due to the stabilization. This cause the new monomer radicals tend to add to the ends of the chain structure [37]. In the LDPE chain structure, the secondary radicals which in the middle of a chain are more stable than the primary radicals which in the end of the chain, and the tertiary radicals which at a branch point are most stable [38]. This stability comparation among these three radical sites is illustrated in Figure 4.4.

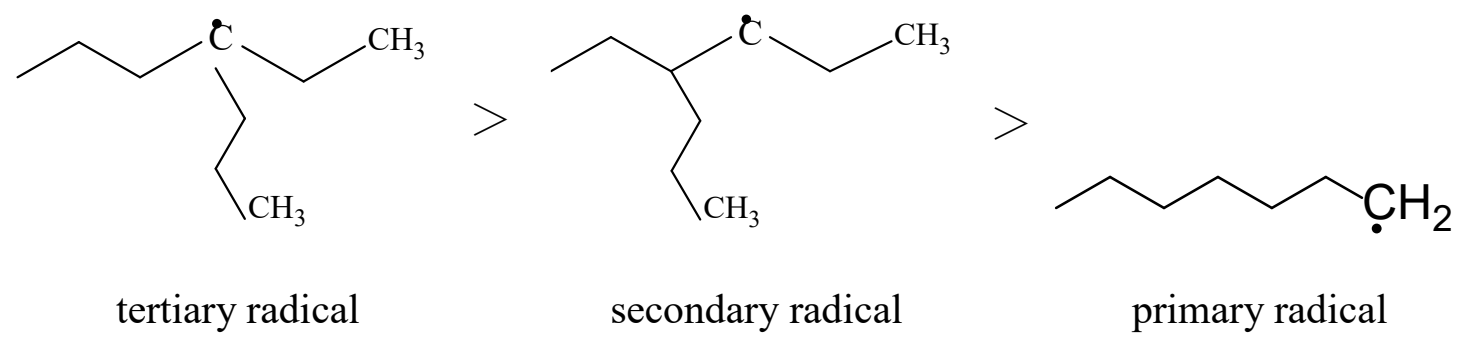

Figure 4.4. Comparison of the stability with the primary radical, secondary radical and tertiary radical in LDPE

Each time the initiator attacked the LDPE, the chain will create a primary radical, but they will be arranged to form the more stable secondary or tertiary radicals [39]. This means the free radical sites which connect the branching carbon can be attacked by the 
initiators more easily. This free radical polymerization process is illustrated in Figure 4.5. Compare to the LLDPE and HDPE, LDPE has the higher density of the long and short branching, which means it has more radical sites that can accept another monomer to be grafted. When styrene monomers are close to polystyrene, they have more opportunities to be grafted on the LDPE.

The polyethylene-polystyrene can be sulfonated by functional group $\mathrm{HSO}_{3}^{-}$, and the ion exchange capacity is related on it. Therefore, this founds the connection between ion exchange capacity and the grafted degree, further related on the chain structure of inert polymer.
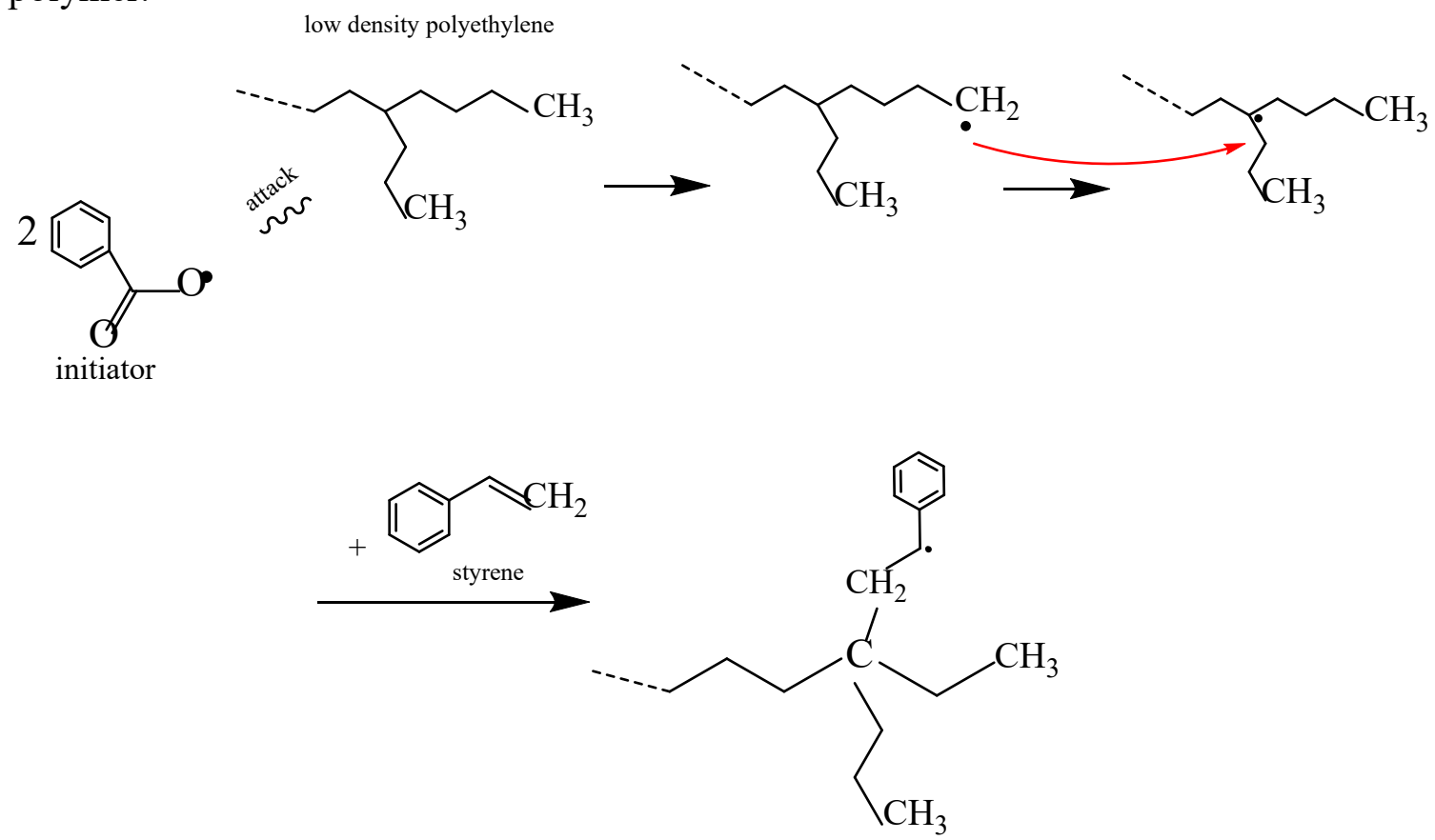

Figure 4.5. Free radical polymerization process

\subsubsection{FT-IR spectrum analysis}

FT-IR spectrum of different chain structure of PE films, their grafted copolymer and sulfonated membranes are presented in Figure 4.6, 4.7 and 4.8. The spectrums reveal the polyethylene have been grafted on LDPE, LLDPE and HDPE successfully. Compare with the initial LDPE, LLDPE and HDPE IR spectrum, there are some additional peaks, including the peaks at 3067 and $3030 \mathrm{~cm}^{-1}$ which are corresponding to the $\mathrm{C}-\mathrm{H}$ stretching on benzene ring; the peaks at 1601 and $1439 \mathrm{~cm}^{-1}$ which are corresponding to the $\mathrm{C}=\mathrm{C}$ stretching of benzene ring; the very sharp peaks at 699 and $756 \mathrm{~cm}^{-1}$ are due to the vibration of $\mathrm{C}-\mathrm{H}$ on benzene. 
The infrared spectrums of these three figures show the sulfonation reaction on the membranes are also successful. The FTIR results of these three spectrums show weak and broad bands over $3000-3500 \mathrm{~cm}^{-1}$, indicating a $\mathrm{OH}$ stretching, and intense peak just above $1000 \mathrm{~cm}^{-1}$, corresponding to a $\mathrm{S}=\mathrm{O}$ group. We suspect under this condition, the LDPE-g-PS, LLDPE-g-PS and HDPE-g-PS copolymers were partially overoxidized by concentrated sulfuric acid since new peaks appearing at around 1160 and $1650 \mathrm{~cm}^{-1}$, corresponding the $\mathrm{C}-\mathrm{O}$ and $\mathrm{C}=\mathrm{O}$ bonds, respectively.

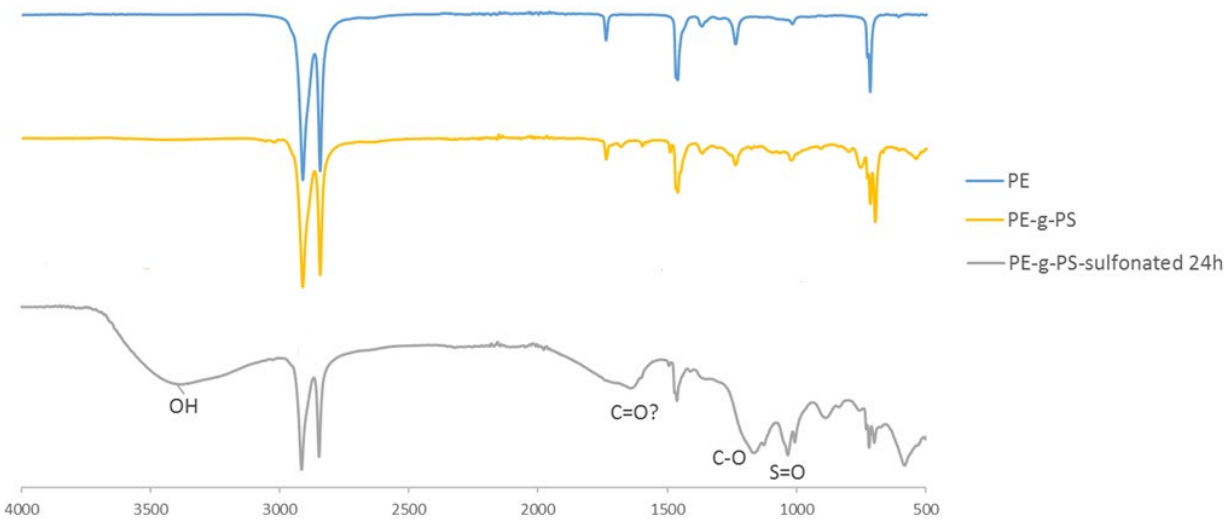

Figure 4.6. FTIR spectrum of initial LDPE, LDPE-g-PS and LDPE sulfonated membranes

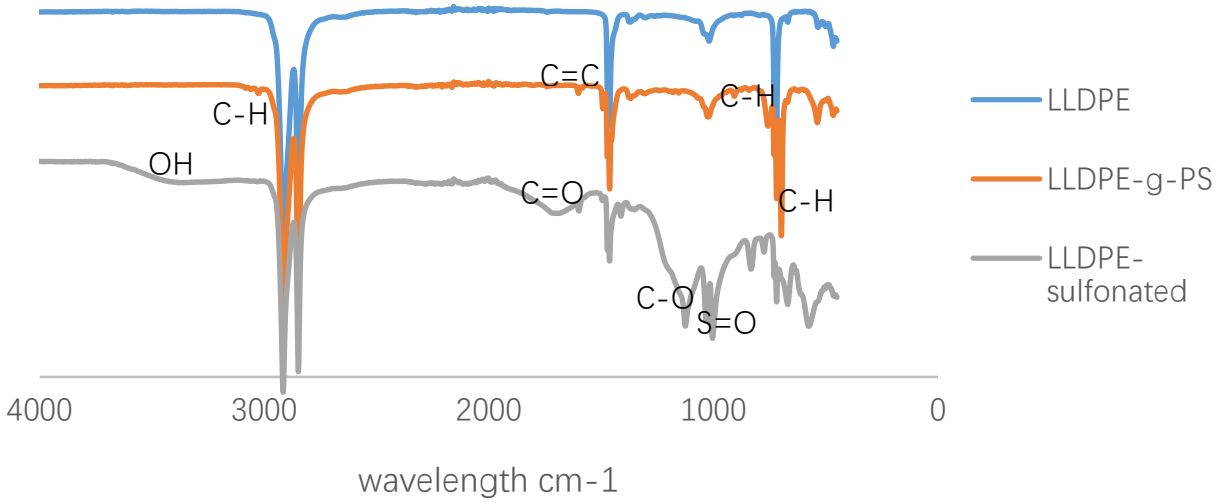

Figure 4.7. FTIR spectrum of initial LLDPE, LLDPE-g-PS and LLDPE sulfonated membranes 


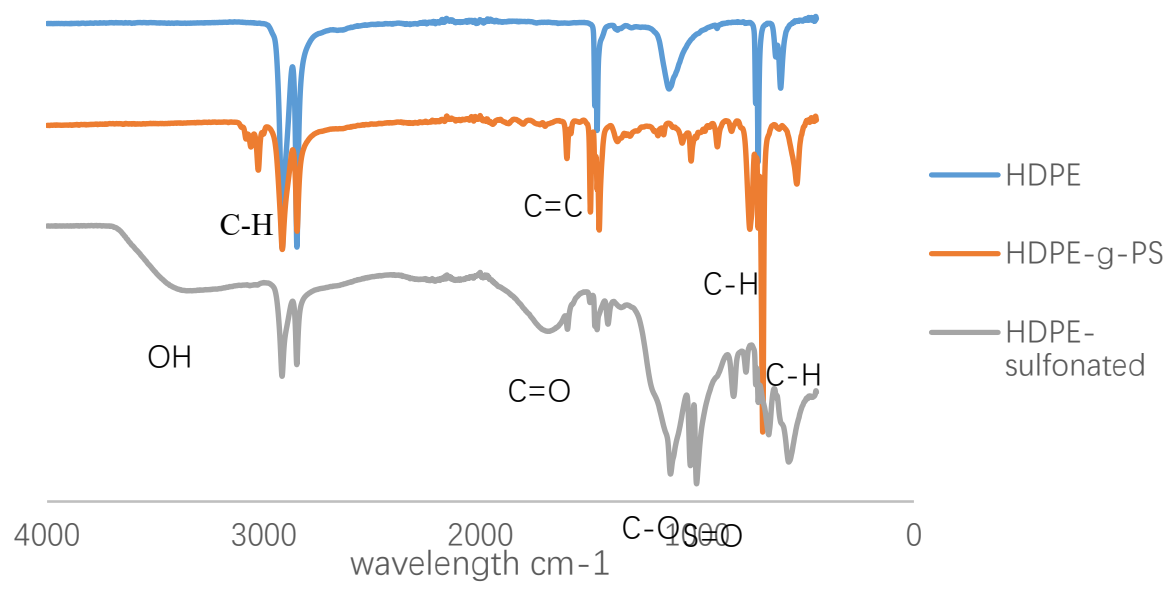

Figure 4.8. FTIR spectrum of initial HDPE, HDPE-g-PS and HDPE sulfonated membranes

To compare the grafted degree of polystyrene on polyethylene chains, the infrared spectrums of LDPE-g-PS, LLDPE-g-PS and HDPE-g-PS were picked up and combined (Figure 4.9.). Compare to the LDPE-g-PS infrared spectrum, the LLDPE-g-PS and HDPE-g-PS have the weaker sharp of additional peaks $(\mathrm{C}-\mathrm{H}$ and $\mathrm{C}=\mathrm{C}$ peaks $)$, which reflects the less polystyrene grafted on the initial membrane. This result accord with the IEC testing.

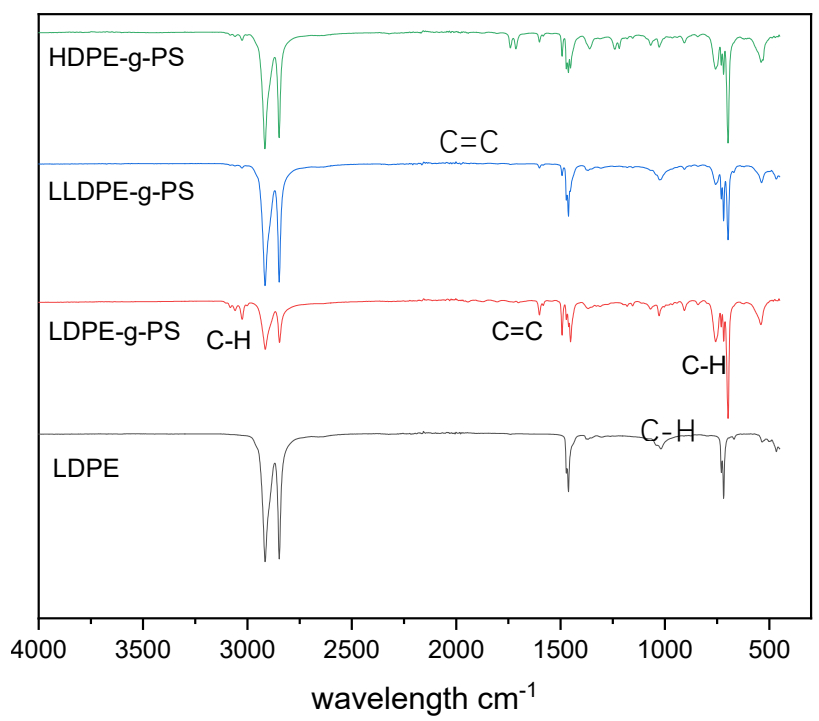

Figure 4.9. Infrared spectrum of PE-g-PS membranes with the different based membranes 


\subsubsection{The effect of other inert polymer types on membrane properties}

\subsection{Physical properties}

Besides polyolefin-based polymer, other commercial polymers were also considered as the inert polymer in this research due to their excellent mechanical property and thermal resistance, such as polypropylene and Polyvinyl chloride (PVC).

Polypropylene is in many aspects like polyethylene, especially in solution behavior and electrical properties. The additionally present methyl group improves mechanical properties and thermal resistance. Therefore, polypropylene is a good partly replacement of PE in membrane. However, the addition of polypropylene (PP) individually lead the increasing brittle of material, so proportion of addition need to be further studied. We mixed $40 \% \mathrm{PP}$ with $\mathrm{PE}$, then treated them as an inert polymer to react with styrene (St). The reaction compositions include PP 2g, PE 5g, St 7.5g, DVB 0.225g, BPO $0.01 \mathrm{~g}$ respectively. There are two things need to be paid attention, the PP can be easier solved in xylene, the proportion of xylene in the solvent for PP/PE mixture should be predominate; another thing is the melting temperature of $\mathrm{PP} / \mathrm{PE}$ mixture is higher than the pure PE. The sulfonating procedure is the same as PE-g-PS sulfonation.

$\mathrm{PVC}$ is the world's third-most widely produced synthetic plastic polymer, after polyethylene and polypropylene. There are chlorides on alternating carbon centers in the PVC linear structure. Compare to the PE, the PVC has high hardness and good mechanical properties. It supposed to be a good choice for preparing membrane. Firstly, solubility of PVC was invested by using various solvent, such as toluene, THF and ethanol. It was proved the THF has the highest solubility to PVC at the elevating temperature. Then use the same styrene and initiator proportions to prepare PVC-g-PS membranes, followed by the sulfonation process.

A comparation of mechanical properties, water uptake and ion exchange among PE-g-PS CEM, PP-g-PS CEM, PVC-g-PS CEM are listed in table 4.4. The mechanical properties (burst strength and tensile strength) enhanced when PP and PVC replace the PE as the inert polymer respectively. The extension properties of PVC-g-PS CEM is not as good as PE-g-PS CEM due to the PVC-g-PS membranes lost the toughness and became brittle. It is suspected that the plasticizer included in the PVC has been abstracted by the solvent. These results indicated that the PP replacement make the CEM become strong and tough. But PVC replacement only increase strength, having no contribution to toughness enhancement. Compare to the PE-g-PS CEM, the ion exchange and conductive properties became weaker for PP-g-PS and PVC-g-PS CEM.

Table 4.4. Physical properties of three kinds of CEM with different based materials

PE-g-PS CEM PP-g-PS CEM PVC-g-PS CEM




\begin{tabular}{lccc}
\hline $\begin{array}{c}\text { Burst strength/thickness } \\
\left(\mathrm{kpa} \cdot \mathrm{mm}^{-1}\right)\end{array}$ & 1890 & 2679 & 2570 \\
\hline Tensile strength(Y)/MPa & 7.2 & 11.45 & 14.2 \\
\hline Extension/mm & 12.381 & 18 & 9.643 \\
\hline Water uptake $/ \%$ & 25.7 & 19.6 & 17.3 \\
\hline IEC/ $\mathrm{mmol} \cdot \mathrm{g}^{-1}$ & 1.72 & 1.43 & 1.44 \\
\hline
\end{tabular}

\subsection{Thermal expansion property}

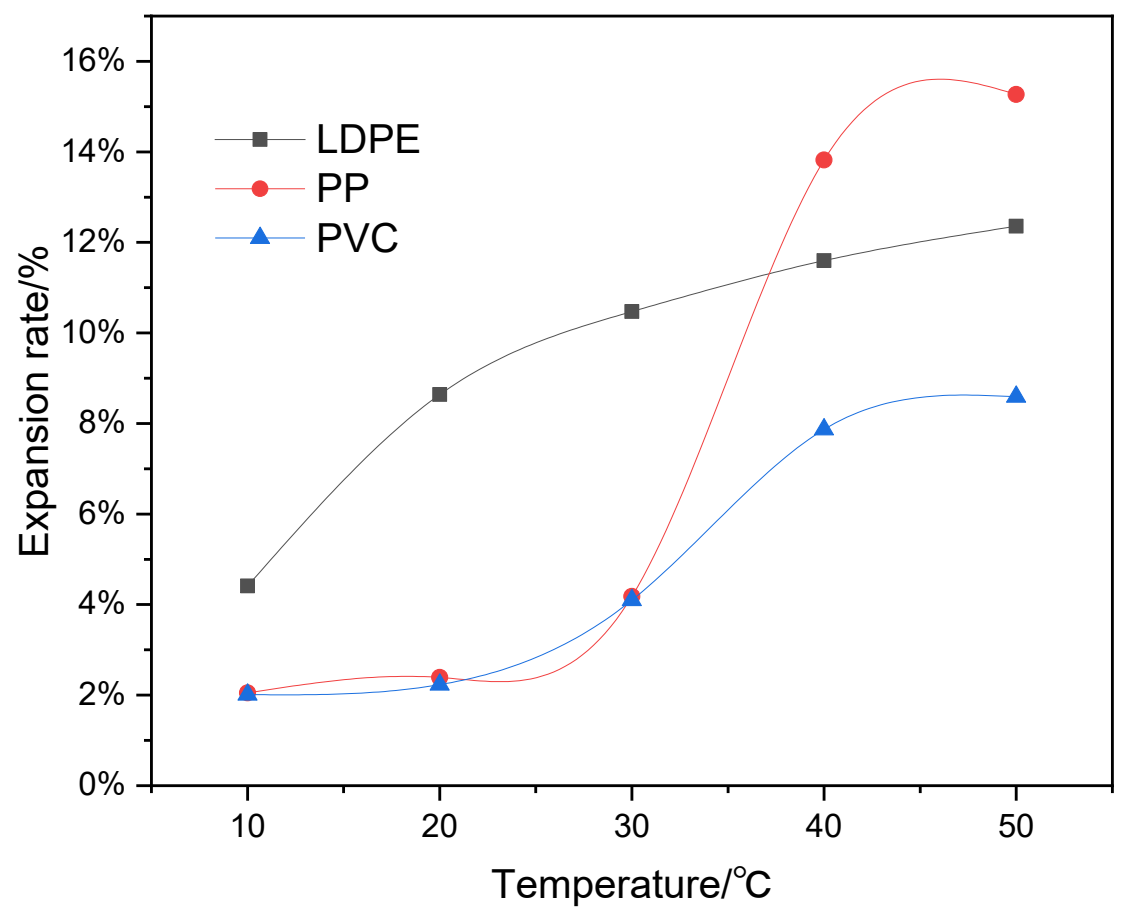

Figure 4.10. Temperature effect on the expansion rate of LDPE, PP and PVC based CEM

With increasing of temperature, the thermal expansion rate of these three based polymer types CEM increased. The expansion rate of PE based CEM increased gently. For PP and PVC based CEM, the membranes do not expand quickly when temperature below $30^{\circ} \mathrm{C}$. While the temperature goes up to $30^{\circ} \mathrm{C}-40^{\circ} \mathrm{C}$, the expansion rate of PP based CEM elevate sharply, after $40^{\circ} \mathrm{C}$, the increment tendency become gently. Compare to the PE based CEM, the PP and PVC based CEM have lower thermal expansion rate when 
temperature below $40^{\circ} \mathrm{C}$. This higher expansion rate is suspected to relate on the lower dense and loose surface structure of PE.

\subsection{Morphology characterization}

The surface morphology of PE, PP and PVC based CEM are shown in Figure 4.11. 4.12. and 4.13 respectively. The matrix surfaces of all samples have homogeneous texture. But compare with the LDPE based CEM, the PP and PVC based CEM have denser surface structure. The spheres distributed on the LDPE and PP based CEM have gel morphology and embedded firmly in the matrix material. These spheres were deduced to be sulfonated polystyrene gels through EDS analysis. The Figure 4.13 shows the distributing density of small spheres in PVC based CEM is relatively lower. Furthermore, the small photo embedded in Figure 4.13. shows the interface between spheres and matrix is very clear, indicating the interfacial adhesion between PS and PVC is poor. The lower polystyrene spheres density indicates the lower $\mathrm{SO}_{3}{ }^{2-}$ groups attached polystyrene chains. Therefore, the IEC value of PVC CEM would not be higher than other two CEMs.
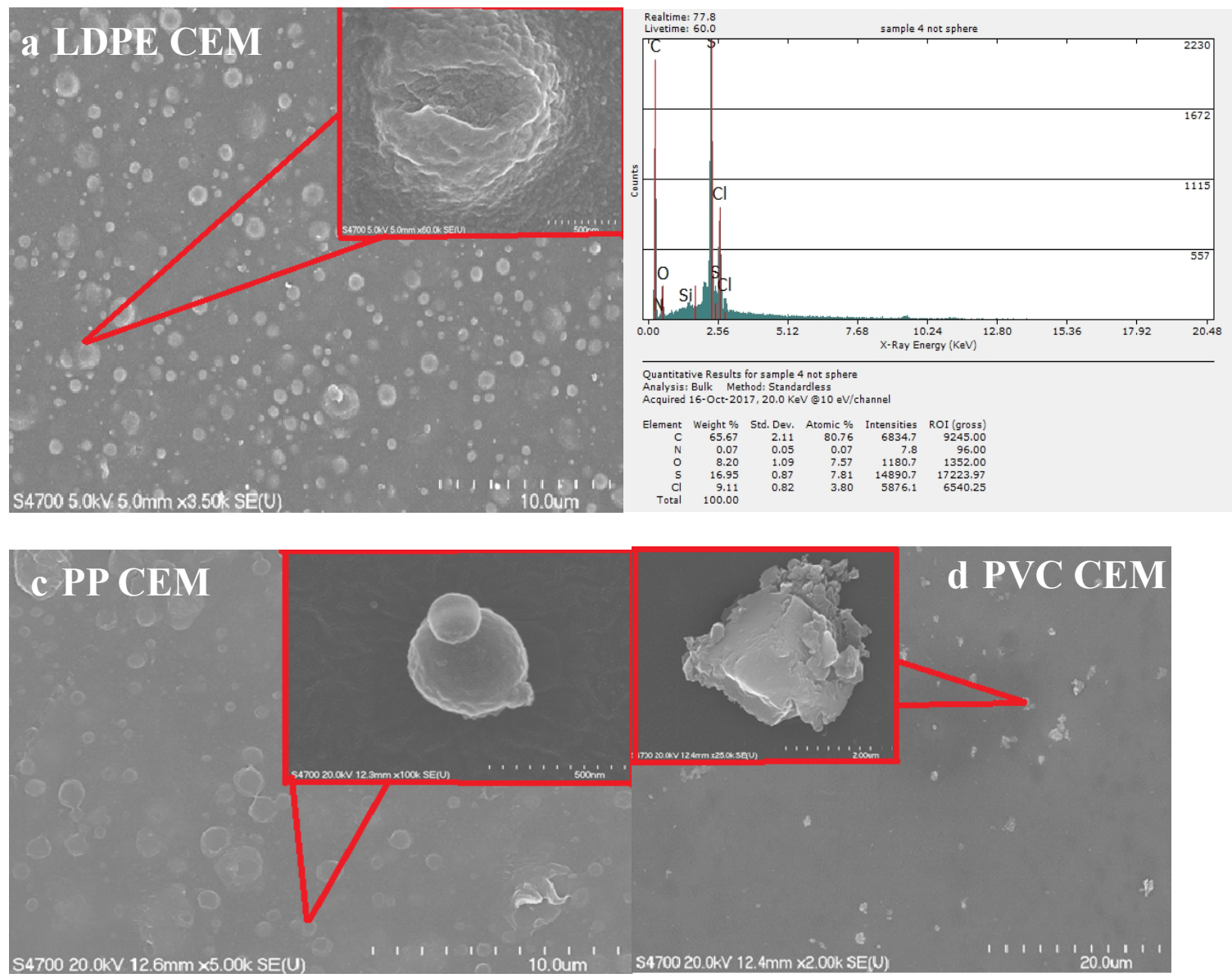
Figure 4.11. (a) SEM of LDPE based CEM (b) DES analysis of LDPE based CEM (c) SEM of PP based CEM (d) SEM of PVC based CEM

\subsubsection{The effect of styrene addition on cation exchange membrane properties}

On the process of synthesizing copolymer, based on the chemical polymerization mechanism, many factors can affect the properties of final membrane, such as the monomer ratio, initiator and crosslinked agents, reaction parameters like temperature and duration. Polymerizing the polyethylene and styrene is an effective way to synthesize cation exchange membrane. Based on this, the amounts of styrene would be the critical factor affecting the cation exchange membrane properties.

The ratios between styrene and LDPE in cation exchange membrane were varied to explore the influence of styrene addition. The investigating ratios include $0.5: 1$, 1:1,1.5:1,2:1,2.5:1 and 3:1. The crosslink agents DVB addition changed with the styrene content, keeping the $3 \%$ of styrene. The initiator BPO were added into those 6 reactions with the same amounts.

\subsubsection{The effect of styrene addition on ion exchange capacity}

The result presented in Figure 4.10. shows that as the IEC of the cation exchange membrane increase with the styrene addition increasing. The IEC value increased sharply when the ratio of styrene lower than 1.5 but goes flat when the ratios of styrene higher than 1.5. It was expected the higher addition of styrene in PE-g-PS copolymer, the higher functional groups can be attached on it. But the result did not show this trend. 


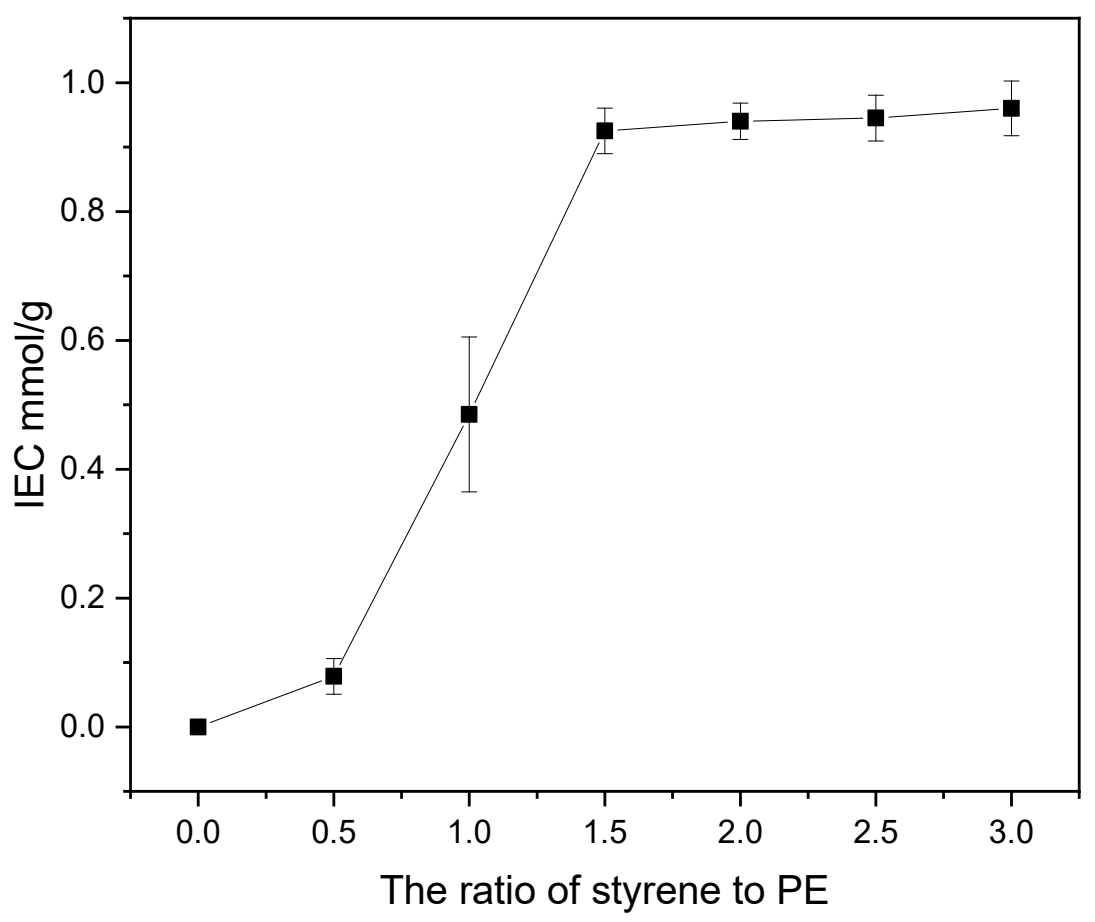

Figure 4.10. IEC value of sulfonated PE-g-PS with different styrene addition

This trend can be explained that in the PE-g-PS polymerization process, neither polyethylene nor styrene have radical sites in their structures. However, with the appearance of the initiator which can attack both polyethylene and styrene, they can form activated sites respectively. Take the common initiator benzoyl peroxide (BPO) as an example. One BPO initiator can generate two benzoyl peroxide radicals (Figure 4.11.). These radicals can either attack PE molecules or styrene monomers. It triggers a copolymerization of styrene on PE if attacking PE. Otherwise, a self-polymerization of styrene is more likely to occur. In general, the preparation of PE-g-PS copolymer by triggering of PE films in the presence of styrene monomers produce considerable amounts of self-polymer polystyrene and potentially some cross-linking products. Once the self-polymerization happened, it will contend the styrene monomers with the PE-PS copolymer. This competitive relationship between PS self-polymerization and PE-g-PS copolymerization cause the less polystyrene grafted on the base PE membranes.

Compare with the styrene self-polymerization, the graft density and graft length of copolymerization were difficult to control. The difference of the reactivity ratios between polyethylene and styrene monomer and the diffusion limitation of styrene also make the graft copolymerization not easier than the styrene self-polymerization [93]. However, the self-polymerized styrene is not stable on the materials since there is no chemical bonding with PE and can be easily removed by solvent. So, it cannot bring any contributions to the subsequent ion exchange. When the addition of styrene increase, the ion exchange capacity will increase in the earlier stage. With the continually increasing, the styrene 
self-polymerization will take over the advantage and consume the additional styrene monomers. In the later stage, the ion exchange capacity does not change with the styrene due to the mounts of self-polystyrene aggregate. Therefore, there is a competition between PE-g-PS product and self-polymerized PS. Self-polymerization will be favored when the styrene concentration is high. Thus, high styrene concentration decreases the productivity of the grafted polymers.

These explanations are illustrated in Figure 4.12.

initiation<smiles>O=C(OOC(=O)c1ccccc1C(=O)[O-])c1ccccc1</smiles>

benzoyl peroxide

Figure 4.11. BPO reaction mechanism 

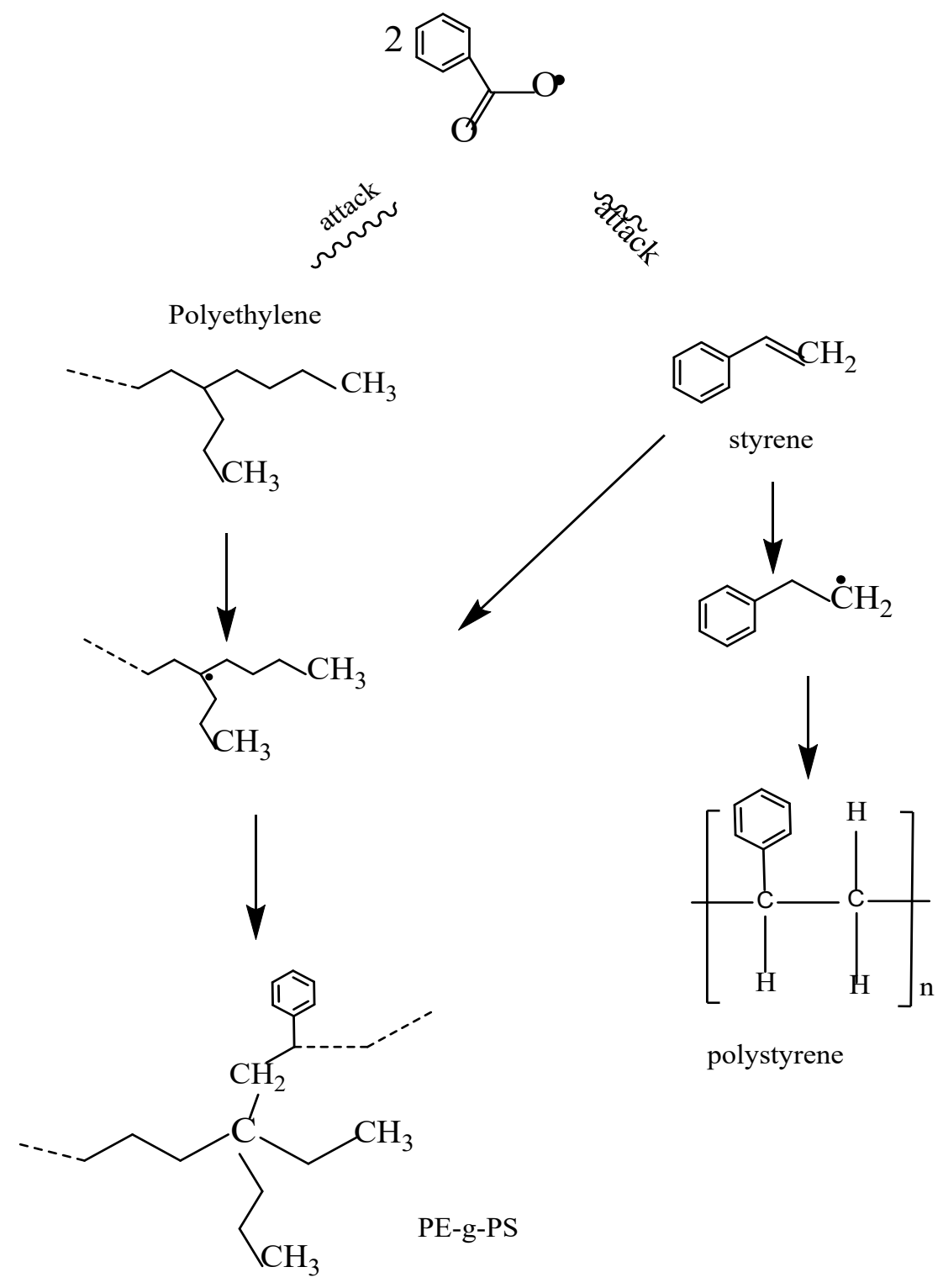

Figure 4.12. The formation mechanism of copolymer and self-polymer

When add the cross-linking agent DVB in the process of polymerization, The IEC trend can also be explained through the polymer compatibilization theory (Figure 4.13.).

Compatibilization in polymer chemistry is the addition of a substance to an immiscible blend of polymers that will increase their stability [42]. Since PE, PS exhibit very low entropy of mixing, the formation and coalescence of copolymer will result in sizable dispersed phase domains, low adhesion and poor final properties [43]. In this situation, the compatibilizer is introduced to improve adhesion, reduce interfacial tension between $\mathrm{PE} / \mathrm{PS}$ and stabilized phases through inhibiting the collision of sized droplets. Block or 
graft copolymers are commonly used as compatibilizer. When DVB was added in this system, the PS/DVB copolymer will be formed in situ.
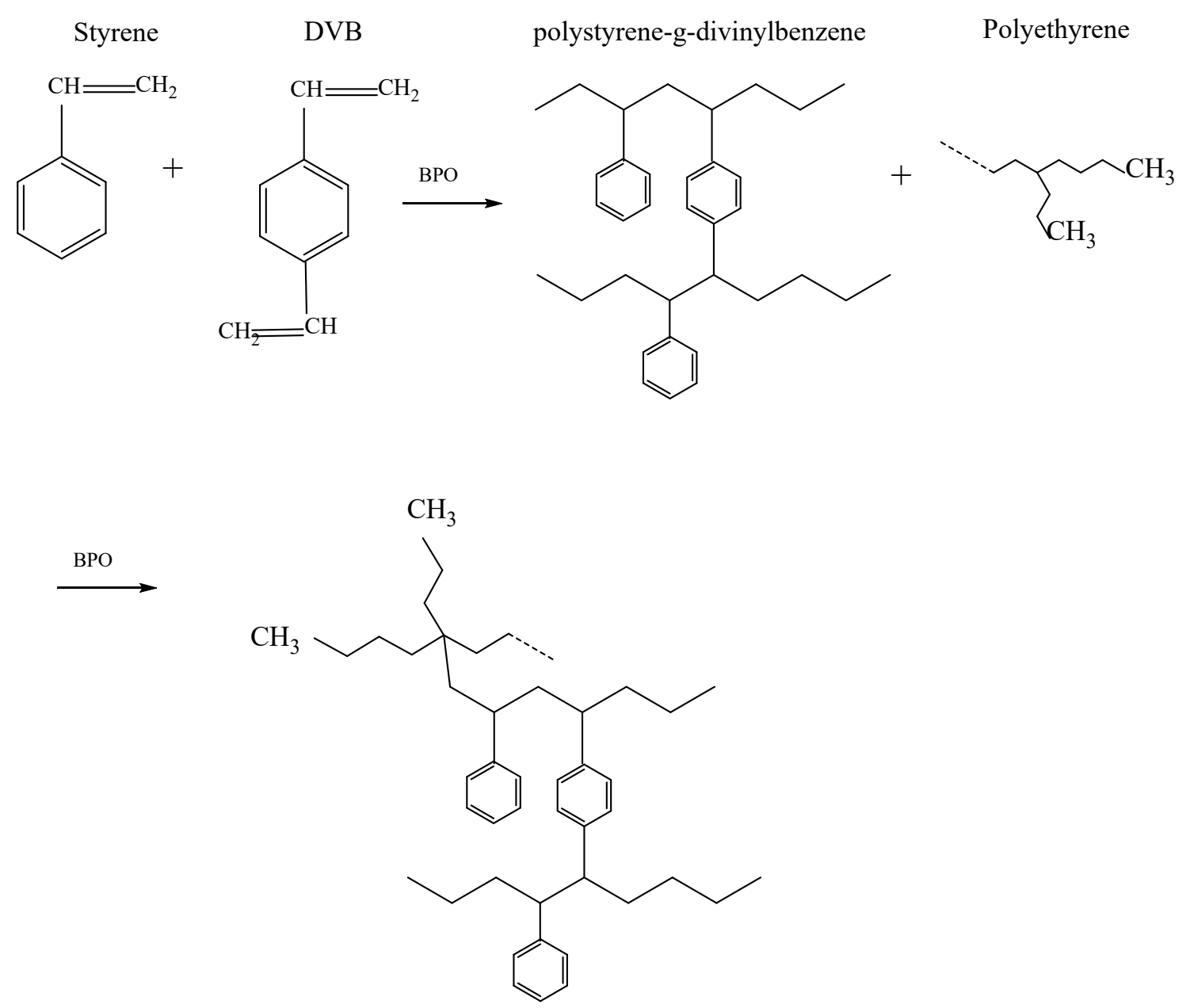

PE-g-PS

Figure 4.13. Polymer compatibilization theory of synthesizing PE-PS copolymer

The copolymer PS-g-DVB fill the interface of PE and can reduce the interfacial tension between PE and styrene. Thus, the PS can be grafted on the PE more easily through compatibilization. But grafted degree is still subject to the amounts of DVB. If increase the addition of styrene, the crosslinked degree must decrease, which result lower PS-gDVB being produced. The graft degree of styrene and IEC will not increase accordingly. The IEC will reach a critical point and then does not change with the styrene increasing. The Figure 4.10 shows the ratio of 1.5 is the critical point. One more thing we need take into consideration is more PS in PE-g-PS film will reduce the flexibility of the material 
since polymers with more benzene rings are mostly rigid. The high addition of styrene is undesirable.

\subsubsection{FT-IR spectrum analysis}

With the amounts of styrene increase (Figure 4.14 from top to bottom), the characteristic peaks of sulfonated PE-g-PS membrane changes accordingly. The two sharp peaks at $1034 \mathrm{~cm}^{-1}$ and $1168 \mathrm{~cm}^{-1}$ represent $\mathrm{SO}_{3}$ groups. The broad bands at $3200-3600 \mathrm{~cm}^{-1}$ are due to the $\mathrm{OH}^{-}$groups of water molecules strongly bound to $\mathrm{SO}_{3}$ groups by hydrogen bonding. The size of these characteristic peaks increase with the increase amounts of styrene until the ratio of styrene to $\mathrm{PE}$ is 1.5 . Moreover, the aromatic $\mathrm{C}-\mathrm{H}$ absorption peaks presented at $1637 \mathrm{~cm}^{-1}$ strengthen with the increase of the styrene addition. However, this strengthening effect for ratio 2.5 and 3 characteristic peaks are not obviously. These results confirm sulfonation permanently modified the chemical structure of the PE-g-PS copolymer by introducing $\mathrm{SO}_{3}$ group on it and the final membranes have a strong hydrophilic nature. One the other hand, with the styrene increasing, the graft degree of PS on PE and functional group $\left(\mathrm{SO}_{3}\right)$ on PS do not always increase accordingly. When the styrene increment reaches the critical point, the increment of graft degree would trend to slow or still. The FTIR result is accord with the IEC titration results.

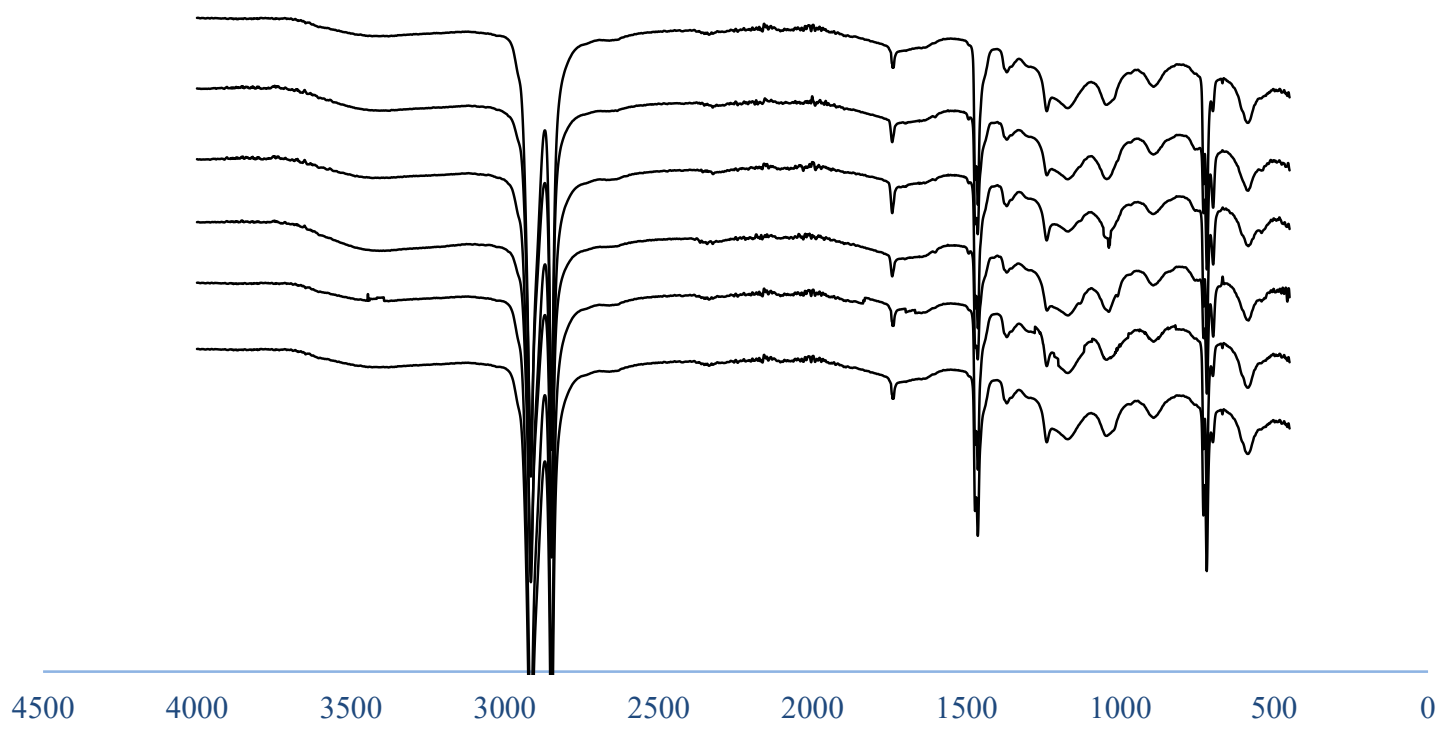

Figure 4.14. FTIR spectra of sulfonation PE-g-PS membrane with different styrene addition (the ratio of styrene to PE from top to bottom is $0.5,1,1.5,2,2.5,3$ ) 


\subsubsection{The effect of the crosslinking degree on cation exchange membrane properties}

According to Kwang-Je Kim's researches, the crosslinking degrees affect the hydrophilicity and mechanical properties of membranes [94]. The hydrophilicity is related on the swelling characteristic and surface properties. Specifically, the membrane swelling degree decreases as the crosslinking degree increase. The membrane surface tension increases with increasing crosslinking degree when it's below 30\%. As the result, the selectivity and flux are also influenced by the crosslinking degree. Studying the effect of crosslink agent on membrane properties can optimize the addition of crosslink agent and can regard this result as the foundation of ions permeation and flux studying. In our research, we kept the ratio of styrene to $\mathrm{PE}$ as 1.5 for all the cation exchange membrane samples. The DVB contents were set to the $0 \%, 3 \%, 6 \%, 9 \%$ of styrene addition to the four various samples. All the samples were added in the same amount of initiator BPO. The mechanical properties (burst strength) and swelling characteristic of membranes were investigated. The FT-IR was also studied to research how crosslinking degree influence membrane properties.

\subsubsection{The effect of the crosslinking degree on IEC}

The effect of crosslinking degree on IEC of cation exchange membrane was investigated. The results presented in Figure 4.15 indicate the IEC value increase firstly and then decrease abruptly as the crosslinking degree increase. The cation exchange membrane with 3\% crosslinking degree obtains the highest IEC. Kwang-Je Kim's research pointed the swelling of membrane for water is great affected by the crosslinking degree [94]. It is expected that the water permeability in ion exchange process decrease with the crosslinking degree increasing. The water permeability largely determines the ions exchange ability due to the ions are dragged through membrane with water permeation. Therefore, the IEC is related on the crosslinking degree. The reason why the IEC value for $3 \%$ crosslinking is greater than that for $0 \%$ is because the compatibilization of PS-gDVB can promote more styrene grafting on PE and enhance IEC value furtherly. 


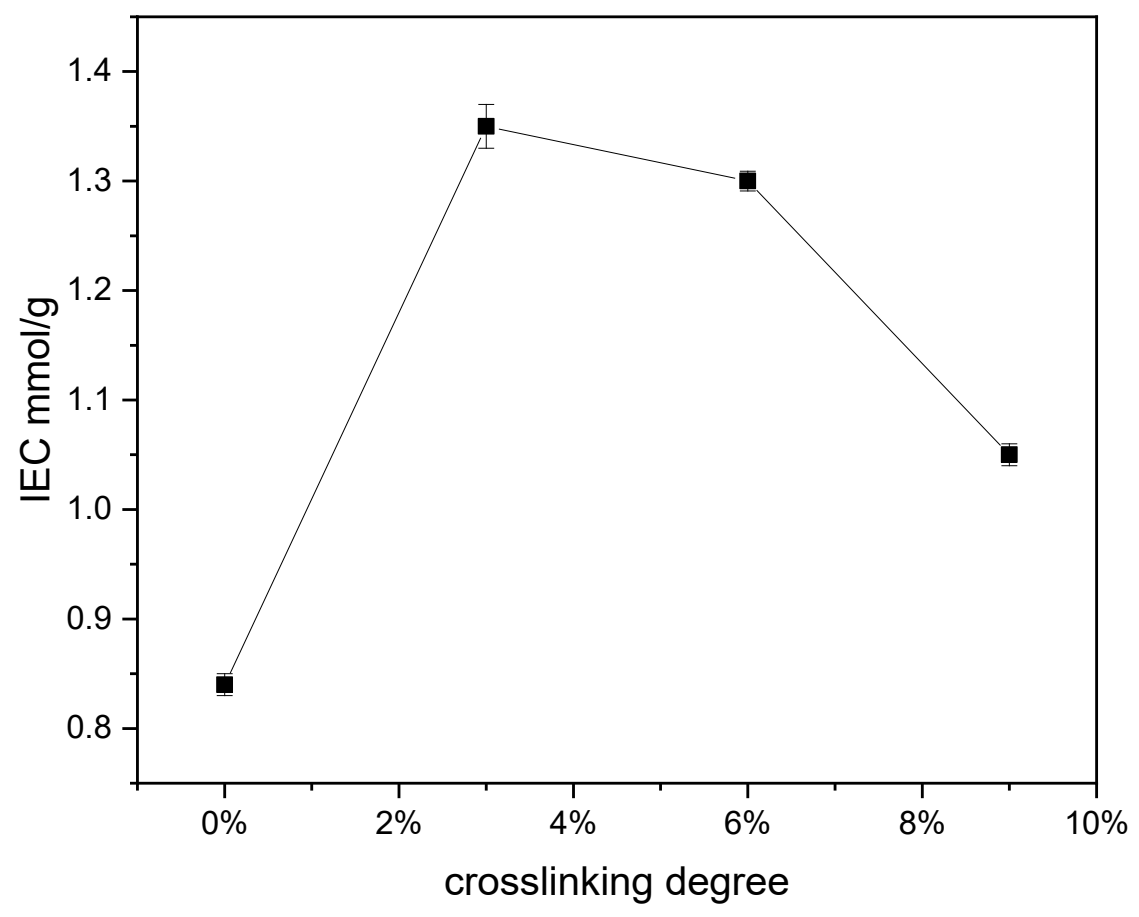

Figure 4.15 IEC of cation exchange membrane with various crosslinking degree

\subsubsection{The effects of the crosslinking degree on burst strength}

The burst strength/thickness values for cation exchange membrane with different crosslinking degree are shown in Figure 4.16. as a function of crosslinking degree. Burst strength is related on the thickness of material. Therefore, the value of burst strength/thickness is the one being worth compared since the uneven thickness for every membrane samples. Figure 4.16 shows the burst strength/thickness increase sharply as the crosslinking degree increase. When the crosslink agent filled into the PE and styrene mixture, it constructs the bonds with PE-g-PS chains. This reaction makes PE-g-PS structure more density and firm. Thus, the membrane with higher degree of crosslinking can resist more impact during the burst strength test. 


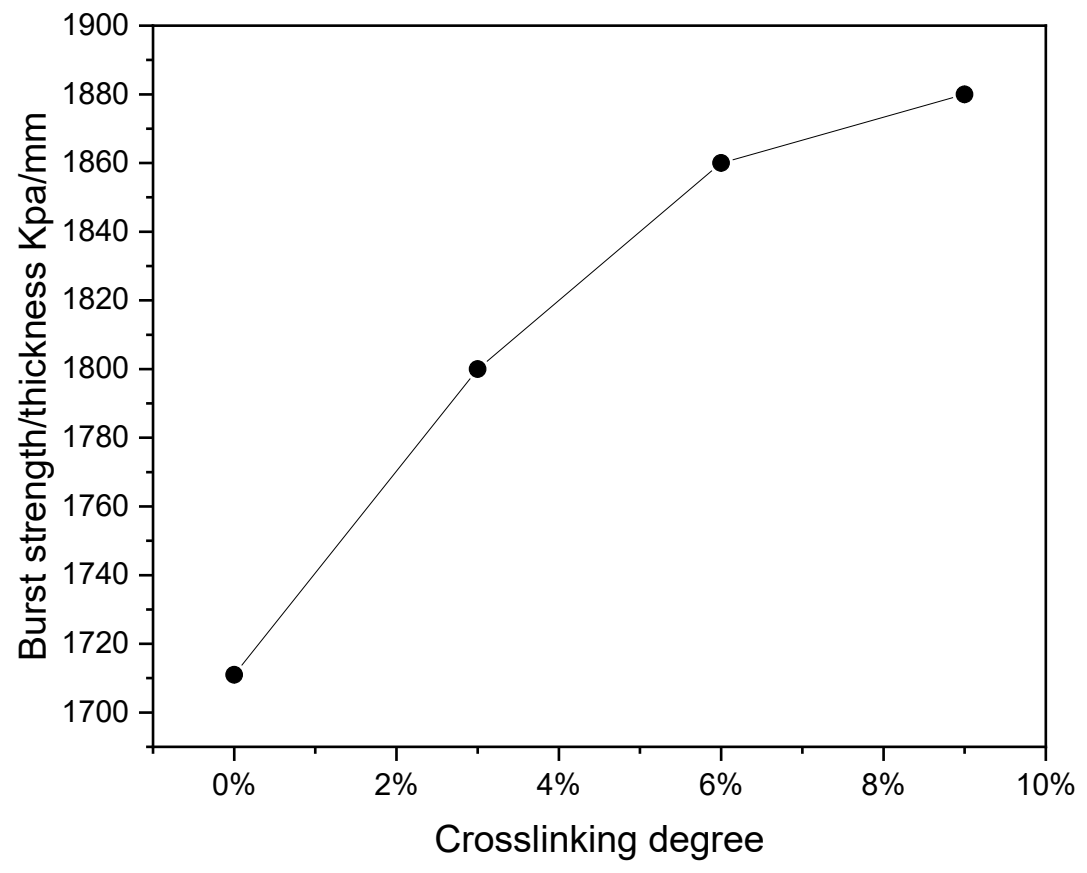

Figure 4.16. Burst strength/thickness of membrane with different crosslinking degree

\subsubsection{FTIR analysis}

The bending vibration bands of the benzene ring planes at $1009 \mathrm{~cm}^{-1}$ (see Figure 4.17.), were believed to be affected by the symmetric stretching vibration of the $\mathrm{S}=\mathrm{O}$ bonds affiliated with the $\mathrm{SO}^{3-}$ groups. Furthermore, the band at $1039 \mathrm{~cm}^{-1}$ is assigned to the antisymmetric stretching vibration of the $\mathrm{S}=\mathrm{O}$ bond. Finally, the $\mathrm{SO}^{3-}$ groups are confirmed to be introduced into the PE-g-PS membrane and the $\mathrm{S}=\mathrm{O}$ stretching vibration bands at $1009 \mathrm{~cm}^{-1}$ decrease as the degree of crosslinking increases. The O-H stretching vibration bans at $3330 \mathrm{~cm}^{-1}$ weakens as the degree of crosslinking increases. This indicates that the appearance of crosslinking agents leads more ionic groups attached on the based membranes, but with increasing the crosslinking agents, leading based membranes attracted less ionic groups. The $3 \%$ crosslinking is optimized ratio. 


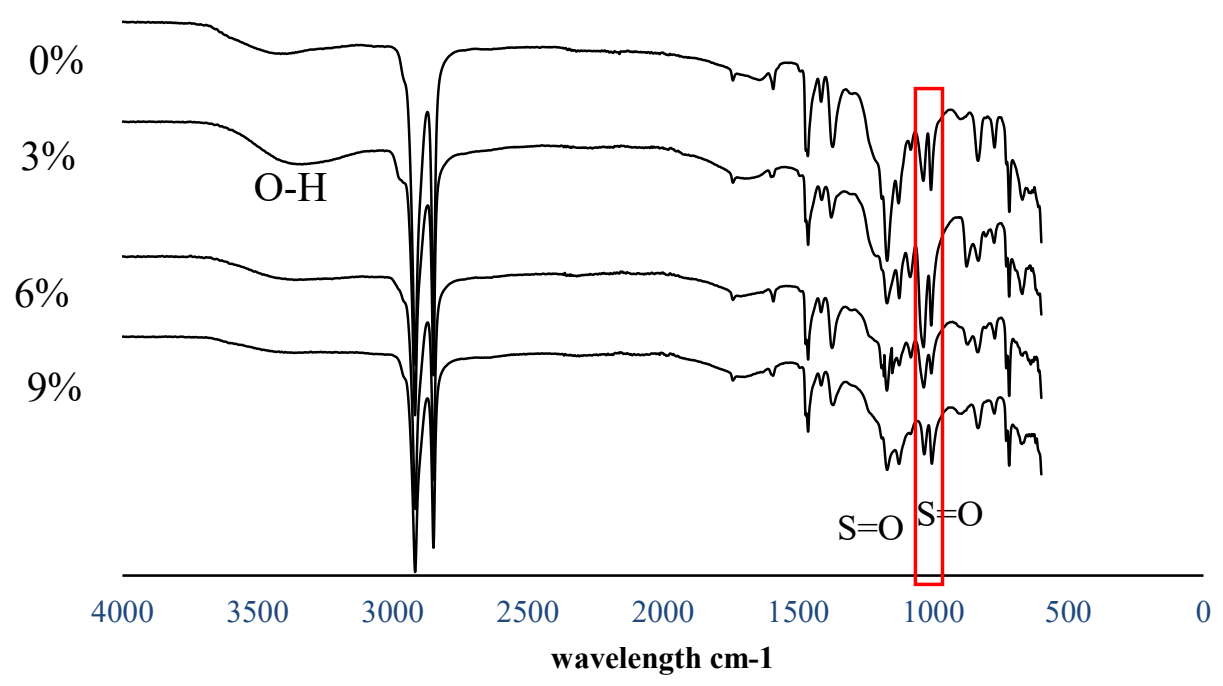

Figure4.17. FTIR spectra of sulfonated PE-g-PS membranes with variational DVB additions

\subsubsection{The effect of solfonaiton condition on cation exchange membrane properties}

Using the classical sulfonation method, the PE-g-PS copolymers were sulfonated to obtain the $\mathrm{SO}_{3}$-groups. The sulfonation duration and the temperature were investigated by IEC test and FTIR analysis to evaluate the effects on sulfonation degree. Three reaction temperatures were investigated in this section, including room temperature, $50^{\circ} \mathrm{C}$ and $70^{\circ} \mathrm{C}$.

IEC test results and FTIR spectrums of sulfonated PE-g-PS membranes with different sulfonation temperatures are presented in Figure 4.18. and 4.19. respectively. Both results show that the higher sulfonation temperature, the more sulfonated anion charged groups were attached on the PE-g-PS membranes.

The bending vibration band appearing at $1028 \mathrm{~cm}^{-1}$ in room temperature is associate the sulfonation spectra. This peak split into two independent peaks $\left(1009\right.$ and $\left.1039 \mathrm{~cm}^{-1}\right)$ for $50^{\circ} \mathrm{C}$ and $70^{\circ} \mathrm{C}$ spectra. This was believed to be affected by the symmetric stretching vibration of the $\mathrm{S}=\mathrm{O}$ bond affiliated with the $\mathrm{SO}_{3}{ }^{-}$group. The fairly broad band at about $3460 \mathrm{~cm}^{-1}$ was assigned to the bending vibration of the $\mathrm{OH}^{-}$group, which was probably caused by the drifting of its stretching vibration toward the low frequency direction, under the hydrogen bond interaction between the $\mathrm{H}_{2} \mathrm{O}$ molecule and the oxygen atom of the $\mathrm{SO}_{3}{ }^{-}$group. The aromatic $\mathrm{C}-\mathrm{H}$ absorption peaks present at $1637 \mathrm{~cm}^{-1}$ strengthen and 
broaden with the increase of the sulfonation degree. Furthermore, the band at $1128 \mathrm{~cm}^{-1}$ presented in $50^{\circ} \mathrm{C}$ and $70^{\circ} \mathrm{C}$ spectra is assigned to the antisymmetric stretching vibration of the $\mathrm{S}=\mathrm{O}$ bond. Thus, the $\mathrm{SO}_{3}{ }^{-}$groups have been confirmed to have been introduced into the PE-g-PS membrane and the sulfonation degree are higher than the room temperature reaction.

As a result, the maximum sulfonation degree existed at $70{ }^{\circ} \mathrm{C}$. Raising the reaction temperature promote the sulfonation rate and degree for cation exchange membrane. However, the IEC result indicates the too high sulfonation temperature, which was above the boiling point $\left(83.5^{\circ} \mathrm{C}\right)$ of catalyst, would weaken the penetration of the concentrated sulfuric into the PE-g-PS membrane. Therefore, we propose that catalyst DCE acted as not only a swelling agent, but also a protectant for the sulfonation process of the PE-g-PS membrane.

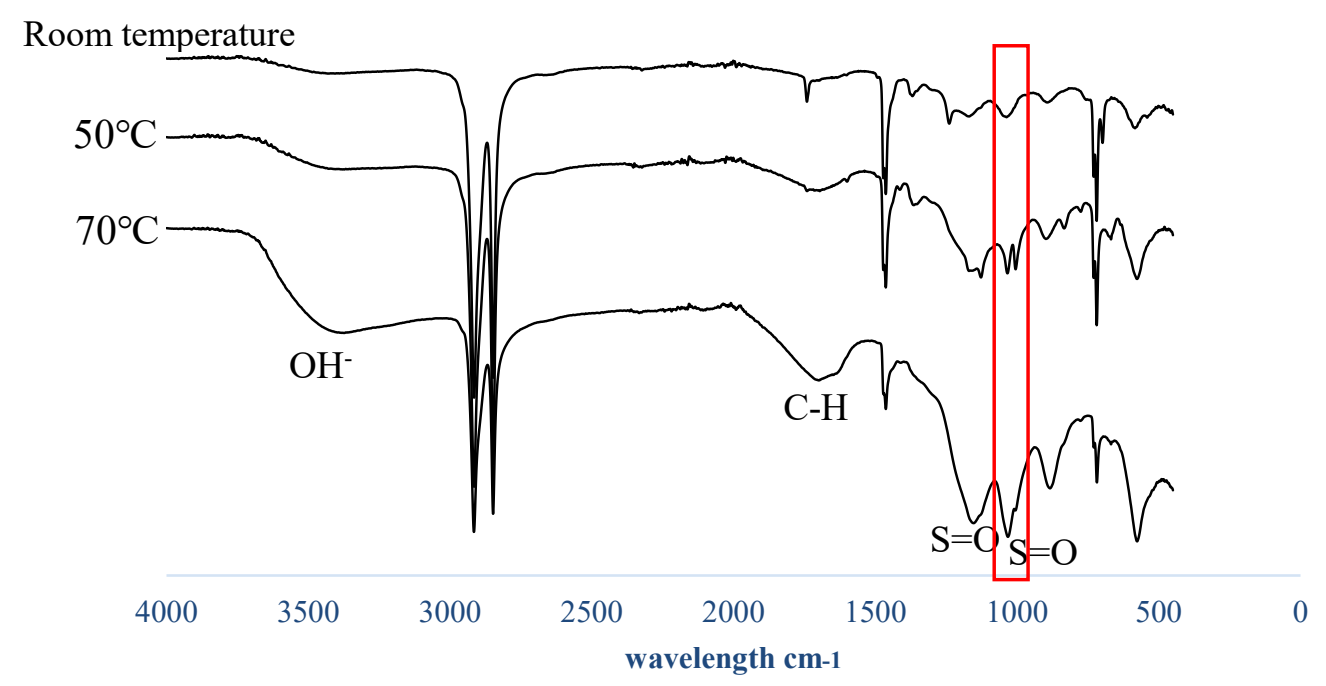

Figure 4.18. FTIR spectra of sulfonated PE-g-PS membranes with different sulfonation temperature (from top to bottom is room temperature, $50^{\circ} \mathrm{C}$ and $70^{\circ} \mathrm{C}$ ) 


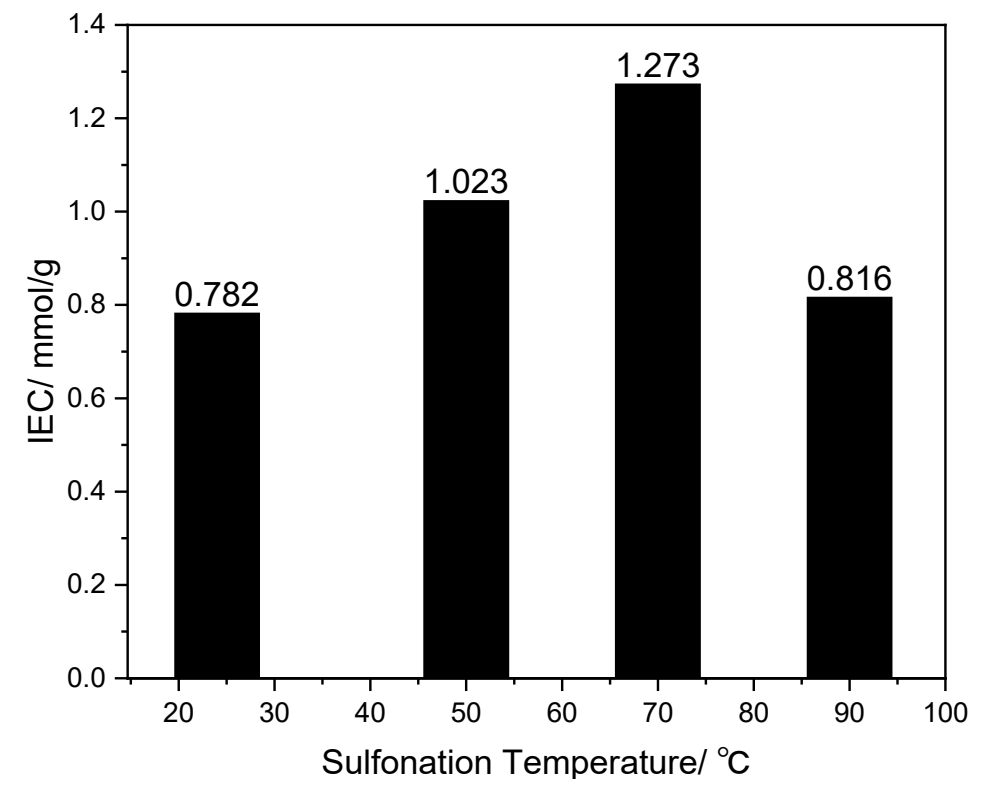

Figure 4.19. IEC value of sulfonated PE-g-PS membranes with different sulfonation temperature

The IEC as a function of sulfonation duration in cation exchange membrane is shown in Figure 4.20. With the elevating sulfonation duration, the IEC value increase at the first 23 hours. However, this increasing trend does not continue and present fluctuation during the 3-5 hours, then the curve is stable after 5 hours. The optimized sulfonation duration is 2-3 hours.

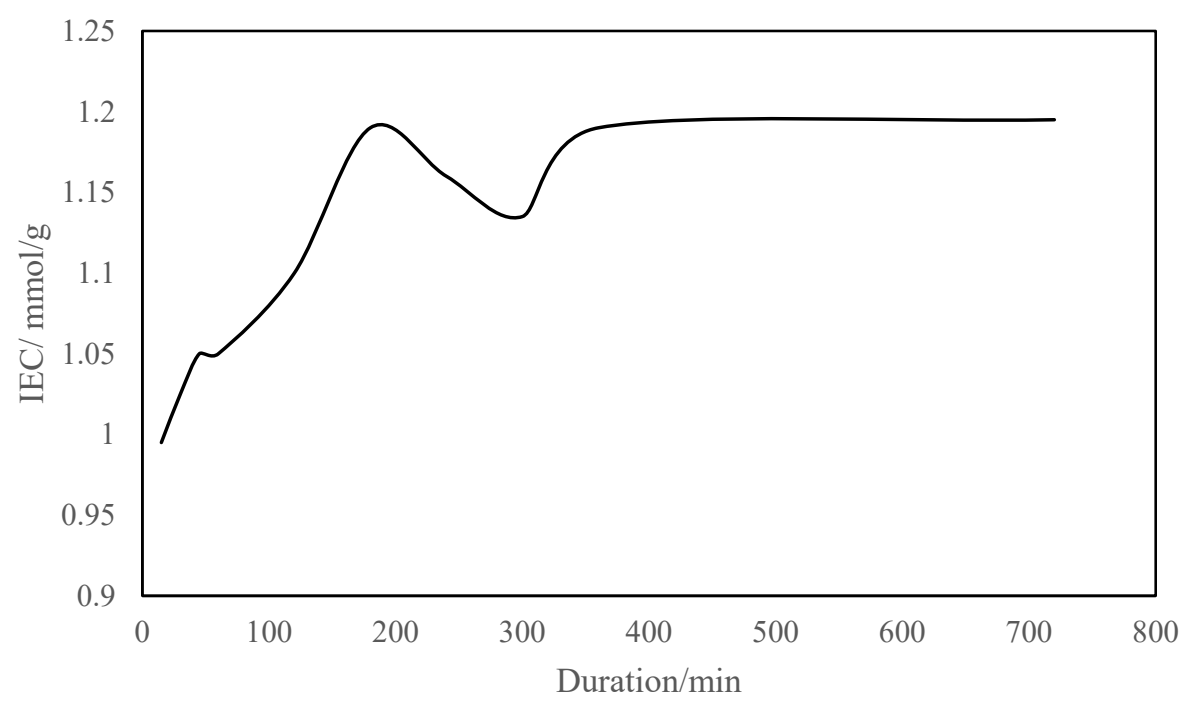


Figure 4.20. IEC of sulfonated PE-g-PS with different sulfonation duration

\subsubsection{The effect of the initiator (BPO) concentration on cation exchange membrane properties}

The BPO initiator has an impact on the grafting degree of polystyrene onto polyethylene. $\mathrm{BPO}$ decomposes to generate two free radicals at $90^{\circ} \mathrm{C}$ (Figure 4.21.). The free radicals can attack either styrene or polyethylene, resulting in polystyrene or polyethylene grafted polystyrene. The grafted copolymer is our aimed synthetic product. Too high BPO concentration will result in a high concentration of free radicals, promoting selfpolymerization of styrene. In this study, different concentrations of BPO $(0.33 \%, 1.00 \%$ and $1.67 \%$ ) were explored to analyze the effect on final properties. Too low of the BPO concentration will not generate enough free radicals, resulting in low reaction rate.

Figure 4.22 indicate that with the BPO concentration increasing, the IEC increase until $1.00 \%$, continually increase BPO concentration results IEC decrease. Therefore, the additive $\mathrm{BPO}$ with $1.00 \%$ concentration is optimized.

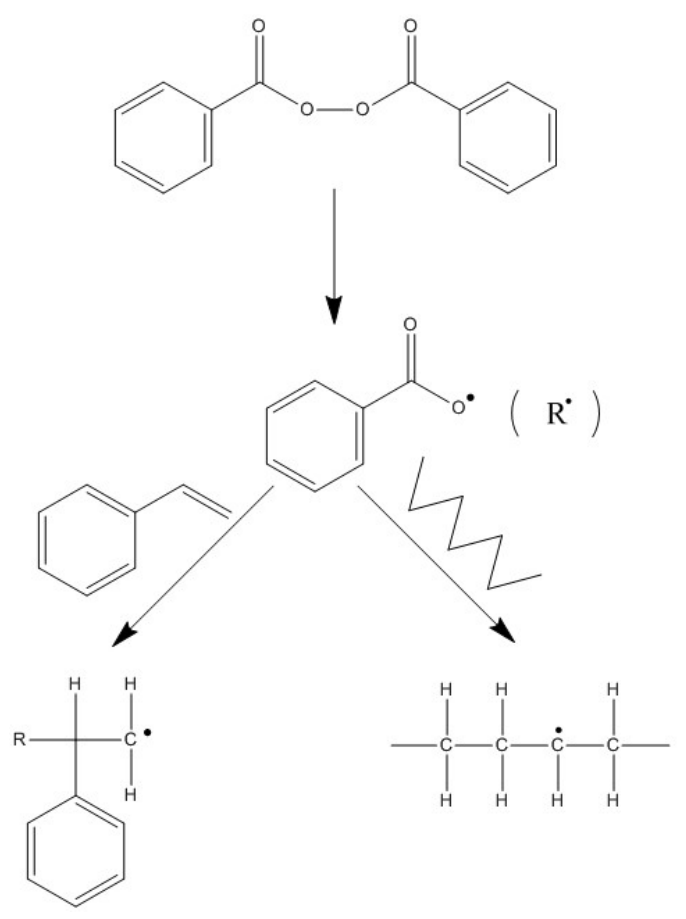

Figure 4.21. BPO decomposing process 


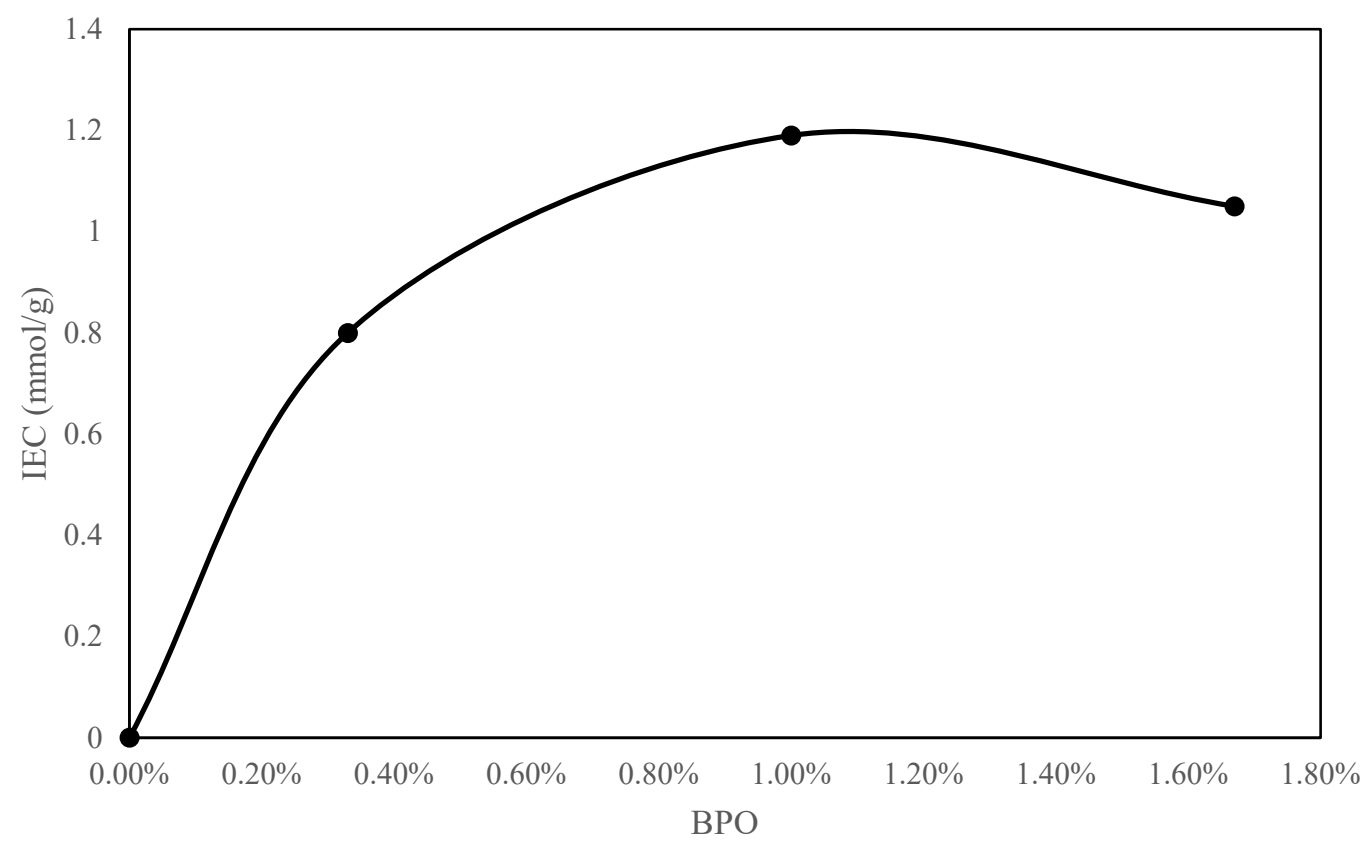

Figure 4.22. IEC values of PE-g-PS cation exchange membranes synthesized from different $\mathrm{BPO}$ concentrations

\subsection{Summary}

The effects of the inert polymer types, the component ratio among monomers, initiator, crosslinked agent, and the reaction conditions on CEM's IEC, water uptake, thermal and mechanical properties and water process efficiency in electrodialysis were investigated in this section. The chemical structure and morphology of synthesized copolymer were characterized by FTIR and SEM, respectively. LDPE was proved to be the optimized inert polymer option compare to LLDPE and HDPE. LDPE possesses the highest graft degree due to its high degrees of branches and low-density structure. PP and PVC based CEM have better mechanical properties (burst strength and tension strength) and thermal expansion restriction than LDPE, but lower IEC value due to low compatibility with PS. The optimized ratio of styrene, LDPE, crosslinking degree and BPO are $1.5,3 \%$ and $1 \%$, respectively in CEM, which obtained the highest IEC value $(1.72 \mathrm{mmol} / \mathrm{g})$ and moderate burst strength. The membrane with a higher degree of crosslinking can resist more impact during the burst strength test. The maximum sulfonation degree existed at $70{ }^{\circ} \mathrm{C}$. Raising the reaction temperature promoted the rate and degree of sulfonation. However, the too high sulfonation temperature (above $83.5^{\circ} \mathrm{C}$ ) would weaken the concentrated sulfuric penetration into the PE-g-PS membrane. 


\section{Investigation the polyolefin-based anion exchange membrane}

\subsection{Introduction}

The IEM were paired up cation exchange membranes (CEM) and anion exchanged membranes (AEM). A typical AEM contains positively charged groups, such as $-\mathrm{NH}_{3}{ }^{+}$, $\mathrm{NRH}^{2+},-\mathrm{NR}_{2} \mathrm{H}^{+},-\mathrm{NR}_{3}{ }^{+},-\mathrm{PR}_{3}{ }^{+},-\mathrm{SR}_{2}{ }^{+}$, etc., fixed to the membrane backbone and allows the passage of anions but reject cations $[95,96]$. As an ideal application for advanced separated membranes technology, AEMs are desired to maintain high ion exchange capacity and mechanical properties under various conditions, such as different temperature and $\mathrm{pH}$ environment. Compared with cation exchange membranes, typical AEMs have lower ion exchange capacity due to the lower hydrophilic nature of $\mathrm{OH}^{-}$and weaker dissociation of ionic functionality.

Just like CEM, the desired properties of AEM were determined by the inert backbone polymers, reactive polymers and methods. Polyolefin films such as polyethylene has been proven to be an attractive inert polymer material to produce IEM since they have properties of high mechanical, good chemical and thermal stabilities. There are two methods to graft different reactive polymers (monomers) on inert polymer. In a traditional way, styrene was used as the monomer to copolymerize on PE molecules. Preparing PE-g-PS base membrane for functionalization by different groups is the basic idea to cation and anion membranes. Our CEM was made in this way by being treated in concentrated sulfuric acid. We also used this traditional method to make AEM by treating PE-g-PS base membranes in chloromethyl ethyl ether $\left(\mathrm{ClCH}_{2}-\mathrm{O}-\mathrm{CH}_{2} \mathrm{CH}_{3}\right)$ and

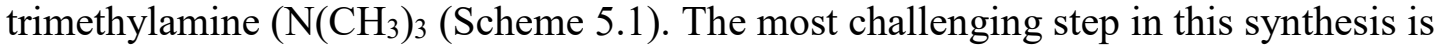
chloromethylation. Common chloromethylation reagents such as chloromethyl ethyl ether or chloromethyl methyl ether is known to be carcinogenic. $10 \mathrm{~mL}$ chloromethyl ethyl ether was used to make one piece of anion membranes which renders a huge risk. From the safety perspective, this method should be quitted [97].

In some recent studies, researchers use 4-vinylbenzyl chloride as the monomer for the synthesis AEM [98]. 4-Vinylbenzyl Chloride (VBC), which contains chloromethyl groups in its structure, was reported to be an alternative reactive polymer to replace chloromethyl methyl ether (CMME) or chloromethyl ethyl ether (CMEE) reagents to avoids a harmful chloromethylation reaction [99,100]. In this research, 4-vinylbenzyl chloride is another alternative monomer to be studied grafted on the low-density polyethylene (LDPE) inert polymer via non-radiation polymerization followed by quaternization and alkalization; benzoyl peroxide as an initiator and divinylbenzene as cross linkage agent (scheme 5.2.). There are several advantages of this method. Firstly, we can avoid using very toxic chloromethyl ether which makes the new synthetic process "greener". At the same time, the four-step process is simplified to a three-step process. 
What's more, by using pure VBC as the starting material, we can guarantee quaternarization and thus enough functional groups on the membrane.

However, the AEM properties of using 4-vinylbenzyl chloride as monomer to synthesis did not reach our expectation. The styrene was introduced as co-monomers (Scheme 5.3.). It was proved that it can be copolymerized with divinylbenzene to be regarded as compatibilizer in spite of the introduction of styrene could not bring any contribution to functional groups grafted, The copolymer of polystyrene and divinylbenzene can improve the adhesion and miscibility between PE and VBC since there are low miscibility between them. The membranes were characterized in terms morphological analysis, FTIR analysis, elemental analysis and thermal stability analysis. The ion exchange capacity, mechanical properties, water uptake and thermal stabilities were tested.

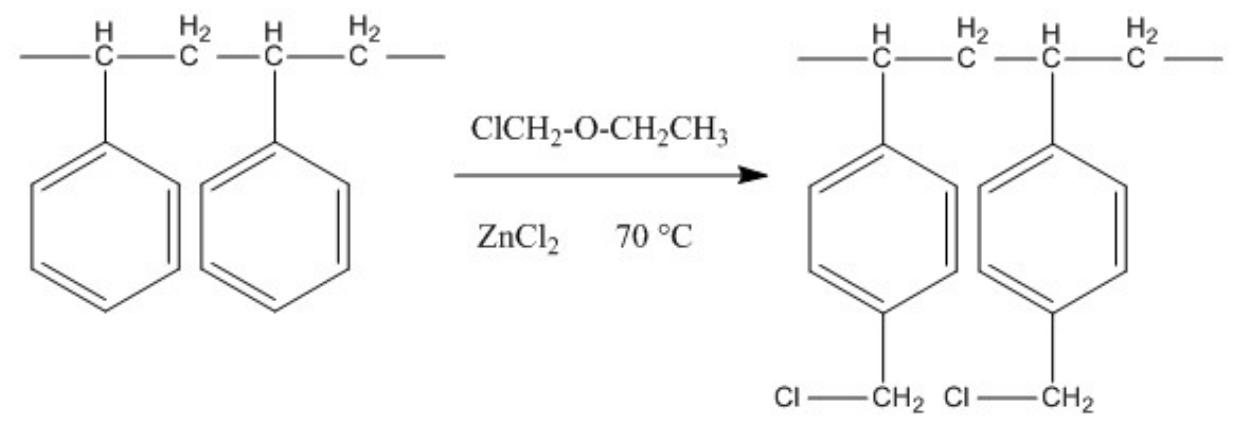

Chloromethylation

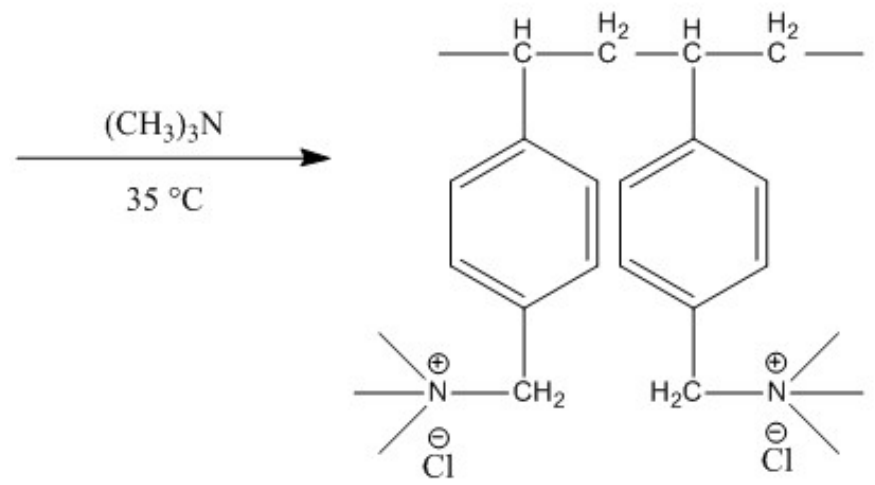

Quaternarization

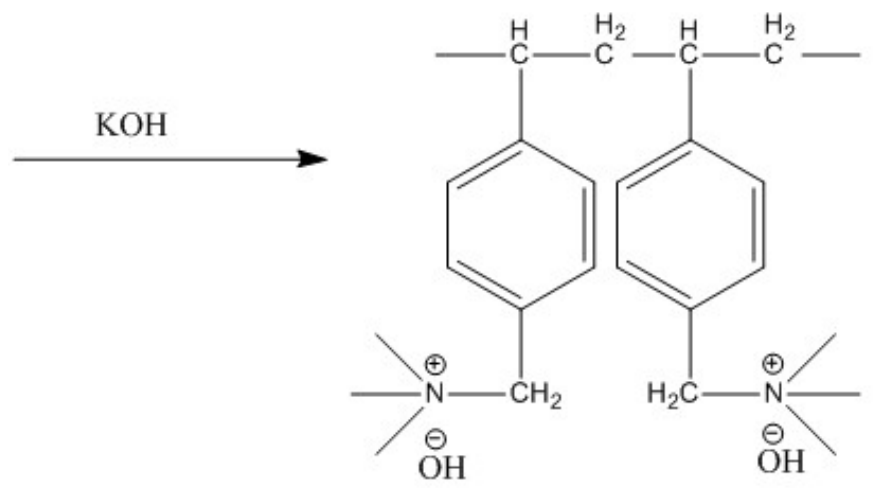

Alkalization 
Scheme 5.1. Traditional synthetic route of AEM

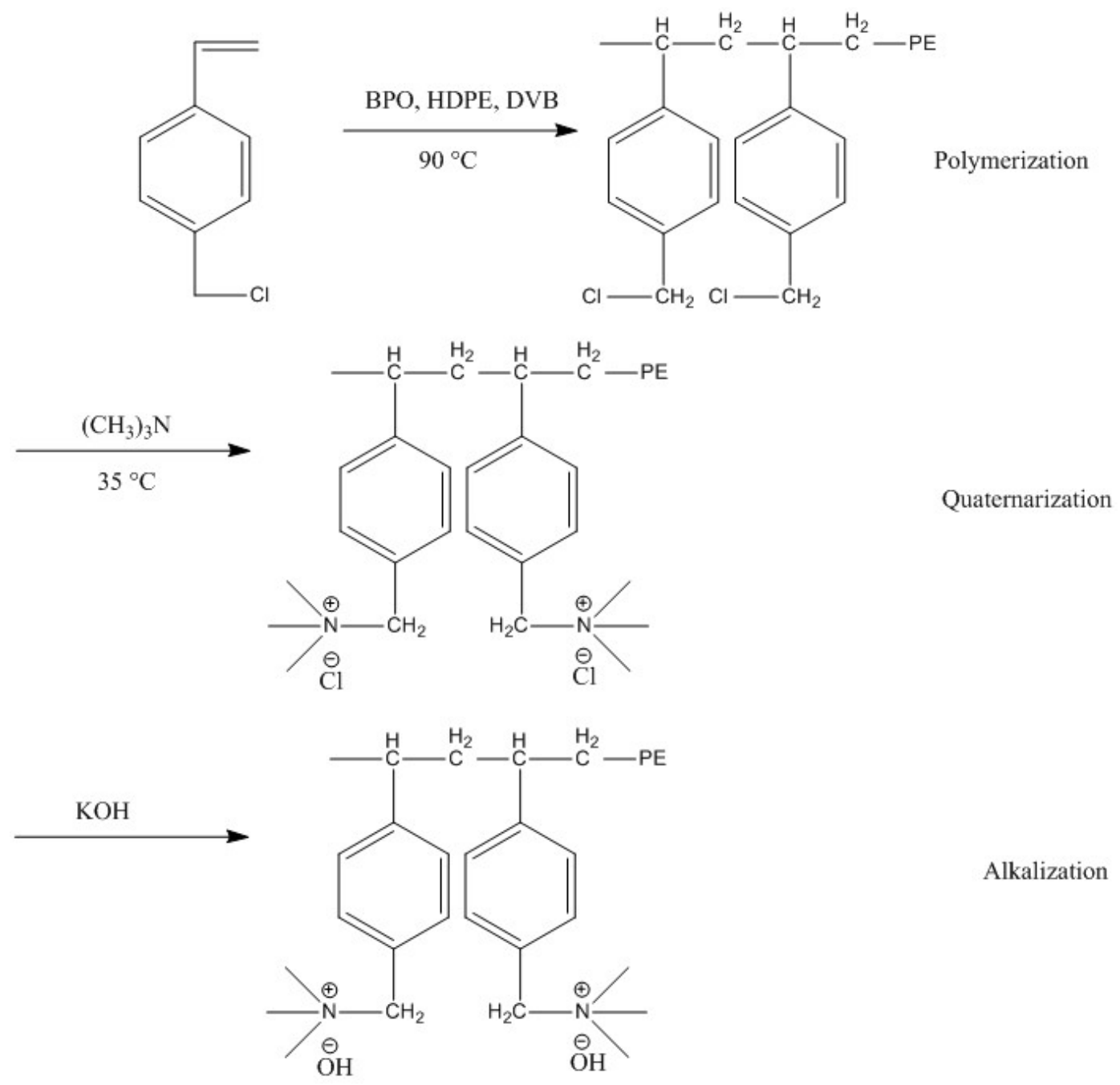

Scheme 5.2. AEM synthetic route of using VBC as monomer 

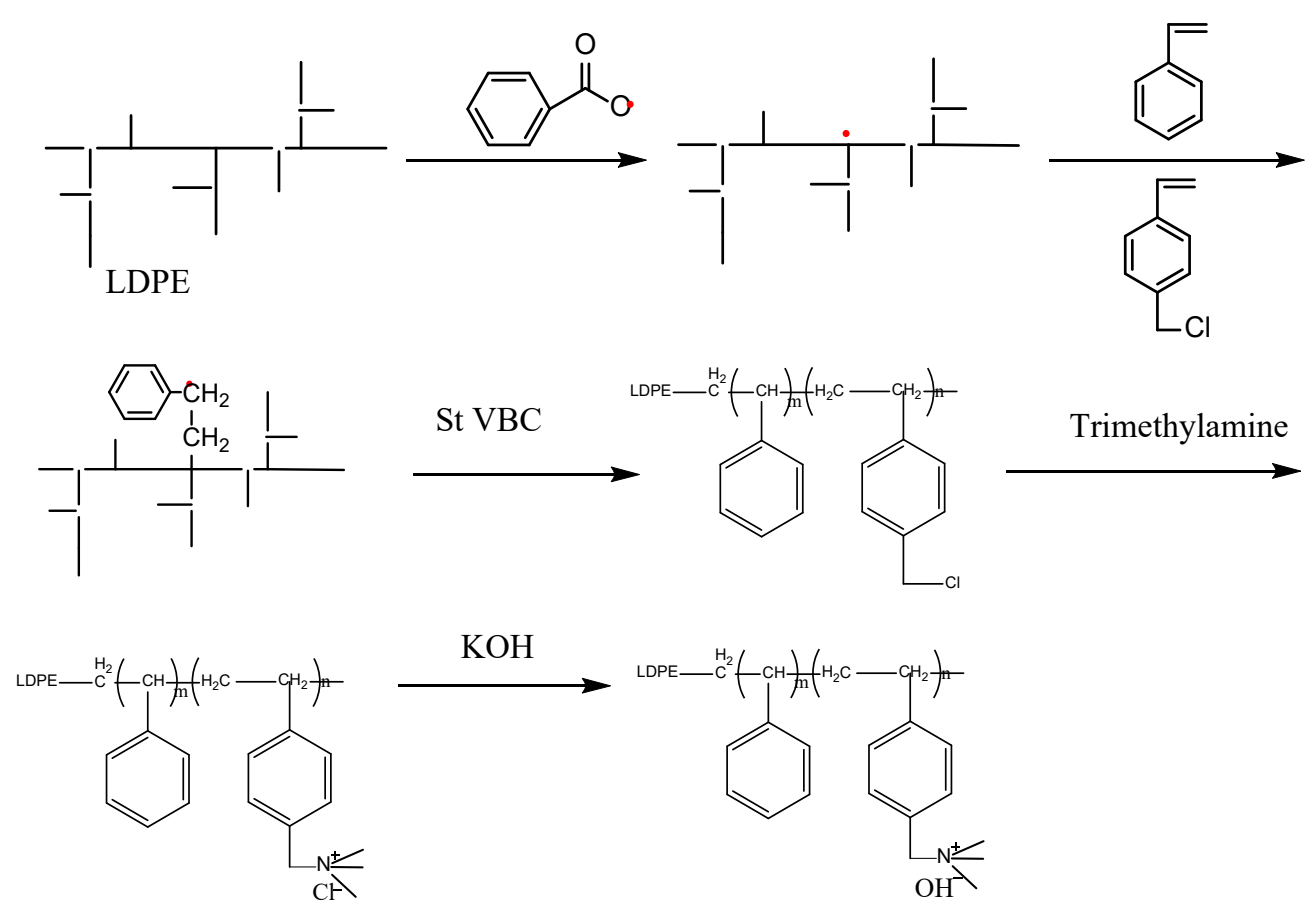

Scheme 5.3. AEM synthetic route of using VBC and styrene as co-monomer

\subsection{Comparation of synthesizing methods}

\subsubsection{Chloromethylation method to synthesize AEM (PE-g-PS based AEM)}

Step 1: PE-g-PS co-polymer film was prepared as described in 3.2.1.1.

Step 2: Chloromethylation

$0.5 \mathrm{~g}$ of PE-g-PS film was cut into small pieces and place in a flask. $15 \mathrm{ml} \mathrm{1,2-}$

dichloroethane was added and heated to $70{ }^{\circ} \mathrm{C}$ to swell the polymer for $1 \mathrm{~h} .0 .05 \mathrm{~g} \mathrm{ZnCl} 2$ and $0.5 \mathrm{ml}$ chloromethyl ethyl ether was added and the reaction was stirred at $70{ }^{\circ} \mathrm{C}$ for two hours. A pale-yellow material was obtained (Figure 5.1.), washed with methanol and water. 


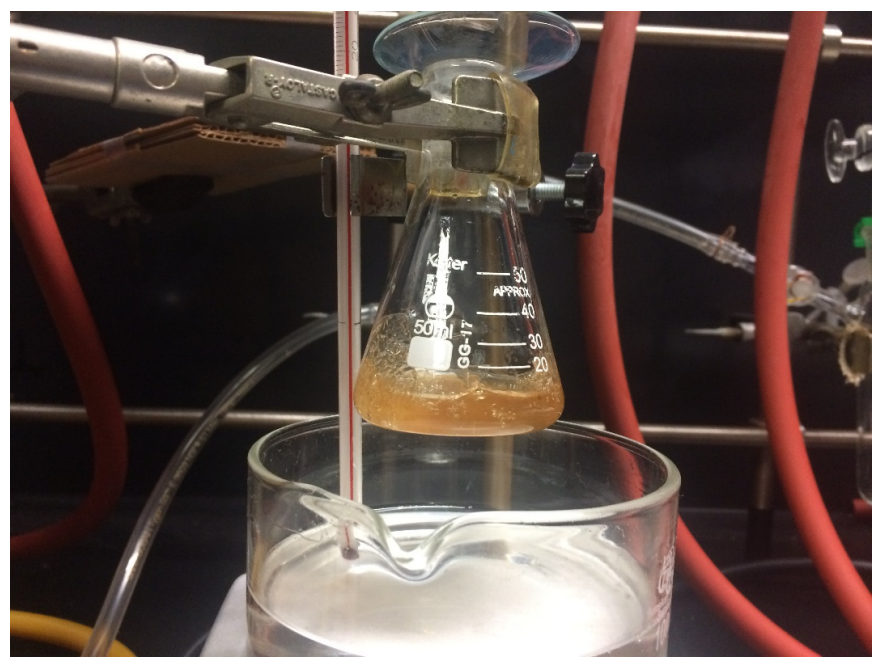

Figure 5.1. PE-g-PS film turns yellow in chloromethylation

\section{Step 3: Quaternarization}

The film was then stirred in $20 \mathrm{ml}$ trimethylamine solution at $35^{\circ} \mathrm{C}$ overnight. Then the film was then washed with distilled water multiple times.

Step 4: Alkalization

The film was then soaked in $1 \mathrm{M} \mathrm{KOH}$ solution at room temperature overnight to substitute the chloride with the hydroxide group. The film was then washed with water and dried under air at ambient temperature. A white or yellow film was obtained depending on the period for chloromethylation.

\subsubsection{4-Vinylbenzyl Chloride method to synthesize AEM (PE-g-PVBC based AEM)}

Step 1: $2 \mathrm{~g}$ of PE was placed in a two-neck flask with $5 \mathrm{ml}$ xylene and $20 \mathrm{ml}$ toluene. The mixture was evacuated and flushed with nitrogen three times to insure the inert atmosphere for the reaction. The mixture was then heated to $95{ }^{\circ} \mathrm{C}$ to dissolve PE film.

Step 2: When the solution turned clear, $2.2 \mathrm{~g} \mathrm{VBC}$ and $0.1 \mathrm{ml} \mathrm{DVB} \mathrm{(3 \% )} \mathrm{were} \mathrm{added} \mathrm{to}$ the flask using an air-tight syringe. The mixture was stirred at $95{ }^{\circ} \mathrm{C}$ for $2 \mathrm{~h}$ and the initiator $0.01 \mathrm{~g}$ BPO $(0.5 \%)$ was added. The reaction was stirred at $95{ }^{\circ} \mathrm{C}$ for another $4-5$ $\mathrm{h}$. The solution was then concentrated and cast onto glass slides, resulting in semitransparent films.

Step 3: Quaternarization 
Step 4: Alkalization

\subsubsection{4-Vinylbenzyl Chloride and styrene co-monomer method to synthesize AEM (PE-g-PS/PVBC based AEM)}

AEM includes 4-Vinylbenzyl Chloride and styrene co-monomer as co-monomer was prepared as described in 3.2.2.

\subsection{Experimental results and discussion}

\subsubsection{The effect of chloromethylation reaction condition on anion exchange membrane properties}

Researchers used chloromethyl methyl ether and chloromethyl ethyl ether to functionalize the PE-g-PS membranes. This reaction is usually catalyzed by Zinc chloride which serves as a Lewis acid in this reaction. However, no detail about this reaction can be found in any literature according to best of my knowledge. To understand the chloromethylation reaction, two reactions were set up: one in chloromethane and the other in chloromethane and ethanol mixture (Table 5.1.). Zinc chloride is soluble in ethanol, but insoluble in chloromethane. The two experiments were stirred at $55^{\circ} \mathrm{C}$ for 6 hours. The solid was taken out from the mixture, washed with methanol and distilled water and dried at $60^{\circ} \mathrm{C}$. The resulting samples were treated with trimethylamine at $35^{\circ} \mathrm{C}$ overnight, washed and dried. The samples show different color as we can see from Figure 5.2. The samples from heterogeneous catalysis is pale yellow, while the homogeneous catalysis is snow white (Figure 5.3.). The two samples were analyzed by CHNS elemental analyzer. The results show that the catalytic reaction in chloromethane has a $\mathrm{N}$ content of $1.39 \%$ which is higher than the reaction under chloromethane and ethanol mixture $(0.84 \%)$. This means the IEC of AEM catalyzed from chloromethane is higher than the one synthesized from chloromethane and ethanol mixture. Therefore, chloromethane was used to prepare our anion exchange membranes.

Table 5.1. $\mathrm{ZnCl}_{2}$ catalytic chloromethylation in different solvents

\begin{tabular}{lll} 
solvents & chloromethane & chloromethane + ethanol \\
\hline solubility of catalyst $\mathrm{ZnCl}_{2}$ & insoluble & soluble \\
\hline catalysis type & heterogeneous & homogeneous \\
\hline color & pale yellow & snow white \\
\hline $\mathrm{N}$ content & $1.39 \%$ & $0.84 \%$ \\
\hline
\end{tabular}




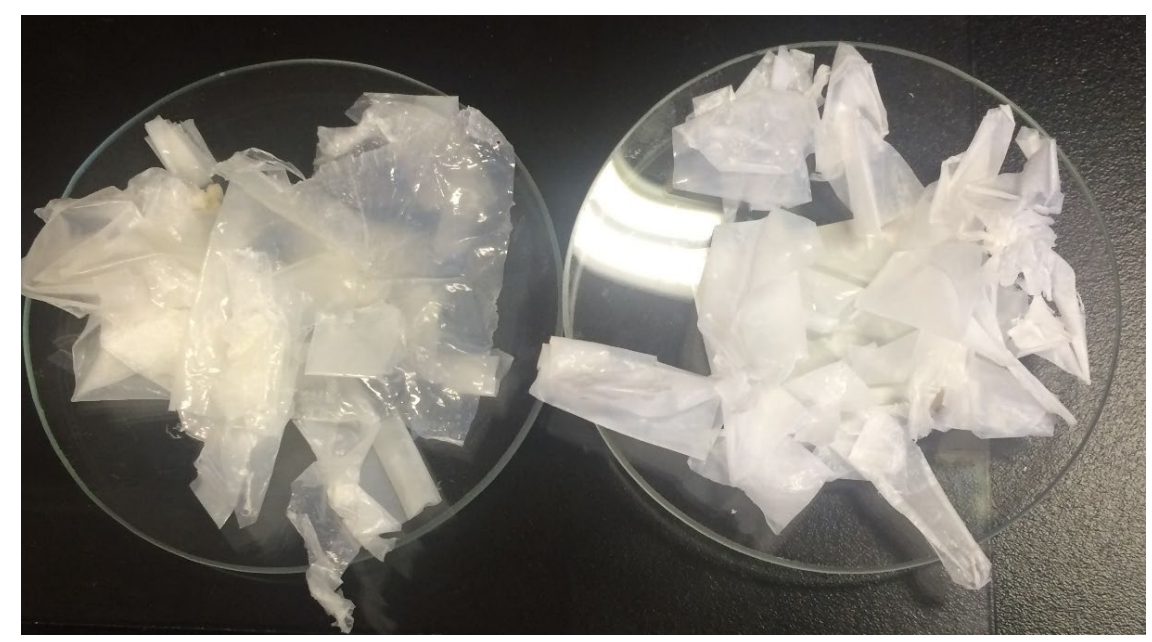

Figure 5.2. Comparison of chloromethylation reaction in chloromethane (left) and chloromethane-ethanol mixture (right)

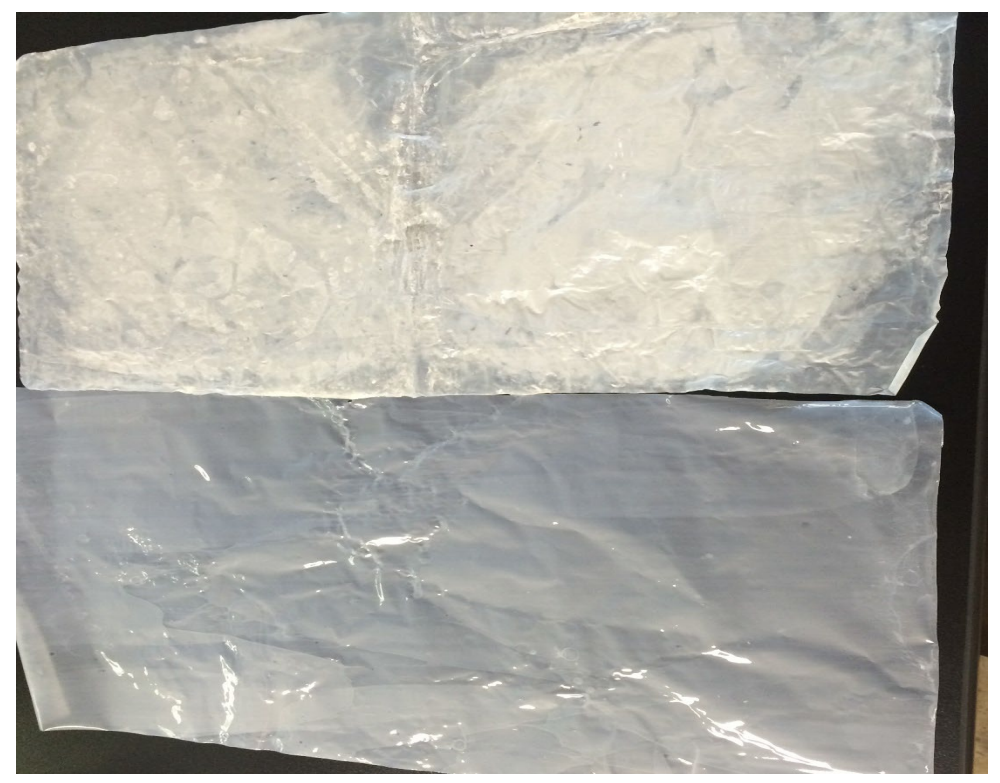

Figure 5.3. Anion exchange membrane synthesized by method 1 (top) and PE-g-PS membrane (bottom) 
We also obtained the infrared spectrum of those AEM (Figure 5.4.). We are mostly interested in quaternary ammonium cation groups. According to literature, these peaks are not strong and likely to show up in $800-900 \mathrm{~cm}^{-1}$ range. In our anion exchange membranes, some new peaks at 890,859 and $824 \mathrm{~cm}^{-1}$ appeared which indicate the quaternary ammonium functional groups. The spectrum of anion exchange membrane 2 which was chloromethylated at $50^{\circ} \mathrm{C}$ for $24 \mathrm{~h}$ show stronger peaks than anion exchange membrane 1 which was chloromethylated at $70^{\circ} \mathrm{C}$ for $2 \mathrm{~h}$.

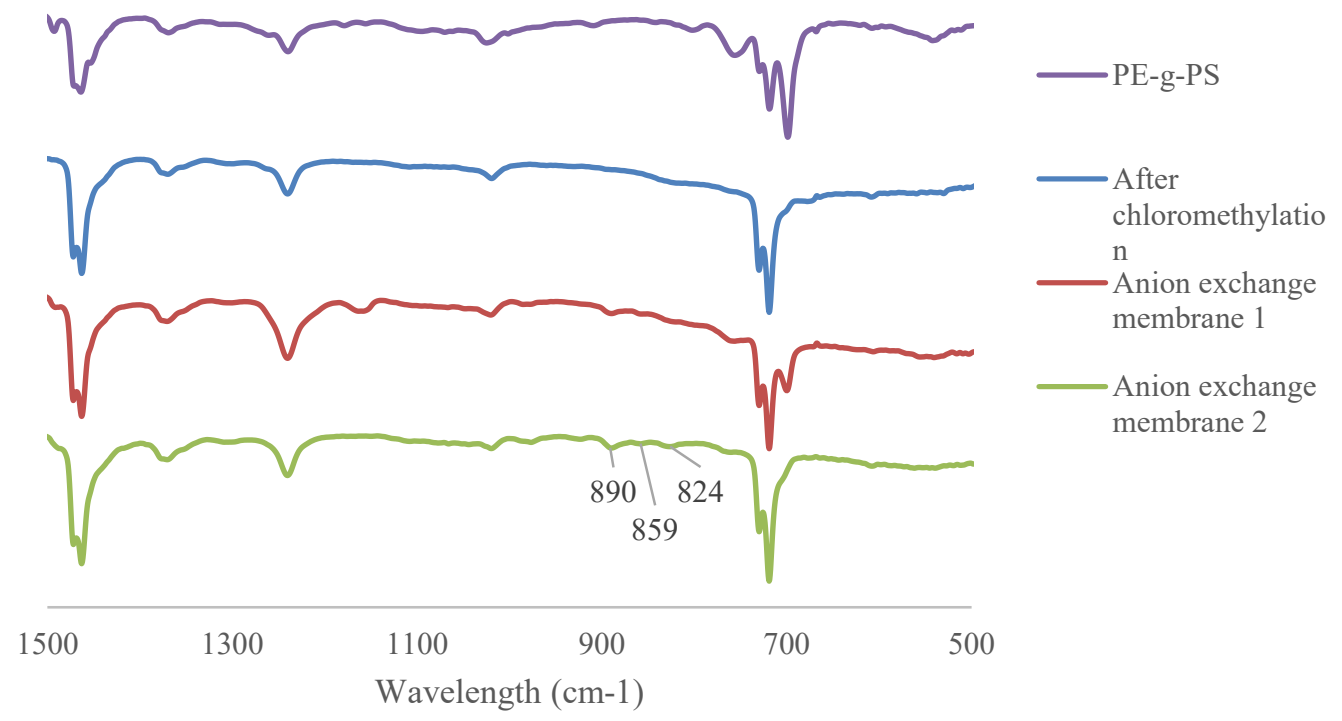

Figure 5.4. Infrared spectra of anion exchange membranes

\subsubsection{PE-g-PVBC based AEM}

4-Vinylbenzyl chloride method was used to prepare a batch of anion membranes. The infrared spectrum shows that the idea to use $\mathrm{VBC}$ as the starting monomer is successful (Figure 5.5.). The pattern is quite like the anion exchange membrane previously synthesized from styrene. The signature region for quaternary ammonium groups (891, 859 and $828 \mathrm{~cm}^{-1}$ ) is much stronger than the previous one, indicating more functional groups on the membrane.

The elemental analysis of these membranes shows a nitrogen content of $0.94 \%$ which corresponds to an IEC value of $0.67 \mathrm{mmol} / \mathrm{g}$. The actual ion exchange capacity was determined to be $0.165 \mathrm{mmol} / \mathrm{g}$ by exchanging with $0.25 \mathrm{M} \mathrm{Na}_{2} \mathrm{SO}_{4}$ overnight. The IEC value is $0.13 \mathrm{mmol} / \mathrm{g}$ if exchanged with $0.5 \mathrm{M} \mathrm{NaCl}$. It is puzzling that $\mathrm{AEM}$ do not show the effect as expected. An explanation would be insufficient functional groups attached 
on the membranes. Based on this speculation, those membranes were put back into trimethylamine solution and treated at higher temperature $\left(70^{\circ} \mathrm{C}\right)$ to investigate if the trimethylation condition influence the functional groups attaching. The FTIR shows evidence of trimethylamine (Figure 5.6.). Peaks at 3379,891 and $853 \mathrm{~cm}^{-1}$ correspond to quaternary amine groups. However, there is not much difference between the anion membrane aminated once or twice in the FTIR spectrum. Moreover, compare with the PE-g-PS based AEM, little improvement was observed on the PE-g-PVBC based AEM when test the working performance in electrodialysis (Figure 5.7.). Those results indicated animation is not the reason for insufficient functional groups.

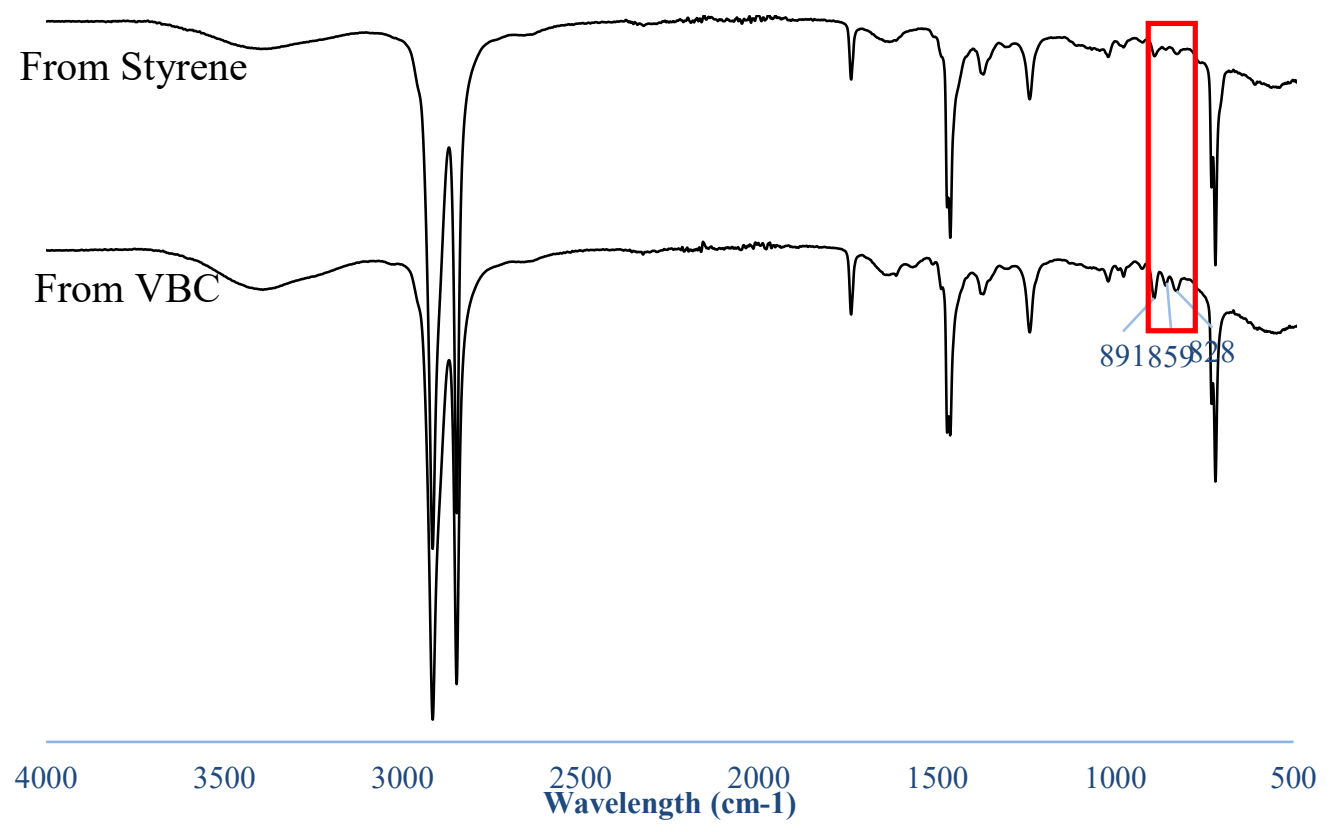

Figure 5.5. FTIR patterns of PE-g-PS based anion exchange membrane synthesized from styrene and $\mathrm{VBC}$ 


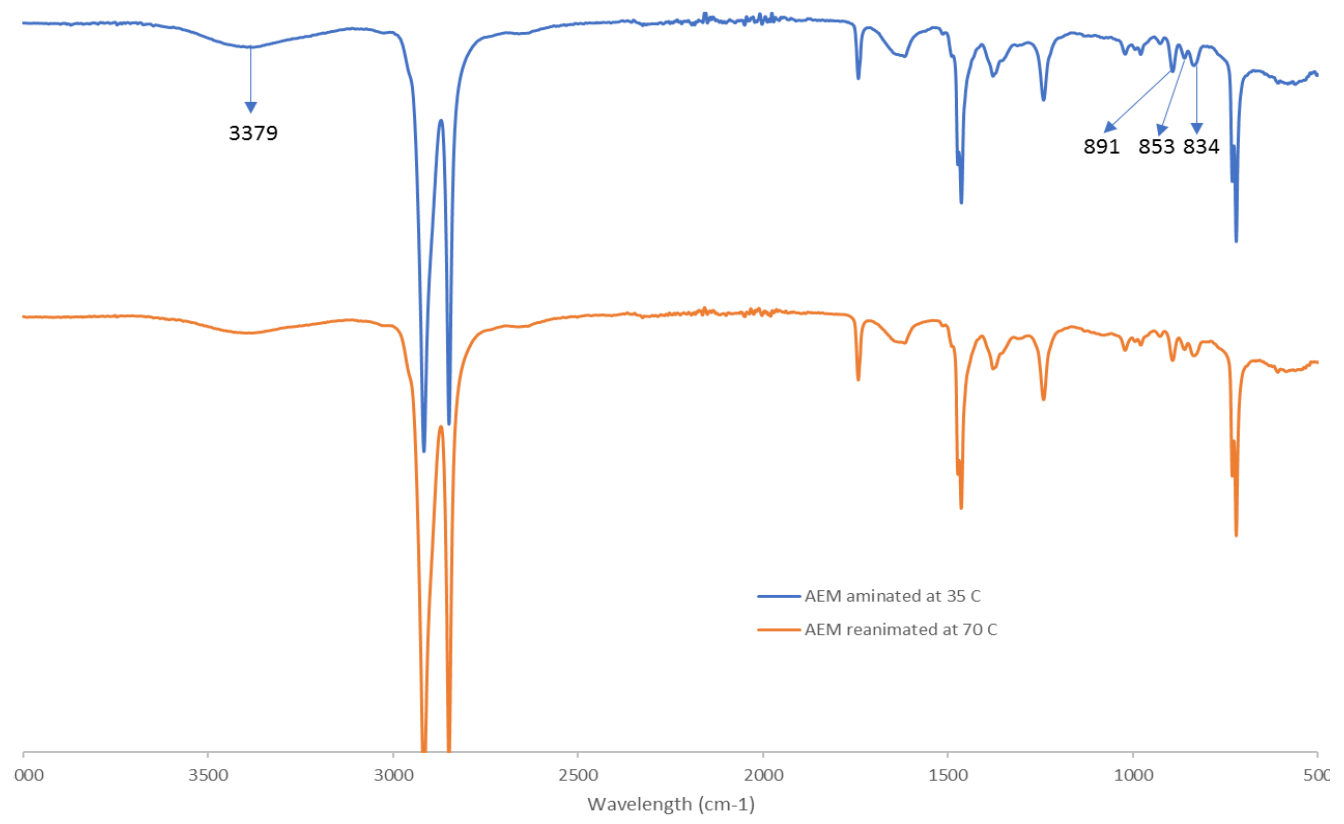

Figure 5.6. FTIR spectrum of PE-g-PVBC based AEM with different amination temperature

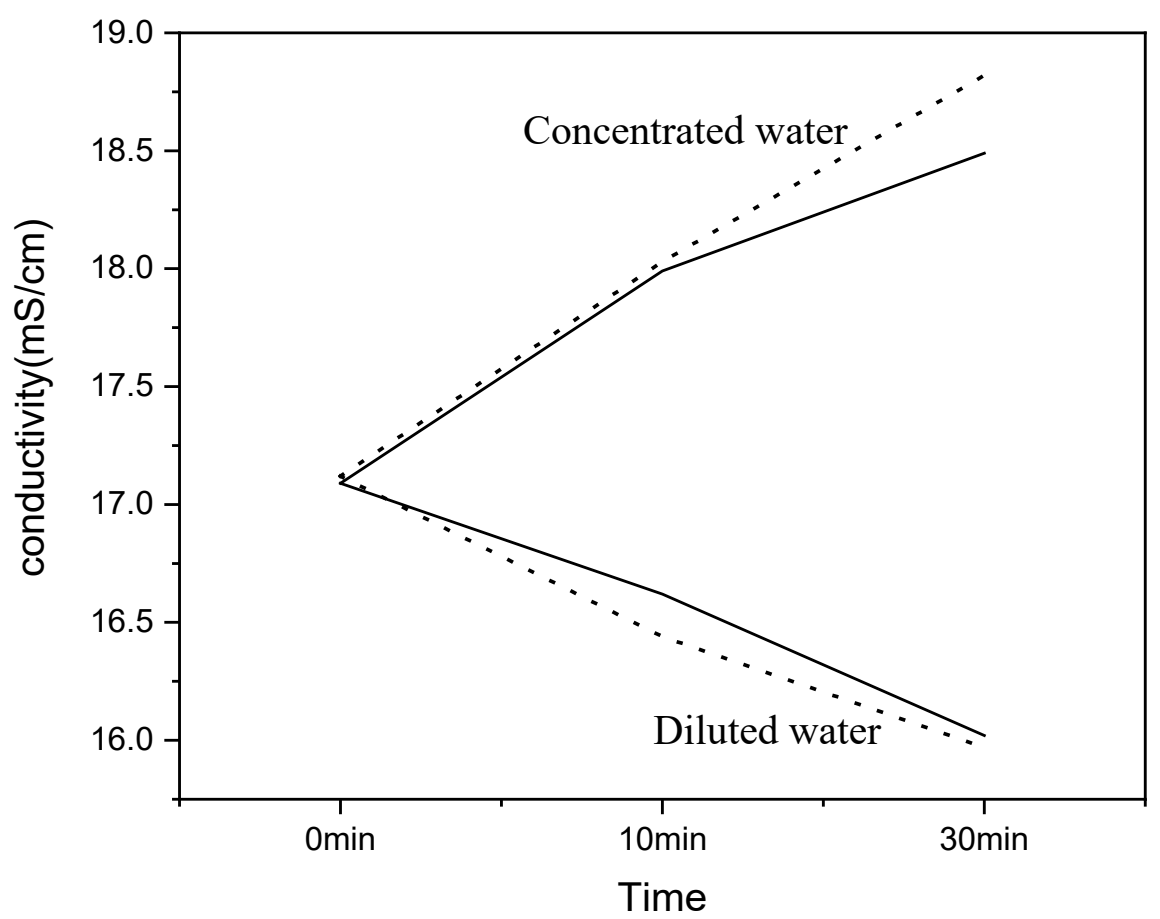


Figure 5.7. Comparation of AEM synthesized from two method for electrolyte conductivity tested in electrodialysis (solid line is PS-g-PS based AEM, dot line is PE-gPVBC based AEM)

Rapid evaporation of $\mathrm{NH}_{3}$ might be another reason that resulted in insufficient amination. To solve this problem, pieces of PE-g-PVBC membranes were soaked in $\mathrm{NH}_{3} \cdot \mathrm{H}_{2} \mathrm{O}$ in sealed containers with tight plastic cover. The reactors were then placed in the oven and heated at $40{ }^{\circ} \mathrm{C}$ for $24 \mathrm{~h}$ and $72 \mathrm{~h}$. After amination reaction, the membranes were washed with distilled water and dried in oven for analysis.

The FTIR analysis is very evident to show the completion of amination after $24 \mathrm{~h}$ reaction at $40{ }^{\circ} \mathrm{C}$ (Figure 5.8.). A literature search shows that the peaks at $1266 \mathrm{~cm}^{-1}$ and $675 \mathrm{~cm}^{-1}$ are associated with wagging and stretching of $\mathrm{C}-\mathrm{Cl}$ bond. Both peaks disappear after $24 \mathrm{~h}$ amination at $40^{\circ} \mathrm{C}$, revealing that the $\mathrm{C}-\mathrm{Cl}$ bonds were completely replaced by amine groups. This is also supported by new peaks at 890,859 and $829 \mathrm{~cm}^{-1}$. There is no difference between the samples treated with $24 \mathrm{~h}$ and $72 \mathrm{~h}$ in FTIR. In conclusion, amination is complete after $24 \mathrm{~h}$ reaction at $40{ }^{\circ} \mathrm{C}$ and this step should not be the reason for insufficient functional groups.

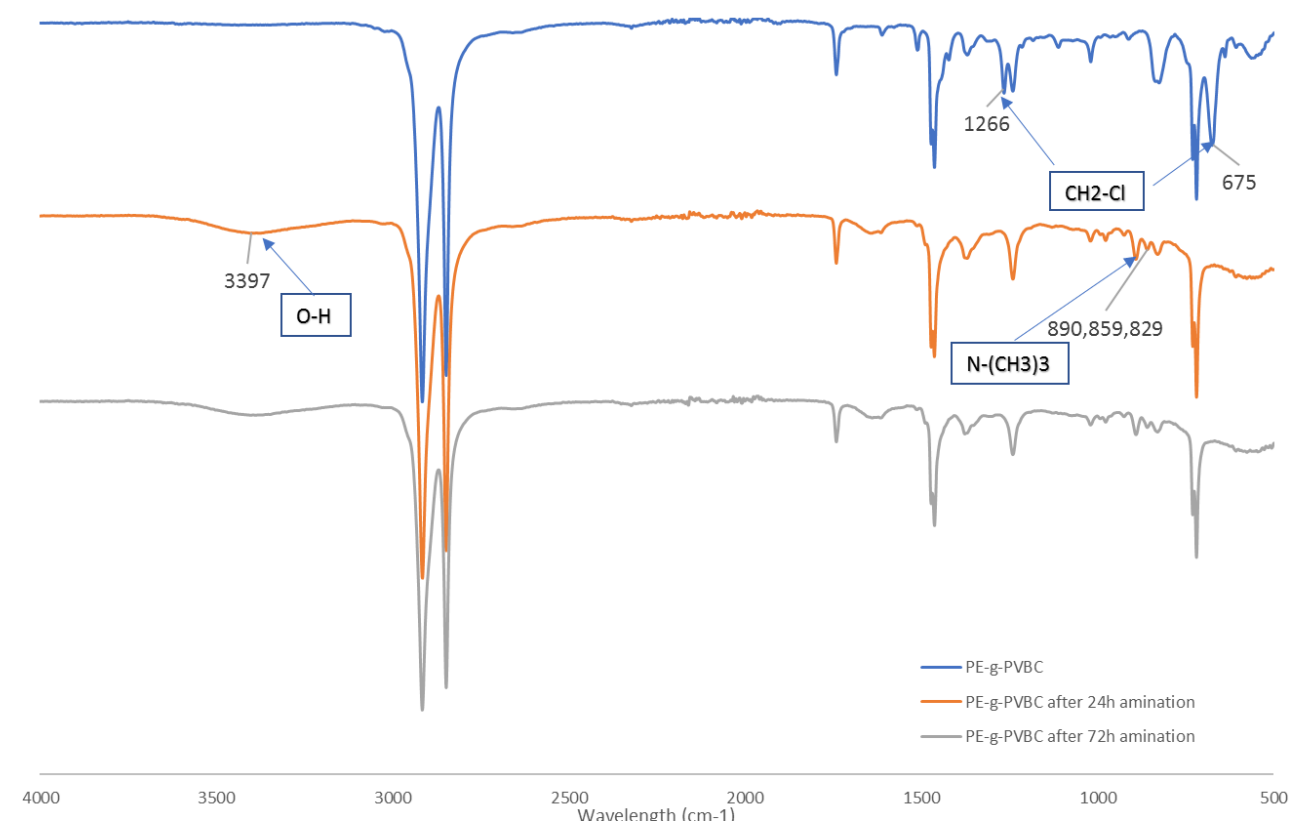

Figure 5.8. FTIR of PE-g-PVBC based membrane, membranes after $24 \mathrm{~h}$ and $72 \mathrm{~h}$ amination at $40{ }^{\circ} \mathrm{C}$

An explanation would be only a small portion of functional trimethylamine groups is working as expected. In this case, the grafting polymerization of 4-vinylbenzyl chloride onto PE membrane causes the insufficient functional groups. One thing to note is 
doubling the amount of VBC monomer increased the actual IEC value from 0.028 $\mathrm{mmol} / \mathrm{g}$ to $0.165 \mathrm{mmol} / \mathrm{g}$. This is still not enough to make good anion exchange membranes. It seems that VBC has low reactivity towards PE membranes. In conclusion, the VBC monomer amount is a factor that results in insufficient functional groups on AEM and should be optimized. To solve this problem, we can try to use a mixture of styrene and VBC as monomers, hoping that styrene can help connect VBC and PE film.

\subsubsection{The effect of styrene on non-radiation grafting PE-g-PVBC AEM}

To study the effect of styrene on graft degree of VBC on PE film, the styrene to VBC mass ratios were varied for given PE films. The various mass ratios included $0,1: 9,2: 8$, 3:7, 4:6 were chosen to be studied. Table 5.2. shows the PE-St-PVBC copolymerization condition with a different monomer ratio.

Table 5.2. A preliminary study of copolymerization condition

\begin{tabular}{ccccc} 
Composition & LDPE & Styrene: VBC & DVB & BPO \\
\hline 1 & $2.5 \mathrm{~g}$ & $0: 2 \mathrm{~g}$ & $0.15 \mathrm{~g}$ & $0.02 \mathrm{~g}$ \\
\hline 2 & $2.5 \mathrm{~g}$ & $1: 9$ & $0.15 \mathrm{~g}$ & $0.02 \mathrm{~g}$ \\
\hline 3 & $2.5 \mathrm{~g}$ & $2: 8$ & $0.15 \mathrm{~g}$ & $0.02 \mathrm{~g}$ \\
\hline 4 & $2.5 \mathrm{~g}$ & $3: 7$ & $0.15 \mathrm{~g}$ & $0.02 \mathrm{~g}$ \\
\hline 5 & $2.5 \mathrm{~g}$ & $4: 6$ & $0.15 \mathrm{~g}$ & $0.02 \mathrm{~g}$ \\
\hline
\end{tabular}

\subsubsection{Characterization of AEM by FTIR spectroscopy analysis}

FTIR spectrum analysis is used to illustrate the characteristic structures of synthesized PE-St-VBC copolymer and functionalized AEM. The chemical structure of the original polyethylene, co-polymer PE-St-PVBC with various styrene addition were analyzed and represented in Figure 5.9. The original PE spectrum is characterized by the methylene strength and bends. Four sharp peaks can reveal the presence of methylene, the $2917 \mathrm{~cm}^{-1}$ and $2848 \mathrm{~cm}^{-1}$ peaks reveal the asymmetry stretching and symmetry stretching of $-\mathrm{CH}_{2}$ respectively. The $-\mathrm{CH}_{2}$ deformation stretching and bending are shown at $1464 \mathrm{~cm}^{-1}$ and $719 \mathrm{~cm}^{-1}$. These four peaks are also presented in the spectrums after copolymerization, indicated the reservation of methylene groups. After copolymerization, some new absorption bends appear at $1608 \mathrm{~cm}^{-1}$ and $1515 \mathrm{~cm}^{-1}$, corresponding the stretching of $\mathrm{C}=\mathrm{C}$ aromatic double bonds. The new peaks which appear at $678 \mathrm{~cm}^{-1}$ are assigned to $\mathrm{C}-\mathrm{Cl}$ stretching and the peak at $1267 \mathrm{~cm}^{-1}$ is due to $-\mathrm{CH}_{2} \mathrm{Cl}$ wagging, both resulting from the $\mathrm{CH}_{2} \mathrm{Cl}$ group present in $\mathrm{VBC}$. These can clearly indicate that the $\mathrm{VBC}$ monomers are 
successfully grafted on the PE films. Note that the peak at the $842 \mathrm{~cm}^{-1}$, corresponding the $\mathrm{C}=\mathrm{C}-\mathrm{H}$ comes from either styrene or $\mathrm{VBC}$ was observed in all copolymer spectrums, and the peaks at 10St and 20St addition show a little sharper than others.

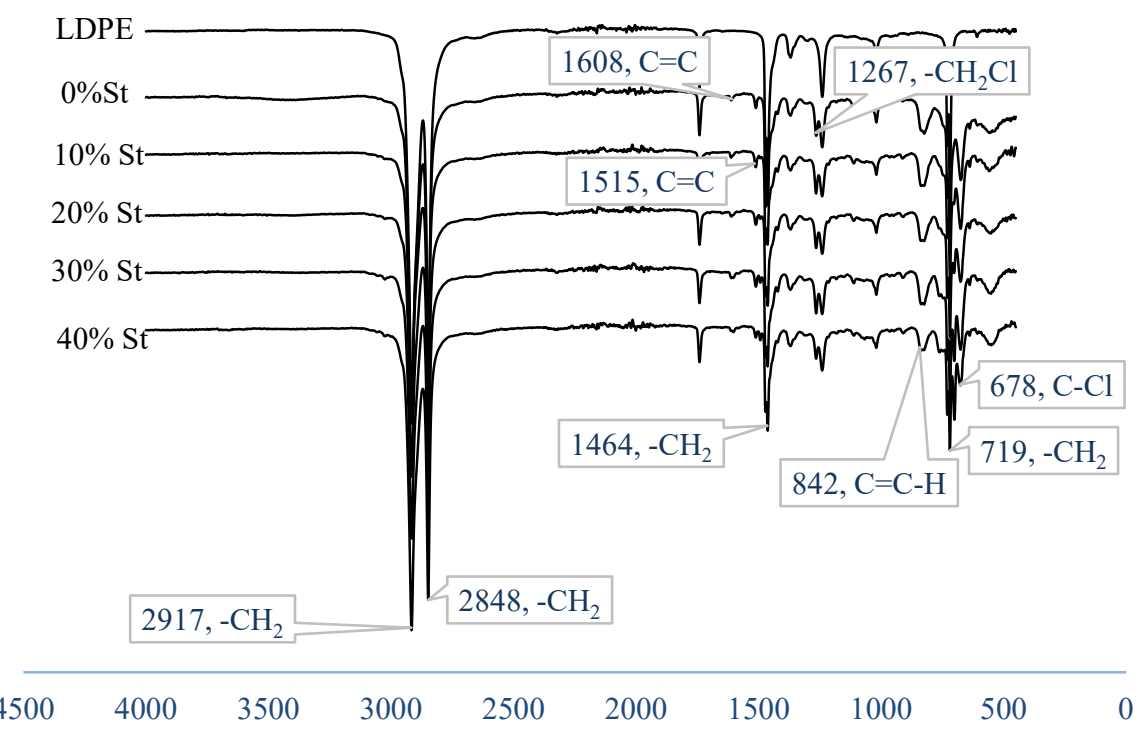

Figure 5.9. FTIR spectra of the original LDPE film (top) and graft copolymers synthesized with different $\mathrm{St}$ : VBC ratios (second from top to bottom St: $\mathrm{VBC}=0: 100$, 10:90, 20:80, 30:70, 40:60).

After quaternarization, the FTIR spectrum at Figure 5.10. shows the $\mathrm{C}=\mathrm{C}$ stretching vibration is shifted to $1632 \mathrm{~cm}^{-1}$, and some characterized peaks of copolymer PE-St$\mathrm{PVBC}$, such as $\mathrm{C}-\mathrm{Cl}$ stretching at $678 \mathrm{~cm}^{-1}$ and $-\mathrm{CH}_{2} \mathrm{Cl}$ wagging peak at $1267 \mathrm{~cm}^{-1}$, have disappeared from the aminated copolymer spectrums. These absences of characterized peaks attribute to the $-\mathrm{CH}_{2} \mathrm{Cl}$ groups were aminated to the $-\mathrm{NR}_{4}{ }^{+}$. Some new peaks appear at $1486 \mathrm{~cm}-1$ and $1025 \mathrm{~cm}^{-1}$ correspond to asymmetric stretching vibration and bending of $-\mathrm{NR}_{4}{ }^{+}$groups. Moreover, the broad peaks at $3409 \mathrm{~cm}^{-1}$ attribute to the -OH vibration. Compared with the $\mathrm{PE}-\mathrm{g}-\mathrm{PVBC}$ spectrum without styrene addition, the $\mathrm{OH}$ stretching peaks at the $3409 \mathrm{~cm}^{-1}$ are more obvious with the addition of styrene after quaternization. In addition, the stretching and bending at $1486 \mathrm{~cm}^{-1}$ and $1025 \mathrm{~cm}^{-1}$ become more intense after the styrene addition. These indicate that the ideal for adding styrene in the polymerization process is a right direction to increase the $\mathrm{VBC}$ grafted on the PE films. However, the continuedly increase in the amount of styrene would decrease the $\mathrm{VBC}$ amount, resulting in the less functional group grafted. 


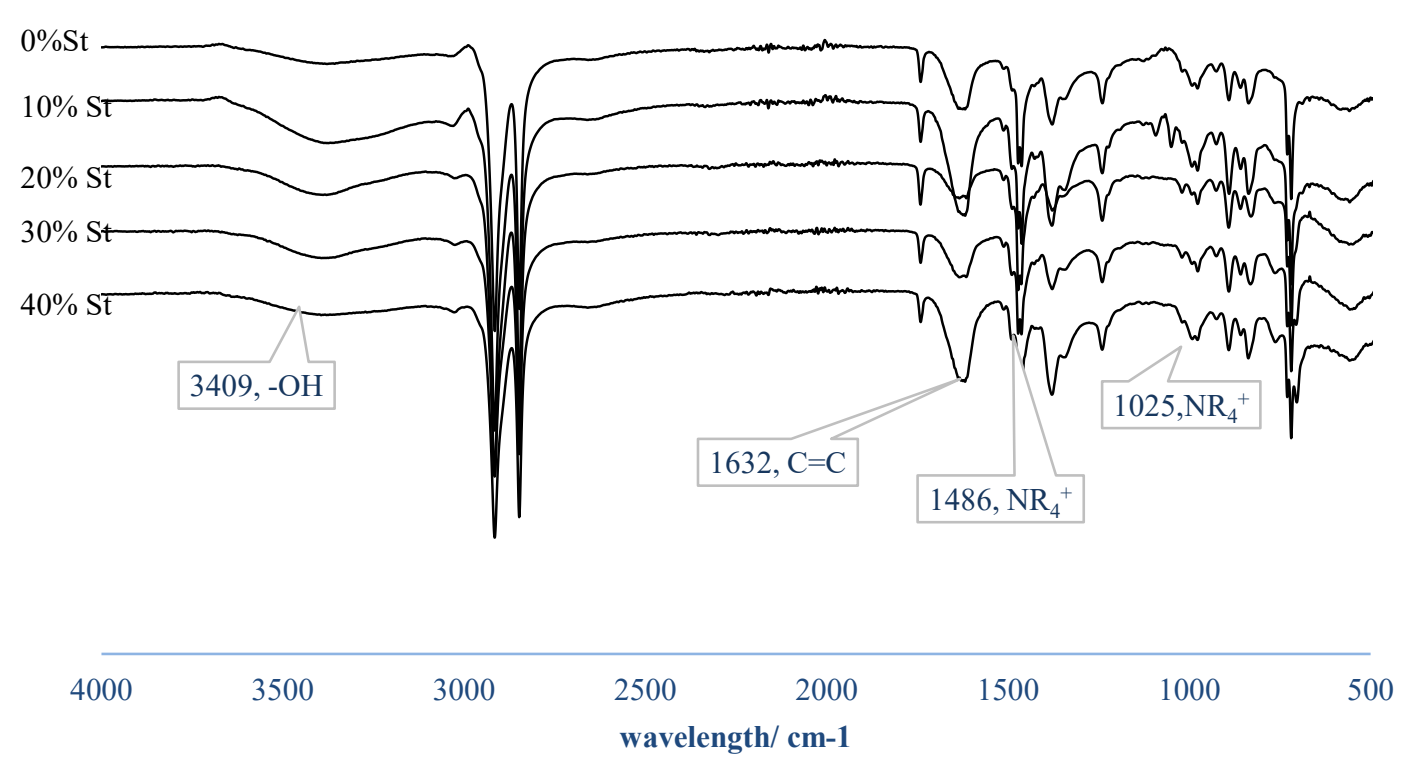

Figure 5.10. FTIR spectra of aminated graft copolymers $\left(\mathrm{OH}^{-}\right.$form) (from top to bottom: St: $\mathrm{VBC}=0: 100,10: 90,20: 80,30: 70,40: 60)$.

\subsubsection{Characterization of AEM by SEM and TEM analysis}

The FE-SEM micrography of poly (ethylene-St-VBC) with various styrene amounts were represented on Figure 5.11. (PS from 0\% to 20\% mass fraction). There are two different phases in these copolymers; one is initial polyethylene material matrix, and another is grafted monomers. The morphology of three membranes present distinctly different with the increasing styrene addition. The grafted polymer PE-g-PVBC without styrene shows obvious phase separation. The irregular dimensional embossments and uneven distributional spherical particle demonstrate the low compatibility between polyethylene and VBC. The appearance of tiny pores dispersed on the matrix indicate the poor miscibility between VBC and PE. With the addition of styrene, the spherical particles with a size of less than $1 \mu \mathrm{m}$ distributed more uniformly on the polyethylene matrix. In addition, the morphological structure of grafted copolymer changed obviously after adding styrene, the tiny pores disappeared. It is speculated that the formation of polymer PE-g-St reduce the interfacial tension between PE and VBC monomers; which leads to the PE based matrix becoming more miscibility with the grafted monomers.

With the increase in styrene, the surface of the PE matrix is observed to be more smooth and dispersed phases became more continuous. On the copolymer surface appeared more 
spherical particles of less than $1 \mu \mathrm{m}$, indicating the grafted monomers styrene and VBC were uniformly distributed on the PE matrix surface. It is concluded by TauqirA. Sherazi that the phase separation could lead to interruption in the ions conducting channels, resulting in the observed increase in resistance of ion exchange membranes [60]. The uniform phase facilitates the ions conduction and exchange.
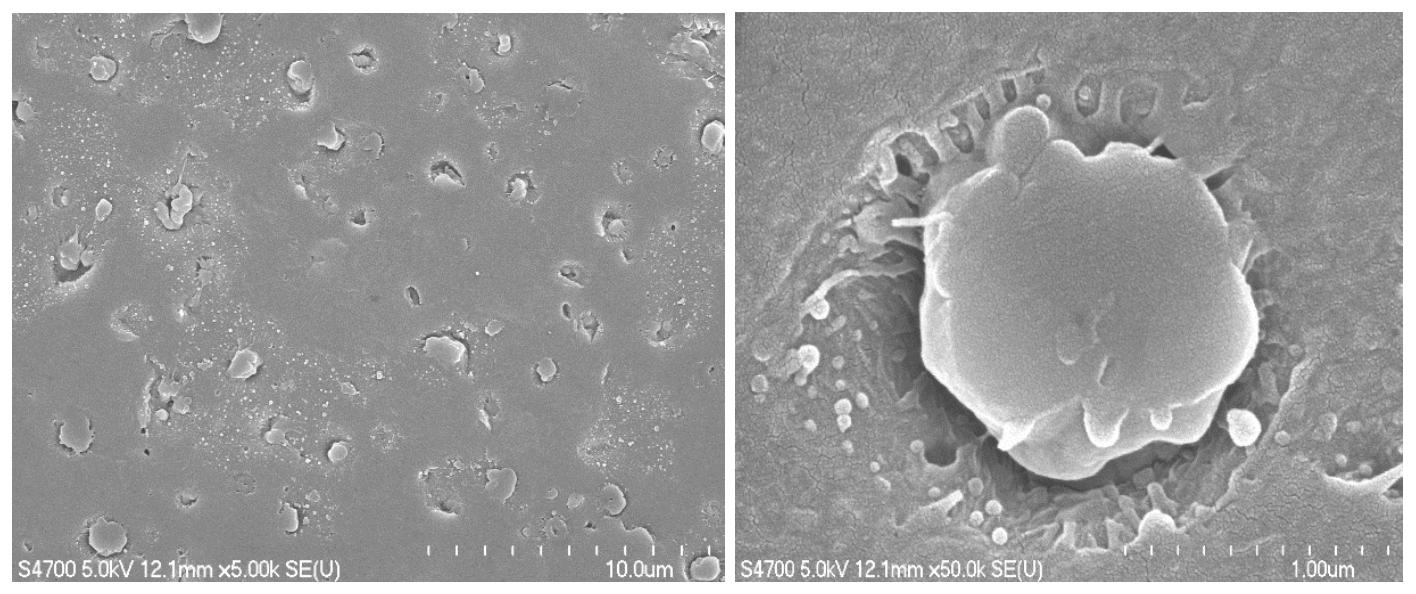

a

b

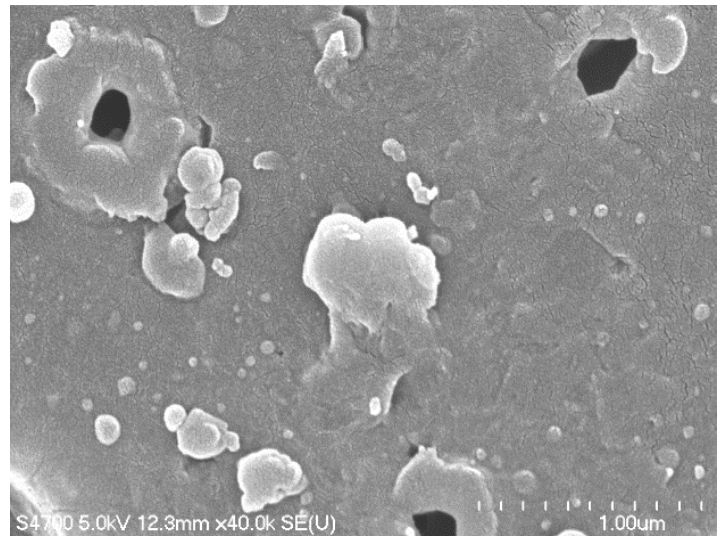

c

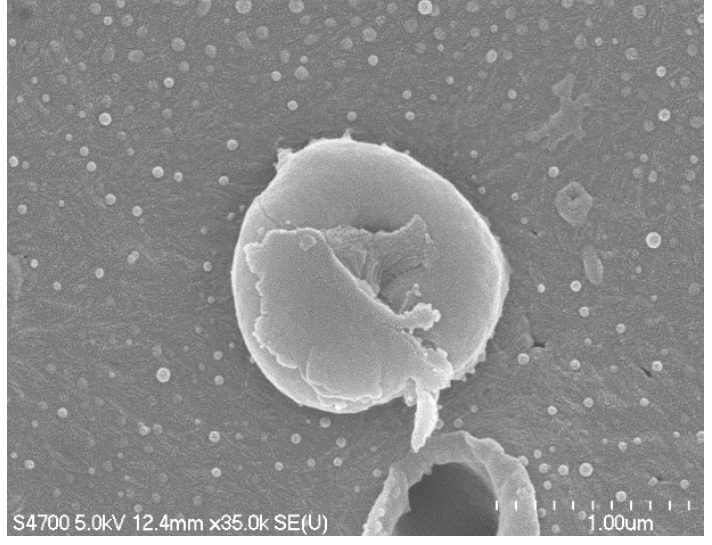

d

Figure 5.11. SEM images of (a)(b) PE-graft-P(VBC) and (c) PE-graft-P(St-co-VBC) (St: $\mathrm{VBC}=10: 90)$ copolymer(d) PE-graft-P(St-co-VBC) $(\mathrm{St}: \mathrm{VBC}=20: 80)$

In Fig. 5.12. the sample image covered by microparticles composited with polyethylene, PE-g-PVBC or PE-St-PVBC. The microparticles represented by bright agglomerates have less molecular weight and shorter polymer chains than the dark agglomerates. The agglomerates are generally larger in copolymer without adding styrene than those with 
$10 \% \mathrm{St}$ added. Furthermore, the bright and dark agglomerates have more obvious separation in fig 4(a) than those in fig.4 (b). This indicates the addition of St improve the miscibility of PE and VBC. This is consistent with SEM observations.
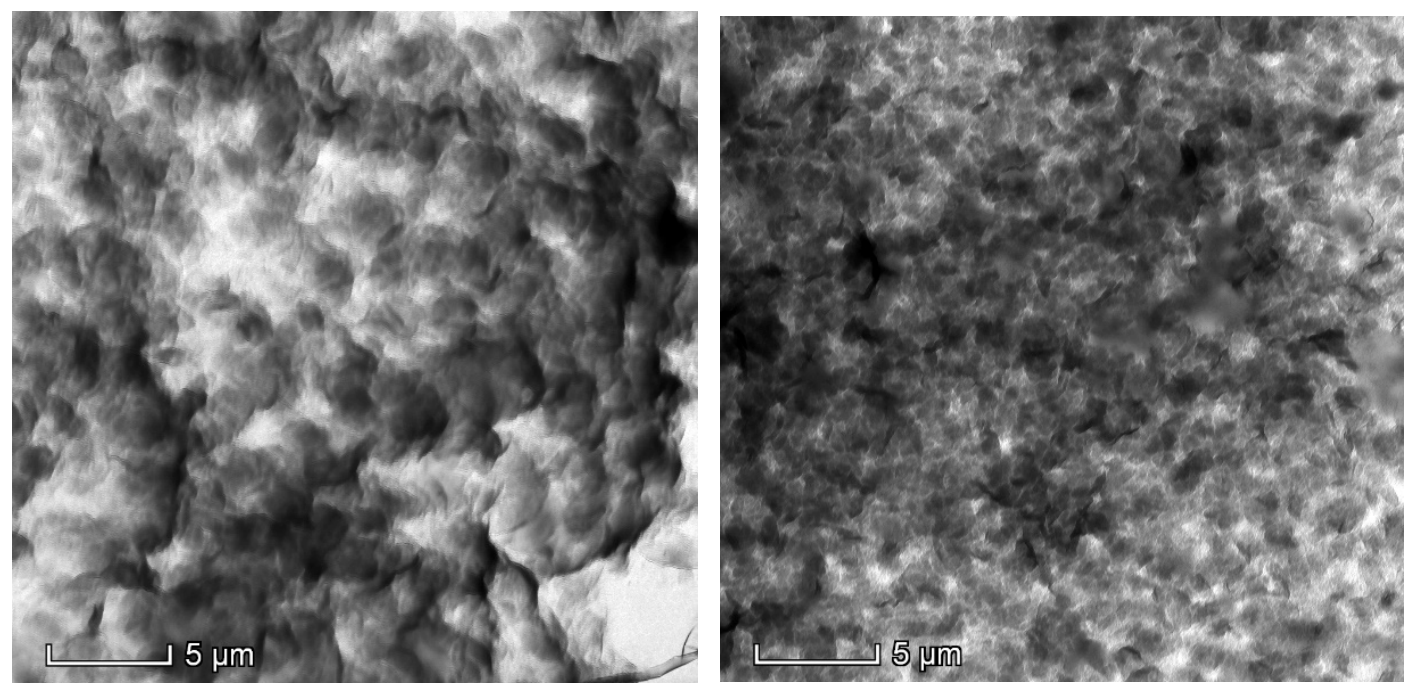

Figure 5.12. TEM images of (a) PE-g-PVBC and (b) PE-St-PVBC) (St: VBC=10:90) copolymer

\subsubsection{Ion exchange capacity (IEC)}

The poly(ethylene-St-VBC) membrane was treated by ammonization to form ionic functional group $-\mathrm{NH}^{3+} \mathrm{Cl}$ for ion exchange capacity (IEC) measurement. IEC is defined the number of exchangeable ionic groups per weight on the membrane in aqueous solution, and it can be determined though back titration to $\mathrm{Cl}^{-}$in this case. Figure 5.13 shows the influence of the addition of styrene to the IEC of AEM. The plot of IEC values from titration shows the presence of styrene greatly enhance the IEC of AEM, implying that more functional groups were introduced to the PE branch chains. The ion exchange capacity of $10 \%, 20 \%$ and $30 \%$ styrene monomers addition ratios are all higher than that found with AEM without styrene addition. The peak value of IEC appears at $10 \%$ styrene added, the value is $1.26 \mathrm{mmol} / \mathrm{g}$, which is $57 \%$ higher than IEC of non-styrene AEM.

Figure 5.13. also shows the IEC values determined by elemental analysis with calculating $\mathrm{N}$ content are higher than the IEC values calculated by titrating $\mathrm{Cl}$-. The IEC values from elementary analysis are close to the theoretical values that were calculated when assuming $100 \% \mathrm{Cl}$-attachment on the functional group would be used for ion exchanging. Whereas the IEC values from titration can reflect a practical ion exchange situation. Continually increasing the amount of styrene results the IEC decreasing after peak value due to the VBC amount decreasing. 
However, the continued increase in the amount of styrene would decrease the VBC amount, resulting in the IEC decreased. In other word, the functional groups being attached with exchanged ions though quaternization come from $\mathrm{VBC}$, other than styrene. The goal of adding styrene is to make more VBC grafted on the PE matrix, but it does not contribute to bring functional groups by itself.

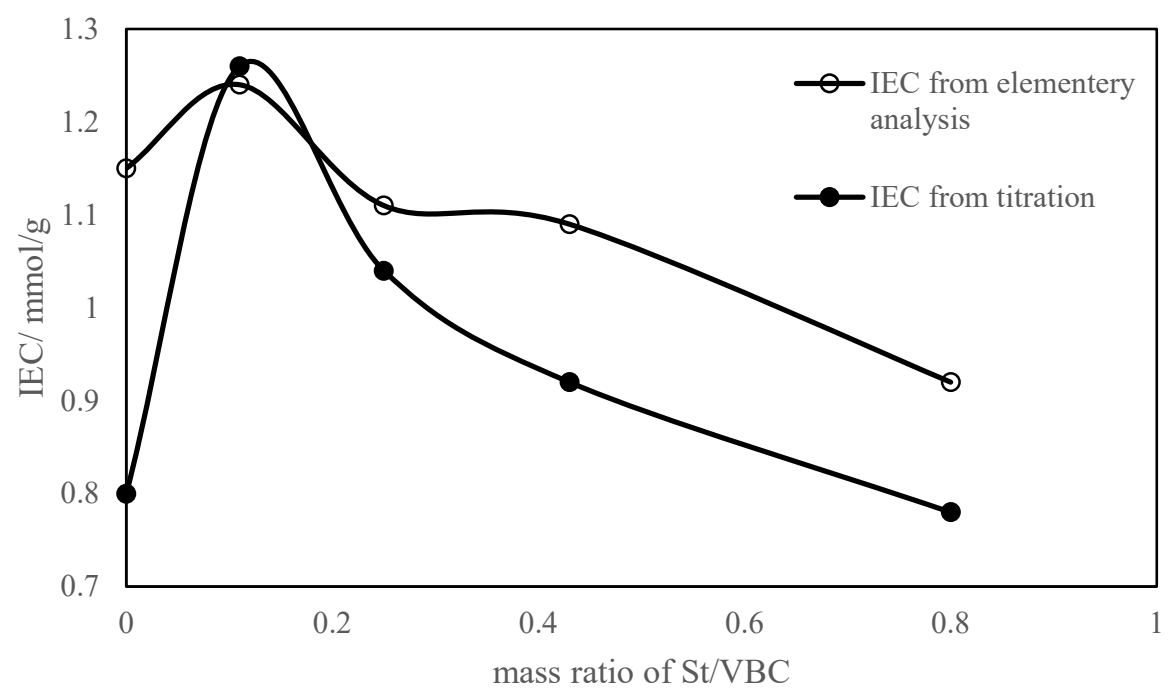

Figure 5.13. Effect of St on the IEC of anion exchange membranes $\left(\mathrm{OH}^{-}\right.$form).

\subsubsection{Water uptake and membrane swelling ratio}

Water uptake is a measurement of the amount of water absorption in the membrane, in terms of wt $\%$. High water uptake contributes to the ions transportation and would lead to higher ion conductivity. A membrane with a high IEC tends to absorb more water, but excess water uptake will lead to undesirable dimension deformation (swelling or shrinking) and structural instability. When membranes are fixed on the electrochemical device, the excessive water uptake would result in high water penetration between the cation and anion exchange membrane, which may decrease treatment efficiency for water concentration. A good balance between IEC and water uptake is usually seriously considered and designed.

The water uptake and membrane swelling property of AEM with various styrene proportions are represented in Figure 5.14. Apparently, the AEM (10\% styrene proportion) with the highest IEC value uptakes the most water amount and produces the highest membrane dimensional deformation, which is in accord with the prediction. The high-water uptake is mainly due to the high hydrophilic functional groups grated on the membranes which are associated on the IEC. Compared with commercial membranes, 
such as Nafion ${ }^{\circledR} 115$ ( $236 \%$ water uptake and 1.4 IEC value) the AEM with $10 \%$ and $20 \%$ proportion both have acceptable IEC values and water uptake rates, and the dimension variation are relative stable.

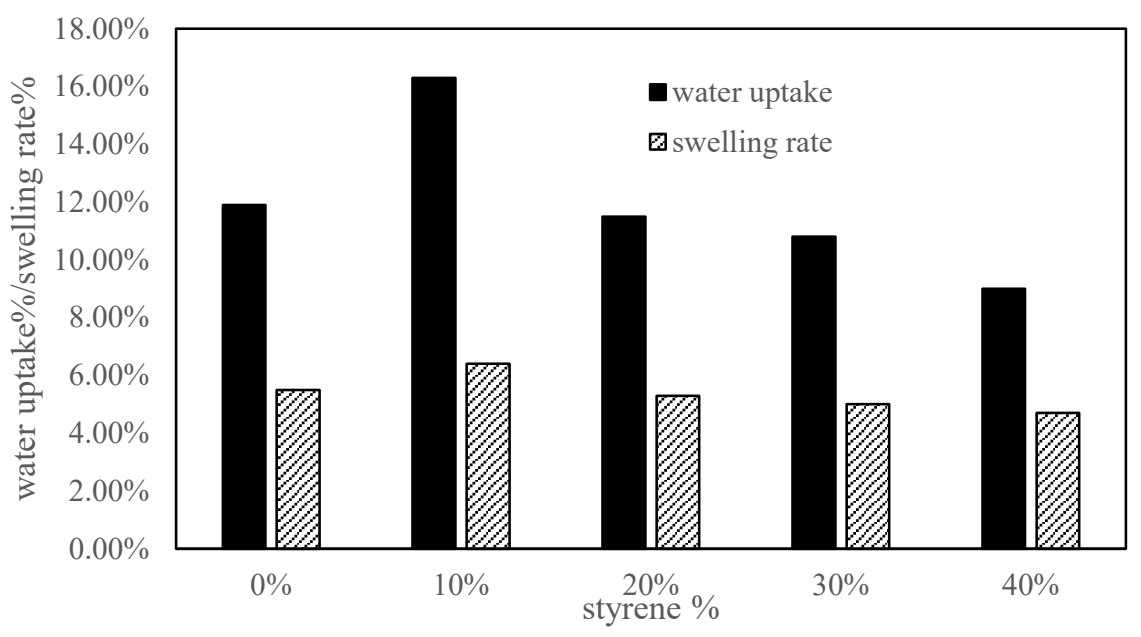

Figure 5.14. Effect of styrene addition on water uptake and swelling rate of AEM

\subsubsection{Comparation of working performance for AEM with and without styrene}

The comparation of working performance for commercial AEM, PE-g-PS and PE-StPVBC AEM were presented in Figure 5.15. Six PE-g-PS and PE-St-PVBC AEMs were cut into shape and fitted into the electrodialysis machine with seven pieces of Japan imported CEM respectively. Compare to the commercial AEM, both synthesized AEM have less change rate of conductivity for concentrated solution and dilute solution, But PE-St-VBC based AEM have sharper slop than the curves of PE-g-VBC AEM, which indicate the PE-St-PVBC based AEM has higher ions processing efficiency. This suggests adding St to VBC monomer can assist VBC to be grafted onto PE molecules. Compared with commercial products from Japan, our anion membrane has lower efficiency (defined as current (conductivity-initial conductivity)/initial conductivity) (Figure 5.16). The efficiency of PE-St-PVBC based AEM is superior to PE-g-VBC based AEM. 


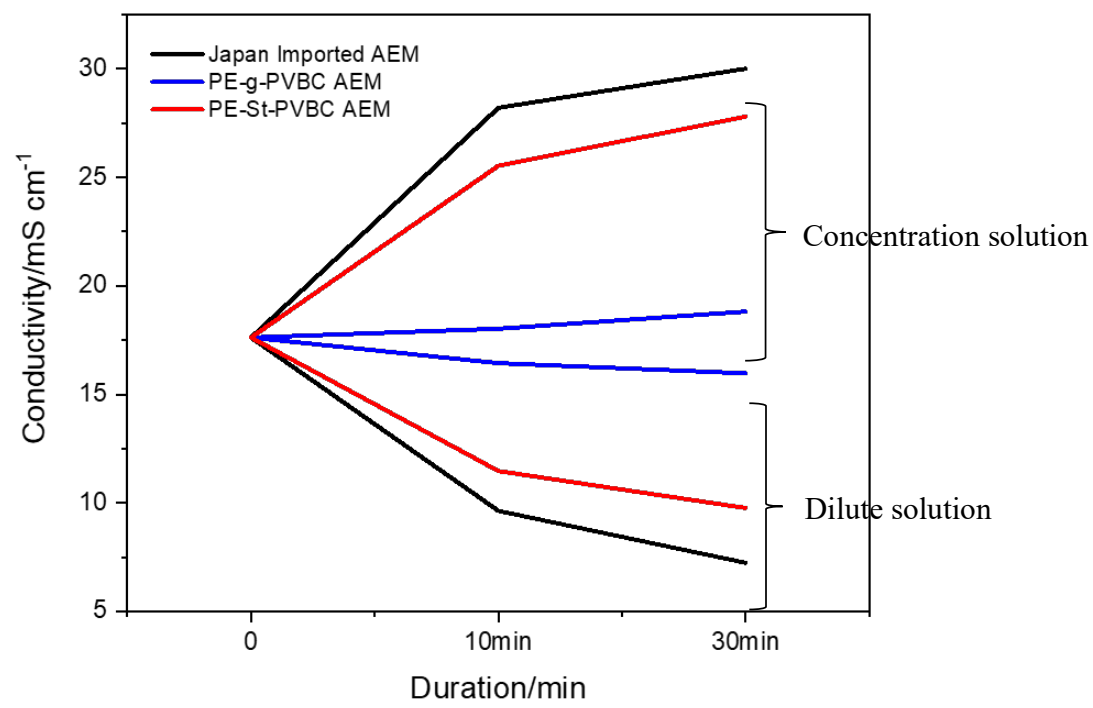

Figure 5.15. Comparation of commercial AEM, PE-g-VBC based AEM and PE-St-VBC based AEM

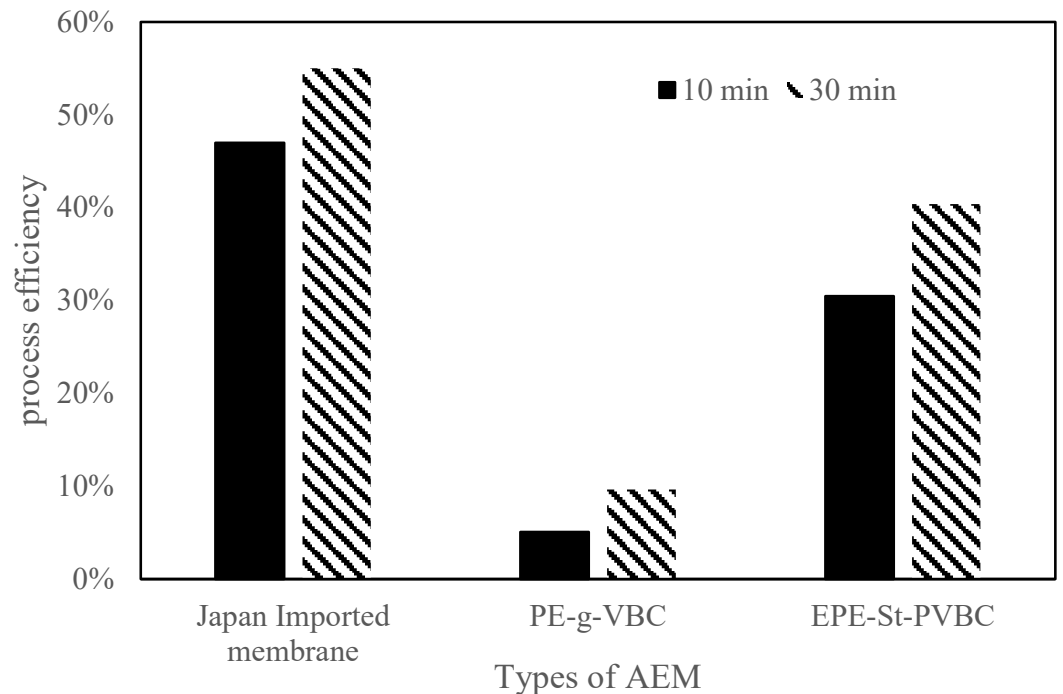

Figure 5.16 Comparation of processing efficiency for different AEM types

\subsection{Summary}

Compare to the traditional method to synthesize AEM, the method of chemically grafting $\mathrm{VBC}$ on PE followed by quaternization and alkalization is a low risk and advanced way to synthesize AEM. The later can obtain more quaternary amine groups on AEM but the IEC do not reach the expectation. It was proved the temperature and duration of 
quaternization are not the reason for insufficient quaternary amine groups grafted. The St addition has a positive effect on the final properties of the AEM. The 10\% St addition dramatically improves the IEC property. The value is $1.26 \mathrm{mmol} / \mathrm{g}, 57 \%$ higher than IEC of non-styrene AEM. Compared with the commercial membranes with $36 \%$ water uptake and 1.4 IEC value, the PE-St-PVBC based AEM with 10\% and 20\% St both have acceptable IEC values and water uptake rate, and the dimension variation are relative stable. We also found that further addition of St decreased the grafting degree of VBC since St can compete with VBC in graft polymerization. The final membrane was characterized by elemental analysis, FTIR, SEM and TEM. Compared with the PE-gVBC spectrum without styrene addition, the $\mathrm{OH}^{-}$stretching are more obvious with styrene addition after quaternization. SEM and TEM indicated a clear phase separation between the grafting phase and the PE matrix on PE-g-PVBC without St addition. 


\section{Studies on glass fiber reinforced poly(ethylene- grafted-styrene) based cation exchange membrane composite}

\subsection{Introduction}

Over the past decades, there is renewed interest in developing high-performance ion exchange membranes, which play important roles in various industrial applications, such as fuel cell, desalination and wastewater treatment $[14,45,96,101]$. Typically, ion exchange membranes are composed of inert polymers such as polyethylene, polyvinylidene fluoride and polyvinylchloride [4,7] and reactive polymers such as polystyrene and polysulfone that can be functionalized by ionic groups $[8,10]$. The desired properties of ion exchange membranes are determined by the inert backbone polymers, reactive polymers and methods to combine them.

According to the connecting way of charged groups on the matrix, ion exchange membranes can be classified into homogeneous and heterogeneous membranes. Homogeneous ion exchange membranes have wide applications due to the excellent electrochemical performance [7]. However, during the long service life, homogenous ion exchange membranes cannot maintain the desirable mechanical properties and structural stability in the harsh environment, such as plating wastewater, which requires the high structural and chemical stability for the membranes [13]. The studies of improving mechanical properties and microstructure stability of ion exchange membranes have attracted much attention in recent years. Mechanical property is a key factor for ion exchange membrane in electrodialysis (ED) stack application. Although the ED is not a pressure driven process, the membrane needs to resist some small overpressure during operation.

Glass fiber (GF) reinforced polymer composite gradually became a competitive structural material due to the excellent mechanical properties. Three factors affect the fiberreinforced composite's mechanical properties: intrinsic properties of matrix materials, the strength of interfacial chemical bonding between fillers and matrix, and the load transfer efficiency of interphase [103]. Many researches indicated that the addition of GF only improved composites' mechanical properties at a low extent if no modifier treats to GF [102][103]. This is due to the insertion of GF destroys the phase morphology of the matrix. GF was also reported to lower impact resistance and load transferability of the fiber-reinforced composite due to hydrophilic nature of hydroxyl groups. The reason behind that is there is no chemical bonds existed between GF and matrix.

Recently, many methods have been studied to improve the interfacial adhesion and construct chemical bonds between fibers and matrix. The styrene/2-

ethylhexylacrylate/divinylbenzene solid foams was reported to be reinforced by the 
sonicated silica particle up to 3\%. The sonication treated silica particle considerably enhanced the composites' Young's modulus and crush strength [104]. Zhang etc. reported the mechanical properties of long glass fiber-reinforced polypropylene (PP) improved using dicumyl peroxide and maleic anhydride as adhesion promoters to increase interfacial interaction between PP and GF. This research obtained a result that the content of dual compatibilizer could influence the composite's strengthening extent [105]. Nano-silica treated three kinds of silane coupling agents, including $\gamma$-aminopropyltriethoxysilane (KH550), Vinyl-triethoxysilane (A-151), and $\gamma$-chloro-propyl trimethoxy silane (A-143), were reported to modify GF and could effectively improve the composite's mechanical properties [102]. This study also concluded that the coupling agent's content affected GF reinforced composite's mechanical properties enhancement. The $\gamma$-(methacryloyloxyethyl) trimethoxy silane has been reported to treat the GF and lead to a vinyl functionalized GF surface, which served to covalently bond between styrene/divinylbenzene copolymer and GF [107]. Other interfacial adhesion strengthening methods were studied including introducing adhesion prompters to increase the compatibility between fiber and matrix [108][109]. Compatibilizers such as admicellar, maleated ethylene and a few acrylic acid copolymers were also reported to enhance the adhesion between the fiber and matrix [108]. In above studies, treating GF with the adhesion promoter and compatibilizer are promising way to improve interfacial adhesion between GF and matrix.

The idea of improving interfacial adhesion between the fiber and polymeric matrix by modifying the fiber surface can also be introduced to GF and ion exchange membrane composite. In Křxčíks work, the polypropylene and GF were used as low-cost alternative to common woven fabric in ion exchange membrane [110]. He confirmed that short polypropylene fibers rapidly increased the mechanical strength in machine direction. Minna Annala ect. reported the storage modulus and loss modulus of sulfonated ethylene/styrene copolymer were improved moderately after adding GF [111]. The strengthening effect of GF on composite was confined without surface modification. However, the studies of improving the mechanical property of GF/ion exchange membrane composite through modifying GF's surface have not been widely concerned. Most studies focused on even dispersing GF on matrix, rather than improving interfacial adhesion, such as the above two examples. The issue is the introduction of the higher modulus GF leads the increase in tensile strength but destroy the phase morphology of matrix [99]. The damage of matrix's consistency would result in the resistance to the composite strengthening. In our study, four coupling agents were chosen as surface modifiers to improve the interfacial adhesion. Besides the silane surface modifiers (3(Methacryloxy)propyl] trimethoxy silane (3-MPS), 1,6-bis (trimethoxysilyl) hexane (1,6bis), and Triethoxyvinylsilane (TES)), the compatibilizer (Poly(propylene-graft-maleic anhydride) (PP-g-MA)) was also chosen to study the treatment effect on GF surface. TES treated GF was reported to improve the composite's mechanical properties effectively [112]. But the GF was not directly treated by TES. It was combined with nano-silica to modify GF. PP-g-MA was reported to be combined with two organofunctional silanes to 
increase the interfacial adhesion in glass fiber-polypropylene (PP) reinforced composites [113]. The 3-MPS and 1,6 bis contained one and two trimethoxy respectively, the characteristic functional group in many coupling agents for GF modification, were chosen to study the treatment effects due to analogous organofunctional structure.

The objective of this article is to investigate the modifying effects of different coupling agents on GF, which were used to strengthen poly(ethylene-co-styrene) based cation exchange membrane. The mechanical properties, morphology characterization, and FTIR of GF reinforced poly(ethylene-grafted-styrene) based cation exchange membrane (CEM) composites were studied in this paper. To assure the mechanical properties enhancement is not at the cost of other membrane properties, the ion exchange capacity and water uptake ability were also investigated in this paper.

\subsection{Experimental results and discussions}

\subsubsection{The effect of various coupling agents on mechanical properties of CEMs composite}

The effects of various coupling agents on mechanical properties of GF/CEM composite are illustrated in Figure 6.1-6.2. The pure membrane specimen is the membrane without GF. The blank sample is the ion exchange membrane with untreated GF. Compare to the virgin membrane and blank sample, the tensile strength of composites with GF treated by 1,6 bis, 3-MPS and PP-g-MA all increased. The sample treated by 3-MPS obtained significant stress enhancement. All the samples with treated GF have higher tensile modulus. It's also reflected by the slopes of stress-strain curves in Figure 6.2. The steeper the slope, the higher the tensile modulus. The improved tensile strength and tensile modulus results indicate that with the GF's addition, the CEMs composites would not stretch easily and have better deformation resistance against water pressure. However, the strain value decreased when adding GF fillers in CEM, especially when adding the GF treated by coupling agents. Those results indicate that the GF/CEM composites become strong but not tough with modified GF. The composite with modified GF needs more force to be broken but cannot suffer higher elongation. 


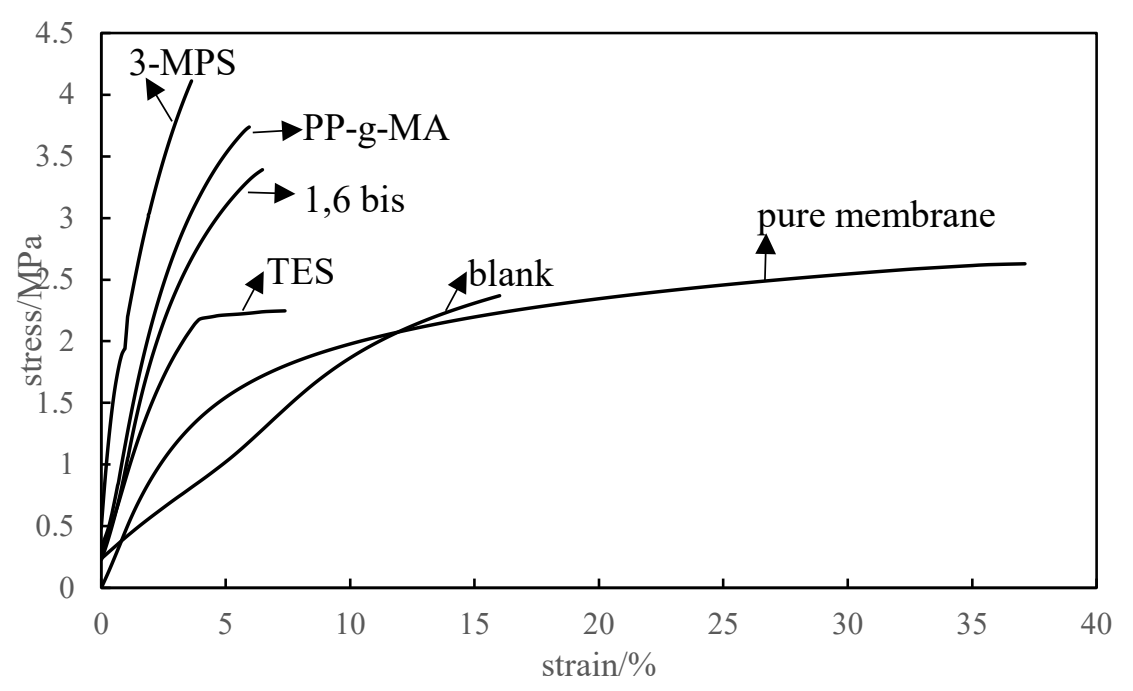

Figure 6.1. Stress-strain relations of CEM composite with different modified GF

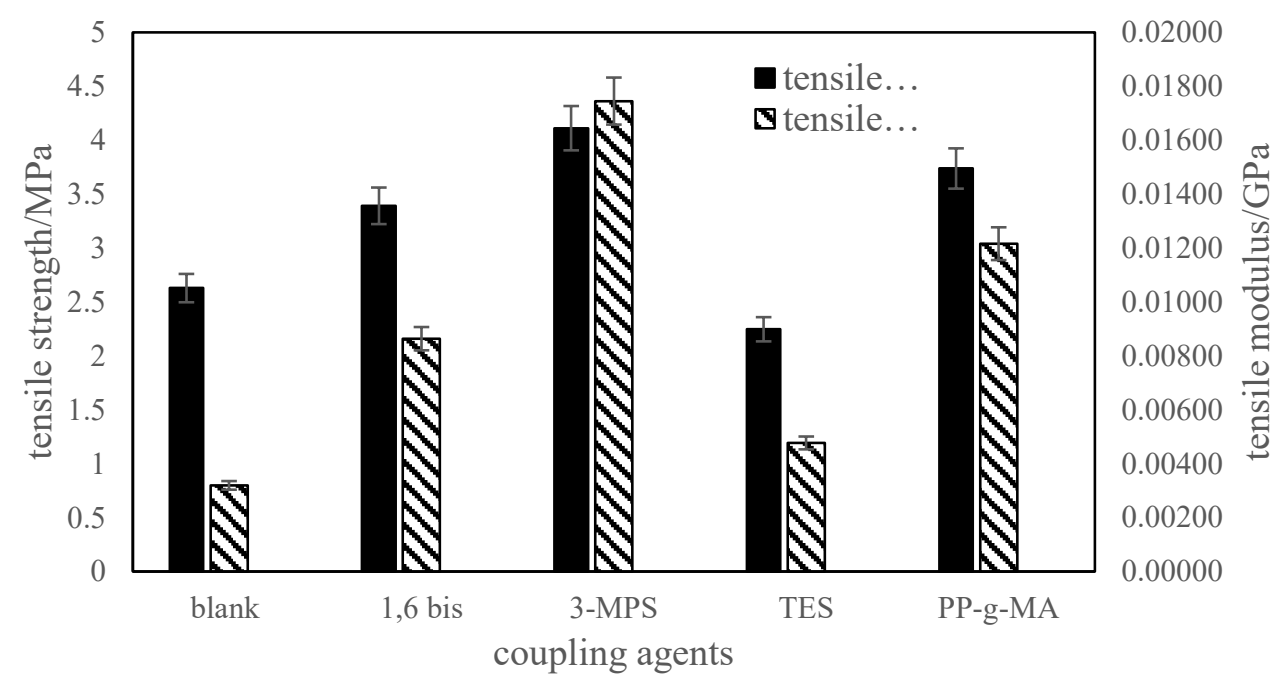

(a)

Figure 6.2. Tensile strength and tensile modulus of CEMs composite with different modified GF

Not only the coupling agents' types affect composites' mechanical strength, but also the coupling agent's concentrations. Since the 3-MPS showed superior modification effect on GF, the 3-MPS modified GF was chosen to be investigated the effect of coupling agent concentration on mechanical properties. Figure 6.3. exhibits the stress-strain relationship of CEM composites with the GF treated by increasing 3-MPS concentration. The results show the 2\% 3-MPS concentration has a superior treating effect on CEM composite's 
stress enhancement. However, the strains weaken gradually by increasing 3-MPS concentration. Figure 6.4. shows the tensile strength and tensile modulus of GF/CEM composite increase until the 3-MPS' concentration reached $2 \%$. After that, the tensile strength and tensile modulus both decrease with the increasing 3-MPS' concentration treated on GF.

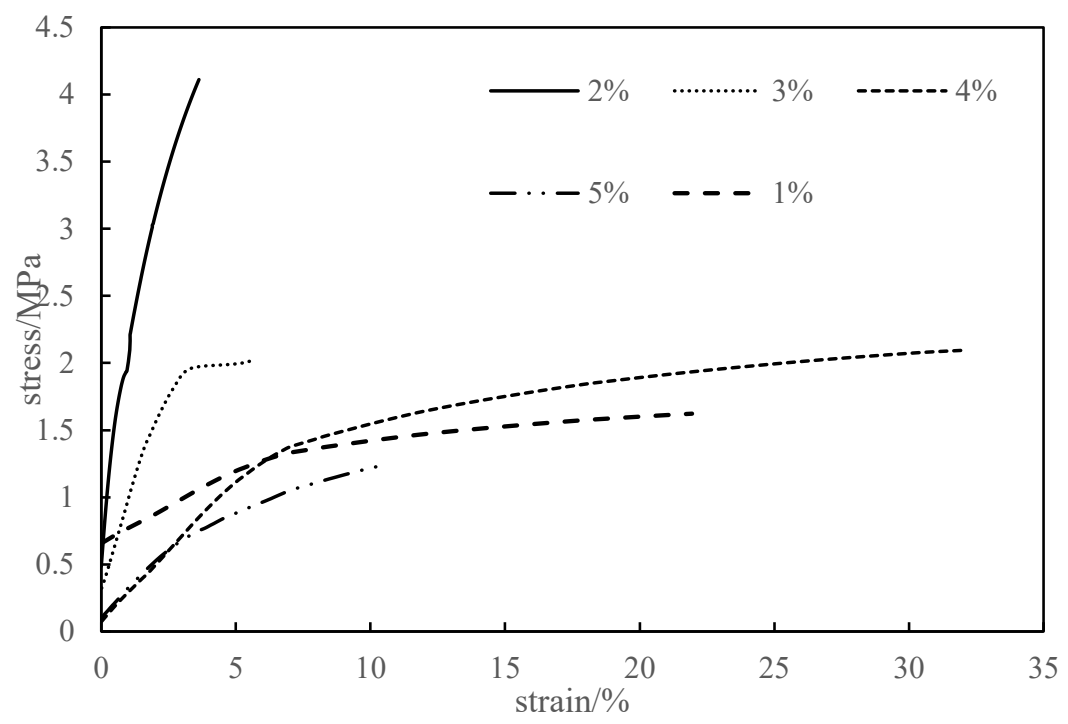

Figure 6.3. Stress-strain relationship of CEM composite with GF treated by varying 3MPS concentration.

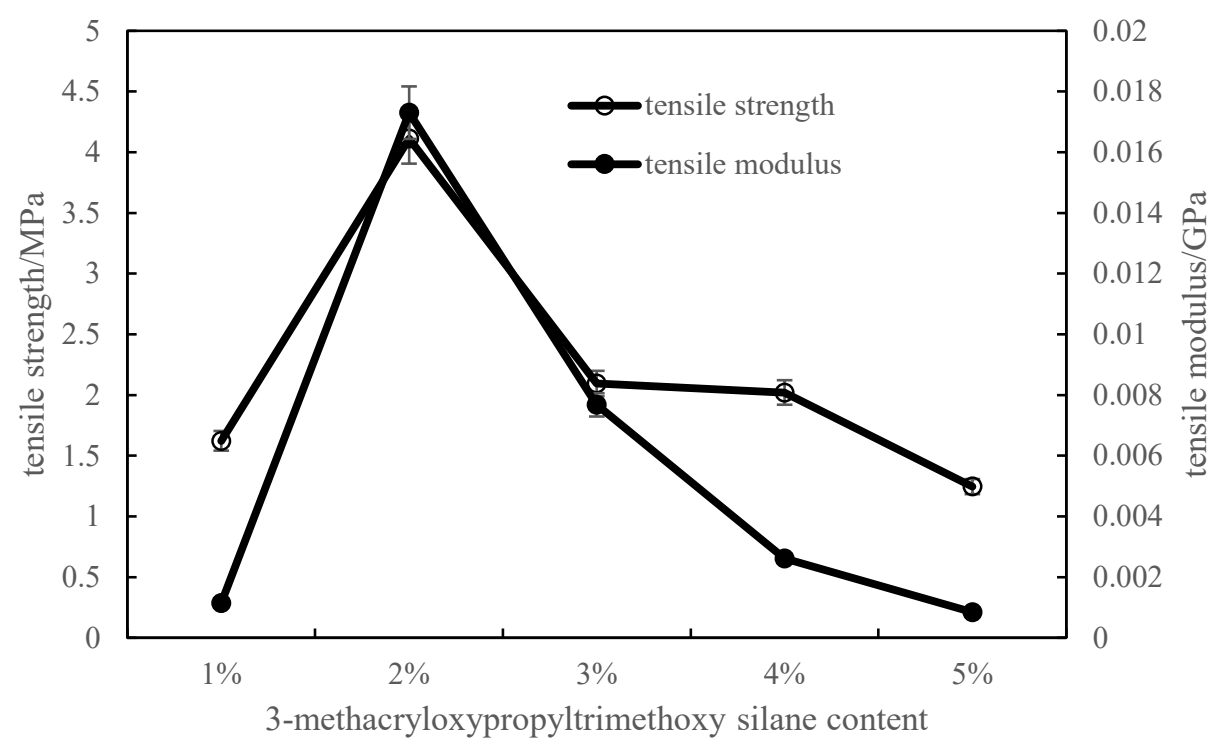


Figure 6.4 Tensile strength and tensile modulus of CEM composite with GF treated by varying 3-MPS concentration.

\subsubsection{The effect of various coupling agents on dynamic mechanical property of IEMs composite}

The dynamic mechanical property can be evaluated by storage modulus and loss modulus. The storage modulus reflects the ability of materials storing energy elastically, representing the elastic portion. The loss modulus reflects the ability of materials dissipating stress through heat, representing the viscous portion. The loss modulus ratio to storage modulus in a viscoelastic material is defined as the tan delta, which provides a measure of damping in the material.

The influences of various coupling agents on storage modulus as a function of temperature are presented in Figure 6.5. It is found that the storage modulus of GF/CEM composites with 1,6 bis, 3-MPS, PP-g-MA modified GF are all improved compared to the blank sample and pure membrane, which explains why the tensile strength and tensile modulus enhanced. This effect is more noticeable when the temperature is below $120{ }^{\circ} \mathrm{C}$, which attribute to interfacial chains between GF and matrix become soft when the temperature is high [109]. The unmodified GF, which means no adhesive between the GF and matrix, results in the negative influence on storage modulus at the higher temperature. This negative influence is due to the membrane's integrity was destroyed by GF insertion. The GF treated by TES is not shown the positive effect on composite, resulting in lower storage modulus when the temperature is below $70{ }^{\circ} \mathrm{C}$.

The loss factor $(\tan \delta$ ) -temperature curves in Figure 6.6. have two bumps corresponding to two transitions. The first transition happened around $65-70{ }^{\circ} \mathrm{C}$, corresponding to the glassy-rubbery transition. The second transition, which occurred about $120-135{ }^{\circ} \mathrm{C}$, is associated with crystallites transformation. The crystallites transformation represents the interface adhesion status of composite material. If briefly compare with the blank sample, the crystallites transformation bumps of CEM composites with modified GF shifts to a higher temperature range. This shift reveals the interfacial adhesion between matrix and GF have been improved after being treated by coupling agents. The tan delta also enhanced after GF modification, indicating the ability of CEM composite dissipating stress has been improved. The fillers' interfacial adhesive effects on dynamic mechanical properties can be accurately expressed by Luis Ibrarra's formula [110]. This formula (6.1) assumes that the composite loss factor is the sum of component lass factor and volume fraction products.

$$
\mathrm{A}=\frac{1}{1-V_{f}} \times \frac{\tan \delta c}{\tan \delta m}-1
$$


$\tan \delta \mathrm{c}$ and $\tan \delta \mathrm{m}$ represent the loss factors for composite and matrix respectively. Vf represents the volume fraction of glass fibers. The parameter $\mathrm{A}$ is introduced to measure the adhesive effects between fillers and matrix, $\mathrm{A}$ is defined as eq. (6.2):

$$
A=\frac{V_{i}}{1-V_{f}} \times \frac{\tan \delta_{i}}{\tan \delta_{m}}
$$

Assuming $\tan \delta \mathrm{f}=0$, the interfacial volume fraction $\mathrm{Vi}$ is ignored, then eq. ((6.2) can be rewritten as followseq. (6.3):

$$
\frac{\tan \delta_{c}}{\tan \delta_{m}}=\left(1-V_{f}\right)(1+A)
$$

Hence, the parameter A can be rewritten as Luis Ibrarra's formula (eq. (6.4)):

$$
A=\frac{1}{1-V_{f}} \times \frac{\tan \delta c}{\tan \delta m}-1
$$

The $\mathrm{A}$ is an inverse proportion to the interfacial adhesion. Table 6.1. shows the A values and the corresponding $\tan \delta \mathrm{c}$ and $\tan \delta \mathrm{m}$. The A values of GF/CEM composites with GF treated by 3-MPS and PP-g-MA are relatively lower than others, indicating higher glass fiber-matrix adhesive effects obtained at their interface. 3-MPS and PP-g-MA were also demonstrated have better modification effects on improving the adhesive capacity of the interface. The negative value of A in the blank sample represents the addition of raw GF impaired interface adhesion. The applied force could not dissipate from the matrix to GF.

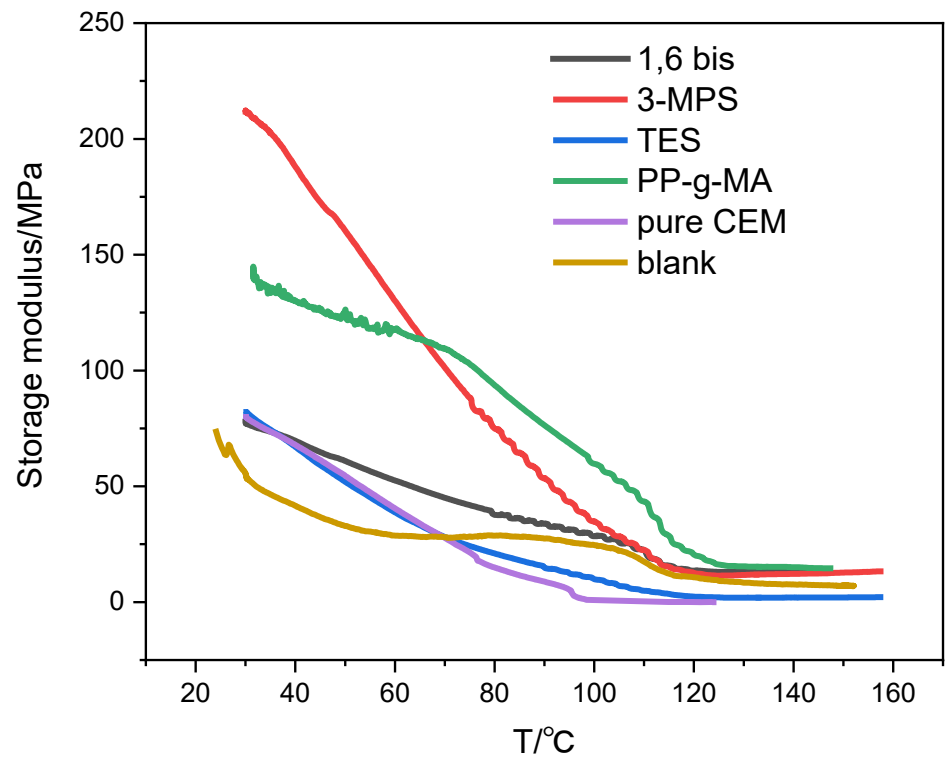

Figure 6.5. Storage-temperature relationship of the composite with different coupling agents treated GF. 


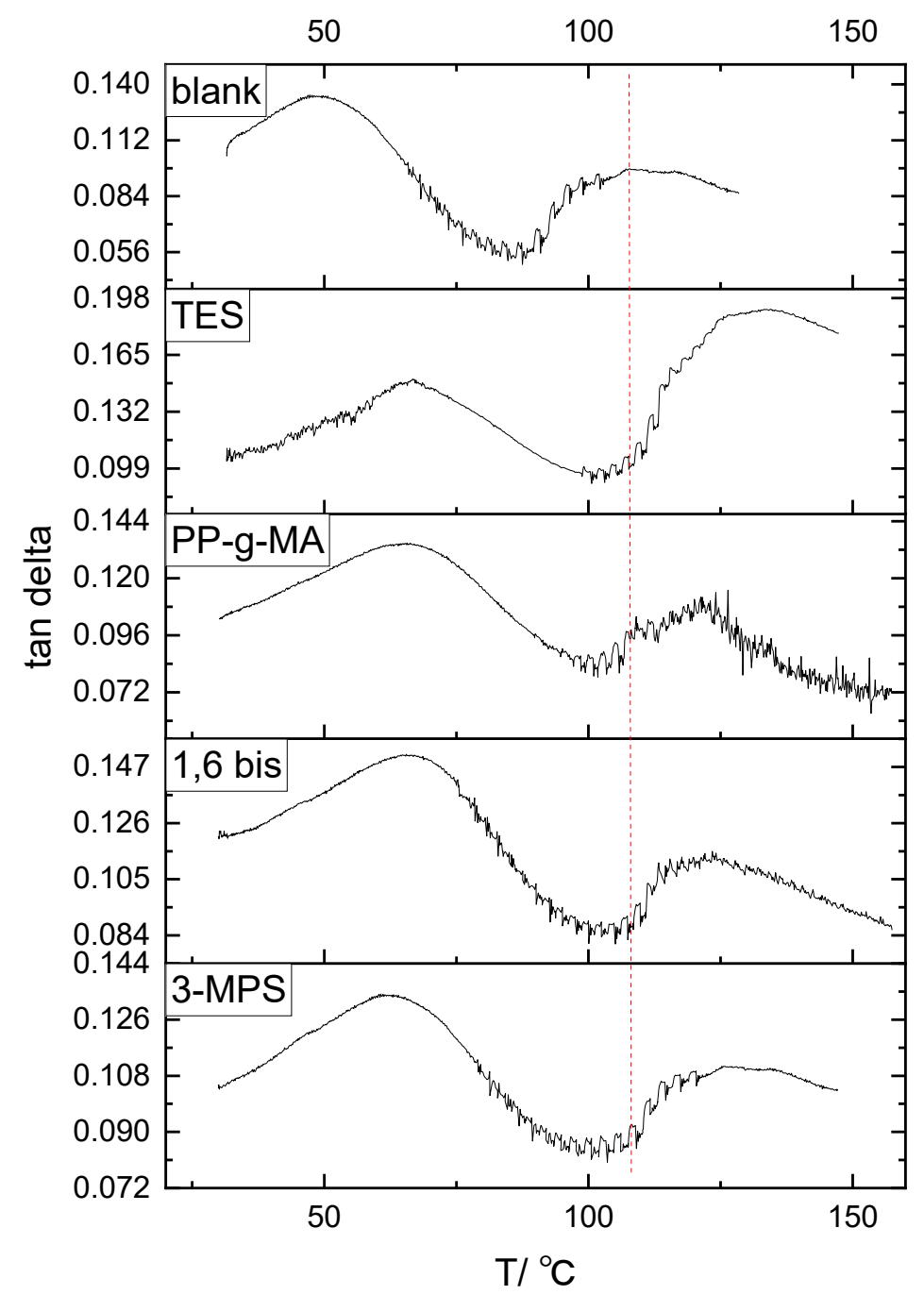

Figure 6.6. Tan delta-temperature relationship of coupling agents with different coupling agents treated GF.

Table 6.1. Glass fiber surface treatment coupling agents on the adhesion of the interface.

\begin{tabular}{cccc} 
Sample & $\tan \delta_{\mathrm{c}}$ & $\tan \boldsymbol{\delta}_{\mathrm{m}}$ & $\mathbf{A}$ \\
\hline Blank & 0.097 & 0.1269 & -0.11118 \\
\hline 1,6 bis & 0.1112 & 0.1269 & 0.01893 \\
\hline 3-MPS & 0.1107 & 0.1269 & 0.01435 \\
\hline TES & 0.1902 & 0.1269 & 0.74281 \\
\hline
\end{tabular}


The effects of 3-MPS's concentration on DMA as a function of temperature are shown in Figure 6.7. The composite with GF treated by $1 \% 3$-MPS has the lowest storage modulus value in the temperature range $25-150{ }^{\circ} \mathrm{C}$ due to the insufficient modification effect. The $2 \% 3$-MPS has the best modification effect on GF, making GF/CEM composite obtain the largest storage modules. However, continually increasing the 3-MPS weaken this enhancement effect. The tensile stress and tensile modulus of composite with 3-MPS treated GF have the same trend. A possible reason would be that the 2\% 3-MPS concentration was enough to react with Si-OH on GF. The higher 3-MPS concentration would lead to GF's surface covered by the redundant coupling agents, decreasing the GF and membrane matrix's interfacial adhesion.

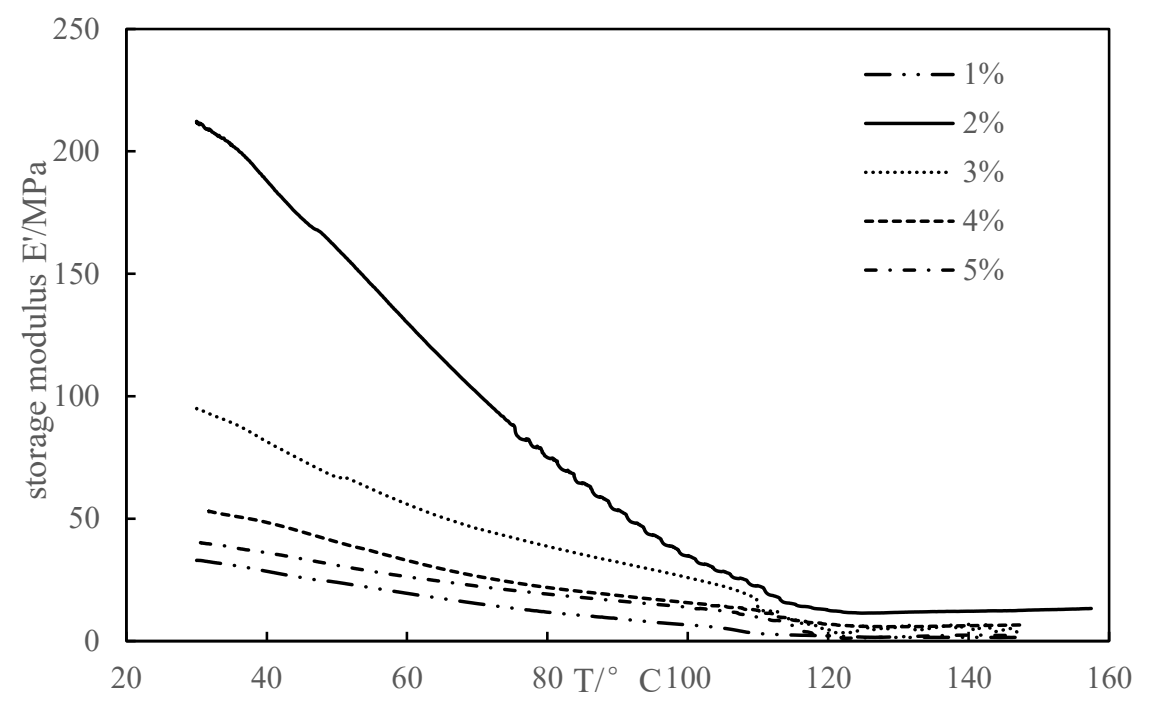

Figure 6.7. Storage modulus-temperature relationship of the composite with increasing 3MPS treated GF.

\subsubsection{CEMs composite morphology analysis}

The surface morphologies of CEM composites with untreated GF and GF treated by four coupling agents were investigated by FE-SEM (as shown in Figure 6.8. and 6.9.). Figure 6.8. a) shows that most of GF are covered under the membrane matrix, but some single glass fibers are dispersed out of the matrix. Besides, many holes appear in the matrix due to the GF insertion. Figure 6.8. b) shows the GF's surface is smooth, and the cavity around GF is loose. It revealed that the interfacial adhesion was inferior due to the lack of chemical bonds between GF and matrix. This weak adhesion results in composites' low 
mechanical properties, and the holes caused by GF insertion also destroy membrane consistency.

Compare to the untreated GF composite, the morphologies of CEMs composites with the pretreated GF present in Figure 6.9. a, c, e and g, showing that the amounts of entwined matrixes on GF's surface increase. Figure $10 \mathrm{~b}$ shows fibers' surfaces become rough and have some thin cross-layer distributed to surface modification. Figure 6.9. b) show that the GFs are wrapped by the polymer colloid layer completely and the polymer layer stick to GF become thicker than GF in Figure $10 \mathrm{~b}$. It manifests much tighter connections were constructed due to 3-MPS modification. TES treated GF are dispersed randomly under the thin layer of the polymer matrix (as shown in Figure 6.9. e and f). The GFs are pulled out of the polymer matrix, and the surface cavities, which were created from the pulledout glass fibers, are smooth. This indicates the compatibility between GF and matrix is weak, coinciding with the tensile strength and the DMA results. Figure $10 \mathrm{~g}$ and h show that glass fibers are buried deeply under the membrane layer. There are some cross bridges on the interface between individual fiber and matrix, indicating the strong connection between them.

The coupling agent is like a bridge to connect GF and copolymer matrix. When the composite material is subjected to stress, the stress can be transferred from the membrane matrix to GF and dispersed over it. The GF fillers can also inhibit crack expansion.

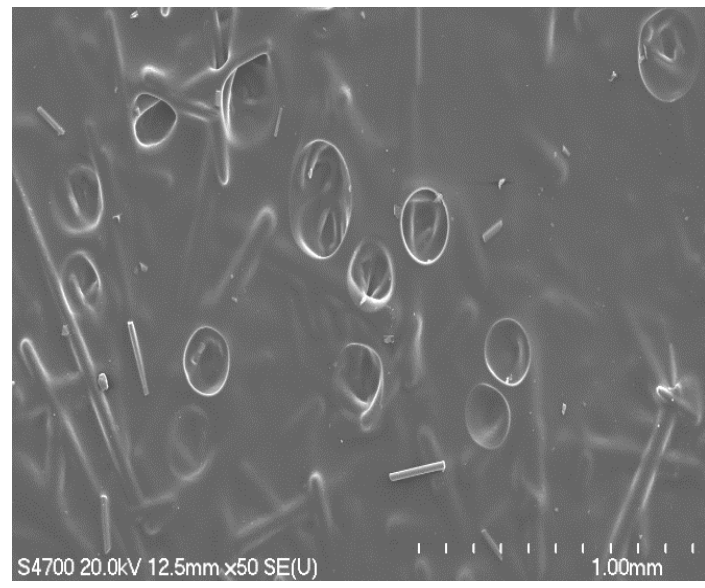

a)

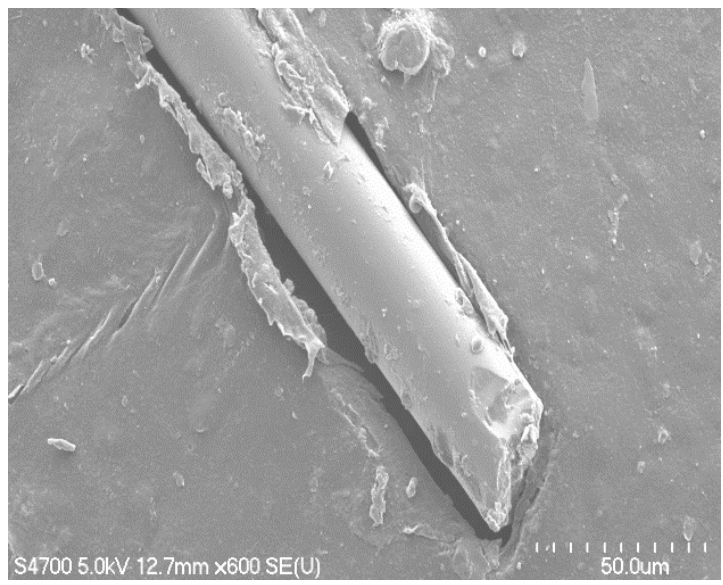

b)

Figure 6.8. a) b) CEMs composite with untreated glass fiber 


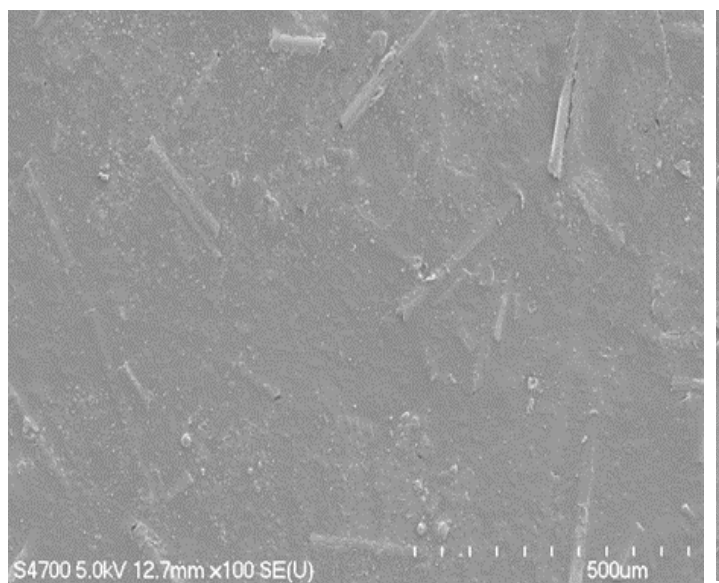

a)

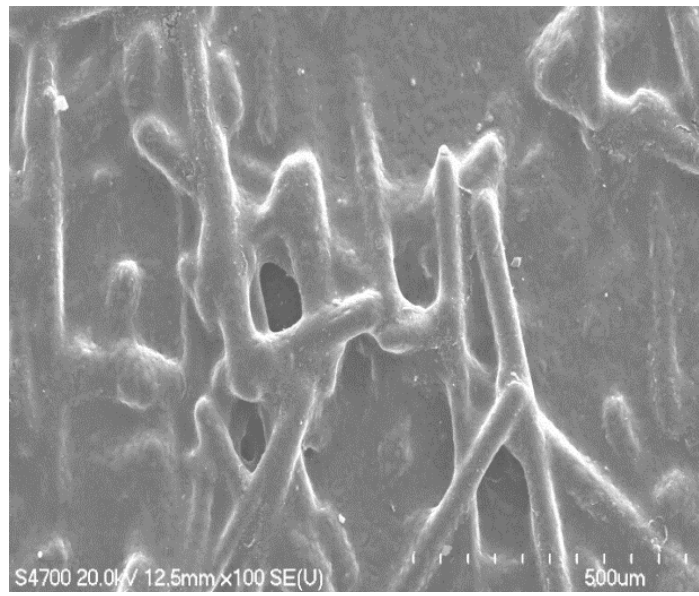

c)

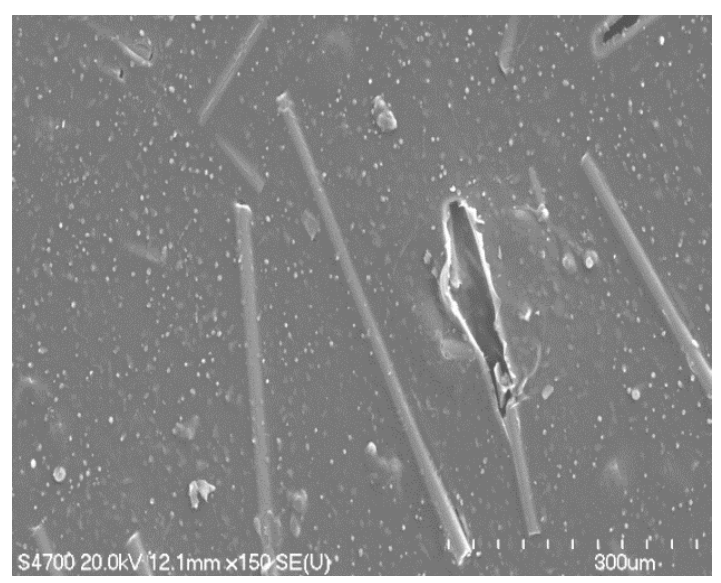

e)

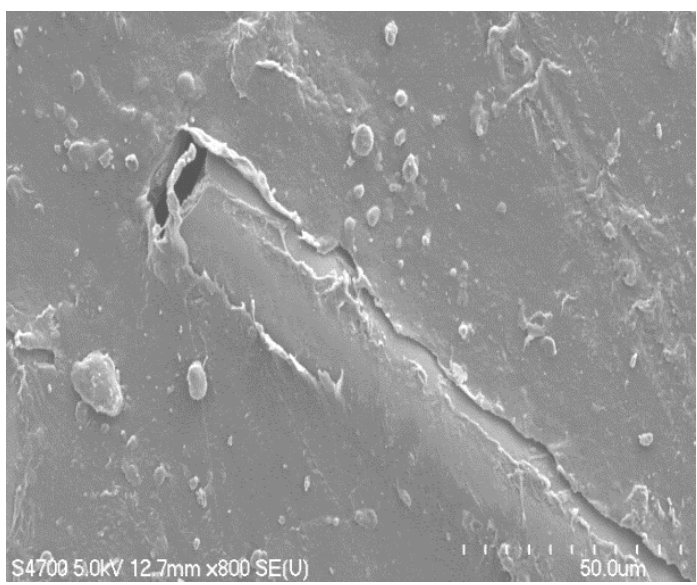

b)

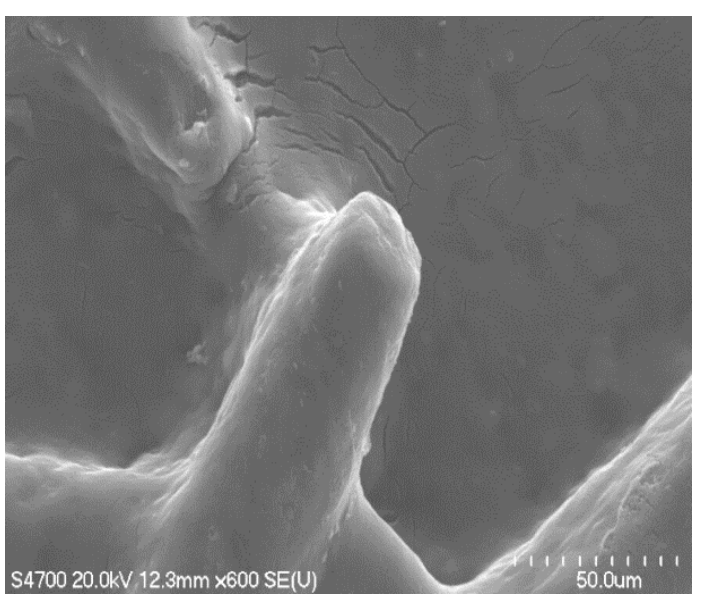

d)

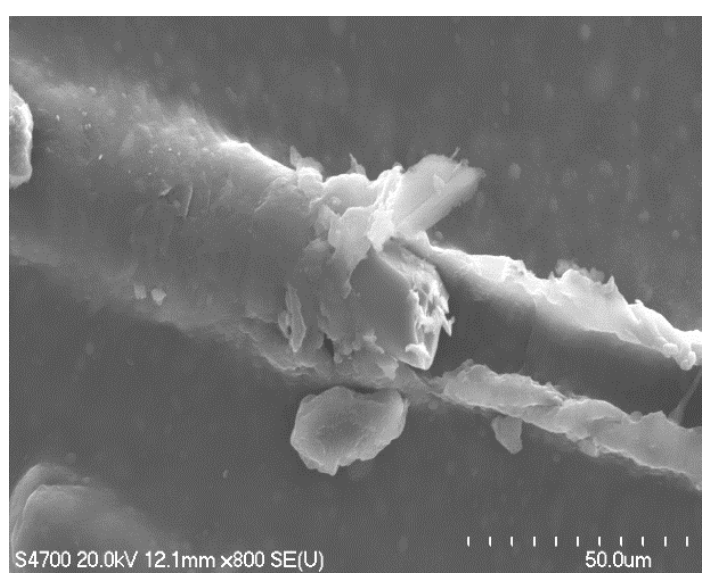

f) 


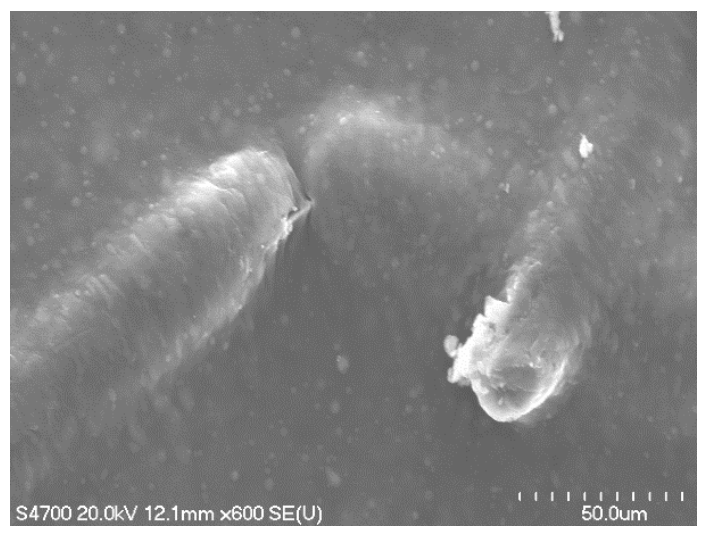

g)

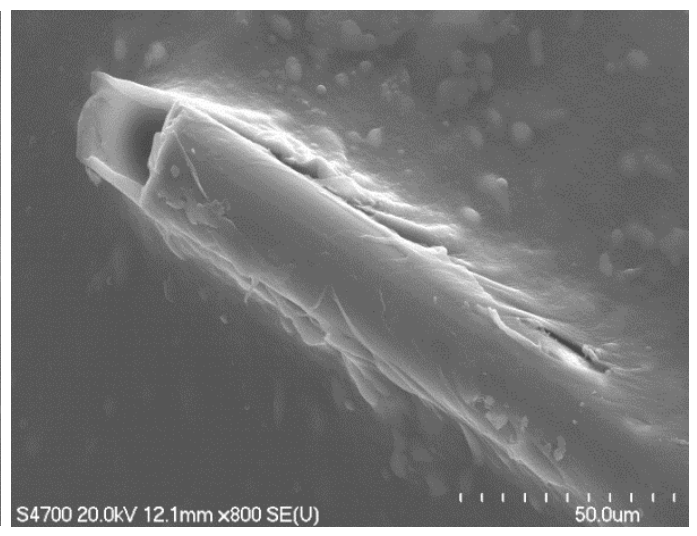

h)

Figure 6.9. CEMs composite with glass fiber modified by a) b) 1,6 bis; c) d) 3-MPS; e) f) TES; g) h) PP-g-MA

\subsubsection{FT-IR analysis}

The FT-IR spectrums of pure PE-g-PS copolymer and that with untreated GF and treated GF are shown in Figure 6.10. The right graph is the normalized FT-IR spectra of the left graph, showing the $\mathrm{C}-\mathrm{H}$ stretching region between 1340-1420 cm-1. Compare to the composite with untreated GF, the new peaks appearing around $1060 \mathrm{~cm}-1$ in 3, 4, 5 samples are due to asymmetric vibrations of ( $\mathrm{Si}-\mathrm{O}-\mathrm{Si}$ ), which are from the condensation between -OH in GF and the Si-OH groups in coupling agents. A new appearing peak at $1065 \mathrm{~cm}-1$ in the spectrum of sample 6 is attributed to asymmetric vibrations of C-O-Si bonds, which originated from the condensation between -OH in GF and C-OH in PP-gMA. Both emerging peaks are the evidence of forming chemical bonding between GF and coupling agents. The peaks intensities around $3660 \mathrm{~cm}-1$ in spectrum 3, 4, 5, and 6 amplified due to -Si-OH bonds from hydrolyzed 1,6 bis, 3-MPS, TES, and PP-g-MA. In addition, the peaks around $2850-3000 \mathrm{~cm}-1$ and $1400 \mathrm{~cm}-1$ are attributed to $\mathrm{C}-\mathrm{H}$ in methyl groups and $\mathrm{C}-\mathrm{H}$ in aliphatic, respectively. The intermolecular hydrogen bonding among the GF, coupling agents, and PE-g-PS matrix were characterized by these C-H stretching vibration. The $\mathrm{C}-\mathrm{H}$ stretching vibration regions of 3,4,5,6 spectrums presenting on the right graph are shift and degrade compared to the $\mathrm{C}-\mathrm{H}$ spectra in pure PS-g-PE. This change in the $\mathrm{C}-\mathrm{H}$ absorptions indicates the specific effect of hydrogen bonding between the matrix and coupling agents. Based on the above analysis, the possible reaction mechanism of GF, coupling agents and PE-g-PS copolymer are deduced as Scheme 6.1. 

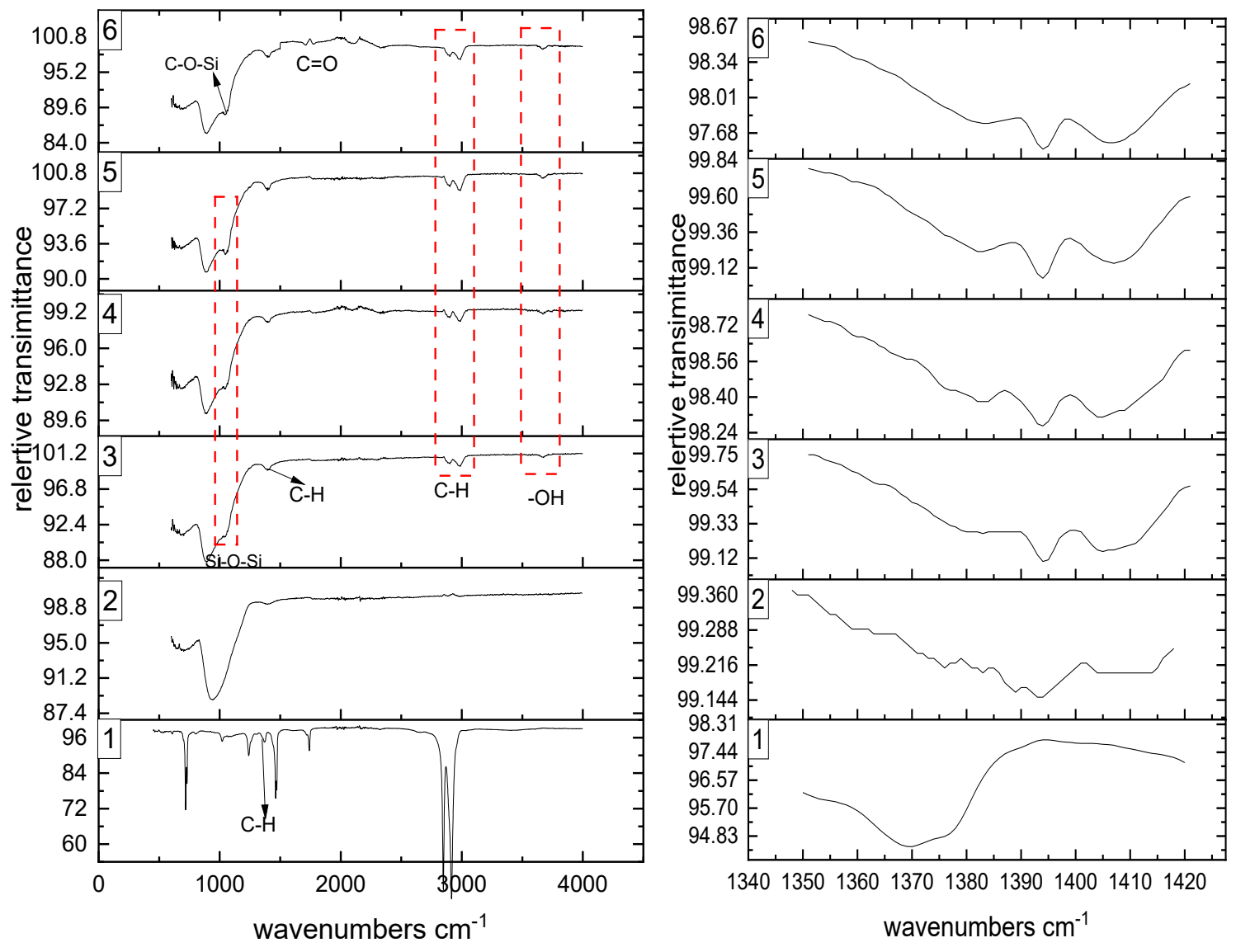

Figure 6.10. Left FT-IR spectrum (left) and normalized FTIR spectra in the C-H stretching region (right) of (1) pure PE-g-PS and PE-g-PS based composites with (2) untreated glass fiber (3) 1,6 bis; (4)3-MPS; (5) TES; (6) PP-g-MA 


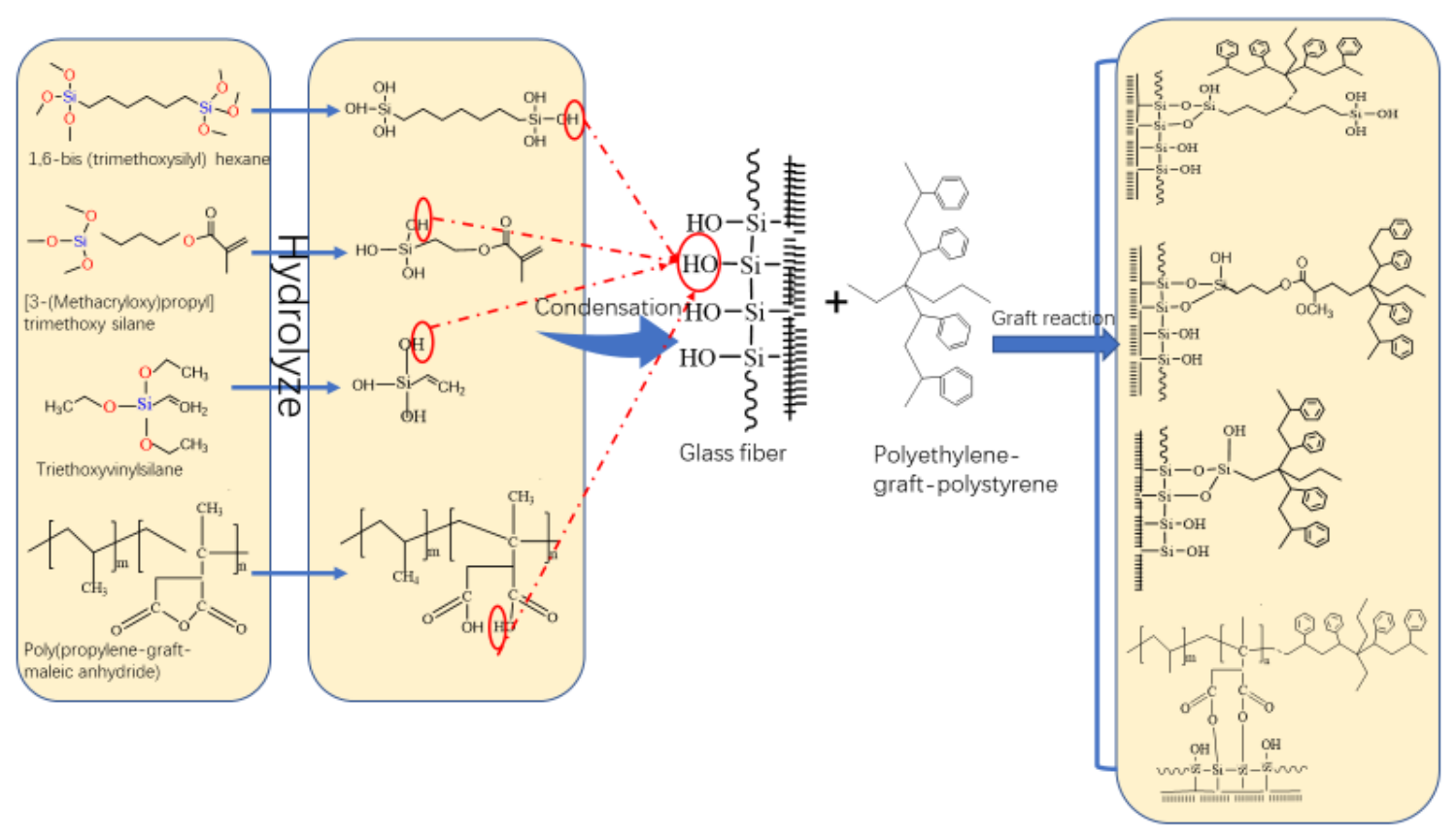

Scheme 6.1. Reaction mechanism of GF, coupling agents and PE-g-PS copolymer.Effect of CEMs composite with modified GF on ion exchange capacity

Since the mechanical properties are strongly related to the coupling agent's concentration, the effects of concentration on formation of chemical bonding were investigated by the FT-IR spectrum. Figure 6.11 presents different samples' FT-IR spectrums with GF treated by increasing 3-MPS concentration. The characteristic peaks appearing at $1002 \mathrm{~cm}-1$ (Si-O-Si), $1130 \mathrm{~cm}-1$ (Si-O-Si), and $805 \mathrm{~cm}-1$ (Si-O3CH2-) became sharpest when 3-MPS concentration reached 2\%. Increasing 3-MPS concentration weakened corresponding peak intensities. The less sharp characteristic peaks indicate fewer amounts of Si-O-Si bonds generated between GF and coupling agents. The $-\mathrm{C}-\mathrm{H}$ peaks attributed to methyl groups and aliphatic have the same trends as Si-O-Si peaks, indicating the more PE-g-PS can be attached on GF when GF was treated by $2 \% 3$-MPS hydrolyzed solution. These results demonstrate that the $2 \% 3$-MPS concentration has a superior modified effect on GF. 


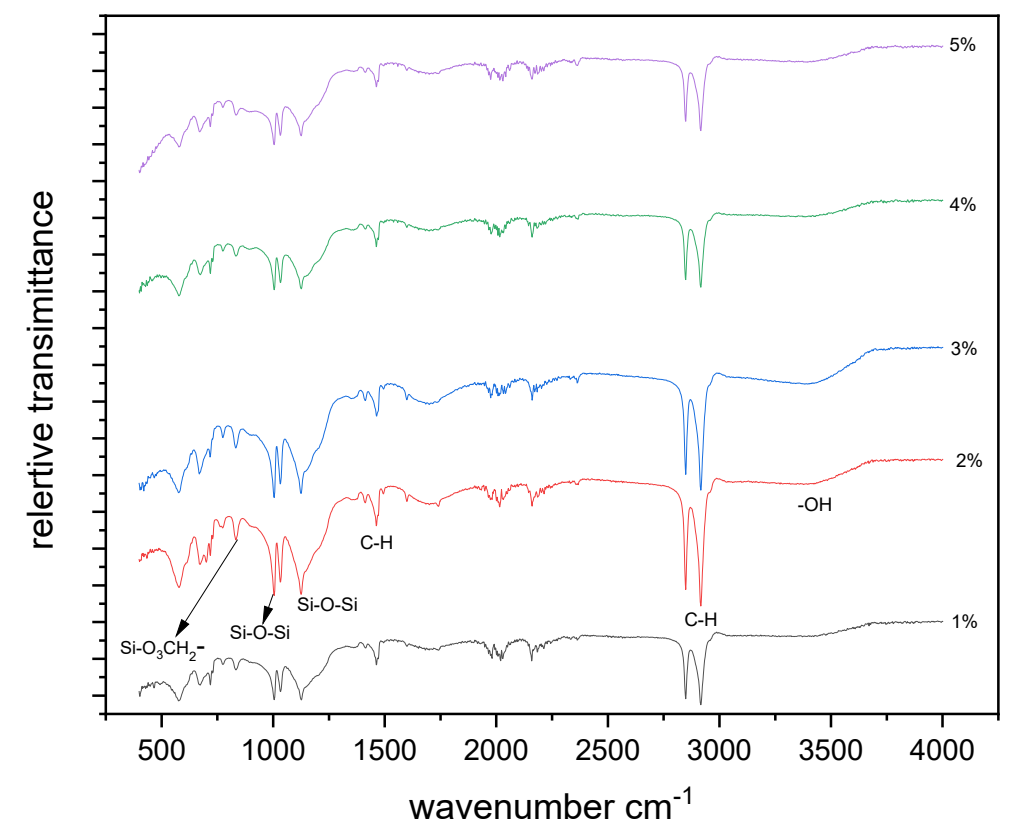

Figure 6.11. FT-IR spectrum of PE-g-PS based composite with GF treated by different 3MPS concentration

\subsubsection{Effect of GF on Ion Exchange Capacity}

The concern of adding fillers is that it may hinder ions transport and decrease ion exchange capacity. The result in Figure 6.11. shows the CEM without GF has the largest IEC value, reached $1.78 \mathrm{mmol}$ g- 1 . The IEC of CEM composites with treated GF decreases slightly, 2-6\% lower than CEMs without GF. The negative influence of adding GF on IEC value is in an acceptable range. However, the addition of untreated GF decreased IEC value tremendously due to the vast emerging holes caused by GF insertion intercepted the ion transportation channels. The coupling agents could fill those vacancies, making the GF combined with the matrix tightly. When cations transport through hydrated membranes, they obey the "diffusion mechanism". Hydrated cation $(\mathrm{H} 3 \mathrm{O}+)$ diffuses through the aqueous medium in response to the electrochemical difference in this mechanism. The water connected cation $\left(\mathrm{H}+\left(\mathrm{H}_{2} \mathrm{O}\right) \mathrm{x}\right)$ carries one or more water molecules transported through the membrane. Under this condition, the cations could bypass the obstacles and conduct through branch ionic groups of PE-g-PS membrane when encountering GF. 


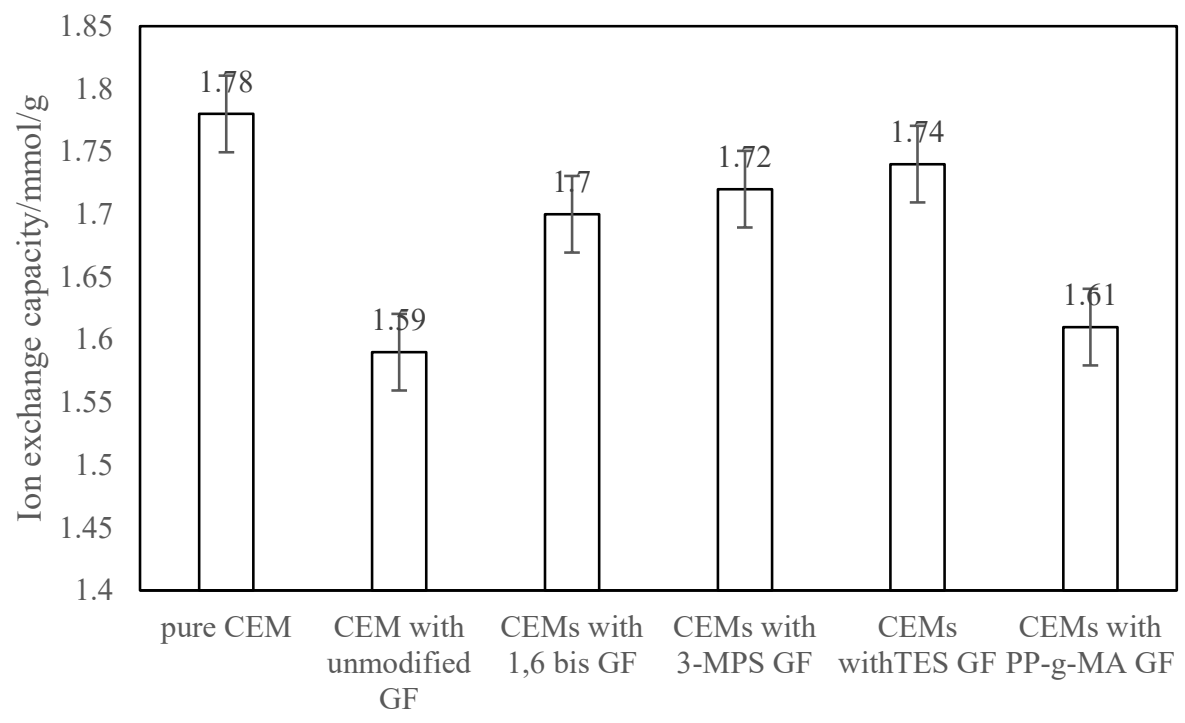

Figure 6.11. Effect of GF with different coupling agent on IEC of CEMs

\subsubsection{Effect of GF/CEMs composite on water uptake and swelling rate}

Adding GF into CEMs also affects water uptake and swelling rate due to the hydrophilic nature of GF. Figure 6.12. shows that the water uptake ability enhanced moderately after adding modified GF. The GF/CEM composite with 1,6 bis treated GF has the highest water uptake. Accordingly, the swelling rates of composite with modified GF also increased due to a mass of water attracted by the GF. On the other hand, as fillers, the GF could hinder composites' deformation to some extent. Only if the poor connection existed between GF and matrix would result in the deformation force aroused by swelling is much larger than GF's resistance. The blank sample is this case in which the swelling rate is relatively high due to the absence of chemical bonding between the GF and composite matrix. It also indicates that all the samples' water uptake values and swelling rate tend to be stable after immersing in $0.5 \mathrm{~mol}$. L-1 NaCl for $2 \mathrm{~h}$. Water uptake is a vital property since it positively correlates to IEC. However, excessive water uptake would result in swollen membrane. Thus, the enhanced water uptakes of CEMs with 3-MPS, PP-g-MA, and TES treated GF lead to a higher IEC but increase the risk of unstable dimension. 


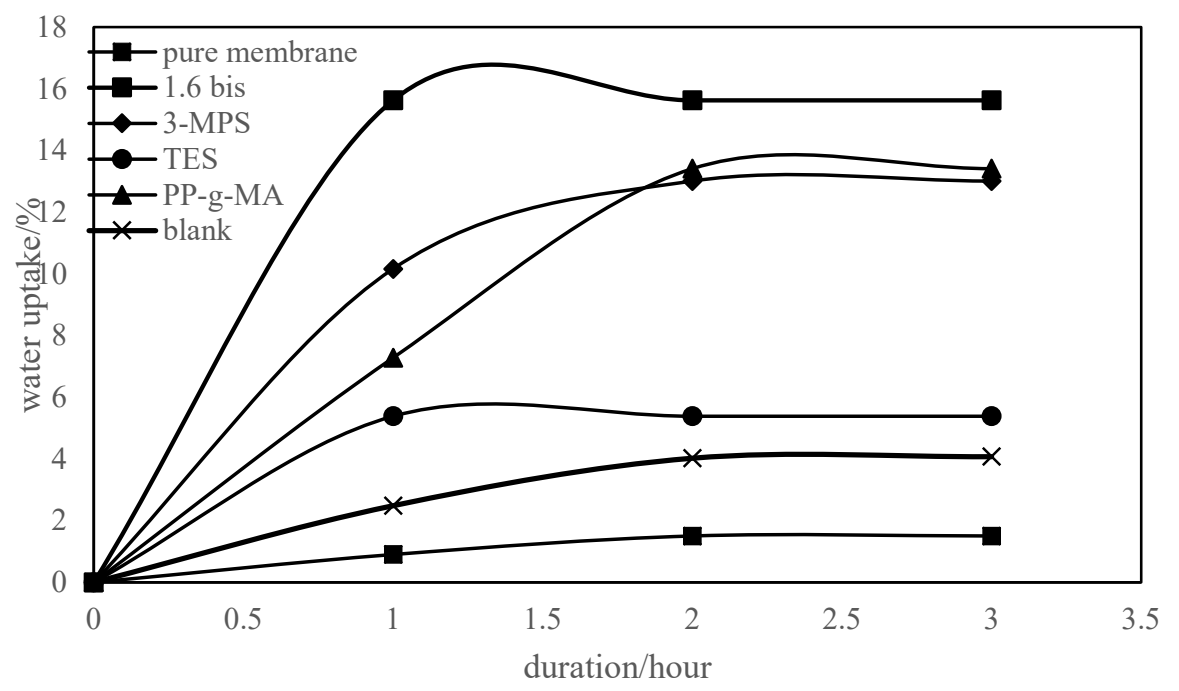

Figure 6.12. Effect of coupling agent on water uptake of CEMs with different GF

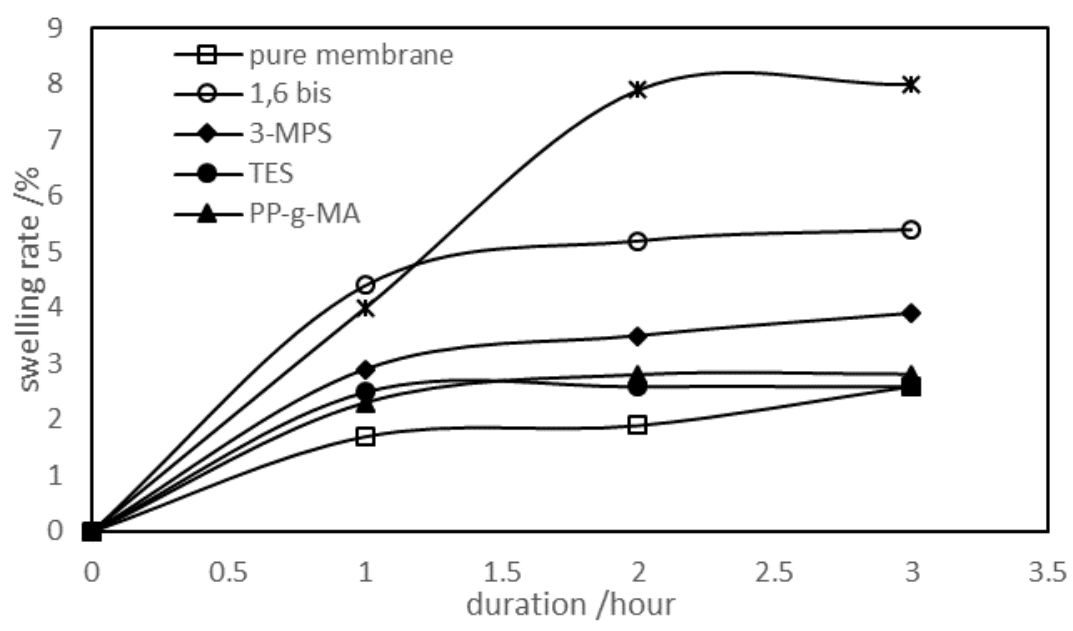

Figure 6.13. Swelling rate of composites with different modified GF as functions of duration

\subsection{Summary}

This research provided information about interfacial adhesion and mechanical properties improvement effects of four coupling agents on GF/CEM composite. The addition of modified GF enhanced the tensile strength and tensile modulus of GF/CEM composites but cannot make composites suffer too much elongation. The interfacial adhesion between matrix and GF, reflected by tan delta and adhesive effect parameter, were improved with the addition of coupling agents due to the formations of Si-O-Si bonds 
between GF/coupling agent, and hydrogen bonding among the GF, coupling agents and matrix . The 3-MPS treated GF showed satisfactory modification consequence, brings the most robust interface adhesion to composite, and leaded to the highest tensile strength. However, with increasing 3-MPS's concentration to treat GF, the mechanical properties and interfacial adhesion got worse. The unmodified GF negatively influenced composite's mechanical properties due to the destruction of the membrane's integrity and formation of micro-holes around the GF. The water uptake values increased after adding modified GF into CEMs. However, the IEC of GF/CEM composite decreased slightly. The swelling rate for unmodified GF/CEM composite is relatively high due to the absence of chemical bonding between the GF and composite matrix.

This research provided a way of strengthening GF/CEM composite and pointed out which functional groups included in coupling agents could be useful to GF reinforced composite. The future studies of GF reinforced ion exchange membrane could use these conclusions as references. Future studies might consider other surface modifiers with similar characteristic structures or functional groups as effective coupling agents for GF reinforced CEM composite. 


\section{Dually acid-doped functionalized graphene oxide/sulfonated poly (ethylene-co-styrene) cation exchange membrane composite}

\subsection{Introduction}

Today, separation membrane techniques have been widely applied in reverse osmosis, nanofiltration, ultrafiltration, microfiltration, pervaporation separation and electrodialysis process $[1,126]$. Electrodialysis is one of the separation techniques in which the counterions are selected spontaneously and permeate through membranes under the drag of electrical potential difference. Ion exchange membranes (IEM), as one of the core components of electrodialysis, have been widely applied to seawater desalination as well as brackish water concentration, pharmaceutical purification, water softening processes and fuel cell separators etc. [4]. To meet processing requirements of plating industry for heavy metals concentration, ion exchange membranes are expected to have high ionic conductivity, selectivity, excellent thermal and mechanical stability. The most used polymer electrolyte membrane is Nafion, which is considered to have high proton conductivity and chemical stability but suffer from low ionic conductive performance at elevate temperature. Therefore, the polymer electrolyte membrane with excellent electrochemical properties as well as mechanical and chemical stability at elevate temperature is desired.

Generally, ionic conductivity of IEM is determined by many factors, such as water content in membrane matrix, external electrolyte concentration, hydration conditions, spatial distribution of fixed ions at membrane surface [124,127]. Several tries of improving ions conductivity in IEM have been explored. Enhancing water retention for IEMs is a promising way to achieve high conductivity [127,122]. IEM contains two types of water, bound water and bulk water [124]. The bound water, associating with ion hopping mechanism, drive cations hop from one functional ionic site $\left(\mathrm{SO}_{3} . \mathrm{H}_{3} \mathrm{O}^{+}\right)$to another until cross the membrane. In this mechanism, bound water as ions carriers, provided sufficient cations transporting channels in process. The bulk water, connecting with cations $\left(\mathrm{H}^{+}\left(\mathrm{H}_{2} \mathrm{O}\right) \mathrm{x}\right)$, transfers through membrane with the cations as the result of electroosmotic mechanism. The incorporation of hydrophilic fillers, such as silica and zeolites, was proved to improve the bulk water content of IEM [119,122]. However, at high temperature situation, the incorporation of uncharged fillers results in rapid water release due to weak connection with water molecules, making low ions conductivity for IEM [122, 126,127]. In addition, the phase separated structure composed by hydrophilic groups and hydrophobic matrix, has also been reported to improve ions conductivity [128]. The interconnected hydrophilic groups produce ions conductivity channels for ions transport. Moreover, grafting sulfonic function group on matrix chains was verified as an effective way to enhance ions conductivity for cation exchange membrane $[121,127]$. 
Although the high sulfonic acid sites could lead high conductivity for cation exchange membrane, the sulfonic acid sites often result in the highly soluble in aqueous solution and excessive swollen in membrane due to the highly hydrophilic property [120].

Recent research has focused on incorporation of organic or inorganic fillers with charged groups into the ion exchange membranes, which intended to address the issues of rapid water content release and mechanical durability at elevated temperature [121-123]. Graphene oxide (GO), containing a lamellar graphene framework with hydroxyl (-OH), carboxyl $(-\mathrm{COOH})$ and carbonyl $(\mathrm{C}=\mathrm{O})$ groups, was considered as attractive organic fillers $[120,124]$. Because of the presence of these oxygen groups, GO can be well hydrated. Furthermore, when GO incorporates acidic functional groups, the intermolecular distance of GO nanosheet will furtherly expand due to the electrostatic repulsion $[131,132]$. This expanded distance can provide abundant ions conducting paths, thus enhance water retention and ions conductivity. Recently, the sulfonated reduced graphene oxide nanosheets with negatively charged sulfonic acid groups, was demonstrated to enhance the monovalent anions conductivity of anion exchange membrane [122]. GO sulfonated derivatives has been widely reported as fillers to enhance water retention and mechanical stability of IEM $[116,128]$. The multifunctional composite proton exchange membrane with sulphonic acid $\left(-\mathrm{SO}_{3} \mathrm{H}\right)$ membrane matrix and phosphonic acid propyl silane graphene oxide fillers have attracted broad attentions $[117,121]$. It has been demonstrated that the addition of $-\mathrm{PO}_{3} \mathrm{H}_{2}$ based graphene oxide in sulphonic acid matrix could enhance water retention, proton conductivity and mechanical stability due to the multi-functional groups provide a hydrophobic-hydrophilic phase separation for proton conducting channels $[123,126]$.

Herein, for the first time, we present a chemical strategy of synthesizing multi functionalized GO with both phosphonic and sulfonic acid functional groups, then incorporating with PE-g-PS based CEM to enhance ionic conductivity and mechanical properties. The effect of different concentration of dual functionalized GOs on membrane properties were also investigated.

\subsection{Products design and synthesis procedure}

The basic idea of synthesizing graphene oxide derivate is modifying $\mathrm{GO}$ to create active sites to accept ionic groups. Then graft ionic groups such as- $\mathrm{PO}_{3} \mathrm{H}_{2}$ and $-\mathrm{SO}_{3} \mathrm{H}$ on the modified GO. These ionic groups also connect with functional groups of membrane through hydrogen bonds or electrostatic interaction. The synthesizing procedure of GO, modified graphene oxide (MGO), acid functionalized graphene oxide (graphene oxide derivation) is shown in scheme 7.1. Graphene oxide was prepared from graphite powder by modified Hummers method [120] as described in 3.2.4.1. Graphene oxide converted into graphene oxide derivate through (3-aminopropyl) trimethoxy silane modification and sonication [122]. This modification provides graphene oxide an interface to be grafted with ionic groups $-\mathrm{PO}_{3} \mathrm{H}_{2}$, which was carried out by using phosphorous acid at the 
presence of formaldehyde. Then, another acid group--- $\mathrm{SO}_{3} \mathrm{H}$ was connected by using sulfanilic acid with ultrasonic process.
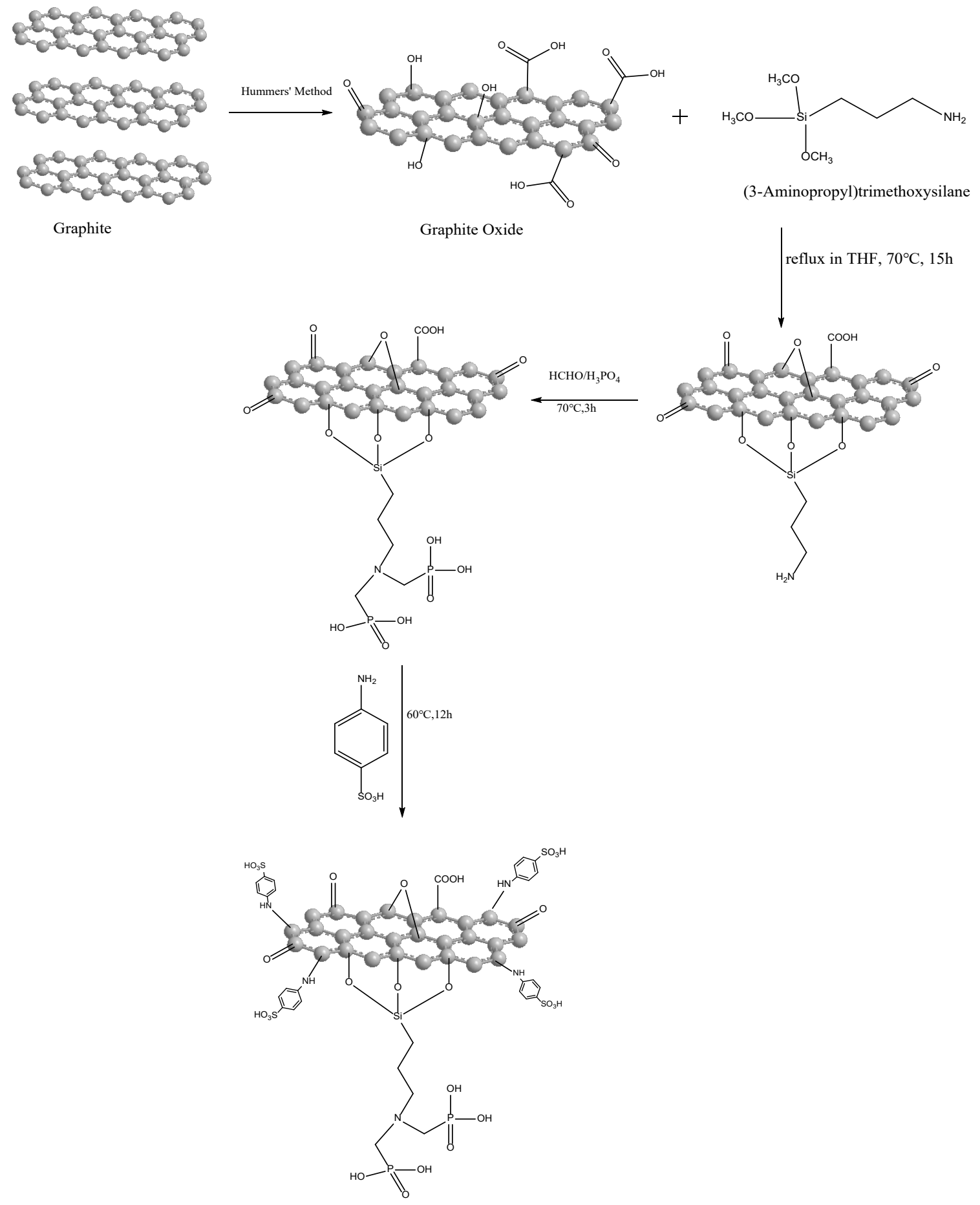
Scheme7.1. Scheme route for the preparation of graphene oxide, modified graphene and graphene oxide derivation

\subsection{Experimental results and discussion}

\subsubsection{Characterization of the functionalized graphene oxide and membrane composites}

\subsubsection{FT-IR analysis}

The FTIR spectrums of graphite, GO, MGO, phosphonic acid graphene oxide(P-MGO), sulfonated graphene oxide(S-MGO), PS-MGO are illustrated in Figure 7.1. Compare to the raw graphite, the IR spectrums of the synthesized materials show a broad band around $3200-3600 \mathrm{~cm}^{-1}$, which is due to the existence of large number of hydroxyl groups of GO bending vibration. Besides, the spectrums of S-MGO and PS-MGO show lower spectra broadness and higher intensity, which attribute to the generation of C-N (amide group) between GO and sulfanilic acid molecular. An absorption band at $1452 \mathrm{~cm}^{-1}$ on GO correspond to $\mathrm{O}-\mathrm{H}$ stretching of $-\mathrm{COOH}$ group. This absorption band has less intensity on MGO, P-MGO, S-MGO and PS-MGO due to the replacement of O-Si-C originated from (3-aminopropyl) trimethoxy silane condensation. The IR spectrums of GO, MGO and GO derivates exhibit the absorption peaks at $1740 \mathrm{~cm}^{-1}$ attribute to the $\mathrm{C}=\mathrm{O}$ stretching vibrations, suggesting the presence of hydroxyl. The absorption peaks at 1110 and 1630 $\mathrm{cm}^{-1}$ on GO attributable to the C-O-C stretching and C-O stretching vibration, respectively. Furthermore, the presence of free terminal primary amine groups in MGO was confirmed by the absorption bands at $\sim 1627 \mathrm{~cm}^{-1}$ and $79 \mathrm{~cm}^{-1}$ due to the $-\mathrm{N}-\mathrm{H}$ bend and $-\mathrm{N}-\mathrm{H}$ wag of aliphatic primary amine respectively. The peaks at 1051 and $744 \mathrm{~cm}^{-1}$ for synthesized material are the characteristic peaks of Si-O-C and Si-C, demonstrating the $\mathrm{GO}$ has been modified by (3-aminopropyl) trimethoxy silane successfully. Moreover, absorption peaks at $\sim 1335 \mathrm{~cm}^{-1}$ on P-MGO and PS-MGO confirmed the presence of $\mathrm{PO}_{3} \mathrm{H}$ groups. The peaks at $1115 \mathrm{~cm}^{-1}$ for S-MGO and PS-MGO demonstrates the presence of $-\mathrm{SO}_{3} \mathrm{H}$ functional groups. When sulfanilic acid was grafted onto the MGO surface, the spectra of S-MGO and PS-MGO showed band at about $1030 \mathrm{~cm}^{-1}$, which was assigned to the symmetric stretching vibration of $\mathrm{S}=\mathrm{O}$. 


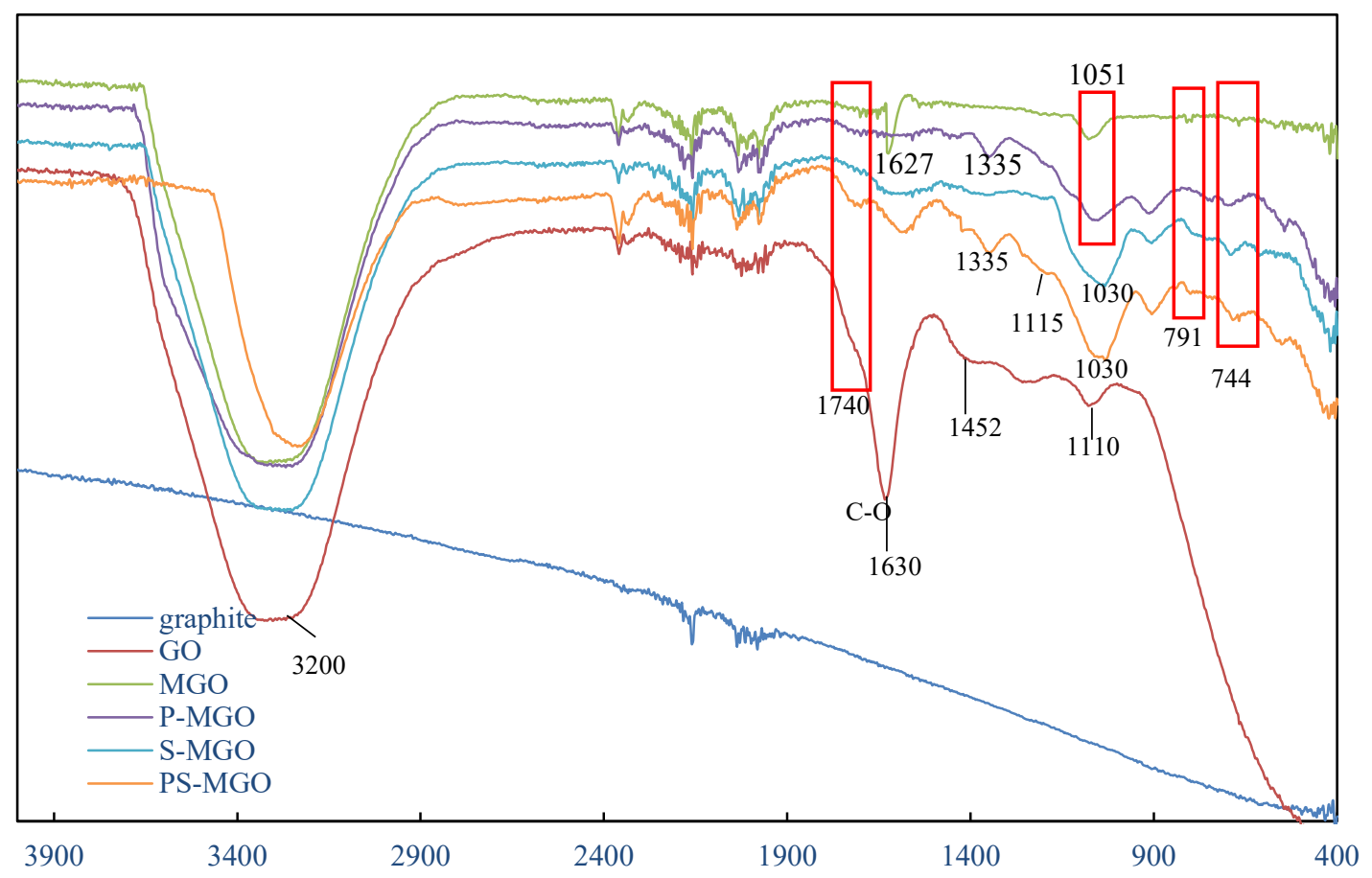

Figure 7.1. FTIR of raw graphite, GO, modified GO and functionalized GO

\subsubsection{XRD analysis}

To confirm raw graphite, GO and GO derivate structure and their interlayer distance, the XRD patterns are illustrated in figure 7.2. The characteristic peak of graphite located at 2 $\theta=26.5$, corresponding the graphite interlayer distance $\mathrm{d}=0.34 \mathrm{~nm}$. The diffraction peak of GO showed a shift to $2 \theta=14.16$ with a interlayer distance $\mathrm{d}=0.63 \mathrm{~nm}$, which demonstrated the layer distance of $\mathrm{GO}$ was expanded due to the appearance of carboxylic acid, hydroxyl and epoxy ether functional groups between GO layers [115]. The broadened diffraction peak of MGO at $2 \theta=20.8$ with the decreasing interlayer distance $(\mathrm{d}=0.43 \mathrm{~nm})$ indicates the $\mathrm{GO}$ was successful modified by (3-aminopropyl) trimethoxy silane. This is due to the oxygen groups contained in $\mathrm{GO}$ were partly removed and the formation of $\pi-\pi$ results in interlayer spacing reduction. Another obvious peak around $2 \theta=6.18$ suggests the appearing of small fraction of multilayers. The three types of graphene oxide derivations appear broadening and intensity decline peaks, shifting to higher $2 \theta$ compared to the GO crystalline peak. This was attributed to the disorder of phase-separated structure and incorporation of amorphous ionic clusters, such as $\mathrm{SO}_{3} \cdot \mathrm{H}^{+}$or $\mathrm{PO}_{3} \cdot 2 \mathrm{H}^{+}$. 


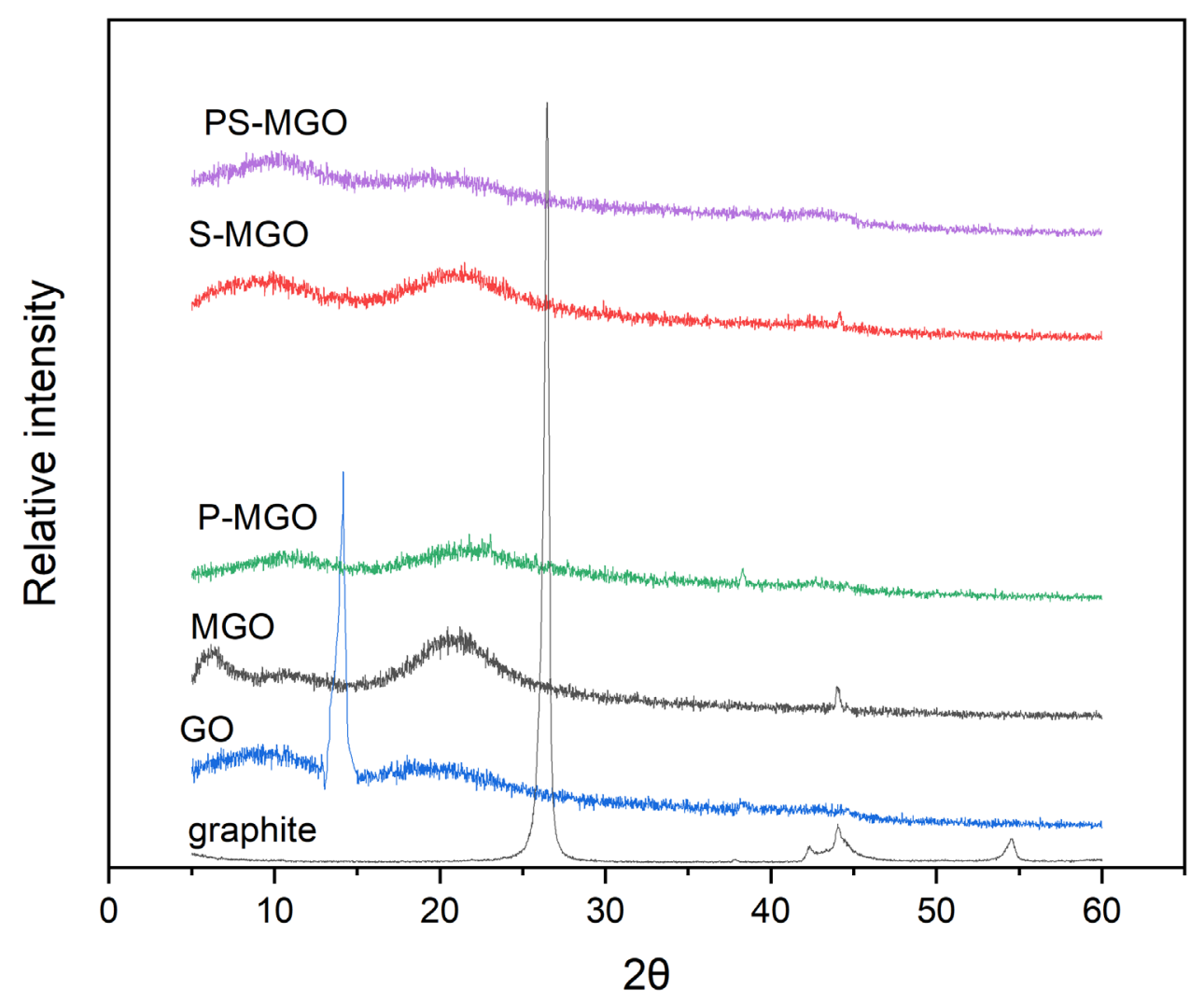

Figure 7.2. XRD pattern of graphite, GO, MGO, P-MGO, S-MGO, PS-MGO

\subsubsection{XPS characterization and analysis}

The chemical composite and structure of modified and functionalized GO were investigated by XPS in this section. The Figure 7.3a) displayed comparation of wide range XPS patterns of GO and MGO, three new peaks appearing in MGO at binding energy of 400.6, 153.4 and 102.2, corresponding the N 1s, Si 2s and Si 2p1/2, respectively. These emerging peaks confirm the GO has been modified by (3aminopropyl) trimethoxy silane successfully. Compare to the MGO, the spectra of SMGO and PS-MGO presented in Figure 7.3 b) and c) both appeared new peaks. However, the intensity of peaks Si 2 s and Si 2 p $1 / 2$ weakened. The peak located at $169.4 \mathrm{eV}$ in S-MGO spectra attributed to $\mathrm{S} 2 \mathrm{~s}$, indicating the successful attachment of sulfonic acid onto the MGO surface. Another weak peak appearing at $134.2 \mathrm{eV}$ attributed to $\mathrm{P} 2 \mathrm{p}$, verified the functionalized GO containing P. The decreased intensities of two $\mathrm{Si}$ peaks are attributed to the addition of sulfonic acid and the replacement of $\mathrm{H}^{+}$of $-\mathrm{NH}_{2}$ by $\mathrm{PO}_{3} \mathrm{H}_{2}$. The atomic percentages of GO, MGO, S-MGO and PS-MGO are listed in Table 7.1. The MGO has shown a great Si enhancement $(9.07 \%$, respectively) compare to the pristine GO due to the modified effect of (3-aminopropyl) trimethoxy silane. The $\mathrm{N}$ content has elevated moderately due to the addition of $-\mathrm{NH}_{2}$ contained in (3- 
aminopropyl) trimethoxy silane. However, the $\mathrm{N}$ content continually decrease after functionalization due to appearance of S and $\mathrm{P}$ from sulfanilic acid and phosphoric acid, respectively.
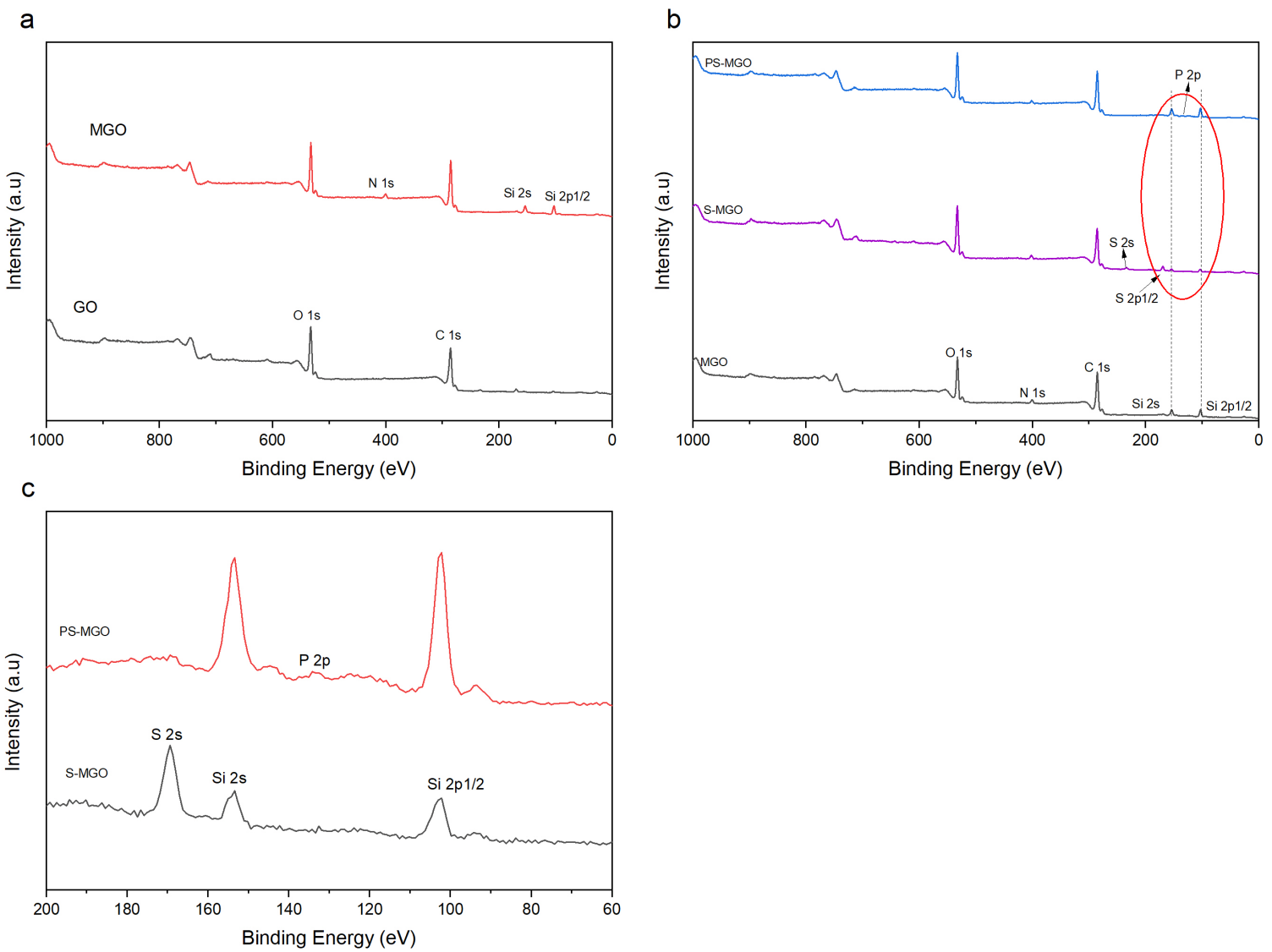

Figure 7.3 Wide range of XPS spectra of a) GO and MGO b) MGO S-MGO and PS$\mathrm{MGO}$ and $\mathrm{c}$ ) S-MGO and PS-MGO ( $\mathrm{c}$ is the high magnification of spectra in red circle of b)

Table 7.1 Atomic percentages of pristine GO, modified MGO and functionalized S-MGO and PS-MGO determined from XPS survey scan

\begin{tabular}{lllllll}
$\begin{array}{l}\text { Sample } \\
\text { name }\end{array}$ & $\mathbf{O}$ & $\mathbf{C}$ & $\mathbf{N}$ & $\mathbf{S i}$ & $\mathbf{S}$ & $\mathbf{P}$ \\
\hline GO & 23.89 & 75.62 & 0.49 & - & - & - \\
\hline MGO & 21.64 & 65.84 & 3.45 & 9.07 & - & - \\
\hline S-MGO & 25.7 & 62.97 & 3.09 & 4.52 & 3.72 & -
\end{tabular}


To investigate the chemical structure of new appearing peak furtherly, deconvulated XPS spectrums of $\mathrm{C} 1 \mathrm{~s}, \mathrm{O} 1 \mathrm{~s}$ and $\mathrm{N} 1 \mathrm{~s}$ of GO, MGO, S-MGO and PS-MGO are depicted in figure 7.4, 7.5 and 7.6. The $\mathrm{C} 1 \mathrm{~s}$ XPS peaks of $\mathrm{GO}$ are composed of three components including $\mathrm{C}-\mathrm{C}, \mathrm{C}-\mathrm{O}-\mathrm{C}$ and $\mathrm{O}-\mathrm{C}=\mathrm{O}$ with the binding energy at $284.62 \mathrm{eV}, 286.22 \mathrm{eV}$ and 288.42 respectively. These peaks shifted to $284.87 \mathrm{eV}, 286.57 \mathrm{eV}$ and $288.77 \mathrm{eV}$ in SMGO with new peak at $283.47 \mathrm{eV}$ representing C-Si. Furthermore, the C-C peak of PSMGO became more intense and shifted to higher binding energy due to the phosphoric acid addition. Comparison of O 1s signals in GO, S-MGO and PS-MGO clearly showing new peaks appearing in S-MGO and PS-MGO after functionalization, assigned to the Si$\mathrm{O}$ bond. Combination of new appearing of $\mathrm{C}-\mathrm{Si}$ bonds in figure $7.3 \mathrm{~b}$ ) and c), the new Si$\mathrm{O}$ peak showing in deconvulated XPS confirmed the formation of O-Si-C after GO modification. The $\mathrm{C}=\mathrm{O}$ and $\mathrm{C}-\mathrm{OH}$ peaks signal have slightly changed in $\mathrm{O} 1 \mathrm{~s}$ XPS spectra of S-MGO and PS-MGO. Moreover, the peak intensity observed for S-MGO are stronger than that for PS-MGO due to the addition of phosphoric acid with large molecule weight. The wide range XPS spectrums of MGO, S-MGO and PS-MGO have demonstrated the existence of nitrogen but can't distinguish the atomic states among them. Through the high-resolution spectra curves, the $\mathrm{N}$ 1s spectra of MGO (figure 7.6 a)) is assigned to $\mathrm{C}-\mathrm{N}$ and $\mathrm{C}-\mathrm{NH}_{2}$. The $\mathrm{N} 1 \mathrm{~s}$ spectra of S-MGO exhibited a new binding signal $\left(\left(\mathrm{C}_{6} \mathrm{H}_{4}\right) \mathrm{NH}\right)_{\mathrm{x}}$, attributed to the addition of sulfonic acid. While the $\mathrm{N}$ 1s spectra of PS-MGO is compromised by $-\mathrm{C} \equiv \mathrm{N}$ and $\mathrm{N} \equiv \mathrm{C}-\mathrm{CH}_{2}$ which demonstrated the $\mathrm{C}-\mathrm{NH}_{2}$ group has been replaced by $-\mathrm{C}-\mathrm{N}-\mathrm{CH}_{2}-\mathrm{H}_{2} \mathrm{PO}_{3}$. 

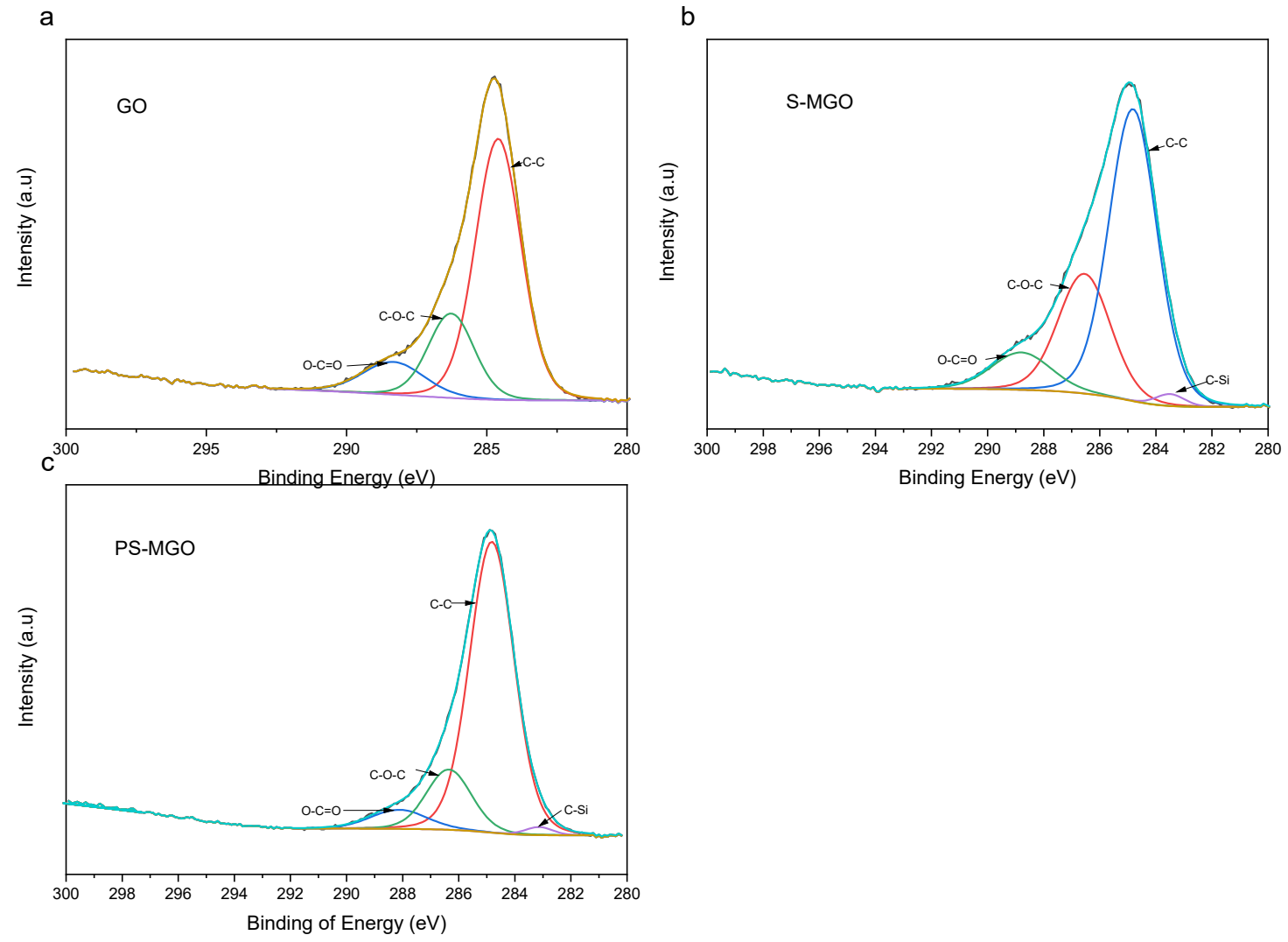

Figure 7.4 Deconvoluted XPS spectra in the C1s region for (a) GO (b) S-MGO and (c) PS-MGO

This is consistent with $\mathrm{O}$ 1s result. 

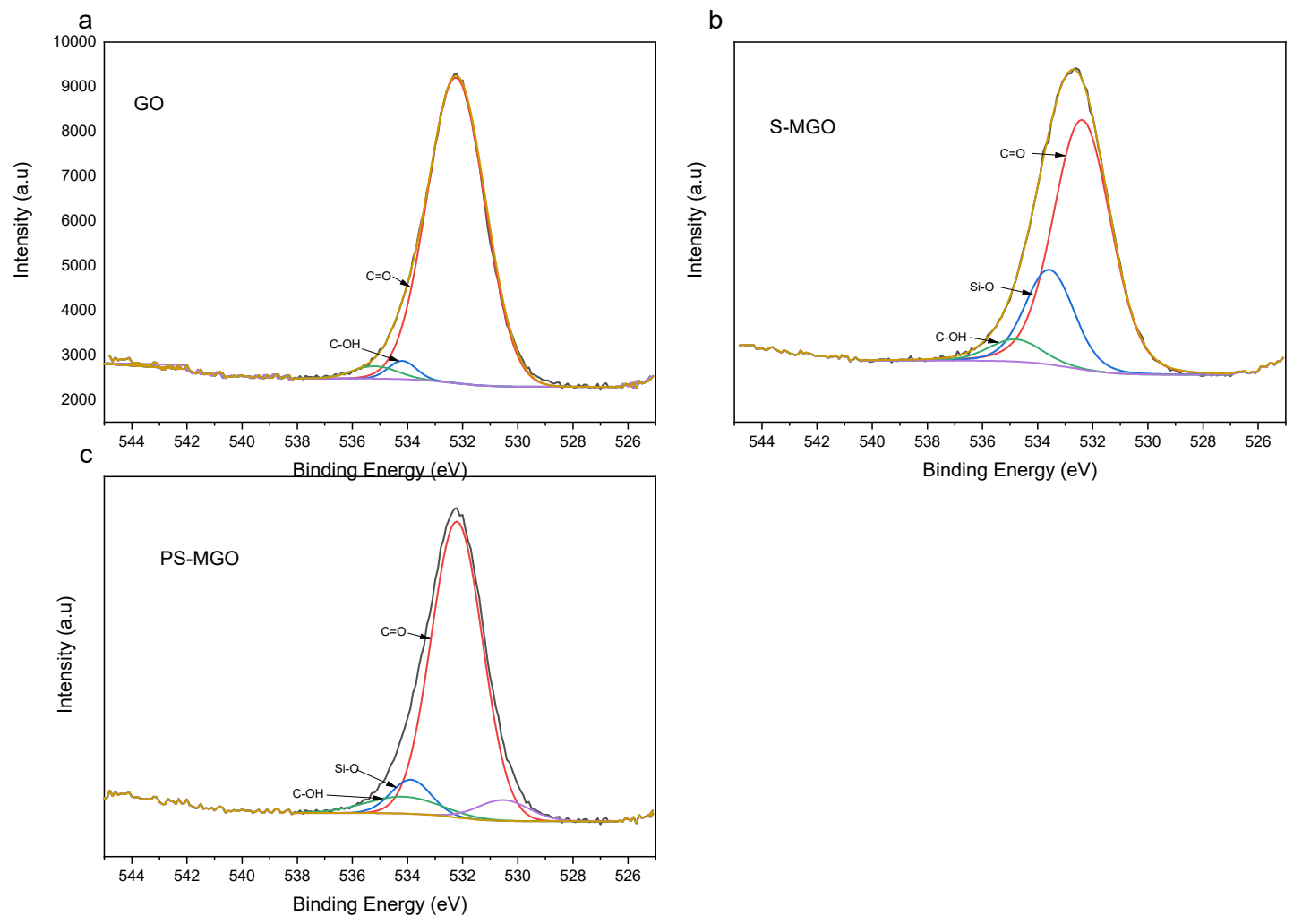

Figure 7.5. Deconvoluted XPS spectra in the O1s region for (a) GO (b) S-MGO and (c) PS-MGO 

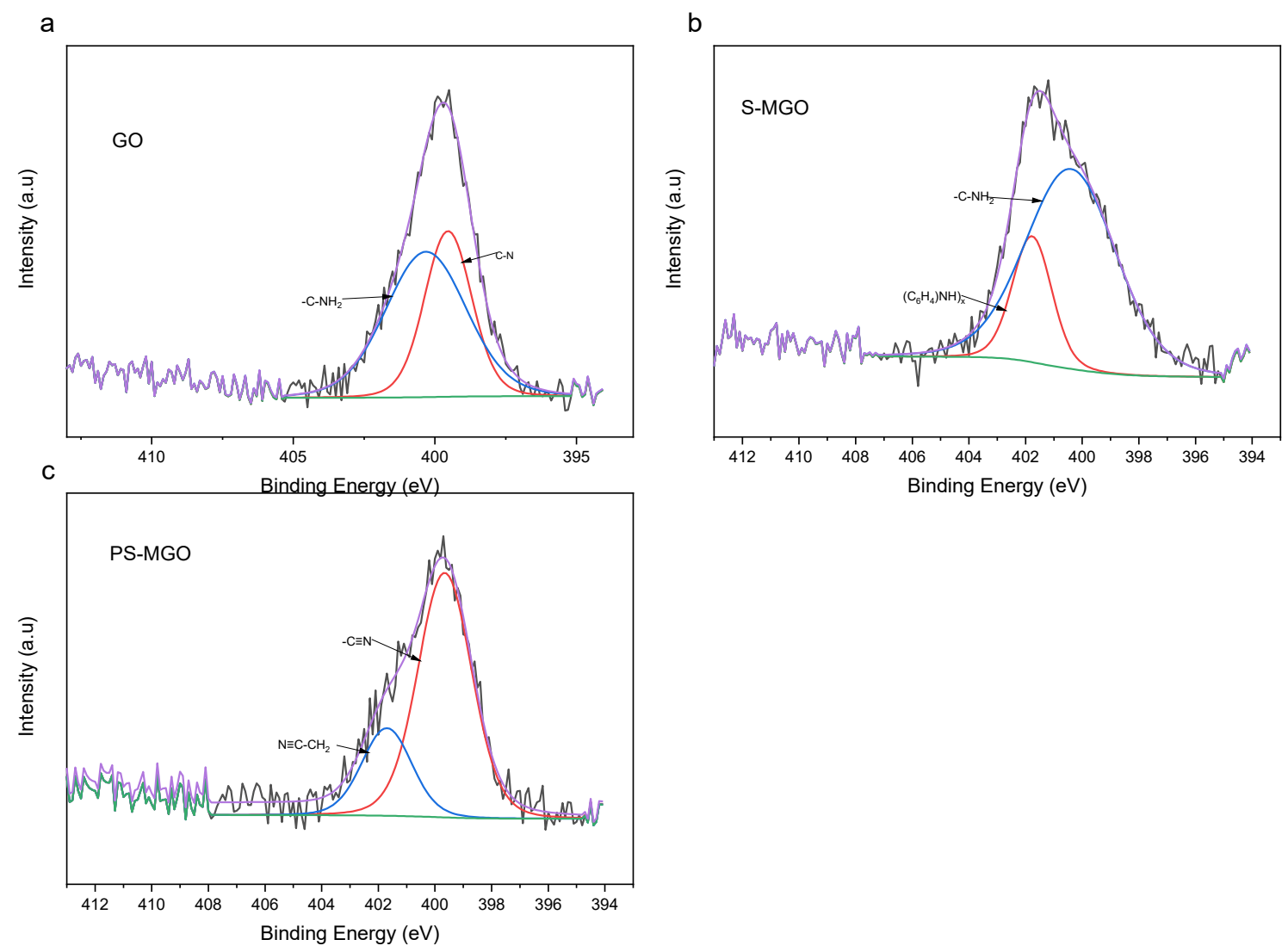

Figure 7.6. Deconvoluted XPS spectra in the N1s region for (a) MGO (b) S-MGO and (c) PS-MGO

\subsubsection{Morphology characterization and analysis}

The lamellar structure and morphology of pristine graphite, graphene oxide, modified graphene oxide and acid functionalized graphene oxide were investigated by FE-SEM as displayed in Figure 7.7. The cross-sectional view of SEM image is an effective way to evaluate expansion situation of graphene layers. The SEM cross-sectional images in Figure 7.7. shows the pristine graphite has compact and flat side structure, while the GO has flaky and wrinkled paper like structures due to the stacked lamellar were expanded by the oxide groups such as hydroxyl $(-\mathrm{OH})$, carboxyl $(-\mathrm{COOH})$ and carbonyl $(\mathrm{C}=\mathrm{O})$ groups. After modifying, a relative decreasing spacing between planers was observed in cross sectional image of MGO presenting in Figure 7.7. e), could be attributed to the oxygen groups contained in GO were partly removed or replaced by the (3-aminopropyl) tridmethoxy saline. Compare to the MGO, the much rougher lamellar edges were exhibited in P-MGO and S-MGO showed in Figure 7.7. g) and i), which are probably due to the existence of the sulfonic acid and phosphoric acid groups respectively. These acid groups have interactions with $-\mathrm{NH}_{2}$ and oxide groups, resulting the disorder of the 
layered structure. Cross sectional image of PS-MGO in Figure 7.7. k) presented a characteristic layered structure, indicating multiple functionalization effects of sulfanilic acid and phosphoric acid groups on MGO. The surface morphology characterization of $\mathrm{GO}, \mathrm{MGO}$ and functionalized GO are presented through the surface view. Compare to the pristine graphite, the GO and MGO exhibited relatively rougher and loose surface. A small number of cracks and pinoles can be observed in P-MGO and S-MGO which are shown in Figure 7.7. h) and i), suggesting the expanding inner structures.

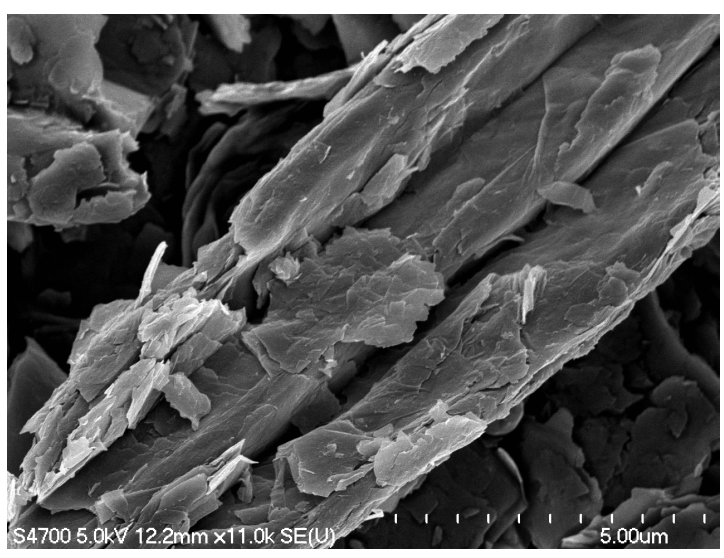

a

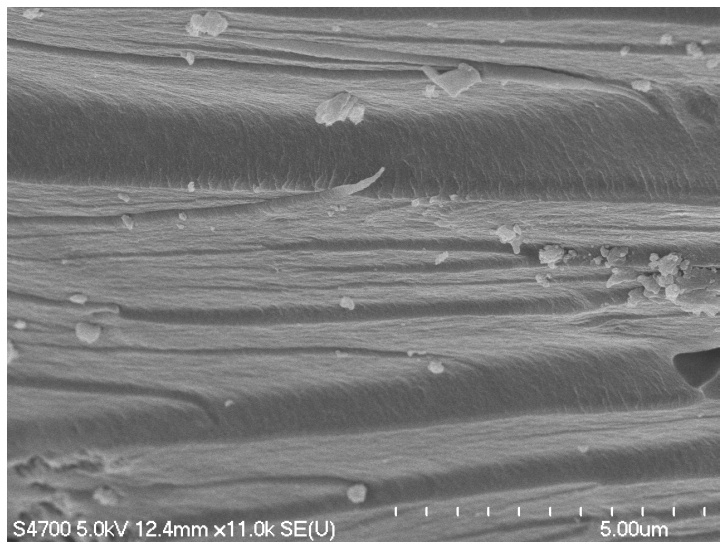

$\mathrm{c}$

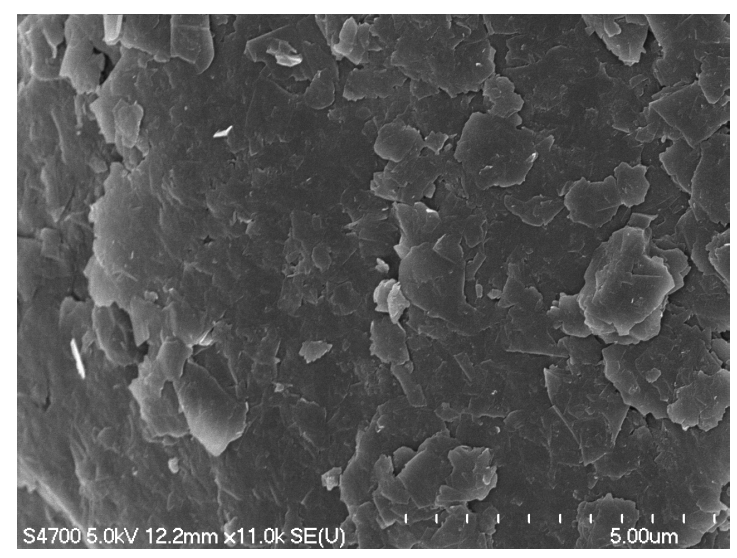

b

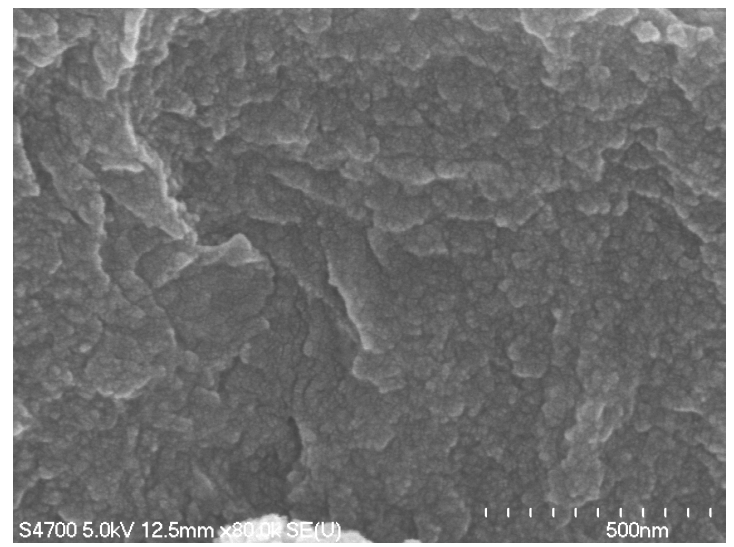

d 


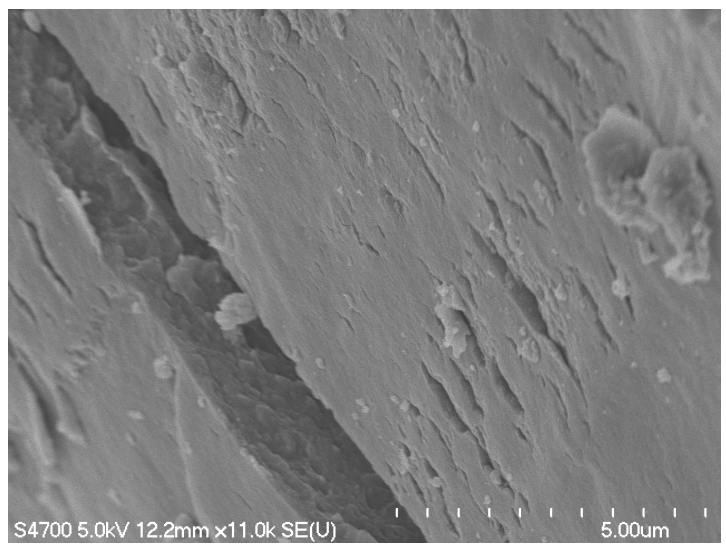

e

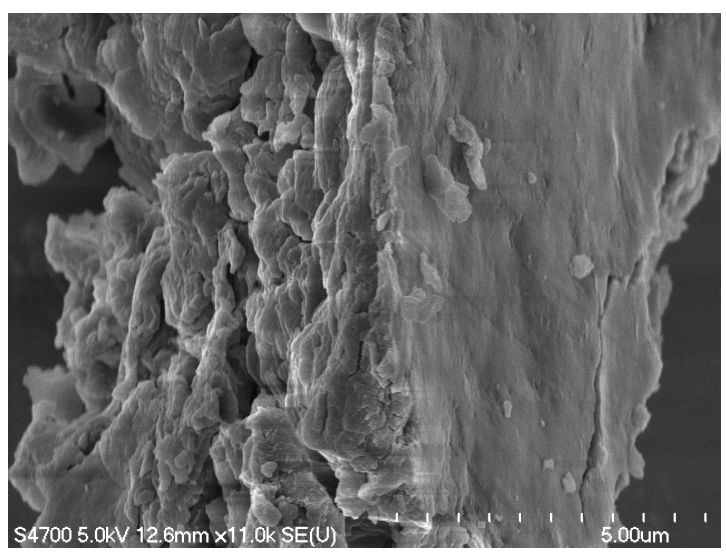

g

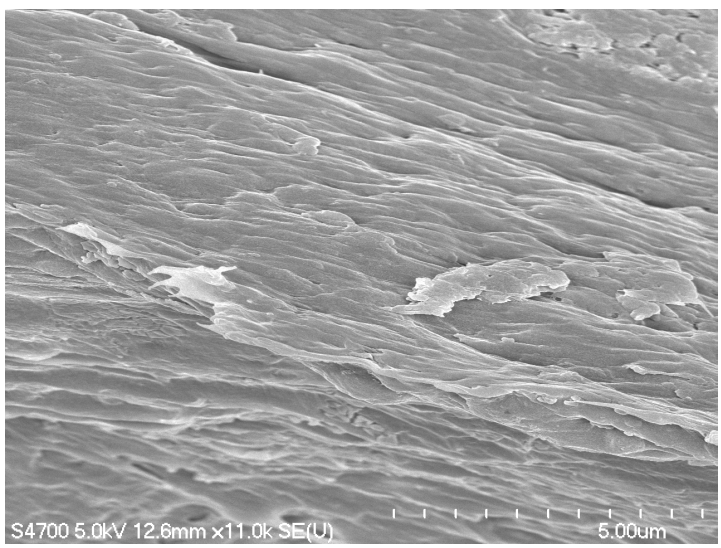

S47005.0kV $12.6 \mathrm{~mm} \times 11.0 \mathrm{k}$ SE(U)

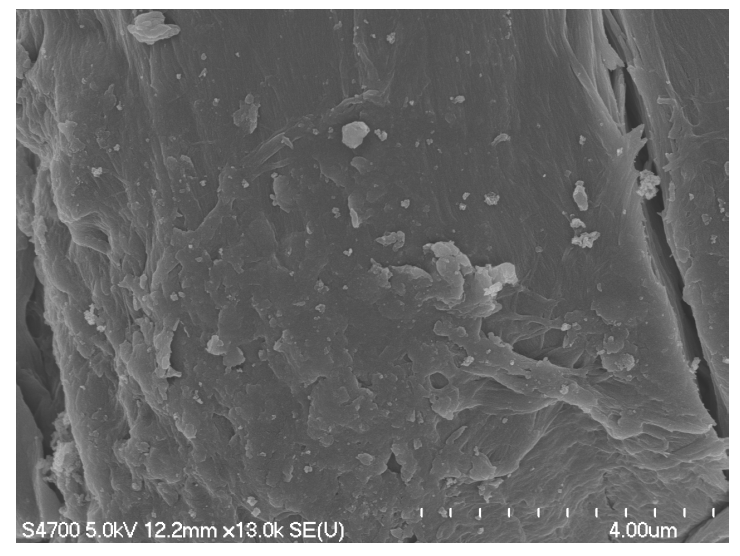

f

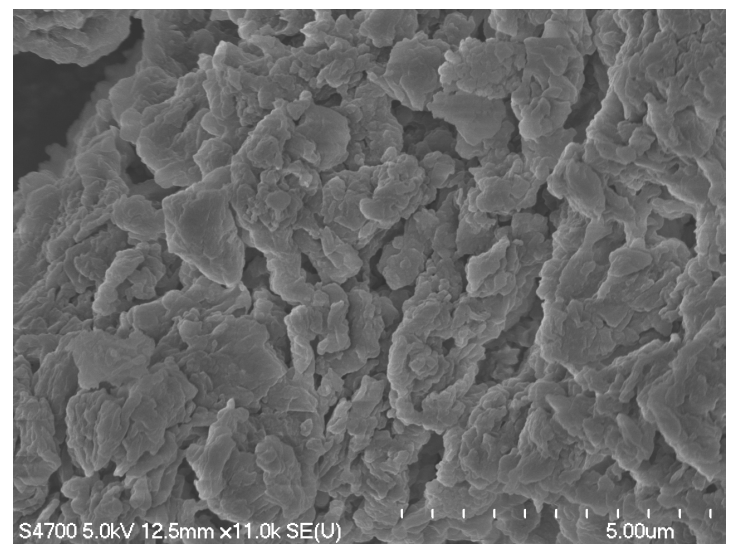

h

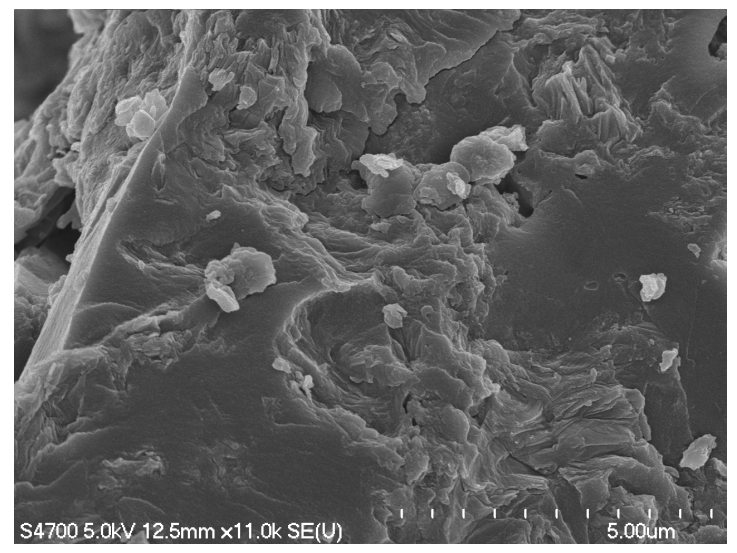




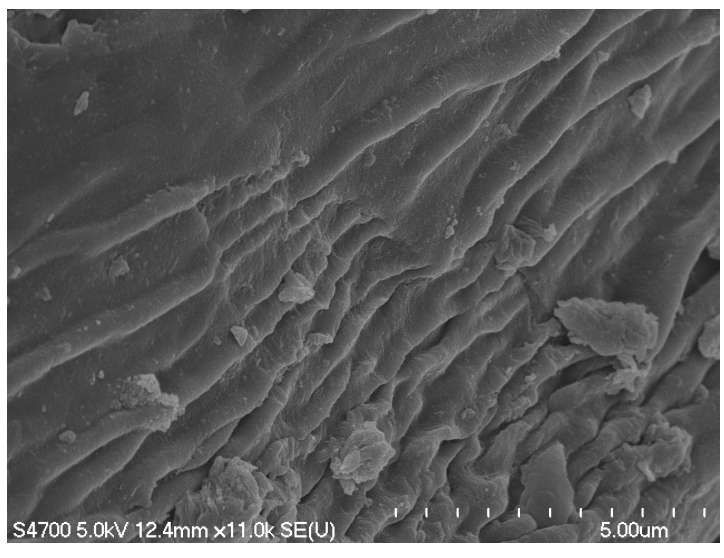

$\mathrm{k}$

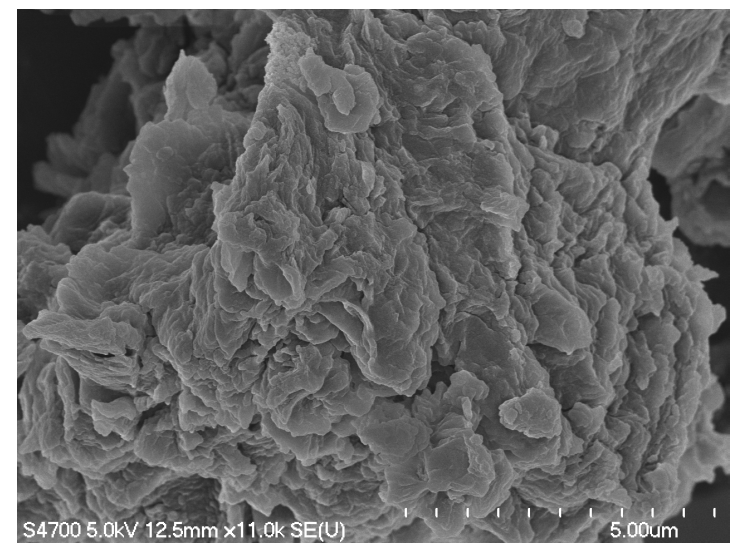

1

Figure 7.7. Cross-sectional FE-SEM images of a) graphite powder c) GO e) MGO g) PMGO i) S-MGO k) PS-MGO; SEM surface view of b) graphite powder d) GO f) MGO h) P-MGO j) S-MGO 1) PS-MGO

The morphology of raw PE-g-PS based CEM, S-MGO/CEM composite and PS$\mathrm{MGO} / \mathrm{CEM}$ composite membranes were also investigated by FE-SEM. The cross sectional and surface SEM images (Figure 7.8. a and b) of original CEM present a relatively smooth and dense structure without obvious defects. While the S-MGO/CEM composite membrane which showed in Figure 7.8. c) and d) are observed rougher and rugged surface. The surface view of $\mathrm{S}-\mathrm{MGO} / \mathrm{CEM}$ composite clearly reveal the additives well dispersed in different region of CEM matrix. Furthermore, the cross-sectional and surface images of PS-MGO/CEM composite membrane show more fillers are embedded firmly inside membrane, indicating good compatibility between PS-MGO and CEM matrix. This cohesion may come from the mutual interaction between fillers and matrix due to the (1) $\pi-\pi$ interaction between functional graphene oxides and CEM, (2) the hydrogen bonding interactions between $-\mathrm{PO}_{3} \mathrm{H},-\mathrm{SO}_{3} \mathrm{H}$ of $\mathrm{PS}-\mathrm{MGO}$ and $-\mathrm{SO}_{3} \mathrm{H}$ of CEM. Compared to S-MGO with single functional site, the GO with co-functional sites displayed favorable dispersion in membrane matrix, suggesting a better compatibility between co-functionalized GO and matrix were obtained. When PS-MGO as a filler, a hydrogen interaction between PS-MGO and CEM may be involved, which play the most important role in interfacial interactions. 


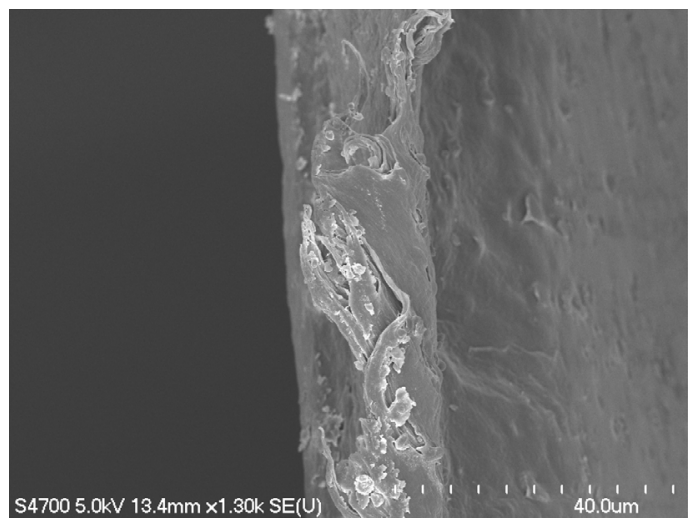

a

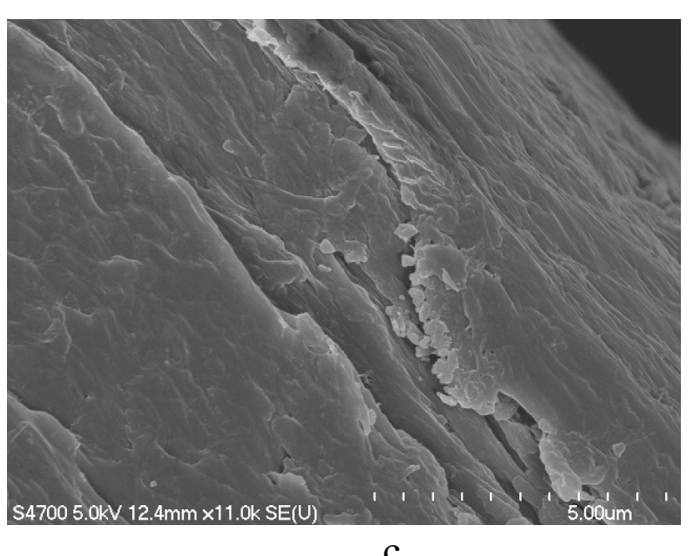

c

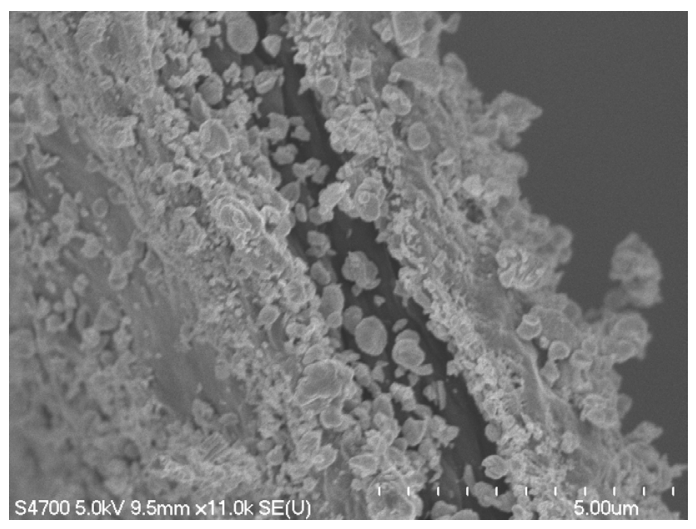

e

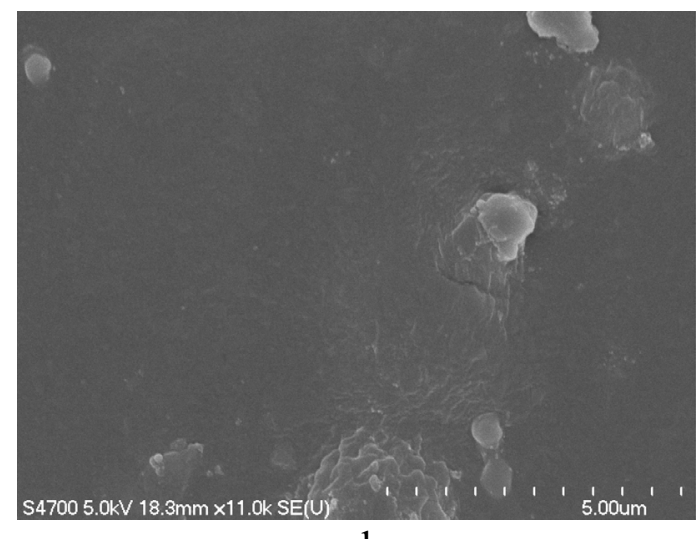

b
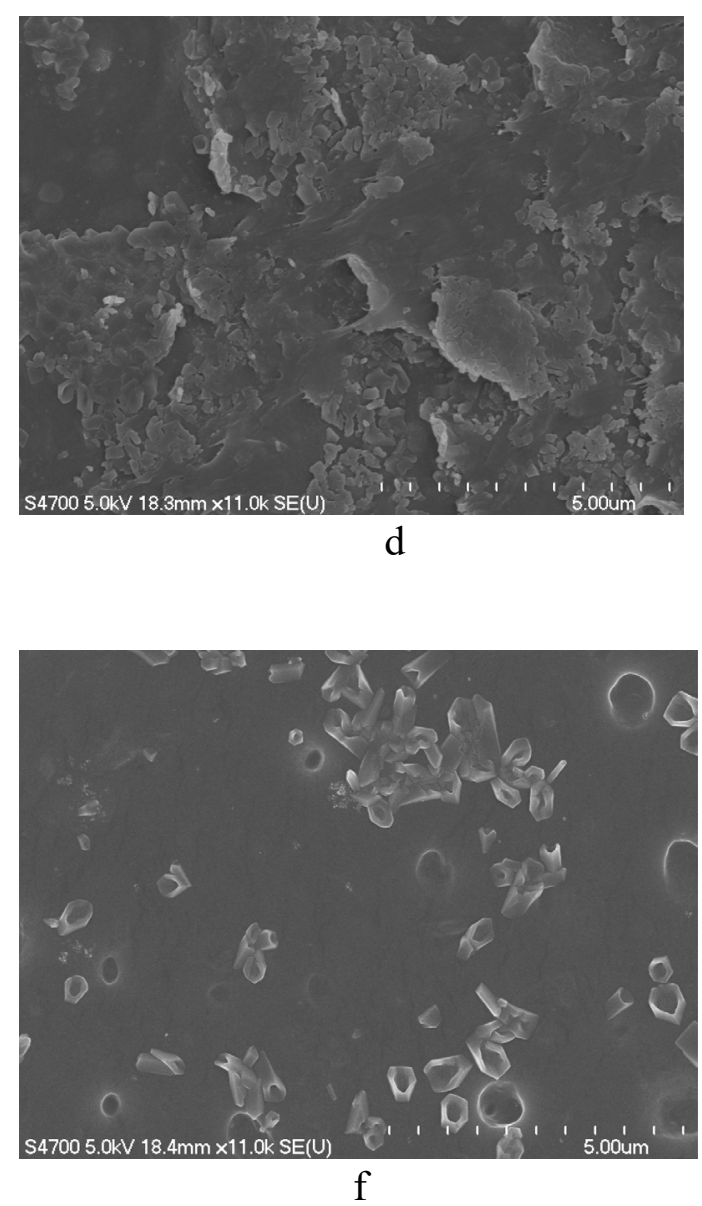

Figure 7.8. FE-SEM images of the cross section and the surface of ( $a$ and $b$ ) original CEM, (c and d) S-MGO/CEM composite and (e and f) PS/CEM 


\subsubsection{Mechanical properties of PS-MGO/CEM and S-MGO/CEM membrane composites}

The effect of fillers' types and amounts on mechanical properties of membrane composite are illustrated in Figure 7.8. and Figure 7.9. The Figure 7.8. presents that with the amounts of PS-MGO increasing, the maximum stress peak of composite is moving to higher point and the slope of stress-strain curve is getting steeper, reflecting the increasing tensile strength and tensile modulus. The increasing tensile strength and tensile modulus indicates that with the increasing additions of PS-MGO in matrix, the CEM composite would get harder, and have better deformation resistance against water flow. The strain-stress curve of $8 \% \mathrm{~S}-\mathrm{MGO}$ contained CEM composite has the same steeper extent as 8\% PS-MGO CEM's but lower stress peak value. This phenomenon would result that PS-MGO/CEM composite needs more force to be destructed but have the same elongation for unit applied force. Compare to the $8 \%$ addition of PS-MGO, the CEM composite with $6 \%$ addition of PS-MGO has larger elongation, indicating the CEM with $8 \%$ PS-MGO is stronger but not tougher than 6\% doped CEM.

The Figure 7.9. illustrates the effects of PS-MGO additions in CEM on DMA as a function of temperature. With the increasing additions of PS-MGO, the maximum value of storage modulus increased. The $8 \%$ PS-MGO/CEM composite has the largest storage modulus value in the temperature range of $30-150^{\circ} \mathrm{C} .8 \% \mathrm{~S}-\mathrm{MGO} / \mathrm{CEM}$ has less superiority on storage than that on tensile strength. It possesses higher storage modulus than $2 \%$ and $4 \%$ PS-MGO/CEM when temperature is below $120^{\circ} \mathrm{C}$, but lower than $6 \%$ PS-MGO/CEM composite. When functionalized GO were filled into CEM matrix, the $\pi$ $-\pi$ and hydrogen bonding were constructed, resulting a strong interfacial adhesion between them. The functionalized GO can be treated as a framework, which inhibits the chain motion of the CEM matrix when a force is applied to it.. 


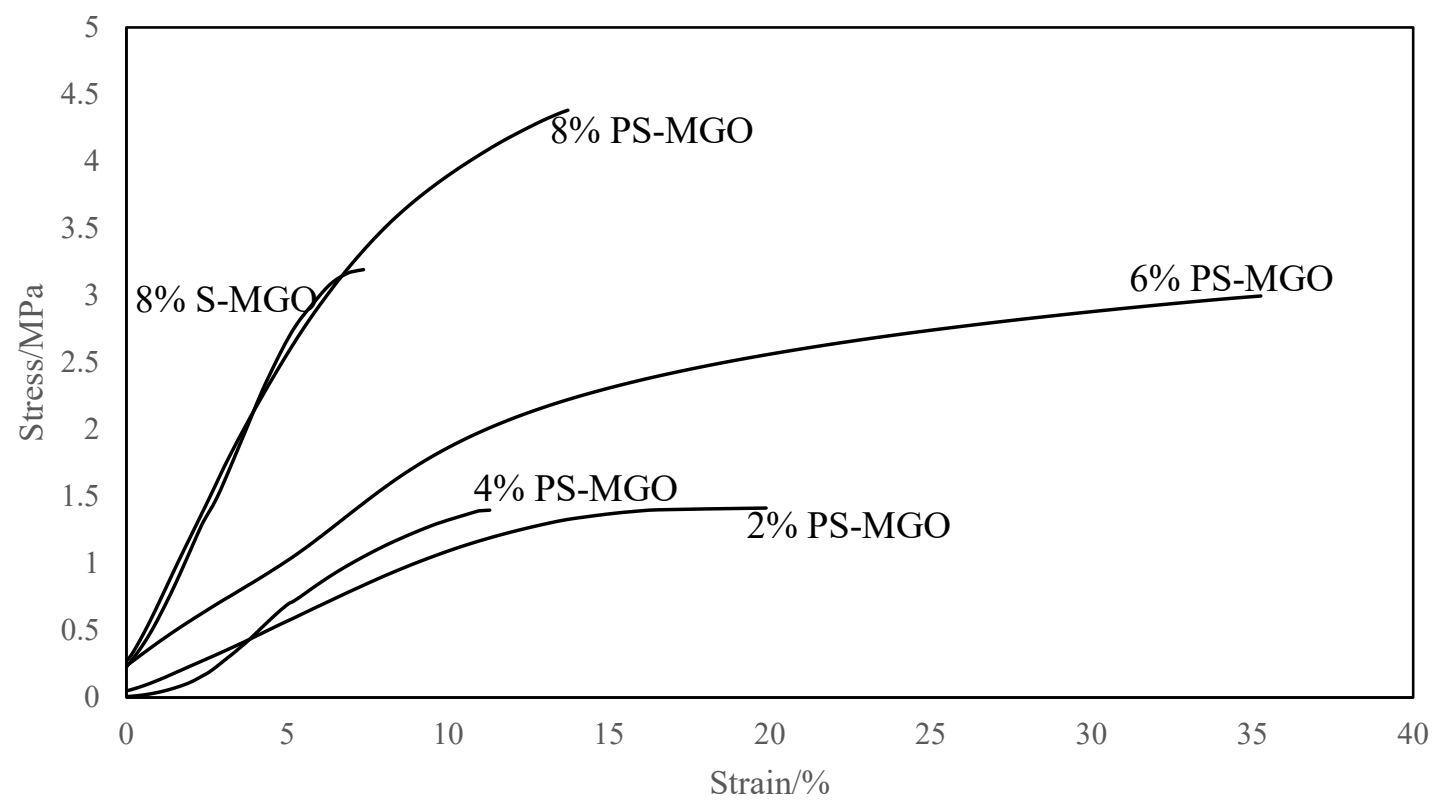

Figure 7.8. Stress-strain relationship of CEM composites with $8 \% \mathrm{~S}-\mathrm{MGO}$ and different amounts of PS-MGO

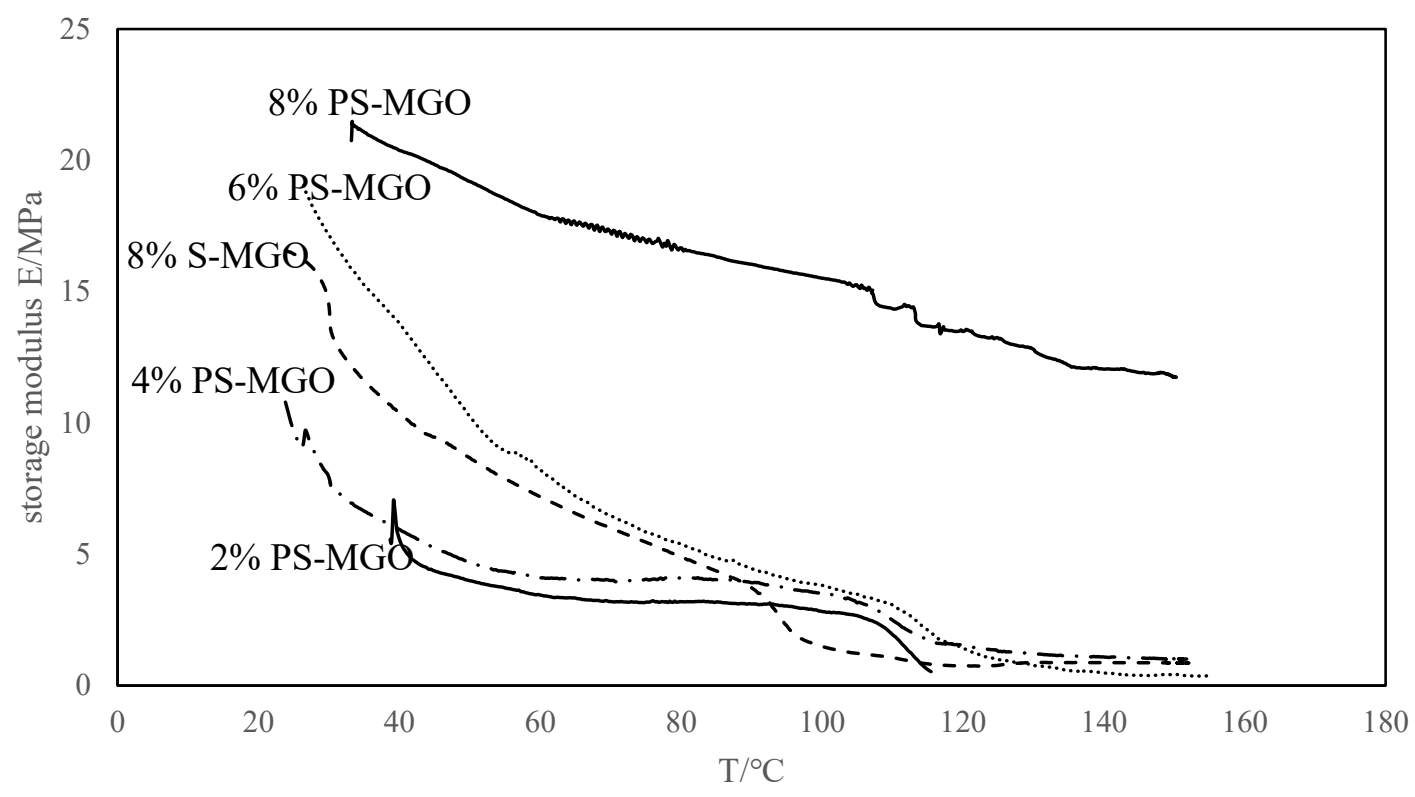


Figure 7.9. Storage-temperature relationship of CEM composites with $8 \% \mathrm{~S}-\mathrm{MGO}$ and different amounts of PS-MGO

\subsubsection{IEC and water uptake}

The mechanical properties of composite membranes are generally improved when membrane matrix is incorporated with non-ionic materials. However, the ionic conductivity and IEC may deteriorate due to block conductive channel. To address this serious issue, incorporation of functionalized $\mathrm{GO}$ in membrane matrix was applied, such as $-\mathrm{PO}_{3} \mathrm{H},-\mathrm{SO}_{3} \mathrm{H}$. To assess the water retention ability and ion transfer efficiency of membrane incorporated functionalized GO, IEC and water uptake are measured as the primary parameters. Figure 7.10., 7.11. presents those two parameters respectively for CEM composite with changing amounts of functionalized GO. IEC, as a parameter of evaluating ions conduction, is used for measuring density of hydrophilic functional groups presented in membrane. Figure 7.10. shows the IEC values for CEM composite membrane increased with both PS-MGO and S-MGO contents increasing. The IEC property of PS-MGO/CEM composite membranes are superior to S-MGO/CEM, having relatively high IEC value in $4 \%, 6 \%$ and $8 \%$ doping contents. Herein, the binary functionalized $\mathrm{GO}$ contains $-\mathrm{PO}_{3} \mathrm{H},-\mathrm{SO}_{3} \mathrm{H}$ has superior improving effect on CEM in compare with single $-\mathrm{SO}_{3} \mathrm{H}$ functionalized $\mathrm{GO}$, which attribute to the additional $-\mathrm{PO}_{3} \mathrm{H}$ groups provided by PS-MGO. Both functional groups $-\mathrm{PO}_{3} \mathrm{H},-\mathrm{SO}_{3} \mathrm{H}$ contained in PSMGO have ability to bridge hydrogen bonds with $-\mathrm{SO}_{3} \mathrm{H}$ in $\mathrm{CEM}$, but the potential difference is existent between them. Ions conduction and exchange will be facilitated due to the existence of potential difference.

The total water uptake depends on the density of hydrophilic functional groups and hydrogen bonding connected doping and matrix to capture bulk water. The water uptake of PS-MGO/CEM membrane composite increased with the increasing doping content in membrane matrix due to the hydrophilic nature of GO ( the presence of oxygen containing hydroxyl, epoxy, carbonyl and carboxyl groups) and the presence of multifunctional groups (carboxylic, sulfonic and phosphonic acid groups). Pristine CEM showed relatively low water uptake in compare with the doped CEM, which attribute to the insufficient hydrophilic groups' contents and relatively narrower transport path for water connected cations. The existence of oxygen-containing functional groups in GO make it well dispersed in water. Furthermore, when GO incorporates acid groups, such as $-\mathrm{PO}_{3} \mathrm{H},-\mathrm{SO}_{3} \mathrm{H}$, the expanded layer distance offers additional spacing for hydrated cation diffusion under vehicular mechanism. 


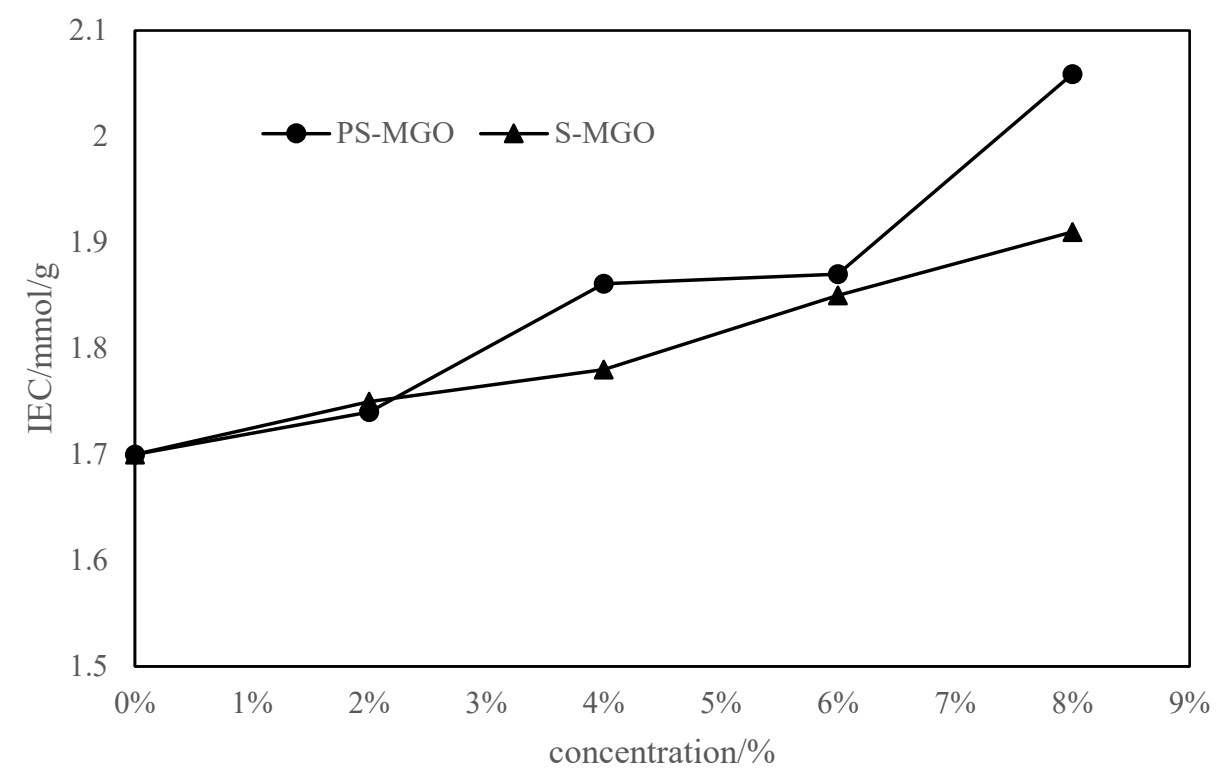

Figure 7.10. Comparation of IEC value of PS-MGO/CEM and S-MGO/CEM membrane composites

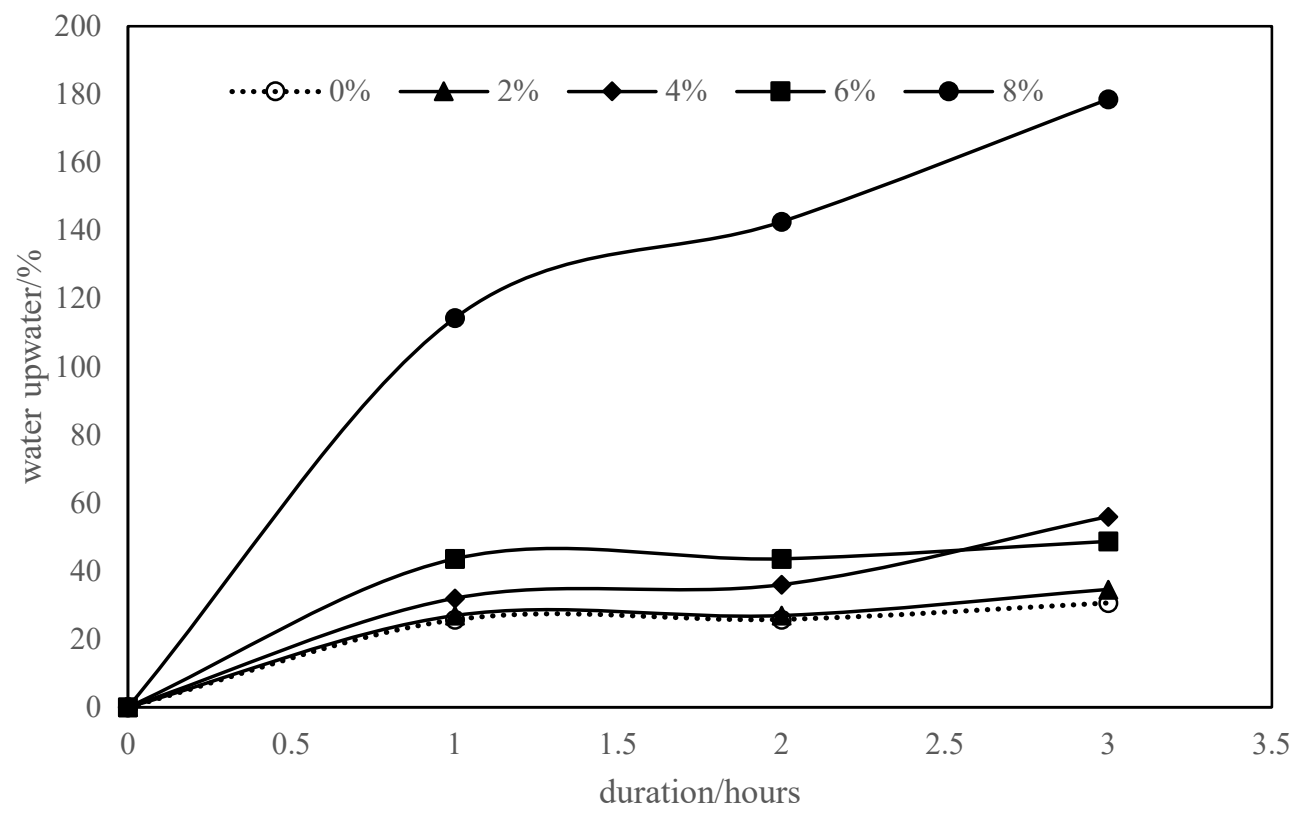

Figure 7. 11. Comparation of water uptake of pristine CEM and CEM doped different content of PS-MGO 


\subsubsection{Electrochemical impedance spectroscopy result and analysis}

\subsubsection{7.3.4.1 Principle of Electrochemical impedance spectroscopy}

Electrochemical impedance spectroscopy (EIS) is widely used to assess electrical properties and characterized electrochemical phenomena for conductive polymer immersed in electrolyte $[125,126]$. In EIS, the impedance of whole system including studying object and electrolyte surrounding it, was measured by inputting an alternating sinusoidal current with known amplitude ( $\mathrm{I}_{0}$ or $\left.\mathrm{U}_{0}\right)$, frequency range ( $f_{\text {initial }}$ and $\left.\mathrm{f}_{\text {final }}\right)$, and angular frequency $(\omega)$ across the sample, the responding signal such as amplitude $\left(\mathrm{I}_{(\mathrm{t})}\right.$ or $\left.\mathrm{U}_{(\mathrm{t})}\right)$ and phase shift $(\varphi)$ were monitored simultaneous [114]. The impedance $\mathrm{Z}$ can be calculated in accordance with Ohm's law:

$\mathrm{Z}=\frac{U(t)}{I(t)}$

Where the $\mathrm{U}(\mathrm{t})$ and $\mathrm{I}(\mathrm{t})$ are the voltage and alternating current as the function of time $\mathrm{t}$ respectively, and are defined as:

$\mathrm{U}(\mathrm{t})=U_{0} \sin \omega t=U_{0} e^{j \omega t}$

$\mathrm{I}(\mathrm{t})=I_{0} \sin (\omega t+\varphi)=I_{0} e^{j(\omega t+\varphi)}$

Where $\mathrm{U}(\mathrm{t})$ and $\mathrm{I}(\mathrm{t})$ are the voltage and alternating current at the certain time $t, \mathrm{U}_{0}$ and $\mathrm{I}_{0}$ are the voltage and alternating current without phase shift. $\phi$ is the phase shift. J is the imaginary unity $\left(\mathrm{j}^{2}=-1\right)$ and $\omega$ is the circular velocity $(1 \mathrm{rad} / \mathrm{s})$, which is defined as the function of frequency $\mathrm{f}$ :

$\omega=2 \pi \mathrm{f}$

the Ohm's law can be rewritten as:

$\mathrm{Z}(\omega)=\frac{U_{0} e^{j \omega t}}{I_{0} e^{j(\omega t+\varphi)}}=|Z| e^{-j \varphi}=|Z| \cos \varphi-j|Z| \sin \varphi$

Where the $e^{j \varphi}=\cos \varphi+j \sin \varphi$

When the simple CEM immersed in the electrolyte, besides the intrinsic membrane resistance (Figure 7.12. a), double layer and diffusion boundary layer resistance, which are related on the adjacent interfacial resistance [114], are included.

The CEM has high density of fixed negative charges attached on the based membrane (Figure 7.12. a), attracted the opposite charges within electrolyte to close. These opposite 
charges distribute on the membrane surface to form a double layer and produce an electrical double layer resistance for the interfacial ionic charger transfer from electrolyte through double layer to membrane (Figure $7.10 \mathrm{~b}$ ). The thickness of double layer is usually in the order of nanometers. In the bulk solution, current includes both positive and negative chargers. When current passes through CEM, the counter ions are captured and permitted through membrane whereas the co ions are rejected as the result of Donnan exclusion [120]. In this situation, the diffusion boundary layer (Figure 7.12. c) will generate due to the difference of ion transport amounts between bulk solution and the membrane surface. These layers typically have thicknesses in the order of micrometers, larger than the double layer.

The resistance of these different layers can be distinguished by EIS through the different responds for the input current or voltage signal with a range of frequencies [126]. At the high frequency, there is no phase shift between current and voltage, EIS outputs the respond signal of pure membranes. This single membrane is equal to a simple resistor in electrical circuit (Figure 7.12. d). When a current applied through the membrane, the resistance can be obtained by monitoring the voltage drop over membrane [120]. Typically, this resistance includes the total resistance of membrane and solution $(\mathrm{Rm}+\mathrm{s})$, while the membrane resistance can be extracted by removing the solution resistance.

Ztotal=Zmembrane + Zsolution

When the frequency decreased, the counter ions starts to pass through the double layer and membrane. The resistance $\left(\mathrm{R}_{\mathrm{dl}}\right)$ and capacitance $(\mathrm{C})$ of ions transport through double layer can be extracted due to the phase shift. In the equivalent electrical circuit, the ions transfer through double lay can be represented in parallel resistor and capacitor (Figure $7.10 \mathrm{e})$.

At the very low frequency range, the diffusion boundary layer origins from ions concentration gradients becomes visible, resulting ions transfer through membrane, double layer and diffusion boundary layer. The system responds a phase shift and phase angle with the applied signal at each frequency. The ions migrate over double layer and diffusion boundary layer is equivalent with a serial simple resistor and Warburg resistor, then parallel with a capacitor (Figure 7.12. f). The impedance can be expressed as

$$
\operatorname{Ztotal}(\omega)=\mathrm{R}_{m+s}+\frac{R}{1+\omega^{2} R^{2} C^{2}}-j \frac{\omega R^{2} C}{1+\omega^{2} R^{2} C^{2}}
$$

Where the $\mathrm{R}_{\mathrm{m}+\mathrm{s}}$ is the total resistance of membrane and solution, $\mathrm{R}$ is the electrical resistance when the current and voltage are in phase, and $\mathrm{C}$ is double layer capacitor. The contribution of Warburg component (Figure 7.12. f) is neglected for simplifying. Hence, the total impedance of CEM is constituted by real number $Z_{\text {real }}(\omega)$ and imaginary number $\mathrm{j} \mathrm{Z}_{\mathrm{img}}(\omega)$. When the angular frequency reaches high $(\omega \rightarrow \infty), Z_{\text {total }}$ is expressed as $R_{\mathrm{m}+\mathrm{s}}$. while the angular frequency goes down very low $(\omega \rightarrow 0), Z_{\text {total }}$ equals to $R_{m+s}+R$. It can 
be identified by the Nyquist plot with two intercepts for high frequency and low frequency.

a High frequency

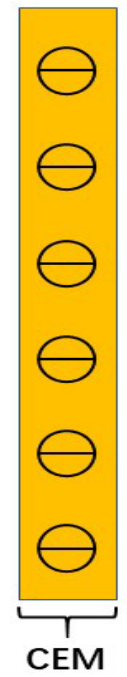

b Medium frequency

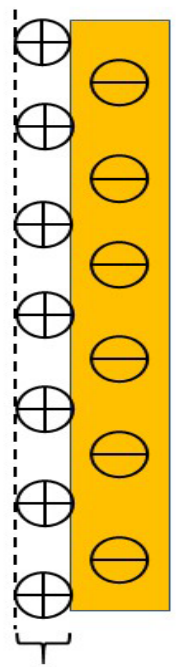

Double layer c Low frequency

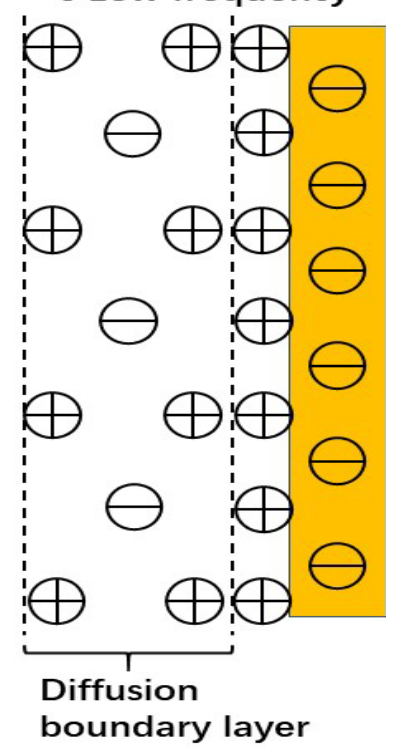

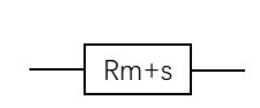

d Membrane+solution

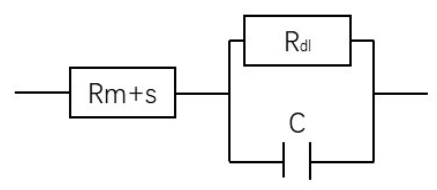

e Electrical double layer

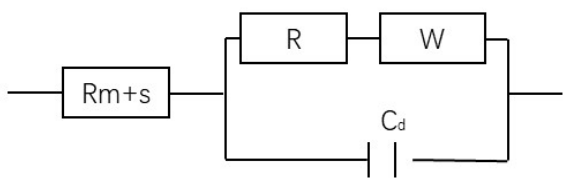

f Diffusion boundary layer

Figure 7.12. Phenomena of CEM and its adjacent layer in electrolyte. (a) CEM interface (b) double layer (c) diffusion boundary layer and equivalent circuit for CEM with solution.

\subsubsection{EIS measurement}

The EIS measurement were performed in the setup shown in Figure 7.13. by using Parstat 4000 potentiostat/galvanostat EIS analyzer. The cell was made of glass and consisted of two separated compartments with $50 \mathrm{ml}$ volume respectively. All the investigating membrane were equilibrated in electrolyte for $24 \mathrm{~h}$ before testing. The experiment was performed through four-electrode method. In four-electrode mode, the working and sense electrodes are coupled in one side, while the counter and reference electrodes are coupled in another side. Four-electrode setups measure potential along the B-D line in Figure 7.14, where there is researching objective at $\mathrm{C}$. The responds of applied current on solution and the barrier in that solution are measured. The advantage of this setup is accuracy for measuring the solution resistance and the resistance across the membrane 
material. The working and counter electrodes which constitute complete circuit are made of platinum. The sense and reference electrodes applied $\mathrm{Ag} / \mathrm{AgCl}$ electrodes, which were situated in tube filling with $3 \mathrm{M} \mathrm{KCl}$ and connected to the cell with Haber-Luggin capillaries. The potential drop and impedance over the membrane were monitored through these two electrodes. The both compartments were filled with $0.5 \mathrm{M} \mathrm{NaCl}$ solution.

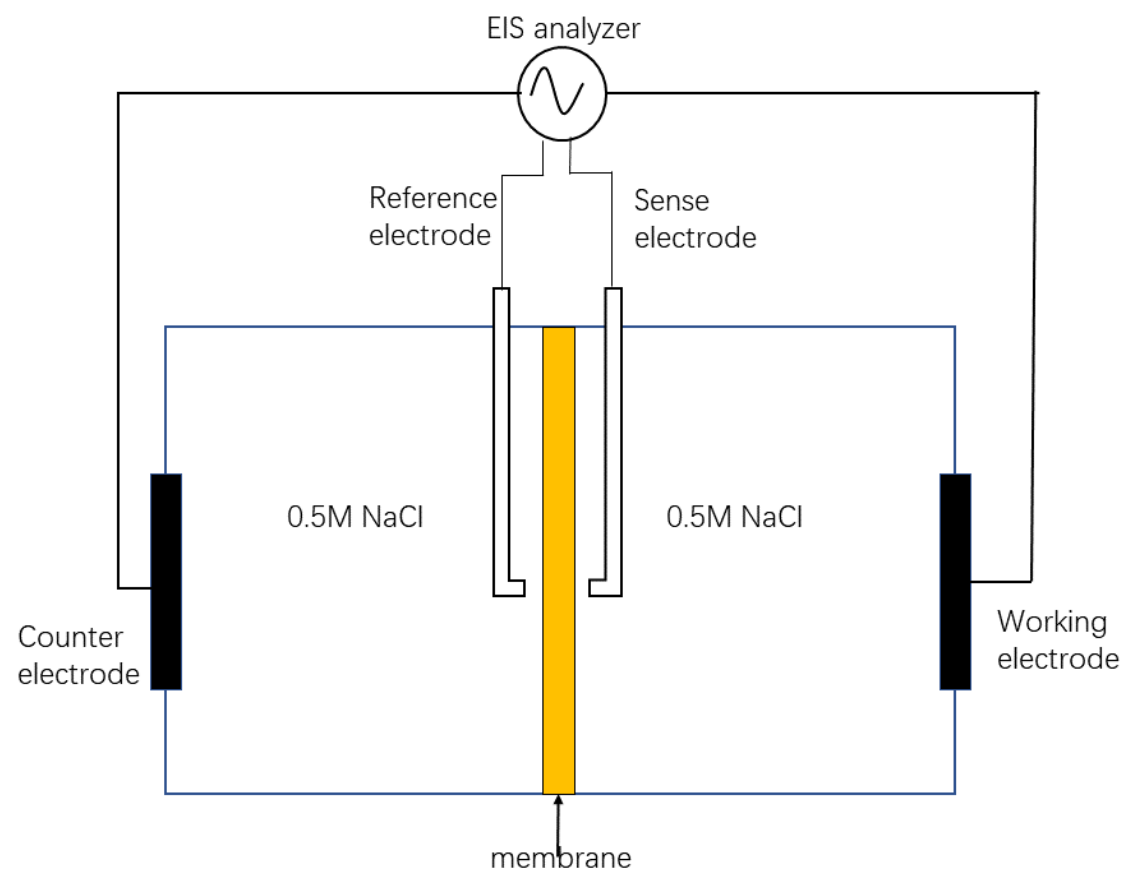

Figure 7.13. Schematic diagram of experimental setup for EIS

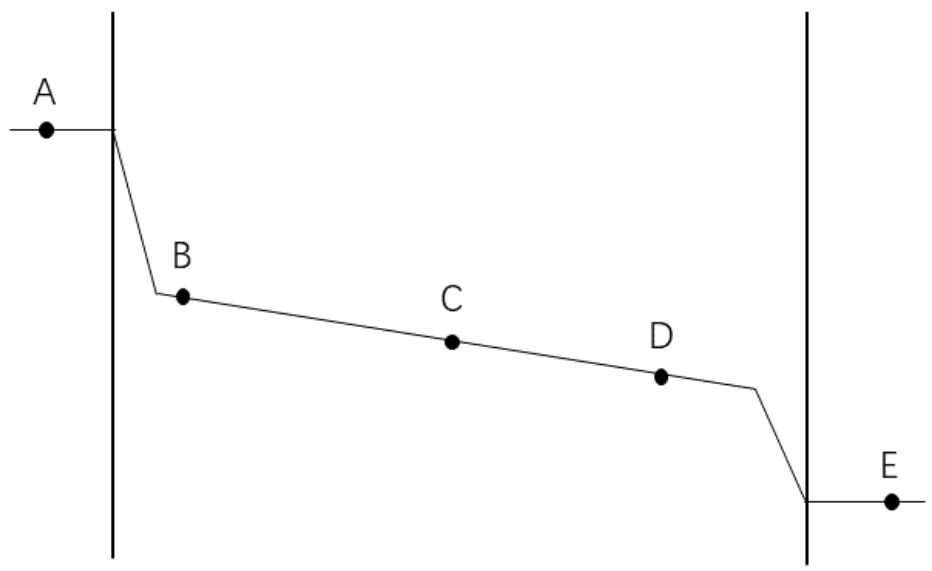


Figure 7.14. Potential drop map for signal across a whole cell. A is the working lead; B is the counter lead.

\subsubsection{EIS measure and analysis}

To compare the interfacial conductive behavior of composite membrane with varying doping amounts, the impedance data of investigated membrane were depicted by Nyquist plots as shown on Figure 7.15. As shown in the Nyquist plot of pristine CEM, CEM doped with 2\% PS-MGO, 4\% PS-MGO, 6\% PS-MGO, 8\% PS-MGO and 8\% S-MGO in the high frequency $(1000 \mathrm{~Hz})$, the membrane resistance value were $10.059 \Omega, 5.751 \Omega$, $3.973 \Omega, 3.863 \Omega, 2.368 \Omega, 4.200 \Omega$ respectively. These results indicate that membrane resistance decreased with the increasing amounts of PS-MGO doping. The $8 \% \mathrm{~S}-$ $\mathrm{MGO} / \mathrm{CEM}$ has higher membrane resistance compared with 8\% PS-MGO doped CEM. These results indicate that the dual functionalized GO offered more contribution in improving membrane conductivity compared with single functionalized GO, which is in good agreement with the IEC measurement. In addition, the charge transportation resistance which were decided by membrane resistance, double layer resistance and diffusion boundary layer resistance were characterized by the low frequency $(0.001 \mathrm{~Hz})$ impedance of Nyquist plot, showing the different tendency. The charge transportation resistance decreased with the amount of PS-MGO until $6 \%$ addition, the $8 \%$ PS-MGO doped CEM has higher resistance than 4\% and 6\% PS-MGO doped CEM. This phenomenon can be interpreted that CEM attached with high charged functional groups results strong polarization effect, which were reflected by larger diffusion boundary layer resistance. Therefore, the total resistance of $8 \% \mathrm{PS}-\mathrm{MGO} / \mathrm{CEM}$ is higher than $4 \%$ and 6\% PS-MGO doped CEM, though it has lower membrane resistance.

Ion conduction is an essential parameter for evaluating electrodialysis performance. The ionic conductivities of pure CEM and doped CEM are presented in Table 7.17. It is obviously that all the doped composite membrane exhibits higher ion transporting capability than pristine membrane. The increasement of ionic conductivity suggests that the introduction of functionalized GO make positive effect on conductivity enhancing and creates efficient ions transfer channel for CEM. The results are also in account with membrane resistance, higher amounts of doping, higher membrane conductivity. In addition, the CEM doped with dual functionalized GO is more conductive to ionic conductivity, compared to the CEM with single functionalized GO. This may be the result of synergistically promoting ion conduction of $-\mathrm{PO}_{3} \mathrm{H},-\mathrm{SO}_{3} \mathrm{H}$. Owing to the increasing water uptake capability, the increased ionic conductivity was suspected to vehicular mechanism contribution. The expansion spacing within doping and polymeric chains allow more hydrated ions transfer through membrane. Further, the electro potential difference between two functional groups drags more water connected counter ions through the membrane. 
The possible ion conduction mechanism of PS-MGO/CEM is depicted in Figure 7.16. The counter ions transportation is suggested to depend on the following ways: (1) The introduction of acid sites, constructed plentiful ionic clusters due to hydrogen bonding interaction with matrix. This creates extra charger sites for ions conduction and facilitate ions hopping via hopping mechanism, along with the formation and breakage of hydrogen bonding. (2) The introduction of doping with two different functional groups produces the potential difference, providing extra energy to drag water connected ions via vehicular mechanism. (3) For CEM doped with $-\mathrm{PO}_{3} \mathrm{H},-\mathrm{SO}_{3} \mathrm{H}$ functional groups, both can aggregate to form hydrophilic regions. The multiple interactions offer more ions transfer channel, making transportation more consecutive.

The relationship of ionic conductivities and temperature is presented in Figure 7.17. The ionic conductivities of pristine CEM and composite membrane increase with the elevating temperature due to the more water absorption in higher temperature facilitate ions transportation.

The activity energy deduced from the slop of temperature dependent conductivity curves, which is an important parameter to evaluate ions transfer efficient. Compare with pristine CEM, the doped CEM has lower activity energy and activity energy decreased with increasing doping amounts. These results indicated that dual-functionalized doping offers more ion conducting sites to provide more transfer pathways. Furthermore, the doped composite membrane possesses more consecutive ion transport channels due to the synergistic effect of $-\mathrm{PO}_{3} \mathrm{H},-\mathrm{SO}_{3} \mathrm{H}$.
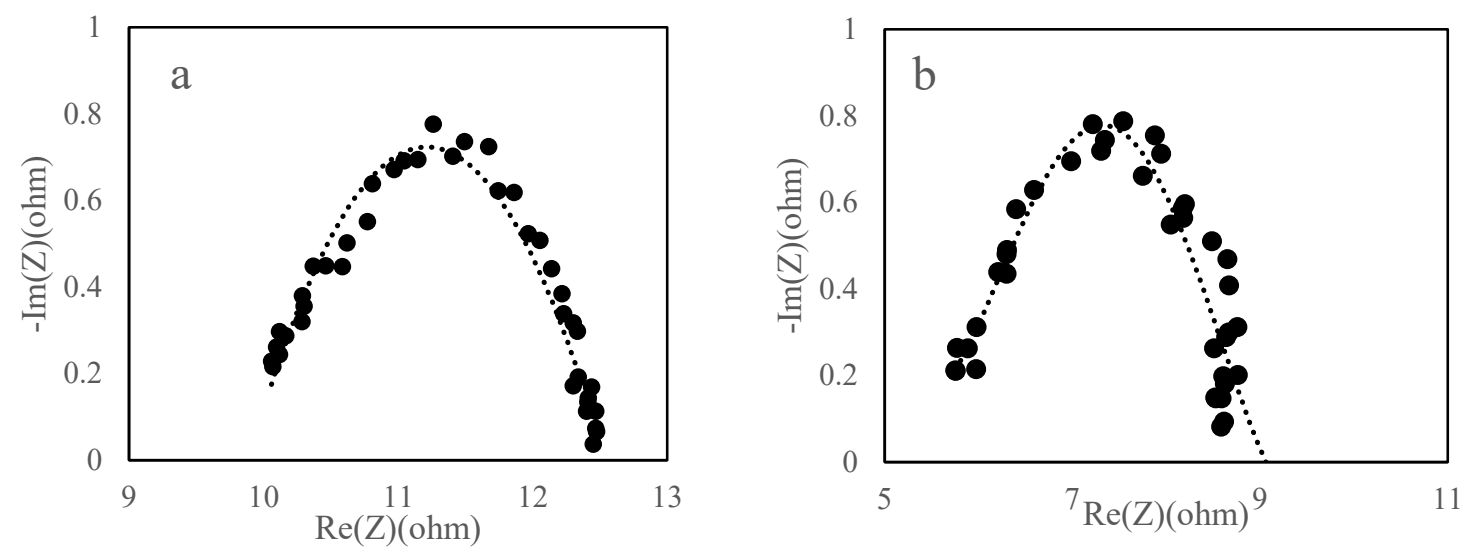

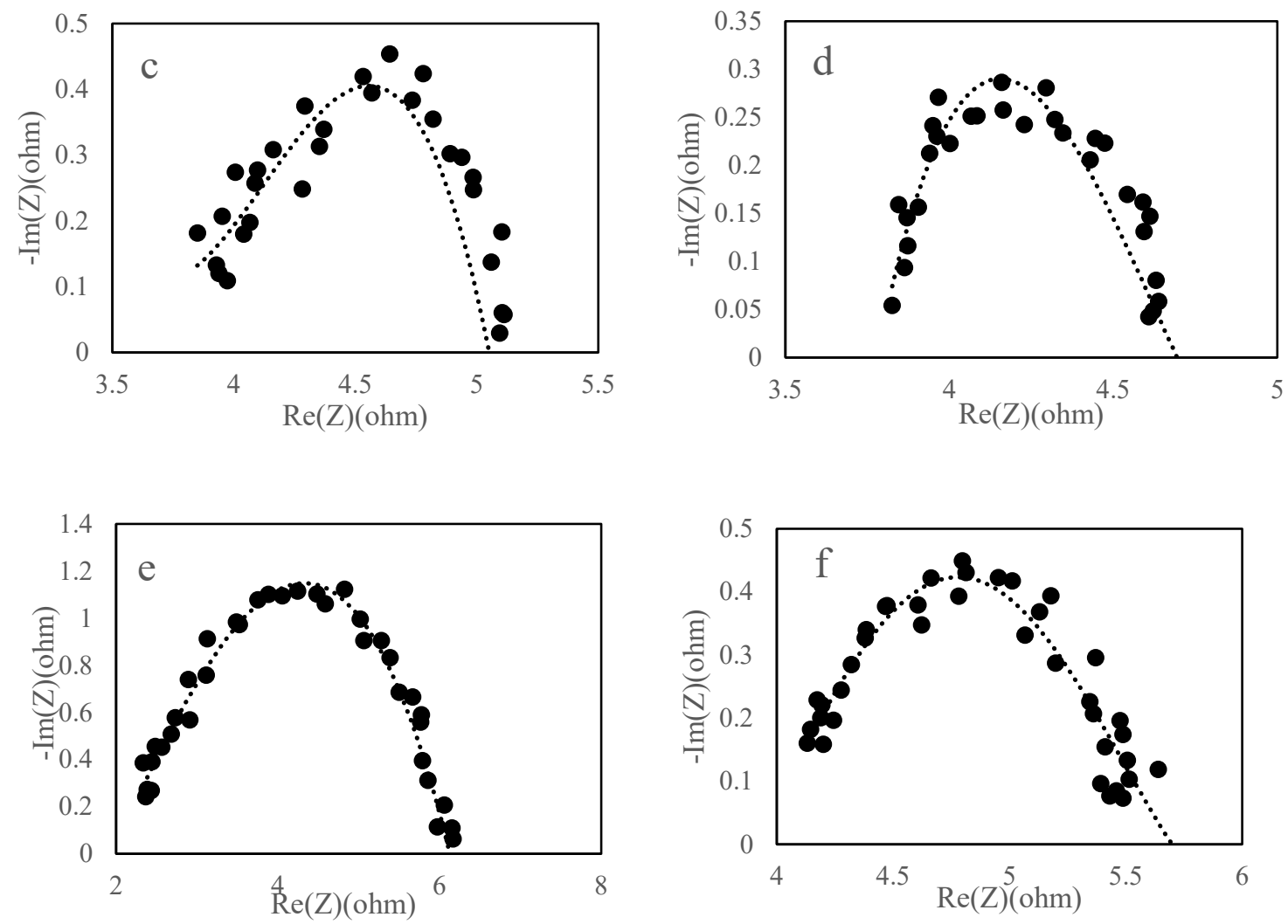

Figure 7.15. Nyquist plot obtained from EIS measurement, (a) pristine CEM, (b) $2 \%$ PSMGO/CEM membrane composite, (c) 4\% PS-MGO/CEM membrane composite, (d) 6\% PS-MGO/CEM membrane composite, (e) 8\% PS-MGO/CEM membrane composite, (f) $8 \% \mathrm{~S}-\mathrm{MGO} / \mathrm{CEM}$ membrane composite. $\operatorname{Re}(\mathrm{Z})$ is the real part, $\operatorname{Im}(\mathrm{Z})$ is the imaginary part of impedance $\mathrm{Z}$. 


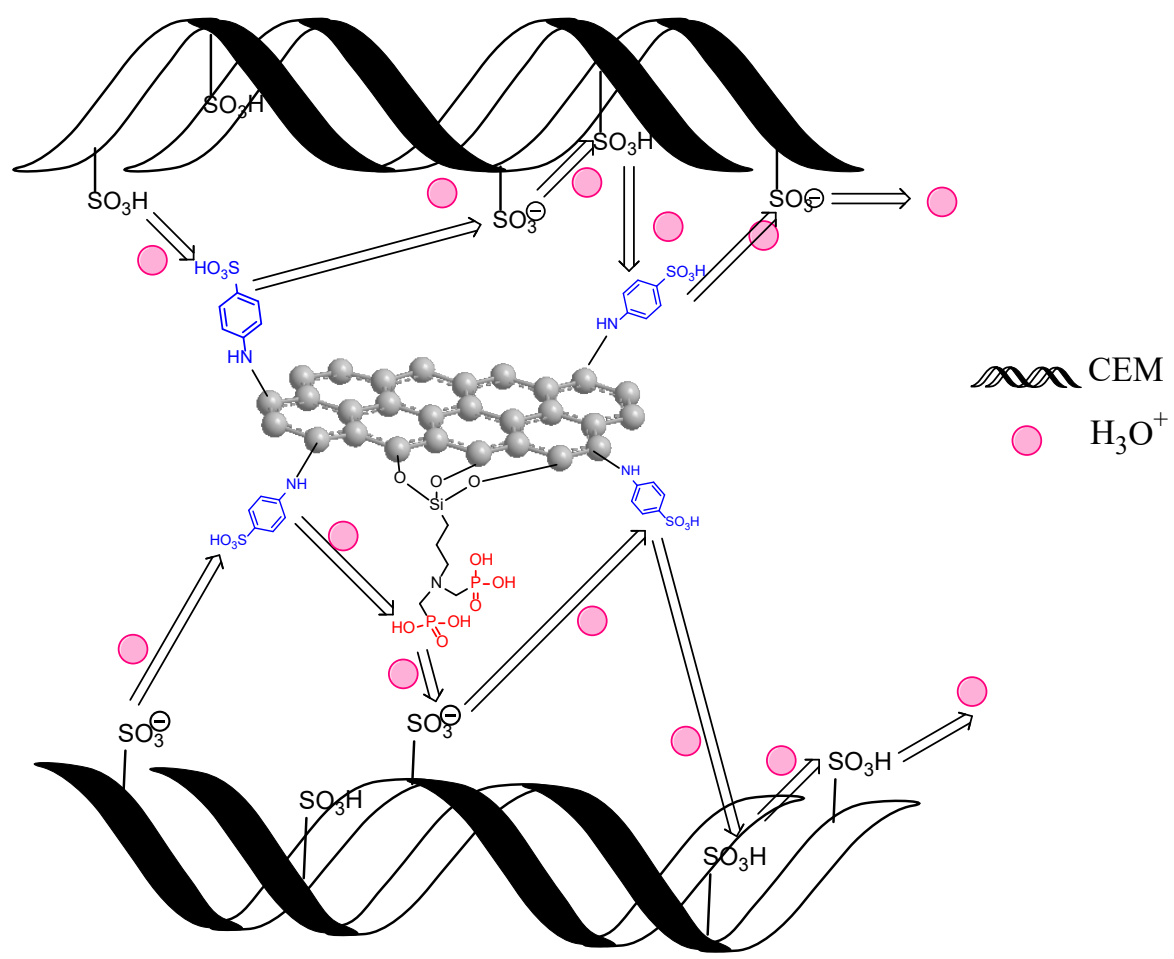

Figure 7.16. The ions conduction mechanism of composite membrane

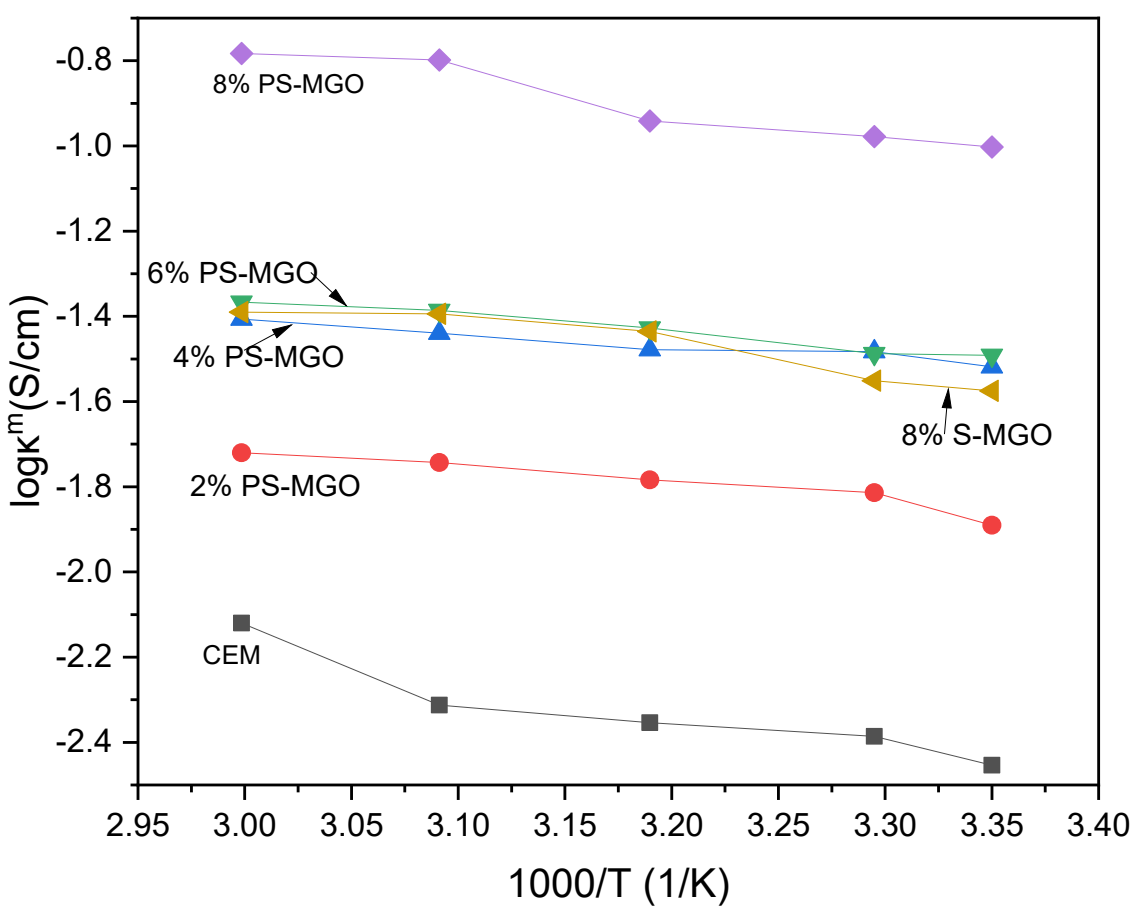


Figure 7.17. Temperature dependence of ionic conductivity

Table 7.1 Comparation of membrane conductivity of CEM contained different amounts of PS-MGO and S-MGO

\begin{tabular}{lll} 
Samples & Ionic conductivity $\left(\mathrm{S} \mathrm{cm}^{-1}\right)$ & Activation energy \\
\hline pristine CEM & 0.086 & 6.75 \\
\hline 2\% PS-MGO/CEM & 0.151 & 3.66 \\
\hline 4\% PS-MGO/CEM & 0.219 & 3.42 \\
\hline 6\% PS-MGO/CEM & 0.225 & 3.25 \\
\hline 8\% PS-MGO/CEM & 0.367 & 2.72 \\
\hline 8\% S-MGO/CEM & 0.207 & 4.82 \\
\hline
\end{tabular}

\subsection{Summary}

The raw graphite was oxidized through Hummers method, and then was modified by (3aminopropyl) trimethoxy silane to attach $-\mathrm{PO}_{3} \mathrm{H},-\mathrm{SO}_{3} \mathrm{H}$ as functional groups. The synthetic results indicated that layer spacing was expanded after modification, and furtherly extended after adding functional groups. The pristine CEM was doped with dual-functionalized GO and single functionalized GO respectively. It was found that functionalized GO doping greatly improved membrane performance, including mechanical properties, IEC ( $21 \%$ higher), water uptake capability ( $415 \%$ more) and membrane conductivity properties ( $326.7 \%$ higher). In addition, the properties enhancement was proportional with the amounts of doping. The results suggest that the improving effects of dual-functionalized GO on CEM is superior than the single functionalized GO does. Specifically, the coexistence of $-\mathrm{PO}_{3} \mathrm{H},-\mathrm{SO}_{3} \mathrm{H}$ in $\mathrm{GO}$ lead to the CEM possessed 7.8\% higher IEC, $77.29 \%$ higher membrane conductivity and $43.56 \%$ lower activation energy than that with single functionalized GO. This was due to 1) extra charger sites along doping interface for ions conduction, and 2) the potential difference between $-\mathrm{PO}_{3} \mathrm{H},-\mathrm{SO}_{3} \mathrm{H}$ provides extra energy to drag water connected ions through membrane. This study provides a new strategy on the design of high performance CEM with excellent mechanical property, high IEC, high conductivity and low membrane resistance for ion exchange membrane application. 


\section{Conclusion and recommendations for future work}

There are many properties we expect ion exchange membrane possess for the industrial applications, especially under harsh conditions, such as high salinity, high heavy metal concentrations and extreme $\mathrm{pH}$ value. The desired properties include high ion exchange capacity, which can enhance ions exchange and transportation efficiency; the excellent mechanical properties and dimensional stability, which can withstand the water flow impact; the superior ion conductivity ability, which can decrease power consumption. Unfortunately, these multiple properties cannot be accomplished at the same time, they have antagonistic relations.

The object of this research is to investigate the effects of based matrix, active monomers, crosslinking degree, chemical initiator, modified additives and reaction conditions on the multiple properties of ion exchange membrane. These properties include ion exchange capacity, mechanical properties, membrane resistance, electrochemical properties and thermal stability. To optimize these multiple properties and to provide promising method for their industrial, these parameter relations are qualitatively analyzed. The synthesis mechanisms were explored during this research.

The noteworthy conclusions were obtained in this research. The structure and crystallinity of inert matrix affect ion exchange capacity, mechanical properties, water uptake and thermal stability. The addition of active monomers has positive correction with ion exchange capacity. The crosslinking degree have positive correction with mechanical properties, but negative correction with ion exchange capacity and water uptake. Raising sulfonating temperature was confirmed to promote the sulfonation degree and make more functional groups attached on membrane. Styrene was found to dramatically improves ion exchange capacity and water uptake, attribute to the compatibilizer role styrene plays. With the addition of styrene, the phase separation between polyethylene and 4-Vinylbenzyl chloride can be improved.

This research also provides promising methods to improve mechanical property, membrane resistance and conductivity, which are three primary properties for ion exchange membrane. Through doping modified glass fiber in ion exchange membrane, the tensile strength and tensile modulus were enhanced. The 3-methacryloxypropyl trimethoxy silane was proved to have expected modification effect on glass fiber surface, making the glass fiber have strongest interface cohesiveness with membrane matrix. The pristine cation exchange membrane doped with dual-functional graphene oxide was found greatly improved membrane conductivity, ion exchange capacity and mechanical property. The main reasons for this improvement are the extra charger sites along doping interface for ion conduction and the potential difference between two different functional sites provides extra energy to drag water connected ions through membrane. 
The future of this research should focus on optimizing the perm selectivity of ion exchange membrane for monovalent ions and multivalent ions. In addition, the anion exchange membrane has different ions conduction mechanism with cation exchange membrane, the graphene oxide should be explored to modified with other functional groups to improve ions conductivity. 


\section{Reference List}

[1] Strathmann H. Membrane separation processes: current relevance and future opportunities. AIChE journal. 2001 May;47(5):1077-87.

[2] Jakobsen MR, Fritt-Rasmussen J, Nielsen S, Ottosen LM. Electrodialytic removal of cadmium from wastewater sludge. Journal of Hazardous Materials. 2004 Jan 30;106(23):127-32.

[3] Oehmen A, Viegas R, Velizarov S, Reis MA, Crespo JG. Removal of heavy metals from drinking water supplies through the ion exchange membrane bioreactor.

Desalination. 2006 Nov 20;199(1-3):405-7.

[4] Ibrahim Y, Abdulkarem E, Naddeo V, Banat F, Hasan SW. Synthesis of super hydrophilic cellulose-alpha zirconium phosphate ion exchange membrane via surface coating for the removal of heavy metals from wastewater. Science of The Total Environment. 2019 Nov 10;690:167-80.

[5] Kariduraganavar MY, Nagarale RK, Kittur AA, Kulkarni SS. Ion-exchange membranes: preparative methods for electrodialysis and fuel cell applications. Desalination. 2006 Oct 2;197(1-3):225-46.

[6] Peighambardoust SJ, Rowshanzamir S, Amjadi M. Review of the proton exchange membranes for fuel cell applications. International journal of hydrogen energy. $2010 \mathrm{Sep}$ 1;35(17):9349-84.

[7] Nagarale RK, Gohil GS, Shahi VK. Recent developments on ion-exchange membranes and electro-membrane processes. Advances in colloid and interface science. 2006 Feb 28;119(2-3):97-130.

[8] Zuo W, Zhang G, Meng Q, Zhang H. Characteristics and application of multiple membrane process in plating wastewater reutilization. Desalination. 2008 Mar 1;222(13):187-96.

[9] Yoshinobu Tanaka, editors. Ion Exchange Membranes Fundamentals and Applications, 2nd Edition. Japan, 2015

[10] Kurniawan TA, Chan GY, Lo WH, Babel S. Physico-chemical treatment techniques for wastewater laden with heavy metals. Chemical engineering journal. 2006 May 1;118(1-2):83-98.

[11] Eom TH, Lee CH, Kim JH, Lee CH. Development of an ion exchange system for plating wastewater treatment. Desalination. 2005 Aug 15;180(1-3):163-72. 
[12] Geise GM, Lee HS, Miller DJ, Freeman BD, McGrath JE, Paul DR. Water purification by membranes: the role of polymer science. Journal of Polymer Science Part B: Polymer Physics. 2010 Aug 1;48(15):1685-718.

[13] Akbal F, Camcı S. Copper, chromium and nickel removal from metal plating wastewater by electrocoagulation. Desalination. 2011 Mar 15;269(1-3):214-22.

[14] Hosseini SS, Bringas E, Tan NR, Ortiz I, Ghahramani M, Shahmirzadi MA. Recent progress in development of high performance polymeric membranes and materials for metal plating wastewater treatment: A review. Journal of water process engineering. 2016 Feb 1;9:78-110.

[15] Berezina NP, Kononenko NA, Dyomina OA, Gnusin NP. Characterization of ionexchange membrane materials: properties vs structure. Advances in colloid and interface science. 2008 Jun 22;139(1-2):3-28.

[16] Kumar V, Kumar P, Nandy A, Kundu PP. Crosslinked inter penetrating network of sulfonated styrene and sulfonated PVdF-co-HFP as electrolytic membrane in a single chamber microbial fuel cell. RSC Advances. 2015;5(39):30758-67.

[17] Wang KY, Chung TS. Fabrication of polybenzimidazole (PBI) nanofiltration hollow fiber membranes for removal of chromate. Journal of Membrane Science. 2006 Sep 15;281(1):307-15.

[18] Hwang GJ, Ohya H, Nagai T. Ion exchange membrane based on block copolymers. Part III: preparation of cation exchange membrane. Journal of Membrane Science. 1999 Apr 24;156(1):61-5.

[19] Gohil GS, Shahi VK, Rangarajan R. Comparative studies on electrochemical characterization of homogeneous and heterogeneous type of ion-exchange membranes. Journal of membrane science. 2004 Sep 1;240(1-2):211-9.

[20] Martí-Calatayud MC, Buzzi DC, García-Gabaldón M, Bernardes AM, Tenório JA, Pérez-Herranz V. Ion transport through homogeneous and heterogeneous ion-exchange membranes in single salt and multicomponent electrolyte solutions. Journal of Membrane Science. 2014 Sep 15;466:45-57.

[21] Pismenskaya ND, Pokhidnia EV, Pourcelly G, Nikonenko VV. Can the electrochemical performance of heterogeneous ion-exchange membranes be better than that of homogeneous membranes?. Journal of Membrane Science. 2018 Nov 15;566:5468.

[22] Banerjee S, Curtin DE. Nafion ${ }^{\circledR}$ perfluorinated membranes in fuel cells. Journal of fluorine chemistry. 2004 Aug 31;125(8):1211-6. 
[23] Hamad K, Kaseem M, Deri F, Ko YG. Mechanical properties and compatibility of polylactic acid/polystyrene polymer blend. Materials Letters. 2016 Feb 1;164:409-12.

[24] Tsuneda S, Saito K, Mitsuhara H, Sugo T. Novel ion - exchange membranes for electrodialysis prepared by radiation - induced graft polymerization. Journal of the Electrochemical Society. 1995 Nov 1;142(11): 3659.w

[25] Vogel C, Meier-Haack J. Preparation of ion-exchange materials and membranes. Desalination. 2014 Jun 2; 342:156-74.

[26] Hu G, Wang Y, Ma J, Qiu J, Peng J, Li J, Zhai M. A novel amphoteric ion exchange membrane synthesized by radiation-induced grafting $\alpha$-methylstyrene and $\mathrm{N}, \mathrm{N}$ dimethylaminoethyl methacrylate for vanadium redox flow battery application. Journal of membrane science. 2012 Jul 15; 407:184-92.

[27] Berezina NP, Komkova EN. A comparative study of the electric transport of ions and water in sulfonated cation-exchange polymeric membranes of the new generation. Colloid Journal. 2003 Jan 1;65(1):1-0.

[28] J. Liu, J. Yao, H. Wang, K. Chan, Fabrication of porous polymer particles with high anion exchange capacity by amination reaction in aqueous media, Green Chem. 8 (2006) 386-389.

[29] Y. Cao, X. Wang, K. Scott, The synthesis and characteristic of an anion conductive polymer membrane for alkaline anion exchange fuel cell, J. Power Sources 201 (2012) 226-230.

[30] Y. Xiong, J. Fang, Q.H. Zeng, Q.L. Liu, Preparation and characterization of crosslinked quaternuzed poly(vinyl alcohol) membranes for anion exchange membrane fuel cells, J. Membr. Sci. 311 (2008) 319-325.

[31] J. Qiao, J. Fu, R. Lin, J. Ma, J. Liu, Alkaline solid polymer electrolyte membranes based on structurally modified PVA/PVP with improved alkali stability, Polymer 51 (2010) 4850-4859.

[32] Y.Wan, B. Peppley, K.A.M. Creber, V.T. Bui, Anion-exchangemembranes composed of quaternized-chitosan derivates for alkaline fuel cells, J. Power Sources 195 (2010) 3785-3793.

[33] R. Martin, R. El Moussaoui, Solid alkaline fuel cell comprising ion exchange membrane, EU 1612874 A12004. 
[34] K. Mutsui, E. Tobita, K. Sugimoto, K. Kondo, A. Akimoto, Novel ion-exchange membranes having fluorocarbon backbone: preparation and stability, J. Appl. Polym. Sci. 32 (1986) 4137-4143.

[35] H.L.S. Salerno, F.L. Beyer, Y.A. Elabd, Anion exchange membranes derived from Nafion precursor for the alkaline fuel cel, J. Polym. Sci., Part B: Polym. Phys. 50 (2012) $552-562$.

[36] D. Valade, F. Boschet, S. Roulades, B. Ameduri, Preparation of solid alkaline fuel cell binders based on fluorinated poly (diallyl dimethylammonium chloride)s [Polygamic] or poly(chlorotrifluoroethylene-co-DADMAC) copolymeers, J. Polym. Sci., Part A: Polym. Chem. 47 (2009) 2043-2205.

[37] Lunkwitz K, Lappan U, Scheler U. Modification of perfluorinated polymers by highenergy irradiation. Journal of Fluorine Chemistry. 2004 Jun 1;125(6):863-73.

[38] W. Grot, Use of Nation perfluorosulfonic acid products as separators in electrolytic cells, Chem. Ing. Tech., 50 (1978) 299.

[39] Hamad K, Kaseem M, Deri F, Ko YG. Mechanical properties and compatibility of polylactic acid/polystyrene polymer blend. Materials Letters. 2016 Feb 1; 164:409-12.

[40] Varcoe JR, Slade RC. Prospects for alkaline anion - exchange membranes in low temperature fuel cells. Fuel cells. 2005 Apr;5(2):187-200.

[41] Liu H, Yang S, Wang S, Fang J, Jiang L, Sun G. Preparation and characterization of radiation-grafted poly (tetrafluoroethylene-co-perfluoropropyl vinyl ether) membranes for alkaline anion-exchange membrane fuel cells. Journal of membrane science. 2011 Mar 1;369(1-2):277-83.

[42] Ko BS, Sohn JY, Nho YC, Shin J. Radiolytic preparation of ETFE and PFA based anion exchange membranes for alkaline fuel cell. Nuclear Instruments and Methods in Physics Research Section B: Beam Interactions with Materials and Atoms. 2011 Nov 1;269(21):2509-13.

[43] Cardoso VF, Correia DM, Ribeiro C, Fernandes MM, Lanceros-Méndez S. Fluorinated polymers as smart materials for advanced biomedical applications. Polymers. 2018 Feb;10(2):161.

[44] Sherazi TA, Sohn JY, Lee YM, Guiver MD. Polyethylene-based radiation grafted anion-exchange membranes for alkaline fuel cells. Journal of membrane science. 2013 Aug 15;441:148-57. 
[45] Žitka J, Bleha M, Schauer J, Galajdová B, Paidar M, Hnát J, Bouzek K. Ion exchange membranes based on vinylphosphonic acid-co-acrylonitrile copolymers for fuel cells. Desalination and Water Treatment. 2015 Dec 18;56(12):3167-73.

[46] A. Elmidaoui, S. Belcadi, Y. Houdus, T. Cohen, C. Gavach, Perfluorinated anion exchange membranes: preparation and preliminary tests of dialysis, J. Polym. Sci. A 30 (1992) 1407.

[47] Sunaga K, Kim M, Saito K, Sugita K, Sugo T. Characteristics of porous anionexchange membranes prepared by cografting of glycidyl methacrylate with divinylbenzene. Chemistry of materials. 1999 Aug 16;11(8):1986-9.

[48] Stoica A. Preparation of nanostructured carbon films by Plasma Enhanced Chemical Vapor Deposition (Doctoral dissertation, Ph. D. Thesis, Masaryk University, Brno, Czech Republic).

[49] Wang L, Peng X, Mustain WE, Varcoe JR. Radiation-grafted anion-exchange membranes: the switch from low-to high-density polyethylene leads to remarkably enhanced fuel cell performance. Energy \& Environmental Science. 2019;12(5):1575-9.

[50] Wang L, Brink JJ, Liu Y, Herring AM, Ponce-González J, Whelligan DK, Varcoe JR. Non-fluorinated pre-irradiation-grafted (peroxidated) LDPE-based anion-exchange membranes with high performance and stability. Energy \& Environmental Science. 2017;10(10):2154-67.

[51] Sherazi TA, Sohn JY, Lee YM, Guiver MD. Polyethylene-based radiation grafted anion-exchange membranes for alkaline fuel cells. Journal of membrane science. 2013 Aug 15; 441:148-57.

[52] Nikolić S, Mojović L, Pejin D, Rakin M, Vučurović V. Improvement of ethanol fermentation of corn semolina hydrolyzates with immobilized yeast by medium supplementation. Food Technology and Biotechnology. 2009 Mar 26;47(1):83-9.

[53] Diaz MF, Barbosa SE, Capiati NJ. Polyethylene-polystyrene grafting reaction: effects of polyethylene molecular weight. Polymer. 2002 Aug 31;43(18):4851-8.

[54] Noonan KJ, Hugar KM, Kostalik IV HA, Lobkovsky EB, Abruña HD, Coates GW. Phosphonium-functionalized polyethylene: a new class of base-stable alkaline anion exchange membranes. Journal of the American Chemical Society. 2012 Oct 26;134(44):18161-4.

[55] Kostalik IV HA, Clark TJ, Robertson NJ, Mutolo PF, Longo JM, Abruña HD, Coates GW. Solvent processable tetraalkylammonium-functionalized polyethylene for 
use as an alkaline anion exchange membrane. Macromolecules. 2010 Aug 2;43(17):714750 .

[56] Mika AM, Childs RF, Dickson JM, McCarry BE, Gagnon DR. A new class of polyelectrolyte-filled microfiltration membranes with environmentally controlled porosity. Journal of membrane science. 1995 Dec 15;108(1-2):37-56.

[57] Peres AM, Pires RR, Oréfice RL. Evaluation of the effect of reprocessing on the structure and properties of low density polyethylene/thermoplastic starch blends. Carbohydrate polymers. 2016 Jan 20;136:210-5.

[58] Zhang M, Kim HK, Chalkova E, Mark F, Lvov SN, Chung TM. New polyethylene based anion exchange membranes (PE-AEMs) with high ionic conductivity. Macromolecules. 2011 Jul 14;44(15):5937-46.

[59] Schauer J, Brožová L. Heterogeneous ion-exchange membranes based on sulfonated poly (1, 4-phenylene sulfide) and linear polyethylene: preparation, oxidation stability, methanol permeability and electrochemical properties. Journal of membrane science. 2005 Mar 15;250(1):151-7.

[60] Sherazi TA, Sohn JY, Lee YM, Guiver MD. Polyethylene-based radiation grafted anion-exchange membranes for alkaline fuel cells. Journal of membrane science. 2013 Aug 15;441:148-57.

[61] Noonan KJ, Hugar KM, Kostalik IV HA, Lobkovsky EB, Abruña HD, Coates GW. Phosphonium-functionalized polyethylene: a new class of base-stable alkaline anion exchange membranes. Journal of the American Chemical Society. 2012 Nov 7;134(44):18161-4.

[62] Tam CM, Dal-Cin M, Guiver MD. Polysulfone membranes. IV. Performance evaluation of Radel A/PVP membranes. Journal of membrane science. 1993 Mar $25 ; 78(1-2): 123-34$.

[63] Kerres J, Ullrich A, Hein M, Gogel V, Friedrich KA, Jörissen L. Cross - linked polyaryl blend membranes for polymer electrolyte fuel cells. Fuel Cells. 2004 Apr;4(1 2):105-12.

[64] Kerres J, Ullrich A, Hein M. Preparation and characterization of novel basic polysulfone polymers. Journal of Polymer Science Part A: Polymer Chemistry. 2001 Sep 1;39(17):2874-88.

[65] J. Kerres, W. Cui, G. Eigenberger, D. Bevers, W. Schnurnberger, A. Fischer and H. Wendt, in Proc. 1 lth Hydrogen Conference, T.N. Veziroglu, C.J. Winter, J.E Baser and G. Kreysa, eds., Stuttgart, Germany, June 23-28, 1996, p. 1951. 
[66] J. Kerres, W. Cui and S. Reichle, New sulfonated engineering polymers via the metalation route. I. Sulfonated poly(ethersolfone) PSU Udel via metalation-sulfinationoxidation; J. Polym. Sci., Part A:Polym. Chem., 34 (1996) 2421.

[67] Mitsukami Y, Donovan MS, Lowe AB, McCormick CL. Water-soluble polymers. 81. Direct synthesis of hydrophilic styrenic-based homopolymers and block copolymers in aqueous solution via RAFT. Macromolecules. 2001 Mar 27;34(7):2248-56.

[68] Zhou T, Shao R, Chen S, He X, Qiao J, Zhang J. A review of radiation-grafted polymer electrolyte membranes for alkaline polymer electrolyte membrane fuel cells. Journal of Power Sources. 2015 Oct 20;293:946-75.

[69] Tsuneda S, Saito K, Mitsuhara H, Sugo T. Novel Ion - Exchange Membranes for Electrodialysis Prepared by Radiation - Induced Graft Polymerization. Journal of the Electrochemical Society. 1995 Nov 1;142(11):3659-63.

[70] Ko BS, Sohn JY, Nho YC, Shin J. Radiolytic preparation of ETFE and PFA based anion exchange membranes for alkaline fuel cell. Nuclear Instruments and Methods in Physics Research Section B: Beam Interactions with Materials and Atoms. 2011 Nov $1 ; 269(21): 2509-13$.

[71] Varcoe JR, Slade RC. Prospects for alkaline anion - exchange membranes in low temperature fuel cells. Fuel cells. 2005 Apr;5(2):187-200.

[72] Elmidaoui A, Belcadi S, Houdus Y, Cohen T, Gavach C. Perfluorinated anion exchange membranes: preparation and preliminary tests of dialysis. Journal of Polymer Science Part A: Polymer Chemistry. 1992 Jun;30(7):1407-12.

[73] Roualdes S, Kourda N, Durand J, Pourcelly G. Plasma-grafted PVDF polymers as anion exchange membranes for the electrotransport of Cr (VI). Desalination. 2002 Sep $10 ; 146(1-3): 273-8$.

[74] Stone C, Steck AE, Choudhury B, inventors; Ballard Power Systems Inc, assignee. Graft polymeric membranes and ion-exchange membranes formed therefrom. United States patent US 6,723,758. 2004 Apr 20.

[75] Lakeman JB, Rose A, Pointon KD, Browning DJ, Lovell KV, Waring SC, Horsfall JA. The direct borohydride fuel cell for UUV propulsion power. Journal of power sources. 2006 Nov 22;162(2):765-72.

[75] Barner L, Quinn JF, Barner-Kowollik C, Vana P, Davis TP. Reversible additionfragmentation chain transfer polymerization initiated with $\gamma$-radiation at ambient temperature: an overview. European Polymer Journal. 2003 Mar 1;39(3):449-59. 
[76] Çaykara T, Alaslan ŞŞ, Gürü M, Bodugöz H, Güven O. Preparation and characterization of poly (isobutyl methacrylate) microbeads with grafted amidoxime groups. Radiation Physics and Chemistry. 2007 Oct 1;76(10):1569-76.

[77] Nishiyama SY, Saito K, Saito K, Sugita K, Sato K, Akiba M, Saito T, Tsuneda S, Hirata A, Tamada M, Sugo T. High-speed recovery of antimony using chelating porous hollow-fiber membrane. Journal of Membrane Science. 2003 Apr 1;214(2):275-81.

[78] Gohil GS, Nagarale RK, Binsu VV, Shahi VK. Preparation and characterization of monovalent cation selective sulfonated poly (ether ether ketone) and poly (ether sulfone) composite membranes. Journal of colloid and interface science. 2006 Jun 15;298(2):84553.

[79] Naguib HF, Aly RO, Sabaa MW, Mokhtar SM. Gamma radiation induced graft copolymerization of vinylimidazole-acrylic acid onto polypropylene films. Polymer testing. 2003 Oct 1;22(7):825-30.

[80] Kostov GK, Turmanova SC. Radiation - initiated graft copolymerization of 4 vinylpyridine onto polyethylene and polytetrafluoroethylene films and anion - exchange membranes therefrom. Journal of applied polymer science. 1997 May 23;64(8):1469-75.

[81] Chung TC. Synthesis of functional polyolefin copolymers with graft and block structures. Progress in Polymer Science. 2002 Feb 28;27(1):39-85.

[82] Zhang XM, Elkoun S, Ajji A, Huneault MA. Oriented structure and anisotropy properties of polymer blown films: HDPE, LLDPE and LDPE. Polymer. 2004 Jan $31 ; 45(1): 217-29$.

[83] Van Cauter K, Van Speybroeck V, Vansteenkiste P, Reyniers MF, Waroquier M. Ab Initio Study of Free - Radical Polymerization: Polyethylene Propagation Kinetics. ChemPhysChem. 2006 Jan 16;7(1):131-40.

[84] Horsfall JA, Lovell KV. Synthesis and characterisation of sulfonic acid-containing ion exchange membranes based on hydrocarbon and fluorocarbon polymers. European polymer journal. 2002 Aug 31;38(8):1671-82.

[85] Espiritu R, Mamlouk M, Scott K. Study on the effect of the degree of grafting on the performance of polyethylene-based anion exchange membrane for fuel cell application. international journal of hydrogen energy. 2016 Jan 12;41(2):1120-33.

[86] Hwang ML, Choi J, Woo HS, Kumar V, Sohn JY, Shin J. Poly (vinylbenzyl sulfonic acid)-grafted poly (ether ether ketone) membranes. Nuclear Instruments and Methods in Physics Research Section B: Beam Interactions with Materials and Atoms. 2014 Feb 15;321:59-65. 
[87] Tongwen X, Jung-Je W, Seok-Jun S, Seung-Hyeon M. In situ polymerization: a novel route for thermally stable proton conductive membranes. J Membr Sci 2008;325:209

[88] Kreuer KD, Paddison SJ, Spohr E, Schuster M. Transport in proton conductors for fuel-cell applications: simulations, elementary reactions, and phenomenology. Chem Rev 2004; 104:4637-78.

[89] Deluca NW, Elabd YA. Polymer electrolyte membranes for the direct methanol fuel cell: a review. J Polym Sci Part B Polym Phys 2006;44:220-13.

[90] Grew KN, Chiu WK. A dusty fluid model for predicting hydroxyl anion conductivity in alkaline anion exchange membranes. Journal of the electrochemical Society. 2010 Jan 4;157(3):B327.

[91] Grew KN, Chiu WK. A dusty fluid model for predicting hydroxyl anion conductivity in alkaline anion exchange membranes. Journal of the electrochemical Society. 2010 Jan 4;157(3):B327.

[92] Zhang XM, Elkoun S, Ajji A, Huneault MA. Oriented structure and anisotropy properties of polymer blown films: HDPE, LLDPE and LDPE. Polymer. 2004 Jan $31 ; 45(1): 217-29$.

[93] Khuong KS, Jones WH, Pryor WA, Houk KN. The mechanism of the self-initiated thermal polymerization of styrene. Theoretical solution of a classic problem. Journal of the American Chemical Society. 2005 Feb 2;127(4):1265-77.

[94] Kim KJ, Lee SB, Han NW. Effects of the degree of crosslinking on properties of poly (vinyl alcohol) membranes. Polymer journal. 1993 Dec;25(12):1295-302.

[95] Dekel DR. Review of cell performance in anion exchange membrane fuel cells. Journal of Power Sources 2018, 375:158-69.

[96] Luo T, Abdu S, Wessling M. Selectivity of ion exchange membranes: A review. Journal of membrane science. 2018 Jun 1;555:429-54.

[97] Zeng QH, Liu QL, Broadwell I, Zhu AM, Xiong Y, Tu XP. Anion exchange membranes based on quaternized polystyrene-block-poly (ethylene-ran-butylene)-blockpolystyrene for direct methanol alkaline fuel cells. Journal of Membrane Science. 2010 Mar 1;349(1-2):237-43.

[98] Faraj M, Boccia M, Miller H, Martini F, Borsacchi S, Geppi M, Pucci A. New LDPE based anion-exchange membranes for alkaline solid polymeric electrolyte water electrolysis. international journal of hydrogen energy. 2012 Oct 1;37(20):14992-5002. 
[99] Gunatilake, S. K. "Methods of removing heavy metals from industrial wastewater." Methods 1, no. 1 (2015): 14.

[100] Jeevanantham S, Hosimin S, Vengatesan S, Sozhan G. Quaternized poly (styreneco-vinylbenzyl chloride) anion exchange membranes: role of different ammonium cations on structural, morphological, thermal and physio-chemical properties. New Journal of Chemistry. 2018;42(1):380-7.

[101] Barakat MA. New trends in removing heavy metals from industrial wastewater. Arabian Journal of Chemistry. 2011 Oct 31;4(4):361-77.

[102] Luo W, Wang X, Huang R, Fang P. Interface enhancement of glass fiber/unsaturated polyester resin composites with nano-silica treated using silane coupling agent. Wuhan University Journal of Natural Sciences. 2014 Feb 1;19(1):34-40.

[103] Jang J, Kim HS. Performance improvement of glass fiber-poly (phenylene sulfide) composite. Journal of applied polymer science. 1996 Jun 20;60(12):2297-306.

[104] Moghbeli, M. R., A. Khajeh, and M. Alikhani. "Nanosilica reinforced ion-exchange polyHIPE type membrane for removal of nickel ions: Preparation, characterization and adsorption studies." Chemical Engineering Journal 309 (2017): 552-562.

[105] Zhang, Kaizhou, Qiang Guo, Daohai Zhang, and Jianbing Guo. "Mechanical properties and morphology of polypropylene/polypropylene-g-maleic anhydride/long glass fiber composites." Journal of Macromolecular Science, Part B 54, no. 3 (2015): 286-294.

[106] Ku-Herrera JJ, Avilés F, Nistal A, Cauich-Rodríguez JV, Rubio F, Rubio J, Bartolo-Pérez P. Interactions between the glass fiber coating and oxidized carbon nanotubes. Applied Surface Science. 2015 Mar 1;330:383-92.

[107] Huang, Li, Hao Liu, and Chengzhong Wang. "Preparation and characterization of poly (styrene - co - divinylbenzene)/fiberglass cation - exchange composites." Journal of Applied Polymer Science 120, no. 2 (2011): 944-949.

[108] Somnuk, Usa, Nantaya Yanumet, John W. Ellis, Brian P. Grady, and Edgar A. O'Rear. "Adhesion improvement in glass fiber reinforced polyethylene composite via admicellar polymerization." Polymer composites 24, no. 1 (2003): 171-180.

[109] Saito, Kaori, Kyoichi Saito, Kazuyuki Sugita, Masao Tamada, and Takanobu Sugo. "Cation-exchange porous hollow-fiber membranes prepared by radiation-induced cografting of GMA and EDMA which improved pure water permeability and sodium ion adsorptivity." Industrial \& engineering chemistry research 41, no. 23 (2002): 5686-5691. 
[110] Křrivčík, J., D. Neděla, and R. Válek. "Ion-exchange membrane reinforcing." Desalination and Water Treatment 56, no. 12 (2015): 3214-3219.

[111] Annala, Minna, Sami Lipponen, Tanja Kallio, and Jukka Seppälä. "Proton conductive reinforced poly (ethylene - co - styrene) membranes." Journal of applied polymer science 124 , no. 2 (2012): 1511-1519.

[112] Bari, Pravin, Shital Lanjewar, Dharmesh Parshottam Hansora, and Satyendra Mishra. "Influence of the coupling agent and graphene oxide on the thermal and mechanical behavior of tea dust-polypropylene composites." Journal of Applied Polymer Science 133, no. 4 (2016).

[113] Bikiaris, D., P. Matzinos, A. Larena, V. Flaris, and C. Panayiotou. "Use of silane agents and poly (propylene - $\mathrm{g}$ - maleic anhydride) copolymer as adhesion promoters in glass fiber/polypropylene composites." Journal of Applied Polymer Science 81, no. 3 (2001): 701-709.

[114] Thomason, J. L. "The influence of fibre length and concentration on the properties of glass fibre reinforced polypropylene: 5. Injection moulded long and short fibre PP." Composites Part A: Applied Science and Manufacturing 33, no. 12 (2002): 1641-1652.

[115] Ibarra, Luis, and David Panos. "Dynamic properties of thermoplastic butadienestyrene (SBS) and oxidized short carbon fiber composite materials." Journal of Applied Polymer Science 67, no. 10 (1998): 1819-1826.

[116] Huang Y, Cheng T, Zhang X, Zhang W, Liu X. Novel composite proton exchange membrane with long-range proton transfer channels constructed by synergistic effect between acid and base functionalized graphene oxide. Polymer. 2018 Aug 1;149:305-15.

[117] Lin CW, Lu YS. Highly ordered graphene oxide paper laminated with a Nafion membrane for direct methanol fuel cells. Journal of Power Sources. 2013 Sep 1;237:18794.

[118] Długołęcki P, Ogonowski P, Metz SJ, Saakes M, Nijmeijer K, Wessling M. On the resistances of membrane, diffusion boundary layer and double layer in ion exchange membrane transport. Journal of Membrane Science. 2010 Mar 1;349(1-2):369-79.

[119] Choi BG, Hong J, Park YC, Jung DH, Hong WH, Hammond PT, Park H. Innovative polymer nanocomposite electrolytes: nanoscale manipulation of ion channels by functionalized graphenes. ACS nano. 2011 Jun 28;5(6):5167-74.

[120] Chen J, Li Y, Huang L, Li C, Shi G. High-yield preparation of graphene oxide from small graphite flakes via an improved Hummers method with a simple purification process. Carbon. 2015 Jan 1;81:826-34. 
[121] Aragaw BA, Su WN, Rick J, Hwang BJ. Highly efficient synthesis of reduced graphene oxide-Nafion nanocomposites with strong coupling for enhanced proton and electron conduction. RSC Advances. 2013;3(45):23212-21.

[122] Zarrin H, Higgins D, Jun Y, Chen Z, Fowler M. Functionalized graphene oxide nanocomposite membrane for low humidity and high temperature proton exchange membrane fuel cells. The Journal of Physical Chemistry C. 2011 Oct 27;115(42):2077481 .

[123] Kowsari E, Zare A, Ansari V. Phosphoric acid-doped ionic liquid-functionalized graphene oxide/sulfonated polyimide composites as proton exchange membrane. International Journal of Hydrogen Energy. 2015 Oct 26;40(40):13964-78.

[124] Jiang Z, Shi Y, Jiang ZJ, Tian X, Luo L, Chen W. High performance of a freestanding sulfonic acid functionalized holey graphene oxide paper as a proton conducting polymer electrolyte for air-breathing direct methanol fuel cells. Journal of Materials Chemistry A. 2014;2(18):6494-503.

[125] Liu S, Li D, Wang L, Yang H, Han X, Liu B. Ethylenediamine-functionalized graphene oxide incorporated acid-base ion exchange membranes for vanadium redox flow battery. Electrochimica Acta. 2017 Mar 10; 230:204-11.

[126] Shukla G, Pandey RP, Shahi VK. Temperature resistant phosphorylated graphene oxide-sulphonated polyimide composite cation exchange membrane for water desalination with improved performance. Journal of Membrane Science. 2016 Dec 15;520:972-82.

[127] Pandey TP, Maes AM, Sarode HN, Peters BD, Lavina S, Vezzu K, Yang Y, Poynton SD, Varcoe JR, Seifert S, Liberatore MW. Interplay between water uptake, ion interactions, and conductivity in an e-beam grafted poly (ethylene-co-tetrafluoroethylene) anion exchange membrane. Physical Chemistry Chemical Physics. 2015;17(6):4367-78.

[128] Hara M, Kimura T, Nakamura T, Shimada M, Ono H, Shimada S, Miyatake K, Uchida M, Inukai J, Watanabe M. Effect of surface ion conductivity of anion exchange membranes on fuel cell performance. Langmuir. 2016 Sep 20;32(37):9557-65.

[129] Müller F, Ferreira CA, Azambuja DS, Alemán C, Armelin E. Measuring the proton conductivity of ion-exchange membranes using electrochemical impedance spectroscopy and through-plane cell. The Journal of Physical Chemistry B. 2014 Jan 30;118(4):110212 .

[130] Siracusano S, Trocino S, Briguglio N, Baglio V, Aricò AS. Electrochemical impedance spectroscopy as a diagnostic tool in polymer electrolyte membrane electrolysis. Materials. 2018 Aug;11(8):1368. 
[131] Shukla G, Shahi VK. Sulfonated poly (ether ether ketone)/imidized graphene oxide composite cation exchange membrane with improved conductivity and stability for electrodialytic water desalination. Desalination. 2019 Feb 1; 451:200-8.

[132] Sharma PP, Kulshrestha V. Synthesis of highly stable and high water retentive functionalized biopolymer-graphene oxide modified cation exchange membranes. RSC advances. 2015;5(70):56498-506. 


\section{A Copyright documentation}

All images in this document are from Wikipedia. They are all public domain, or licensed for reuse under Creative Commons license 3.0. Please see below for full citation and attribution information.

Figure 1.1: " Preparation of the new polymer electrolyte membranes by irradiation grafting method" by Seyed Jamaleddin Peighambardoust. Downloaded from https://www.researchgate.net/figure/e-Preparation-of-the-new-polymer-electrolytemembranes-by-irradiation-grafting-method fig10 222897063. Accessed July 2020.

Figure 1.2: "Differences between a monomer, a conventional polymer and the polymer prepared by plasma polymerization" by Seyed Jamaleddin Peighambardoust. Downloaded from https://www.researchgate.net/figure/e-Differences-between-amonomer-a-conventional-polymer-and-the-polymer-prepared-by fig12 222897063. Accessed July 2020.

Figure 1.3: "The simple scheme of the hopping mechanism" by Seyed Jamaleddin Peighambardoust. Downloaded from https://www.researchgate.net/figure/e-The-simplescheme-of-the-hopping-mechanism-reprinted-with-permission-from-16 fig4 222897063. Accessed July 2020.

Figure 4.3: "The branching degree of LDPE, LLDPE and HDPE" by at the English language Wikipedia. Licensed under CC BY-SA 3.0 via Wikimedia Commonshttps://en.wikipedia.org/wiki/Polyethylene. Accessed July 2020. 